\title{
Investigating Custodial Symmetry Violation in the Georgi-Machacek Model
}

\author{
by \\ Ben Keeshan \\ A thesis submitted to the \\ Faculty of Graduate and Postdoctoral Affairs \\ in partial fulfillment of the requirements \\ for the degree of \\ Doctor of Philosophy in Physics \\ Department of Physics \\ Carleton University \\ Ottawa-Carleton Institute of Physics \\ Ottawa, Canada \\ September 1, 2021 \\ Copyright (C) 2021 Ben Keeshan
}




\section{Abstract}

The Georgi-Machacek (GM) model is the simplest benchmark model for higher isospin multiplet extensions of the Standard Model (SM) which allow for large contributions to electro-weak symmetry breaking (EWSB) from these 'exotic' multiplets. It is used to motivate and interpret Large Hadron Collider (LHC) searches for doubly charged scalars decaying to vector boson pairs. It preserves custodial symmetry (CS) at tree level; however, this CS has long been known to be violated at the one-loop level by hypercharge interactions. In this thesis, we study the effects of CS violation (CV). We assume that the CS GM model arises at a high scale as a result of an unspecified ultraviolet (UV) completion, and quantify the CV induced at the weak scale. We show that experimental data constrains the UV scale to lie below tens to hundreds of $\mathrm{TeV}$ over almost all of the parameter space. Subject to this constraint, we quantify the size of other CV effects at the weak scale and we find that these effects are small enough that they are unlikely to be probed by the LHC, but may be detectable at a future $e^{+} e^{-}$collider. We note that the upper bound on the UV scale is large enough that virtual effects from the UV completion will likely be undetectable at the LHC. This means that the GM model is a valid effective theory for LHC physics.

We also propose a new "low- $m_{5}$ " benchmark for the tree level GM model, defined for $m_{5} \in(50,550) \mathrm{GeV}$, and characterize its properties. This benchmark plane is designed to facilitate the extension of current collider searches for doubly charged scalars to an unexplored region of parameter space which was not previously covered by a benchmark plane. The doubly charged scalars are part of a degenerate fermio-

phobic custodial fiveplet with states $H_{5}^{ \pm \pm}, H_{5}^{ \pm}$, and $H_{5}^{0}$ and the input parameter $m_{5}$ is their common mass. We apply all existing experimental constraints and summarize the phenomenology of the surviving parameter space. We show that there is a largely 
unprobed region of parameter space from $120 \mathrm{GeV}<m_{5}<200 \mathrm{GeV}$ where light doubly-charged scalars could exist. 


\section{Acknowledgments}

This thesis is the culmination of academic pursuits stretching out over many years and as such there are a great deal many people whom I owe thanks. Even if I were to list all the friends, family, professors, students, teaching assistants, colleagues, and casual acquaintances who at various times have given me their time, their support, or served as a source of motivation or inspiration, I am afraid that I have always found words a poor medium for the adequate expression of gratitude and if I were to attempt to individually thank everyone who deserved it, I am sure that I would run on and on to little purpose. For all who have had a positive impact on this thesis, especially those not specifically mentioned below, I hope it will suffice to merely state that you have my most sincere thanks; at the very least as much thanks as you think you deserve and in some cases undoubtedly far more than you could ever know.

With the above sentiment in mind, I will only specifically acknowledge the contributions of a select few of many deserving people. Firstly, I am extremely grateful to my supervisor Heather Logan who has made this process as enjoyable and rewarding as one could possibly hope. I want to thank my collaborators: Terry Pilkington, Ameen Ismail, Yongcheng Wu, Yue Zhang, and Carlos Henrique de Lima. Working with you all was a genuine pleasure. I also want to express my gratitude to my thesis committee for taking the time to help me improve both my research and my thesis. I am also grateful to the members of the Carleton Theory Group for many stimulating discussions, fun diversions, and reference letters as the case may be. Speaking of reference letters, I also want to thank Weihua Zhang who wrote a great many on my behalf. I also want to extend thanks to Shinya Kanemura and the department of physics at Osaka University for their hospitality.

This thesis would have been much more difficult without the financial support of 
many institutions. I want to thank everyone who has funded my research throughout my academic career especially Carleton University, the National Science and Engineering Research Council of Canada, MITACS, and the government of Ontario.

Finally I want to thank my family: my parents, David and Valerie; and my sisters, Sarah, Melissa, Alexa, and Bethany for their love and support in this as in all things. It has been greatly appreciated. 


\section{Statement of Originality}

Chapter 2 of this thesis is primarily a literature review of background material and methods. Chapters 3 and 4 contain the new results of the thesis and are primarily based on the author's publications Refs. [1] and [2] respectively. They also include results which were produced by collaborators and work based on the author's honours project as described below.

- The material in the sections before Sec. 4.3.6 contains background material for the Georgi-Machacek model.

- Sec. 4.3.6 contains the new results from Ref. [1]

The benchmark presented in Sec. 3.3 was created collaboratively by both the author and Heather Logan.

The results of Sec. 3.3.1- 3.3.2.2 are the independent work of the author using pre-existing publicly available code as described in the text. The only exception is the $H_{5}$ production cross sections given in Fig. 3.7 which were calculated by collaborator Yongcheng Wu.

- The most general gauge invariant model given in Sec. 4.1, its relationship to the GM model and the mass-mixing matrices given in Sec. 4.2 are based on work done for the author's BSc's honours project.

- All the results of Sec. 4.3 and the numerical method described therein are the independent work of the author.

- The RGEs given in App. A were calculated by my collaborator Terry Pilkington

- The couplings given in App. B were derived independently by the author 
With the above caveats in mind, the research presented in this thesis is entirely new and original (to the best of our knowledge) and has not been submitted as the final project of another degree. 


\section{Contents}

1 Introduction 1

2 The Standard Model $\quad 7$

2.1 The Particles of the $\mathrm{SM} \ldots \ldots \ldots . \ldots . \ldots$

2.2 The Theoretical Framework of the SM . . . . . . . . . . . 13

2.2.1 Quantum Field Theory . . . . . . . . . . . . . 14

2.2.2 Chirality and the $\mathrm{SM} \ldots \ldots . \ldots 20$

2.2.3 Gauge Symmetry . . . . . . . . . . . . . . . . . . . 21

2.3 The Higgs mechanism . . . . . . . . . . . . . . . . . . . 25

2.3.1 Custodial Symmetry . . . . . . . . . . . . . 38

2.3.2 Low Energy Observables of EWSB . . . . . . . . . . . . 41

2.4 Beyond Tree-Level: Renormalized Perturbation Theory . . . . . . . . 44

2.5 Beyond Tree-Level: Renormalization Group Equations . . . . . . . . 54

2.6 Beyond the $\mathrm{SM} \ldots \ldots \ldots$. . . . . . . . . . . . . . 62

3 The Georgi-Machacek Model $\quad 69$

3.0.1 Higher Isospin Extensions of the SM . . . . . . . . . . . 72

3.1 The Model . . . . . . . . . . . . . . . . . . . 74

3.2 Phenomenology ........................ 80 
3.3 Low- $m_{5}$ benchmark . . . . . . . . . . . . . . . . . . 84

3.3.1 Populated range of the $m_{5}-s_{H}$ plane and existing constraints . 86

3.3.2 Phenomenology of the low- $m_{5}$ benchmark . . . . . . . 93

$3.3 .2 .1 \quad$ Couplings of $h \ldots \ldots \ldots \ldots$

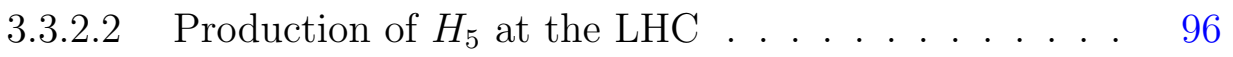

3.3.2.3 Decays of $H_{5} \ldots \ldots \ldots \ldots$

3.3 .3 Summary . . . . . . . . . . . . . . . . . . 102

4 Custodial Violation in the GM Model 105

4.1 Custodial violation and the most general gauge-invariant scalar potential109

4.2 Physical masses and mixing in the custodial symmetry violating theory 114

4.3 Numerical results . . . . . . . . . . . . . . . . . . . . . . . . . 122

4.3.1 Calculational procedure . . . . . . . . . . . . . . . . 122

4.3.2 Constraints on the cutoff scale from perturbativity and the $\rho$ parameter . . . . . . . . . . . . . . . . 128

4.3 .3 Custodial violation in couplings . . . . . . . . . . . 138

4.3.4 Custodial-violating mass splittings _. . . . . . . . . . . 149

4.3.5 Direct search constraints . . . . . . . . . . . . . . 159

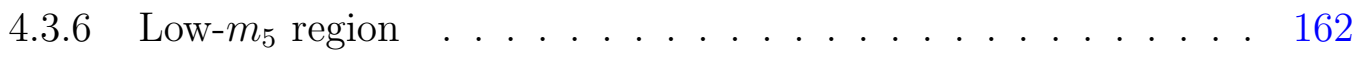

4.4 Summary . . . . . . . . . . . . . . . . . . 170

$\begin{array}{lll}5 & \text { Conclusion } & 174\end{array}$

A Renormalization group equations for Lagrangian parameters $\quad 179$

B Scalar couplings of the custodial violating states 185 


\section{List of Tables}

2.1 List of all known fundamental particles and some of their properties [4]. All the masses are given in natural units, where $\hbar=c=1$ and the electric charge is given in units of $e$. The anti-neutrinos are distinguished from neutrinos based on their chirality (right-handed versus left handed). It is unknown whether neutrinos and anti-neutrinos are actually distinct or if they are the same particle (i.e. Dirac or Majorana particles). See for e.g. Ref. [30]. The quarks each carry one of the 3 possible colour charges (red, blue and green) so each type of quark has 3 distinct particles which are otherwise indistinguishable. The gluon carries a colour and anti-colour charge with 8 possible charge assignments, meaning there are 8 gluons. . . . . . . . . . .

2.2 Field content of the SM and their representations under the gauge group. The gauge forces which are described in the SM are the strong force $\left(S U(3)_{C}\right)$, weak isospin $\left(S U(2)_{L}\right)$, and hypercharge $\left(U(1)_{Y}\right)$. In

this thesis we use the convention $Q=T^{3}+\frac{Y}{2}$. At low energies, the gauge symmetry of the SM is broken and the gauge group breaks down to $S U(3)_{C} \times S U(2)_{L} \times U(1)_{Y} \rightarrow S U(3)_{C} \times U(1)_{E M} \ldots \ldots . . .$. 
3.1 Input parameters for the H5plane benchmark scenario [21] in the custodialsymmetric GM model. . . . . . . . . . . . . . . . . . 83

3.2 Specification of the low- $m_{5}$ benchmark for the Georgi-Machacek model. This set of input parameters can be accessed in GMCALC [76] by using $\operatorname{INPUTSET}=6 \ldots \ldots \ldots \ldots$. . . . . . . . . . . 86

4.1 Input parameters for the general scan in the custodial-symmetric GM model. . . . . . . . . . . . . . . . . . . . . 124

4.2 Input parameters for the dedicated low- $m_{5}$ scan in the custodial-symmetric GM model. . . . . . . . . . . . . . . . . . . . . . . 124 


\section{List of Figures}

2.1 Example of tree-level Feynman diagrams. Different particles are conventionally represented by different lines. Straight lines represent fermions (with the direction of the arrow differentiating fermion from antifermions), wavey lines represent electro-weak gauge bosons, and curly lines represent gluons. Left: Example vertices from which Feynman diagrams are built. Each vertex represents a rule which can be read from the corresponding interaction term in the Lagrangian. Right: Examples of tree-level Feynman diagrams describing $2 \rightarrow 2$ fermion scattering. . . . . . . . . . . . . . . . . 18

2.2 The four possible forms of the potential as a function of $|\phi|$. These correspond to positive $-\mu^{2}$ and $\lambda$ (top left), negative $-\mu^{2}$ and positive $\lambda$ (top right), negative $-\mu^{2}$ and $\lambda$ (bottom left), and positive $-\mu^{2}$ and negative $\lambda$ (bottom right) . . . . . . . . . . . . . . 28

2.3 The diagram which mixes Goldstone bosons and broken gauge bosons 33

2.4 The interactions between the massive electro-weak gauge bosons and the Higgs boson generated by EWSB. . . . . . . . . . . . . . . . . . . 37

2.5 An example of an effective 4 -Fermi vertex . . . . . . . . . . . . 41

2.6 The 7 possible superficially divergent diagrams of pseudo-Yukawa theory. 48 
2.7 The diagrams which contribute to the 1-loop renormalization of the Yukawa coupling g. . . . . . . . . . . . . . . .

3.1 Searches for the charged fiveplet states of the GM model at the LHC. Parameter space above the solid black curve is excluded. Top left: CMS search for $H_{5}^{+}$[15] (Fig. 3-b). Top right: ATLAS search for $H_{5}^{+}$[17] (Fig. 7-b). Bottom left: CMS search for $H_{5}^{++}$[16] (Fig. 3-b). An update to this search [77] was published after the finalization of our analysis. See text for a summary of the new limit. Bottom right: ATLAS search for $H_{5}^{++} \rightarrow W W \rightarrow e \nu \mu \nu$ [19] (Fig. 10-b). These plots are reproduced unaltered from the cited references under CC-BY-3.0 [C 2015 CERN] . . . . . . . . . . . . . . . .

3.2 The region of $m_{5}$ versus $s_{H}$ that is populated by the low- $m_{5}$ benchmark (black) compared to a general scan over the full parameter space (red), imposing the same theoretical and indirect experimental constraints $\left(S\right.$ parameter and $\left.B_{s} \rightarrow \mu \mu\right)$. Because our general scan is less efficient for small $m_{5}$ values, we include a dedicated general scan restricted to $m_{5}<200 \mathrm{GeV}$ overlaid with a scan for $m_{5}<600 \mathrm{GeV}$ in order to generate a sufficient number of points below $200 \mathrm{GeV}$. . . . . . . . 
3.3 The excluded (colored) and allowed (black) points in the scan of the low-mass benchmark shown in Fig. 3.2. The black points are unconstrained, the dark blue points are excluded by the CMS Run 2 direct search for likesign dileptons [16], the orange points are excluded by the ATLAS Run 2 direct search for $H_{5}^{ \pm \pm}$[20] (this exclusion is extended from $m_{5}=220 \mathrm{GeV}$ to $m_{5}=350 \mathrm{GeV}$ when one considers the updated search [84]), the light blue points are excluded by the recast of the ATLAS Run 1 diphoton resonance search [86], the yellow points are excluded by the recast of the ATLAS Run 1 constraint on anomalous like-sign dimuon production [84], the teal points are excluded by the recast of the ATLAS Run $1 \mathrm{VBF} \rightarrow W^{+} W^{+} \rightarrow$ like-sign dileptons cross section measurement [82], and the red points are excluded by searches implemented in HiggsBounds 5.3 .2 [79]. . . . . . . . . . . . . 89

3.4 The regions of the $m_{5}-s_{H}$ plane populated by the low- $m_{5}$ benchmark (black) and by a general scan (red) after all experimental constraints have been applied. . . . . . . . . . . . . . . . .

3.5 Coupling modification factor $\kappa_{f}^{h}$ for the SM-like Higgs $h$ coupling to fermion pairs in the low- $m_{5}$ benchmark, as a function of $m_{5}$ (top) and $s_{H}$ (bottom). The values range between 1 and $1.19 . \ldots . . . .$.

3.6 Coupling modification factor $\kappa_{V}^{h}$ for the SM-like Higgs $h$ coupling to vector boson pairs in the low- $m_{5}$ benchmark, as a function of $m_{5}$ (top) and $s_{H}$ (bottom). The values range between 1 and $1.08 \ldots \ldots . .$. 
3.7 Production cross sections for Drell-Yan production of pairs of $H_{5}$ states (left, independent of $s_{H}$ ) and VBF production of single $H_{5}$ states (right, for $s_{H}=0.1$ ) at the $13 \mathrm{TeV}$ LHC, computed at NLO in QCD using MadGraph5_aMC@NLO. This figure was produced by my collaborator Yongcheng $\mathrm{Wu}$ and is used with permisson. . . . . . . . . . .

3.8 Contours of cross sections for the resonant processes $p p \rightarrow H \rightarrow$ $H_{5}^{++} H_{5}^{--}$(equal to $p p \rightarrow H \rightarrow H_{5}^{+} H_{5}^{-}$) (top) and $p p \rightarrow H \rightarrow H_{5}^{0} H_{5}^{0}$ (bottom) in the low- $m_{5}$ benchmark for the $13 \mathrm{TeV}$ LHC. The shaded region has $m_{H}>2 m_{5}$ but is excluded by experimental searches. The region with contours above the dotted black line has $m_{H}>2 m_{5}$ and is still allowed. For $p p \rightarrow H \rightarrow H_{5}^{++} H_{5}^{--}$and $p p \rightarrow H \rightarrow H_{5}^{+} H_{5}^{-}$, the minimum allowed cross section is $0.230 \mathrm{fb}$ and the maximum allowed is $21.5 \mathrm{fb}$. For $p p \rightarrow H \rightarrow H_{5}^{0} H_{5}^{0}$ the minimum allowed cross-section is $0.115 \mathrm{fb}$ and the maximum allowed is $10.7 \mathrm{fb}$. . . . . . . . . . . 101

3.9 Contours of branching ratios of the loop-induced decays of $H_{5}^{0} \rightarrow \gamma \gamma$ (top) and $H_{5}^{+} \rightarrow W^{+} \gamma$ (bottom) in the low- $m_{5}$ benchmark. The region above and to the left of the dotted line is excluded by direct searches. The shaded gray area in the top panel is excluded by diphoton resonance searches applied to $H_{5}^{0} \rightarrow \gamma \gamma$. For $m_{5}<200 \mathrm{GeV}$, the values of $B R\left(H_{5}^{0} \rightarrow \gamma \gamma\right)$ in the low- $m_{5}$ benchmark range between $1.26 \times 10^{-6}$ and 1.00, surpassing $99 \%$ for $m_{5} \lesssim 80 \mathrm{GeV}$. The values of $B R\left(H_{5}^{+} \rightarrow W^{+} \gamma\right)$ range between zero and 0.887 with the maximum at $m_{5} \simeq 94 \mathrm{GeV}$. 
4.1 Constraints on the custodial-symmetric cutoff scale due to perturbativity of the model in the H5plane benchmark. Top: the scale of the Landau pole, defined as the scale at which any of the $\lambda_{i}$ in the custodial-symmetric theory becomes larger than $10^{3}$. This scale varies between $2.5 \mathrm{TeV}$ and $2594.2 \mathrm{TeV}$ over the benchmark considered. Bottom: the highest scale at which the perturbative unitarity constraints of Eq. (3.20) in the custodial-symmetric theory remain satisfied. This scale varies between $346.8 \mathrm{GeV}$ and $291.1 \mathrm{TeV}$ over the benchmark

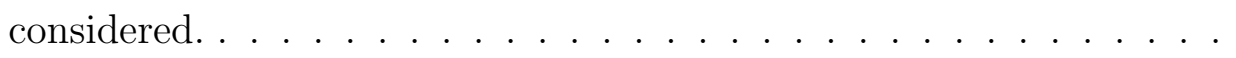

4.2 Values of and constraints due to the $\rho$ parameter in the H5plane benchmark. Top: the highest scale at which the perturbative unitarity constraints of Eq. (3.20) in the custodial-symmetric theory remain satisfied as in the right panel of Fig. 4.1 (solid lines), showing also the highest allowed custodial-symmetric scale after requiring that the $\rho$ parameter remain within $\pm 2 \sigma$ of its experimental value [Eq. (4.60)] in the custodial-violating weak-scale theory (dashed lines). The range of scales allowed after imposing the constraint on $\rho$ remains the same as in Fig. 4.1. Bottom: the value of $\rho$ in the weak-scale custodial-violating theory when the custodial-symmetric scale is taken as large as possible subject to perturbative unitarity at the high scale and the experimental limits on $\rho$. The values of $\rho$ range between the $\pm 2 \sigma$ limits of 0.99991

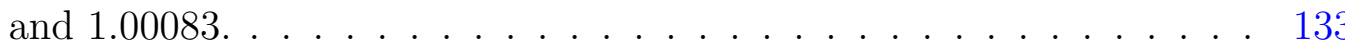


4.3 The highest allowed custodial-symmetric cutoff scale due to perturbative unitarity of the quartic couplings in a general scan (red) and in the H5plane benchmark (black), as a function of $m_{5}$ (top) and $s_{H}$ (bottom). The highest allowed cutoff scale in the general scan ranges between $207 \mathrm{GeV}$ and $1.6 \times 10^{11} \mathrm{GeV}$, though almost all points lie below $\sim 2 \times 10^{9} \mathrm{GeV}$. (The point with the highest cutoff scale is at the upper edge of the plots at $m_{5}=681 \mathrm{GeV}$ and $\left.s_{H} \simeq 0.12\right) \ldots \ldots$

4.4 The highest allowed custodial-symmetric cutoff scale imposing perturbative unitarity and the requirement that the weak-scale $\rho$ parameter lie within $\pm 2 \sigma$ of its measured value. Red points are for a general scan and black are for the H5plane benchmark, as a function of $m_{5}$ (top) and $s_{H}$ (bottom). The highest allowed cutoff scale in the general scan ranges between $205 \mathrm{GeV}$ and $1.8 \times 10^{9} \mathrm{GeV}$, though almost all the points lie below $\sim 2 \times 10^{6} \mathrm{GeV}$. (The point with the highest cutoff scale is at the upper edge of the plots at $m_{5}=681 \mathrm{GeV}$ and $\left.s_{H} \simeq 0.12\right) .136$

4.5 The weak-scale $\rho$ parameter evaluated with the cutoff scale at its maximum allowed value in a general scan (red) and in the H5plane benchmark (black), as a function of $m_{5}$ (top) and $s_{H}$ (bottom). Both the general scan and the H5plane benchmark populate the entire $\pm 2 \sigma$ allowed region of $\rho \in(0.99991,1.00083)$. . . . . . . . . . 137

4.6 Contours of $\delta \lambda_{W Z}^{\tilde{h}} \equiv \lambda_{W Z}^{\tilde{h}}-1$ in the H5plane benchmark, taking the scale of the custodial-symmetric theory to be as large as possible subject to perturbative unitarity and the $\rho$ parameter constraint. $\delta \lambda_{W Z}^{\tilde{h}}$ varies between $-5.1 \times 10^{-3}$ and $1.4 \times 10^{-3}$. . . . . . . . . . 139 
$4.7 \lambda_{W Z}^{\tilde{h}} \equiv \kappa_{W}^{\tilde{h}} / \kappa_{Z}^{\tilde{h}}$ evaluated with the maximum allowed cutoff scale for a general parameter scan (red) and in the H5plane benchmark (black), as a function of $m_{5}$ (top) and $s_{H}$ (bottom). The minimum value in the general scan is 0.99934 and the maximum value is 1.00197 . . . . . .

4.8 The coupling of $\tilde{H}_{5}^{0}$ to fermions and the resulting fermionic branching ratio in the H5plane benchmark, taking the scale of the custodialsymmetric theory to be as large as possible subject to perturbative unitarity and the $\rho$ parameter constraint. Top: contours of $\kappa_{f}^{\tilde{H}_{5}^{0}}$ [defined above Eq. (4.54)]. The allowed values range between $-4.0 \times 10^{-2}$ and $2.0 \times 10^{-3}$. Bottom: contours of the branching ratio of $\tilde{H}_{5}^{0}$ to fermions. We compute only the partial width to the heaviest kinematically accessible pair of fermions; i.e., to $t \bar{t}$ for $m_{\tilde{H}_{5}^{0}}>2 m_{t}$ and $b \bar{b}$ otherwise. The branching ratio of $\tilde{H}_{5}^{0}$ to fermions ranges from $3.5 \times 10^{-11}$ to $4.8 \times 10^{-3} \ldots \ldots \ldots \ldots \ldots \ldots \ldots$

4.9 The coupling $\kappa_{f}^{\tilde{H}_{5}^{0}}$ of $\tilde{H}_{5}^{0}$ to fermions evaluated with the maximum allowed cutoff scale in a general parameter scan (red) and in the H5plane benchmark (black), as a function of $m_{5}$ (top) and $s_{H}$ (bottom). In the general scan the coupling ranges between -0.50 and +0.50 (for rare points at large $s_{H}$ between 0.5 and 0.6 ). This coupling is zero in the custodial-symmetric model. . . . . . . . . . . . . . . . . . . 143

4.10 Branching ratio of $\tilde{H}_{5}^{0} \rightarrow f \bar{f}$ evaluated with the maximum allowed cutoff scale in a general parameter scan (red) and in the H5plane benchmark (black), as a function of the mass difference $m_{\tilde{H}_{5}^{0}}-m_{\tilde{H}}$ showing the resonant mixing effect (top) and $m_{5}$ (bottom). The maximum branching ratio to fermions in the general scan is $19 \%$. . . . . . . 144 
4.11 The coupling of $\tilde{H}_{5}^{+}$to fermions and the resulting fermionic branching ratio in the H5plane benchmark, taking the scale of the custodialsymmetric theory to be as large as possible subject to perturbative unitarity and the $\rho$ parameter constraint. Top: contours of $\kappa_{f}^{\tilde{H}_{5}^{+}}$[defined in Eq. (4.36)]. The allowed values range between $1.0 \times 10^{-4}$ and $5.2 \times 10^{-2}$. Bottom: contours of the branching ratio of $\tilde{H}_{5}^{+}$to fermions, including only the decay to $t \bar{b}$. This branching ratio ranges from $2.0 \times 10^{-8}$ to $1.2 \times 10^{-2} \ldots \ldots \ldots \ldots$

4.12 The coupling $\kappa_{f}^{\tilde{H}_{5}^{+}}$of $\tilde{H}_{5}^{+}$to fermions evaluated with the maximum allowed cutoff scale in a general parameter scan (red) and in the H5plane benchmark (black), as a function of $m_{5}$ (top) and $s_{H}$ (bottom). In the general scan the coupling ranges between -0.22 and +0.29 . . . . . . 147

4.13 Branching ratio of $\tilde{H}_{5}^{+} \rightarrow t \bar{b}$ evaluated with the maximum allowed cutoff scale in a general parameter scan (red) and in the H5plane benchmark (black), as a function of the mass difference $m_{\tilde{H}_{5}^{+}}-m_{\tilde{H}_{3}^{+}}$showing the resonant mixing effect (top) and $m_{5}$ (bottom). The maximum branching ratio to fermions in the general scan is $29 \%$. . . . . . . . 148 
4.14 The mass splittings within the custodial triplet in the H5plane benchmark, taking the scale of the custodial-symmetric theory to be as large as possible subject to perturbative unitarity and the $\rho$ parameter constraint. Top: $m_{\tilde{H}_{3}^{+}}-m_{\tilde{H}_{3}^{0}}$. This quantity is negative because $\tilde{H}_{3}^{+}$is lighter than $\tilde{H}_{3}^{0}$. The mass splitting ranges between zero and $5.3 \mathrm{GeV}$. Bottom: $m_{\tilde{H}_{3}^{0}}-m_{3}$, where $m_{3}$ is the weak-scale custodial-symmetric input value of the custodial triplet mass. $m_{\tilde{H}_{3}^{0}}$ and $m_{\tilde{H}_{3}^{+}}$are both larger than $m_{3}$ over the entire benchmark. In our numerical scan, the difference between $m_{\tilde{H}_{3}^{0}}$ and $m_{3}$ ranges between $4 \mathrm{MeV}$ and $9.1 \mathrm{GeV}$.

4.15 Mass splitting $m_{\tilde{H}_{3}^{+}}-m_{\tilde{H}_{3}^{0}}$ evaluated with the maximum allowed cutoff scale in a general parameter scan (red) and in the H5plane benchmark (black), as a function of $m_{5}$ (top) and $s_{H}$ (bottom). This quantity is negative because $\tilde{H}_{3}^{+}$is lighter than $\tilde{H}_{3}^{0}$. The mass splitting in the general scan ranges between $+0.25 \mathrm{GeV}$ and $-23 \mathrm{GeV}$. . . . . . . . 152

4.16 The mass splittings within the custodial fiveplet in the H5plane benchmark, taking the scale of the custodial-symmetric theory to be as large as possible subject to perturbative unitarity and the $\rho$ parameter constraint. Top left: $m_{\tilde{H}_{5}^{++}}-m_{\tilde{H}_{5}^{0}}$. This mass splitting ranges between 4.0 MeV and $7.2 \mathrm{GeV}$. Top right: $m_{\tilde{H}_{5}^{+}}-m_{\tilde{H}_{5}^{0}}$. This mass splitting ranges between $6.0 \mathrm{MeV}$ and $1.8 \mathrm{GeV}$. Bottom left: $m_{\tilde{H}_{5}^{0}}-m_{5}$, where $m_{5}$ is the weak-scale custodial-symmetric input value of the custodial fiveplet mass. This mass difference ranges between $-1.5 \mathrm{GeV}$ and $2.3 \mathrm{GeV}$. Bottom right: $m_{\tilde{H}_{5}^{++}}-m_{5} \cdot m_{\tilde{H}_{5}^{++}}$is always larger than $m_{5}$, with the difference ranging between $7.0 \mathrm{MeV}$ and $9.0 \mathrm{GeV}$. . . . . . 153 
4.17 Mass splittings $m_{\tilde{H}_{5}^{++}}-m_{\tilde{H}_{5}^{0}}$ (top left), $m_{\tilde{H}_{5}^{+}}-m_{\tilde{H}_{5}^{0}}$ (top right), and $m_{\tilde{H}_{5}^{++}}-m_{\tilde{H}_{5}^{+}}$(bottom) as a function of $m_{5}$, evaluated with the maximum allowed cutoff scale in a general parameter scan (red) and in the H5plane benchmark (black). In the general scan $m_{\tilde{H}_{5}^{++}}-m_{\tilde{H}_{5}^{0}}$ ranges between zero and $34.5 \mathrm{GeV}, m_{\tilde{H}_{5}^{+}}-m_{\tilde{H}_{5}^{0}}$ ranges between $-1.45 \mathrm{GeV}$ and $22.3 \mathrm{GeV}$, and $m_{\tilde{H}_{5}^{++}}-m_{\tilde{H}_{5}^{+}}$ranges between zero and $34.4 \mathrm{GeV}$.

4.18 Deviation $m_{\tilde{H}}-m_{H}$ of the physical $\tilde{H}$ mass from the mass of the heavier custodial singlet $H$ in the weak-scale custodial-symmetric theory, computed in the H5plane benchmark taking the scale of the custodialsymmetric theory to be as large as possible subject to perturbative unitarity and the $\rho$ parameter constraint. This mass difference ranges between $-0.059 \mathrm{GeV}$ and $+5.6 \mathrm{GeV}$. . . . . . . . . . . . . 157

4.19 The fractional change in $\tilde{v}_{\chi}$ relative to the weak-scale custodial-symmetric input $v_{\chi}$, defined as $\frac{\tilde{v}_{\chi}}{v_{\chi}}-1$, in the H5plane benchmark taking the scale of the custodial-symmetric theory to be as large as possible subject to perturbative unitarity and the $\rho$ parameter constraint. The fractional change is always negative and its absolute value reaches a maximum of $1.0 \% \ldots \ldots \ldots \ldots \ldots$ 
4.20 The fractional change in $\tilde{v}_{\chi}$ relative to the weak-scale custodial-symmetric input $v_{\chi}$, defined as $\frac{\tilde{v}_{\chi}}{v_{\chi}}-1$, as a function of $m_{5}$ (top) and $s_{H}$ (bottom), evaluated with the maximum allowed cutoff scale in a general parameter scan (blue and red points) and in the H5plane benchmark (black points). The fractional change can be positive (blue points) or negative (red points) in the general scan, but is always negative in the H5plane benchmark (black points). The fractional change reaches maxima and minima of 0.55 and -1.66 , respectively, at very low $s_{H}$, but for $s_{H}>0.1$ its absolute value reaches at most $0.0114 \ldots$. . . . . . . . . . . . 161

4.21 Maximum value of the custodial-symmetric cutoff scale subject to perturbative unitarity and the experimental constraint on the $\rho$ parameter in a general scan of the low- $m_{5}$ region, as a function of $m_{5}$ (top) and $s_{H}$ (bottom). The maximum cutoff scale ranges from $35 \mathrm{GeV}$ (for very

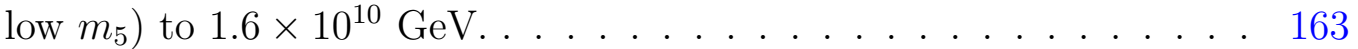

4.22 Value of the $\rho$ parameter in a general scan of the low- $m_{5}$ region as a function of $m_{5}$ (top) and $s_{H}$ (bottom), taking the scale of the custodialsymmetric theory to be as large as possible subject to perturbative unitarity and the $\rho$ parameter constraint. . . . . . . . . .

$4.23 \lambda_{W Z}^{\tilde{h}}$ in a general scan of the low- $m_{5}$ region as a function of $m_{5}$, taking the scale of the custodial-symmetric theory to be as large as possible subject to perturbative unitarity and the $\rho$ parameter constraint. The bottom panel is a zoom of the $y$-axis. In the resonant mixing region $m_{5} \simeq 125 \mathrm{GeV}$ we find values between 0.43 and 1.76 , while away from this region $\lambda_{W Z}^{\tilde{h}}$ can deviate from one by as much as $1-2 \%$. . . . . 165 
4.24 Custodial-symmetry-violation-induced couplings of $\tilde{H}_{5}^{+}$to fermions in a general scan of the low- $m_{5}$ region, taking the scale of the custodialsymmetric theory to be as large as possible subject to perturbative unitarity and the $\rho$ parameter constraint. Top: $\kappa_{f}^{\tilde{H}_{5}^{+}}$as a function of $m_{5}$. The minimum and maximum values are -0.29 and 0.34 respectively. Bottom: decay branching ratios of $\tilde{H}_{5}^{+}$to $W Z$ (red), $W \gamma$ (green), and $f \bar{f}$ (black) as a function of $m_{5}$. Decays to fermions are computed including only the dominant modes: $t b$ above the $t b$ threshold and $c s$ and $c d$ below. The calculation of $H_{5}^{+} \rightarrow W \gamma$ assumes an on-shell final-state $W$, so we plot the branching ratios only between 80 and $200 \mathrm{GeV}$

4.25 Custodial-symmetry-violation-induced couplings of $\tilde{H}_{5}^{0}$ to fermions in a general scan of the low- $m_{5}$ region, taking the scale of the custodialsymmetric theory to be as large as possible subject to perturbative unitarity and the $\rho$ parameter constraint. Top: $\kappa_{f}^{\tilde{H}_{5}^{0}}$ as a function of $m_{5}$. The minimum and maximum values are -0.46 and 0.71 respectively. Bottom: decay branching ratios of $\tilde{H}_{5}^{0}$ to $W W / Z Z$ (blue), $\gamma \gamma$ (red), $f \bar{f}$ (black), $Z \gamma$ (orange), and custodial-violating decays to pairs of other scalars (green - a few points in the upper right of the plot). . . . . . . 168 
4.26 Mass splittings among the members of the custodial fiveplet and triplet in a general scan of the low- $m_{5}$ region, taking the scale of the custodialsymmetric theory to be as large as possible subject to perturbative unitarity and the $\rho$ parameter constraint. For the fiveplet we show $m_{\tilde{H}_{5}^{++}}-m_{\tilde{H}_{5}^{0}}($ top left, ranging between $-0.08 \mathrm{GeV}$ and $10.6 \mathrm{GeV})$, $m_{\tilde{H}_{5}^{+}}-m_{\tilde{H}_{5}^{0}}$ (top right, ranging between $-0.33 \mathrm{GeV}$ and $2.51 \mathrm{GeV}$ ), and $m_{\tilde{H}_{5}^{++}}-m_{\tilde{H}_{5}^{+}}($bottom left, ranging between $-0.06 \mathrm{GeV}$ and $8.44 \mathrm{GeV})$, and for the triplet we show $m_{\tilde{H}_{3}^{+}}-m_{\tilde{H}_{3}^{0}}$ (bottom right, ranging between

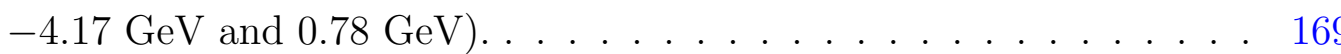

4.27 Fractional deviation of $\tilde{v}_{\chi}$ relative to the weak-scale custodial-symmetric input $v_{\chi}$, defined as $\frac{\tilde{v}_{\chi}}{v_{\chi}}-1$, as a function of $m_{5}$ (top) and $s_{H}$ (bottom) in a general scan of the low- $m_{5}$ region, taking the scale of the custodial-symmetric theory to be as large as possible subject to perturbative unitarity and the $\rho$ parameter constraint. Positive deviations are shown in black and negative in red so that both can be plotted on a $\log$ scale. The fractional deviation ranges between -1.66 and 0.55 , with these large deviations appearing mainly at very small $s_{H} \ldots \ldots$. . 171 


\section{Chapter 1}

\section{Introduction}

The Standard Model (SM) is the theory which describes the properties and interactions of all known fundamental particles. Its phenomenological successes have been tremendous; its predictions agree with experiment to an extremely high precision and it has successfully predicted the discovery of many new particles. With the discovery of a Higgs boson particle consistent with the SM almost a decade ago, the experimental verification of the SM was completed. However, for all its successes there are strong indications that the SM is incomplete as there are numerous experimental observations (for e.g. dark matter and massive neutrinos) that cannot be explained by the SM. The physics which explains these observations is often referred to as Beyond the SM (BSM) physics and its discovery is the primary focus of current research in particle physics. The discovery of a Higgs boson was of especial importance to the $\mathrm{SM}$ as it experimentally probes one of the key mechanisms of the SM which is responsible for the mass of the fundamental SM particles. The dynamics of this mechanism, known as electro-weak symmetry breaking (EWSB), can have a significant effect on the properties of SM particles and these dynamics tend to play a role in explanations

of BSM physics. It is thus very important that the possible dynamics of EWSB are 
studied and that the phenomenology of these different possibilities is characterized. The implementation of the dynamics of EWSB in the SM is through a single complex scalar field transforming as a doublet under the weak $\mathrm{SU}(2)_{L}$ gauge symmetry; in BSM scenarios the dynamics can be a more complex process.

The gauge symmetries of the massive SM fermions require at least one scalar $\mathrm{SU}(2)_{L}$ doublet, but the masses of the $W$ and $Z$ bosons can in principle also receive contributions from scalars in larger representations of $\mathrm{SU}(2)_{L}$ ("exotic" scalars). This possibility allows for 'exotic' phenomenological signatures which include new doublycharged Higgs bosons and unique modifications to the predicted properties of the discovered SM-like Higgs boson. These 'exotic' possibilities are severely constrained by the low-energy measurements of the $\rho$ parameter [3], defined as the ratio of the strengths of the neutral and charged weak currents in the low-energy limit and measured to very high precision via the global electroweak fit [4]. In general, the only models which allow for contributions to the masses of the $W$ and $Z$ bosons which are not vanishingly small are those that preserve $\rho=1$ at tree level:

i. models with extra $\mathrm{SU}(2)_{L} \operatorname{doublet}(\mathrm{s})$ and/or singlet(s);

ii. a model with an extra $\mathrm{SU}(2)_{L}$ septet with appropriately-chosen hypercharge $[5$, $6]$; and

iii. the Georgi-Machacek (GM) model $[7,8]$ and its generalizations to larger $\mathrm{SU}(2)_{L}$ representations $[9-13]$.

In this thesis we consider the GM model which is the simplest of the allowed 'exotic' models. In addition to the usual $\mathrm{SU}(2)_{L}$ doublet, this model contains two $\mathrm{SU}(2)_{L}$-triplet scalar fields, arranged in such a way that the scalar potential is invariant under a global $\mathrm{SU}(2)_{L} \times \mathrm{SU}(2)_{R}$ symmetry; upon electroweak symmetry breaking, 
this global symmetry breaks down to its diagonal subgroup [known as the custodial $\mathrm{SU}(2)]$ and $\rho=1$ is thereby preserved. The GM model gives rise to a rich and exotic phenomenology, including singly- and doubly-charged scalars that couple to vector boson pairs at tree level and the possibility that the SM-like Higgs boson's couplings to $W W$ and $Z Z$ could be larger than in the SM. In the GM model, the doubly-charged scalars are a part of the so-called custodial-fiveplet $H_{5}^{ \pm \pm}, H_{5}^{ \pm}$, and $H_{5}^{0}$ states, which have a common mass $m_{5}$ and are fermiophobic (meaning they do not have a tree-level coupling to fermions). Their coupling to vector boson pairs is parameterized by $s_{H} \equiv \sin \theta_{H}=2 \sqrt{2} v_{\chi} / v$, where $v_{\chi}$ is the vacuum expectation value (vev) of the isospin-triplet scalars in the GM model and $v$ is the Higgs vev in the SM. In particular, $s_{H}^{2}$ is equal to the fraction of the $W$ and $Z$ boson squared-masses that is generated by the triplet vevs. Assuming that the branching ratios of the $H_{5}$ states to vector bosons are close to 1, the proportionality of the vector boson fusion production cross section of the $H_{5}$ states to $s_{H}^{2}$ allows LHC searches to directly constrain $s_{H}$ as a function of $m_{5} \quad$ [14-20].

The current LHC searches for the $H_{5}$ states in the GM model focus on $H_{5}$ masses of $200 \mathrm{GeV}$ and above, for which the decays into vector boson pairs are on shell. Below this mass, there exists a largely unprobed region in the parameter space with doubly-charged Higgs bosons as light as $120 \mathrm{GeV}$ still allowed. However, this open region of parameter space could easily be constrained by extending existing experimental searches to lower masses. For example, the ATLAS search for DrellYan production of $H^{++} H^{--}$with decays to like-sign $W$ bosons [20], if extended to masses below $200 \mathrm{GeV}$ and using the full Run 2 data-set, would probe this entire neglected low-mass region independent of $s_{H}$ and could potentially entirely exclude $H_{5}$ masses below 200-300 GeV. (The only possible loophole to such an exclusion 
would be mass spectra engineered to make the Higgs-to-Higgs decays $H_{5}^{++} \rightarrow H_{3}^{+} W^{+}$ and/or $H_{5}^{++} \rightarrow H_{3}^{+} H_{3}^{+}$dominate over the $H_{5}^{++} \rightarrow W^{+} W^{+}$decay mode. It is not yet known whether such a scenario can be achieved while satisfying all theoretical and experimental constraints.) To simplify the interpretation of the above searches, the LHC Higgs Cross Section Working Group developed the H5plane benchmark scenario for the GM model [21,22], defined for $m_{5} \in[200,2000] \mathrm{GeV}$. The H5plane benchmark takes as its two free parameters $m_{5}$ and $s_{H}$ and the other parameters are fixed to ensure $B R\left(H_{5} \rightarrow V V\right)=1$ to a good approximation. However, for $m_{5}<200 \mathrm{GeV}$, the H5plane benchmark is unable to cover a significant fraction of the region allowed in a general parameter scan.

In this thesis we propose and study a new benchmark plane in the GM model that is valid in the low- $m_{5}$ region, defined in our case to be $m_{5} \in(50,550) \mathrm{GeV}$. The purpose of this benchmark is to facilitate experimental searches that extend to $m_{5}$ values below $200 \mathrm{GeV}$. This benchmark is designed to yield $B R\left(H_{5} \rightarrow V V\right)=$ 1 while avoiding constraints from the measurements of the $125 \mathrm{GeV}$ Higgs boson signal strengths; in particular, the parameters are chosen to avoid large modifications to $h \rightarrow \gamma \gamma$ from loops of light $H_{5}^{ \pm}$and $H_{5}^{ \pm \pm}$. We investigate the ability of the low- $m_{5}$ benchmark to successfully populate a large portion of the theoretically and experimentally allowed regions and determine the dominant exclusions in the low$m_{5}$ region. We investigate the phenomenology of the benchmark focusing on the behaviour of the SM-like Higgs couplings to fermion and vector boson pairs, $\kappa_{f}^{h}$ and $\kappa_{V}^{h}$; the small region of parameter space in which benchmark specific effects allow $H_{5}$ pairs to be produced via on-shell decays of the heavier custodial-singlet scalar $H$; the branching ratios of the loop suppressed decays of the $H_{5}^{0}$ and $H_{5}^{+}$which become significant at low values of $m_{5}$; and whether the $H_{5}$ width to mass ratios are 
sufficiently small to justify the narrow-width approximation.

Moving past our new benchmark, we turn to the GM model's long standing problem of custodial symmetry violation when we consider effects beyond treelevel [23]. This problem leads one to wonder whether the GM model is a valid effective theory at the energies probed by experiments or whether it is simply a toy theory; albeit one that is an useful experimental benchmark. It also raises questions about whether current experimental constraints on the model can be avoided if one incorporates custodial symmetry violation into the GM model. The source of this problem is the fact that the global $\mathrm{SU}(2)_{R}$ symmetry which is imposed on the scalar potential is explicitly violated by the gauging of hypercharge. This explicit $\mathrm{SU}(2)_{R}$ violation leads to an uncontrolled violation of the custodial symmetry at one-loop. The most relevant manifestation of this problem for our purposes, is that it is not possible to compute a consistent set of renormalization group equations (RGEs) for the Lagrangian parameters of the custodial-symmetric GM model unless one sets the hypercharge gauge coupling to zero [24]. In other words, it is only possible to choose Lagrangian parameters which preserve the custodial symmetry at one particular energy scale. In order to run away from that special scale, one must use the RGEs computed in the full gauge-invariant but custodial-symmetry-violating potential; the hypercharge contribution then causes custodial symmetry violation to accumulate as one runs away from the custodial symmetric scale. Reference [24] independently studied this effect by assuming that the theory is custodial-symmetric at the weak scale and quantifying the amount of custodial symmetry violation that develops as one runs to higher scales.

We take a different approach. We incorporate custodial symmetry violation by assuming that the custodial-symmetric GM model arises at some high scale. Below 
this scale, custodial symmetry violation accumulates through the running of the Lagrangian parameters down to the weak scale. We use weak-scale measurements of the $\rho$ parameter to place upper bounds on the scale of exact custodial symmetry. Subject to this constraint, we also quantify the physical effects of custodial symmetry violation in Higgs-sector observables, such as the ratio of the SM-like Higgs boson couplings to $W W$ and $Z Z$ and custodial-violating mixings, mass splittings among the additional scalars in the GM model, and the induced fermion couplings of the fermiophobic scalars. These results allows us to answer the questions raised by custodial symmetry violation which face the GM model.

This thesis is organized as follows. In Ch. 2 we introduce the SM and briefly describe its relevant features. In Ch. 3 we introduce the GM model before describing and characterizing our proposed low- $m_{5}$ benchmark. In Ch. 4 we introduce the most general gauge invariant extension of the GM model and explore the effect of custodial symmetry violation. In Chapter. 5 we summarize and conclude this thesis. The oneloop RGEs of the GM model and the expressions for triple scalar couplings in the custodial-violating GM theory are presented in the Appendices. 


\section{Chapter 2}

\section{The Standard Model}

From the time the philosophy of Thales whetted the Greeks' thirst for knowledge, the question "So just what is all this stuff made of and why does it act the way that it does?" has vexed and challenged the intellect of man. The modern answer to this ancient question is particle physics. Though young when compared to the great antiquity of the philosophic traditions from which it is derived, particle physics is a mature discipline in its own right. Its birth can be traced to the discovery of the first elementary particle, the electron, by J.J. Thomson in 1897 and its adolescence can be said to have ended with the formulation of the Standard Model of particle physics (SM) in the late 1970s. For reviews of the SM see for e.g. Refs. [4], [25], [26]. The 124 years which have elapsed since the discovery of the electron have allowed ample time for particle physics to reach the equivalent state in its metaphorical life to that of Bilbo Baggins at the beginning of J.R.R. Tolkien's The Lord of the Rings; where it can be said to "possess (apparently) perpetual youth as well as (reputedly) inexhaustible wealth." It's 'youth' is its consistent ability to produce fundamental experimental discoveries decade after decade (many of which were predicted by the SM the most recent of these being the discovery of the Higgs boson in 2012 [27])and its 
'wealth' is the unparalleled agreement between theory and experiment which it boasts (see for e.g. the agreement between theory and experiment in the calculation of the anomalous magnetic dipole moment of the electron [28]). The source of this 'youth' and 'wealth' is the aforementioned SM which consists of 17 (apparently) fundamental particles which makes up all currently known matter and the Quantum Field Theory (QFT) which explains their properties and interactions. The conditional expressions in the above quote are as apt to the current state of particle physics as to an one hundred and eleventy-one years old hobbit; while at present this 'youth' and 'wealth' have proven to be inexhaustible, this is a state of affairs which does not seem likely to continue indefinitely. The discovery of the Higgs boson has completed the experimental verification of the particle content of the SM, meaning new discoveries are no longer as likely as they were in the past. And while experiments have continually agreed with the SM to higher and higher precision it is indisputable that there is important physics beyond the SM (BSM) which should push experiments into significant disagreement with SM predictions. It is the investigation and discovery of BSM physics, both experimentally and theoretically, which is the present goal of both the discipline and this thesis. Before we broach the topic of BSM physics, we will briefly introduce the SM, and elaborate on its features and mechanism which pertain to this thesis.

\subsection{The Particles of the SM}

In the 100 odd years history of particle physics many different sub-atomic particles have been discovered; so many in fact that landscape of the sub-atomic world is often referred to as the particle zoo. Almost all these particles are unstable with very short half-lives which is why everyday matter only consists of the stable particles: electrons, 
protons, and neutrons (free neutrons are unstable but neutrons bound in nuclei are stable). These particles are quantum mechanical objects, possessing properties such as mass and spin as well as various quantum numbers which determine their charge under the fundamental forces of nature (e.g. electric charge) or which can be used to determine their relationship to other particles (e.g. lepton number). Each particle has a corresponding anti-particle (although neutral particles can be their own anti-particles) which can annihilate with the particle into energy or be pair produced from energy. Sub-atomic particles can be divided into two classes: composite particles which are clearly composed of smaller particles (e.g protons and neutrons), and fundamental particles which are apparently not. The main goals of particle physics are to discover all the fundamental particles of nature and to construct a theory to describe their interactions under the fundamental forces of nature. Currently, there are four known forces: electromagnetism, the strong force, the weak force, and gravity. ${ }^{1}$ Electromagnetism and the weak force can be unified into an electro-weak theoretical framework in which they are the product of two different forces known as weak isospin and hypercharge. The SM describes the interactions of particles under the first three of these forces while incorporating the effect of quantum gravity is a challenge that has not yet been met (see Ref. [29] for a review of quantum theories of gravity).

There are 17 discovered particles that are believed to be fundamental [4]. These constitute the entirety of the particles predicted by the SM and are listed along with some of their properties in Table 1.

These 17 particles can be grouped together into different categories. The first division, based on their spin-statistics, is into either fermions or bosons. The fermions are the matter particles, which are the constituents of ordinary matter while the

\footnotetext{
${ }^{1}$ We could claim the existence of a 5 th force, mediated by the Higgs boson but conventionally this is not considered to be a force
} 


\begin{tabular}{|c|c|c|c|c|c|c|}
\hline Particle & Symbol & / distinct antiparticle & Mass & Spin & Charge & Coloured \\
\hline electron & $e$ & yes & $0.5109989461 \pm 0.0000000031 \mathrm{MeV}$ & $1 / 2$ & -1 & no \\
\hline muon & $\mu$ & yes & $105.6583745 \pm 0.0000024 \mathrm{MeV}$ & $1 / 2$ & -1 & no \\
\hline tau & $\tau$ & yes & $1776.86 \pm 0.12 \mathrm{MeV}$ & $1 / 2$ & -1 & no \\
\hline electron neutrino & $\nu_{e}$ & $?$ & $<1.1 \mathrm{eV}$ & $1 / 2$ & 0 & no \\
\hline muon neutrino & $\nu_{\mu}$ & $?$ & $<1.1 \mathrm{eV}$ & $1 / 2$ & 0 & no \\
\hline tau neutrino & $\nu_{\tau}$ & $?$ & $<1.1 \mathrm{eV}$ & $1 / 2$ & 0 & no \\
\hline up quark & $u$ & yes & $2.16_{-0.26}^{+0.49} \mathrm{MeV}$ & $1 / 2$ & $2 / 3$ & yes \\
\hline charm quark & $c$ & yes & $1.27 \pm 0.02 \mathrm{GeV}$ & $1 / 2$ & $2 / 3$ & yes \\
\hline top quark & $t$ & yes & $172.76 \pm 0.30 \mathrm{GeV}$ & $1 / 2$ & $2 / 3$ & yes \\
\hline down quark & $d$ & yes & $4.67_{-0.17}^{+0.48} \mathrm{MeV}$ & $1 / 2$ & $-1 / 3$ & yes \\
\hline strange quark & $s$ & yes & $93.67_{-5}^{+11} \mathrm{MeV}$ & $1 / 2$ & $-1 / 3$ & yes \\
\hline bottom (beauty) quark & $b$ & yes & $4.18_{-0.02}^{+0.03} \mathrm{GeV}$ & $1 / 2$ & $-1 / 3$ & yes \\
\hline photon & $\gamma$ & no & 0 & 1 & 0 & no \\
\hline gluon & $g$ & no & 0 & 1 & 0 & yes \\
\hline W boson & $W$ & yes & $80.379 \pm 0.012 \mathrm{GeV}$ & 1 & -1 & no \\
\hline $\mathrm{Z}$ boson & $Z$ & no & $91.1876 \pm 0.0021 \mathrm{GeV}$ & 1 & 0 & no \\
\hline Higgs boson & $h$ & no & $125.10 \pm 0.14 \mathrm{GeV}$ & 0 & 0 & no \\
\hline
\end{tabular}

Table 2.1: List of all known fundamental particles and some of their properties [4]. All the masses are given in natural units, where $\hbar=c=1$ and the electric charge is given in units of $e$. The anti-neutrinos are distinguished from neutrinos based on their chirality (right-handed versus left handed). It is unknown whether neutrinos and antineutrinos are actually distinct or if they are the same particle (i.e. Dirac or Majorana particles). See for e.g. Ref. [30]. The quarks each carry one of the 3 possible colour charges (red, blue and green) so each type of quark has 3 distinct particles which are otherwise indistinguishable. The gluon carries a colour and anti-colour charge with 8 possible charge assignments, meaning there are 8 gluons. 
bosons mediate the interactions between fermions and are the so-called force carrying particles. The fermions can be divided into two different sectors based on the different charges of the particles under the electro-weak and strong forces. Each sector contains three copies or generations of particles with identical quantum numbers but different masses. These sectors are the lepton sector, which contains the colourless fermions known as leptons (derived from Greek word for small), and the quark sector, which contains the coloured fermions known as quarks (derived from a nonsense word in James Joyce's Finnegan's Wake). The bosons can also be divided into two sectors: the gauge sector which contains the spin-1 vector bosons and the Higgs (or scalar) sector which contains the Higgs boson. The gauge bosons can be further divided based on the force which they mediate. The gluons are the mediators of the strong force while the photon, the $\mathrm{W}$ and the $\mathrm{Z}$ bosons mediate the electro-weak forces. It should also be noted that there are actually more than 17 distinct fundamental particles. This number ignores the corresponding anti-particles which will have the same mass and spin and opposite quantum numbers as the particles. The most famous example of an anti-particle is the positron, identical to an electron in every way with the exception of its +1 charge. Furthermore this number ignores the colour charge of particles. There are three different possible colour charges, red, blue, and green which the quarks can carry. This means that there are three types of every quark; a red, a blue, and a green quark which are otherwise identical. Similarly for gluons, which carry both colour and anti-colour, there are 8 unique colour charges they can carry, meaning there are 8 different types of gluons. Including the anti-particles and different colours gives either 58 or 61 unique fundamental particles depending on whether neutrinos are their own antiparticle.

The primary tool at the disposal of particle physicists to probe the fundamental 
forces of nature, to discover new particles, and study the properties of known particles is the particle collider (for a review of collider physics see Ref. [31]). Colliding either elementary or composite particles together at high energies can produce new particles with a combined mass less than the center-of-mass energy of the collision. The produced particles can be detected, either directly in a detector, if their life time is long enough, or indirectly, by detecting their decay products in a detector; by comparing the rates of detected final particle states to the theoretical predictions the presence of intermediary particles can be inferred. There are two main types of colliders: electron-positron colliders and hadron (i.e. bound states of quarks) colliders. In electron-positron colliders, a high energy beam of electrons is collided with a beam of positrons. The main production mechanism is electron-positron pair annihilation into either a virtual photon or Z boson which will then produce a new real particle anti-particle pair. ${ }^{2}$ Hadron machines collide either two beams of protons or proton-anti proton beams. Since protons are composite particles, there can be interactions between their constituent quarks as well as virtual particles which exist inside the proton. Important particle production mechanisms in a hadron collider include the annihilation of a quark anti-quark pair, referred to as Drell-Yan production, gluon-gluon fusion (GGF) where the interacting particles from each hadron are virtual gluons, and vector boson fusion (VBF) where quarks from each hadron scatter via virtual vector bosons which produce new final state particles. Electron-positron colliders produce cleaner backgrounds but it is more difficult to accelerate electrons and positrons to high energies than protons. Consequently, hadron colliders tend to be used to push the energy frontier to discover new particles, while electron-positron

\footnotetext{
${ }^{2}$ Virtual particles can be thought heuristically as existing as a consequence of the Heisenberg uncertainty principle. They violate the energy-mass relation $E=p^{2} c^{2}+m^{2} c^{4}$ but only exist for a correspondingly short amount of time. Virtual particles that participate in interactions are also referred to as off-shell particles; real particles are referred to as on-shell.
} 
machines tend to be used to study the properties of discovered particles with higher precision.

Unsurprisingly this is exactly the situation today. The current discovery machine is the CERN Large Hardon Collider (LHC) which was designed to push the energy frontier with the goal of discovering the Higgs boson and any new particles with masses at the TeV scale. As stated above, it achieved its first objective, with the ATLAS and CMS collaborations jointly discovering a Higgs boson which is consistent with the SM prediction but did not discover any BSM physics. The LHC has had two runs, the first at a center-of-mass energy of $8 \mathrm{TeV}$ and the second at $13 \mathrm{TeV}$. It is currently undergoing upgrades which will allow it to run at much higher luminosity (HL-LHC) (i.e. allow it to produce more collision events), which will significantly improve its statistics and the precision with which it measures the properties of the discovered Higgs boson and other SM particles [32]. In addition to this upgrade, there are plans in the particle physics community to build a future electron-positron colliders to enable experiments to gather high precision Higgs measurements. The proposed colliders include the International Linear Collider (ILC) [33], which is planned on being built in Japan in the near future, and the more long term proposal of the Future Circular Collider (FCC-ee) [34] which is several decades away from being realised at the earliest.

\subsection{The Theoretical Framework of the SM}

Having briefly discussed the particle content of the SM in the previous section, we will now give a brief and basic overview of the theoretical framework of the SM. The SM can be described very succinctly as a chiral non-abelian gauge quantum field theory with the gauge group $S U(3)_{C} \times S U(2)_{L} \times U(1)_{Y}$ and a field content given in Table 2.2. 


\begin{tabular}{|l|l|l|l|}
\hline Field $(\mathrm{i}=1,2,3)$ & $S U(3)_{C}$ & $S U(2)_{L}$ & $U(1)_{Y}$ \\
\hline$Q_{i}=\left(\begin{array}{c}u_{L i} \\
d_{L i}\end{array}\right)$ & 3 & 2 & $\frac{1}{3}$ \\
\hline$u_{R i}$ & 3 & 1 & $\frac{4}{3}$ \\
\hline$d_{R i}$ & 3 & 1 & $\frac{-2}{3}$ \\
\hline$L_{i}=\left(\begin{array}{c}v_{L i} \\
e_{L i}\end{array}\right)$ & 1 & 2 & -1 \\
\hline$e_{R i}$ & 1 & 1 & -2 \\
\hline$\Phi$ & 1 & 2 & 1 \\
\hline$G$ & 8 & 1 & 0 \\
\hline$W$ & 1 & 3 & 0 \\
\hline$B$ & 1 & 1 & 0 \\
\hline
\end{tabular}

Table 2.2: Field content of the SM and their representations under the gauge group. The gauge forces which are described in the SM are the strong force $\left(S U(3)_{C}\right)$, weak isospin $\left(S U(2)_{L}\right)$, and hypercharge $\left(U(1)_{Y}\right)$. In this thesis we use the convention $Q=T^{3}+\frac{Y}{2}$. At low energies, the gauge symmetry of the $\mathrm{SM}$ is broken and the gauge group breaks down to $S U(3)_{C} \times S U(2)_{L} \times U(1)_{Y} \rightarrow S U(3)_{C} \times U(1)_{E M}$

The details implicit in this description are a broad topic in and of themselves, most of which are not particularly relevant to this thesis. For the sake of brevity the goal of this section is merely to give the general reader a rough idea of what this description of the SM actually means.

\subsubsection{Quantum Field Theory}

We will begin with what is meant by quantum field theory. QFTs are theories which describe physics that involves quantum mechanics, classical field theory and special relativity. The fundamental objects are quantum fields with the particles being the quanta of the quantum fields. The fields can be described using various classical field equations, derived from the classical principle of least action. The action is given by:

$$
S=\int L d t=\int \mathcal{L} d^{4} x
$$


where $L$ is the usual Lagrangian and $\mathcal{L}$ is the Lagrangian density. The action is dimensionless while $\left[d^{4} x\right]=\left[\right.$ length $\left.^{4}\right]$. In natural units $[$ length $]=[\text { energy }]^{-1}$, so $[\mathcal{L}]=[\text { mass }]^{4}$. Since the Lagrangian density is a Lorentz invariant, it is the fundamental building block of QFT and will henceforth be referred to as the Lagrangian instead of $L$. Quantum fields are specified by their transformations under a Lorentz transformation (i.e. they transform as a scalar, a fermion or a vector) which determines their representation under the Lorentz group. The Lorentz representation of a field determines the Lagrangian which describes the non-interacting field theory. Quantum fields can also be described using the usual Hamiltonian formalism of quantum mechanics. The formalism for incorporating fields into quantum mechanics, historically named second quantization, is the simplest way for those familiar with quantum mechanics to conceptually understand these fields although the Lagrangian formalism is generally more useful.

Second quantization begins with representing a field as a normal quantum mechanical wavefunction satisfying some Hamiltonian. The system is quantized by identifying the dynamic variables of the Hamiltonian as operators which obey canonical commutation relations. To incorporate the quantized nature of a field, the wavefunction is also identified as a operator which obeys its own quantization conditions (and can be said to have been quantized again, hence the name second quantization). As a simple illustration of this in the framework of QFT, consider a non-interacting real scalar field, $\phi(x)$. The following discussion follows the framework of Chapter 2 of Ref. [35]. $\phi(x)$ is expressed in momentum space by,

$$
\phi(x, t)=\int \frac{d^{3} p}{(2 \pi)^{3}} e^{i p \cdot x} \tilde{\phi}(p, t)
$$

where $p$ and $x$ are 3 vectors. Consistency with special relativity requires that the 
equation of motion $(\mathrm{EOM}) \phi$ solves is not the Schrodinger equation but rather the Klein-Gordon equation,

$$
\left(\partial^{\mu} \partial_{\mu}+m^{2}\right) \phi=0
$$

Plugging Eq. 2.2 into the Klein-Gordon equation gives,

$$
\left[\frac{\partial^{2}}{\partial t^{2}}+\left(p^{2}+m^{2}\right)\right] \tilde{\phi}(p, t)=0
$$

For a single momentum mode, $p$, this is the just the EOM of a simple harmonic oscillator with $\omega_{p}=\sqrt{p^{2}+m^{2}}$ which can easily be solved using the traditional operator approach from quantum mechanics. Using this same approach to solve the Klein-Gordon Hamiltonian,

$$
H=\int d^{3} x\left[\frac{1}{2} \pi^{2}+\frac{1}{2}(\nabla \phi)^{2}+\frac{1}{2} m^{2}\right],
$$

we first quantize the fields $\phi$ and $\pi$ (the conjugate momentum field) by imposing the canonical commutation relation

$$
[\phi(x), \pi(y)]=i \delta^{3}(x-y)
$$

Following the standard operator approach from quantum mechanics we expand the fields in terms of creation and annihilation operators, $a_{p}$ and $a_{p}^{\dagger}$, where a particle state of momentum $p$ is created by $a_{p}^{\dagger}$ acting on the vacuum state (i.e. $|p\rangle \propto a_{p}^{\dagger}|0\rangle$ ) while $a_{p}|0\rangle=0$. The field operators become:

$$
\phi(x)=\int \frac{d p^{3}}{(2 \pi)^{3}} \frac{1}{\sqrt{2 \omega_{p}}}\left(a_{p}+a_{-p}^{\dagger}\right) e^{i p \cdot x}
$$




$$
\pi(x)=\int \frac{d p^{3}}{(2 \pi)^{3}} \frac{\sqrt{\omega_{p}}}{\sqrt{2} i}\left(a_{p}-a_{-p}^{\dagger}\right) e^{i p \cdot x}
$$

where,

$$
\left[a_{p_{1}}, a_{p_{2}}^{\dagger}\right]=(2 \pi)^{3} \delta^{(3)}\left(p_{1}-p_{2}\right)
$$

Plugging these into the Eq. 2.5 and simplifying gives:

$$
H=\int d p^{3} \omega_{p}\left(a_{p}^{\dagger} a_{p}+\frac{1}{2}\left[a_{p}, a_{p}^{\dagger}\right]\right) .
$$

This is exactly the Hamiltonian for a normal quantum harmonic oscillator with infinite ground state energy. Acting $\phi(x)$ on the ground state $|0\rangle$ gives,

$$
\phi(x)|0\rangle \propto \int \frac{d p^{3}}{(2 \pi)^{3}} \frac{1}{\sqrt{2 \omega_{p}}} e^{i p \cdot x}|p\rangle,
$$

which can be identified with the usual quantum mechanical expression for a position eigenstate. So a quantum field should be thought of as an operator of a simple harmonic oscillator that creates a particle at position $\mathrm{x}$ when it acts on the vacuum.

For an interacting theory, the situation becomes more complicated. The Hamiltonian can no longer be solved exactly but if the interaction coupling constant is small, the interaction terms can be treated as small perturbations of the non-interacting Hamiltonian. In this case, the interacting theory can be expanded using timedependant perturbation theory. This allows for the calculation of approximate n-point correlation functions (e.g. $\langle\omega \phi(x) \phi(y) \mid \omega\rangle$ where $|\omega\rangle$ is the ground state in the interacting theory) scattering and decay amplitudes which are used to predict the scattering cross-section or decay rate of different processes. The amplitude of a given process, also referred to as the invariant matrix element, $\mathcal{M}$ is given by a series expansion in terms of free particle solutions and ordered by power of the interaction coupling 

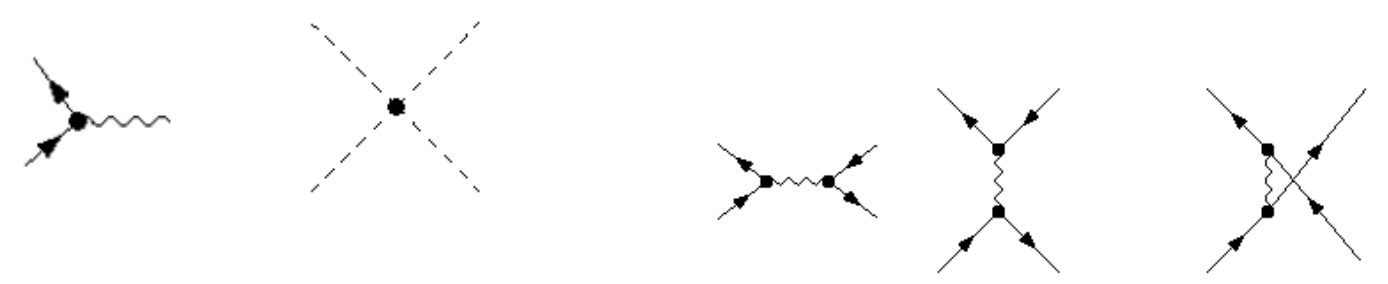

Figure 2.1: Example of tree-level Feynman diagrams. Different particles are conventionally represented by different lines. Straight lines represent fermions (with the direction of the arrow differentiating fermion from anti-fermions), wavey lines represent electro-weak gauge bosons, and curly lines represent gluons. Left: Example vertices from which Feynman diagrams are built. Each vertex represents a rule which can be read from the corresponding interaction term in the Lagrangian. Right: Examples of tree-level Feynman diagrams describing $2 \rightarrow 2$ fermion scattering.

constants. This expansion is rather technical but can be expressed in a very simple form using the prescription of Feynman diagrams. Feynman diagrams represent the perturbative calculation of $\mathcal{M}$ as a series of connected graphs, in which particles are represented by lines and interactions by vertices. They contain both external lines (also referred to as legs) (the initial and final state particles) and internal lines (the virtual particles which are 'exchanged' by the external particles). Each theory defines a given set of Feynman rules which can be read off the Lagrangian of the theory which define the matrix element, $\mathcal{M}_{i}$, of a diagram. A process is represented by all the diagrams in which the external particle lines can be connected using the given vertices. The scattering amplitude of the process is just $\left|\mathcal{M}_{1}+\mathcal{M}_{2}+\ldots \mathcal{M}_{n}\right|^{2}$ meaning there can be constructive and destructive interference between different diagrams. Since the perturbation series is ordered by the power of the interaction coupling constant, the number of vertices that can be used in a diagram determines its order in the perturbation theory. The leading order diagrams typically only involve tree diagrams, so it is conventionally referred to as tree-level or Leading Order (LO). Examples of the dif- 
ferent type of vertices and tree-level diagrams are given in Fig. 2.1. The higher orders are determined by the number of loops the diagrams contain, so the higher orders are referred to by either the number of loops (e.g. 1-loop, 2-loop, etc.) or their relation to the leading order (e.g. Next-to-leading order (NLO), Next-to-Next-to-leading order (NNLO, etc.). Momentum conservation is sufficient to fix all momentum 4-vectors at tree-level (at which level the theory will obey the classical field equations) but this is not the case for loop diagrams. Each loop brings with it an undetermined momentum which needs to be integrated over which introduces effects of higher energy physics into all processes; even low energy processes. This introduces non-classical quantum effects into the field theory which can violate the classical field equations and alter the tree-level relationship between $\mathcal{M}$ and the scattering matrix. These higher order effects are commonly called quantum corrections, quantum effects, loop effects, or loop corrections. In many cases, loop diagrams even contain divergent pieces which need to regularized. These higher order effects can be consistently regularized only if the theory is renormalizable. As will be seen later, renormalizable theories only involve interaction terms with a combined mass dimension of 4 or less. Non-renormalizable terms can be used in calculations but only as an effective field theory (EFT), valid up to some cutoff energy. This cutoff energy is the scale where the true high energy physics becomes important. It usually is related to the masses of the new heavy particles. Since the exact nature of physics above the energies probed in experiments is unknown, modern QFTs are considered in terms of this EFT framework and are considered valid up to some unknown energy scale. See for e.g. Ref. [36]. 


\subsubsection{Chirality and the SM}

Moving on from QFT in general to the SM in particular, it is useful to start with the Lagrangians which describe the matter fields of the SM separately before considering the interaction terms. From the particle content described in the previous sub-section, it is clear there are three types of matter fields in the SM: a scalar bosonic field, spin- $\frac{1}{2}$ fermion fields, and spin-1 bosonic vector fields. The Lagrangians that describe these fields are: the Klein-Gordon Lagragian which for a complex scalar field, $\Phi$ is given by,

$$
\mathcal{L}_{K G}=\left(\partial_{\mu} \Phi\right)^{\dagger}\left(\partial^{\mu} \Phi\right)-m^{2} \Phi^{\dagger} \Phi
$$

(a real field picks up a factor of $\frac{1}{2}$ ); the Dirac Lagragian which for a fermion $\psi$ is given by:

$$
\mathcal{L}_{\text {Dirac }}=\bar{\psi}\left(i \gamma^{\mu} \partial_{\mu}-m\right) \psi
$$

where the $\gamma^{\mu}$ are the anticommuting Dirac matrices, and $\bar{\psi}=\psi^{\dagger} \gamma^{0}$; and the Proca Lagrangian which for a real vector $A^{\mu}$ is given by:

$$
\mathcal{L}_{\text {Proca }}=\frac{1}{4} F^{\mu \nu} F_{\mu \nu}+\frac{m^{2}}{2} A^{\mu} A_{\mu}
$$

where

$$
F^{\mu \nu}=\partial^{\mu} A^{\nu}-\partial^{\nu} A^{\mu}
$$

is the field strength tensor of an abelian field $A^{\mu}$.

Since a Lagrangian has mass dimension 4, the mass dimensions of the fields are given by: $[\phi]=1,[\psi]=\frac{3}{2}$, and $\left[A^{\mu}\right]=1$.

Chirality is a property of fermion fields, $\psi$ which can be thought of as projection which picks out specific components of $\psi$. The chiral projection operators are defined 
as,

$$
P_{L}=\frac{1}{2}\left(1-\gamma^{5}\right), \quad P_{R}=\frac{1}{2}\left(1+\gamma^{5}\right)
$$

where $\gamma^{5}=i \gamma^{0} \gamma^{1} \gamma^{2} \gamma^{3}$. $\gamma^{5}$ is hermitian, anti-commutes with the Dirac matrices and satisfies $\left(\gamma^{5}\right)^{2}=1$. Acting a chiral projection operator on $\psi$ gives a chiral field (referred to as a right-handed or left-handed field respectively) while acting a barred field on a chiral projection operator gives the opposite barred chiral field i.e.

$$
\psi_{L}=P_{L} \psi, \quad \psi_{R}=P_{R} \psi, \quad \bar{\psi}_{R}=\bar{\psi} P_{L}, \quad \bar{\psi}_{L}=\bar{\psi} P_{R}
$$

The Dirac Lagrangian can be re-expressed in terms of chiral fields by using $\left(P_{L}^{2}+\right.$ $\left.P_{R}^{2}\right) \psi=\psi$

$$
\mathcal{L}_{\text {Dirac }}=\bar{\psi}_{L} i \gamma^{\mu} \partial_{\mu} \psi_{L}+{\overline{\psi_{R}}} i \gamma^{\mu} \partial_{\mu} \psi_{R}-m\left(\bar{\psi}_{R} \psi_{L}+\bar{\psi}_{L} \psi_{R}\right)
$$

The kinetic terms only involve either left-handed or right-handed fields but never both while the opposite is true for the mass term. This means that fermions field can be thought of consisting of distinct chiral fields which are mixed together by the Dirac mass term.

\subsubsection{Gauge Symmetry}

The last piece of the puzzle needed to understand the SM as presented at the beginning of this section is the concept of gauge symmetry. With the exception of the Higgs sector, the interaction terms of the SM are given by classically conserved currents. In classical field theory, the relationship between symmetries of the Lagrangian and conserved currents is stated in Noether's theorem which states that every continuous symmetry of the Lagrangian is associated with a conserved current [37]. The most 
famous example of this relationship between symmetry and conserved quantities is conservation of energy and momentum; it can be thought of as a consequence of the invariance of physics under spacetime translations. The principle of gauge symmetry accomplishes the same feat by relating the interactions of particles and the corresponding classically conserved currents as a consequence of geometric rotations in field space. To apply this principle of gauge invariance, we require that the Lagrangian is symmetric under rotations of the local gauge group of the theory. A group is referred to as abelian if its elements commute and non-abelian if they do not. The matter fields themselves are a representation of the gauge group i.e. they are represented as a vector in field space whose components can be rotated into each other by a rotation matrix belonging to the gauge group. For a matter field, $\psi$, this transformation is given by:

$$
\psi(x) \rightarrow V(x) \psi(x)
$$

where $\mathrm{V}(\mathrm{x})$ is an element of the gauge group. It can be written in terms of the generators of the group as [35]

$$
V(x)=e^{i \alpha^{a}(x) t^{a}}
$$

where $\alpha^{a}(x)$ is a function of space-time and $t^{a}$ are the generators of the group.

The requirement that the Lagrangian is invariant under a gauge transformation requires modification of the kinetic terms as the derivative is not gauge invariant. It is replaced by the gauge invariant covariant derivative which encodes the interactions of the matter field. For a given gauge group, the covariant derivative is given by [35],

$$
D_{\mu}=\partial_{\mu}-i g A_{\mu}^{a} t^{a}
$$


where $A_{\mu}^{a}$ is the gauge field associated with the generator $t^{a}$ and $g$ is the coupling constant of the gauge group.

The gauge field $A_{\mu}^{a}$ transforms under a gauge rotation as [35],

$$
A_{\mu}^{a} t^{a} \rightarrow V(x)\left(A_{\mu}^{a} t^{a}+\frac{i}{g} \partial_{\mu}\right) V^{\dagger}(x)
$$

The SM describes 3 different forces each associated with a gauge group: the strong force, with the non-abelian gauge group $S U(3)_{C}$ (the "C" stands for colour) and corresponding gauge field $G_{\mu}^{a}$; weak isospin, with the non-abelian gauge group $S U(2)_{L}$ and gauge field $W_{\mu}^{a}$ that only couples to left-handed fields; and hypercharge with the abelian gauge group $U(1)_{Y}$ and corresponding gauge field $B_{\mu}$. The covariant derivative becomes:

$$
D_{\mu}=\partial_{\mu}-i g W_{\mu}^{a} \tau^{a}-i g^{\prime} \frac{Y}{2} B_{\mu}-i g_{s} G_{\mu}^{a} t^{a}
$$

where $t^{a}$ are the 8 generators of SU(3), known as the Gell-Mann matrices and $\tau^{a}$ are the generators of $\mathrm{SU}(2)$, which for a doublet are given by $\tau^{a}=\frac{\sigma^{a}}{2}$ with

$$
\sigma^{1}=\left(\begin{array}{cc}
0 & 1 \\
1 & 0
\end{array}\right), \quad \sigma^{2}=\left(\begin{array}{cc}
0 & -i \\
i & 0
\end{array}\right), \quad \sigma^{3}=\left(\begin{array}{cc}
1 & 0 \\
0 & -1
\end{array}\right)
$$

being the Pauli matrices. The chiral nature of weak isospin requires that the left and right handed fermions have different representations under the gauge group, the left handed fields transforming as a doublet and the right handed fields as singlets. Having specified the field content and the gauge group, the SM Lagrangian is easy to 
write down. It is given by:

$$
\mathcal{L}_{\text {SM }}=\mathcal{L}_{\text {Gauge }}+\mathcal{L}_{\text {Fermions }}+\mathcal{L}_{\text {Higgs }}
$$

To find the Lagrangian for each sector, we simply write down all the renormalizable gauge invariant terms. For the gauge sector these are:

$$
\mathcal{L}_{\text {Gauge }}=-\frac{1}{4} G^{a \mu \nu} G_{\mu \nu}^{a}-\frac{1}{4} W^{a \mu \nu} W_{\mu \nu}^{a}-\frac{1}{4} B^{\mu} B_{\mu}
$$

where $G^{a \mu \nu}, W^{a \mu \nu}$ and $B^{\mu}$ are the field strength tensors of the gauge fields. Abelian field strength tensors are given in Eq. 2.14 while non-abelian field strength tensors are given by:

$$
G_{\mu \nu}^{a}=\partial_{\mu} G_{\nu}^{a}-\partial_{\nu} G_{\mu}^{a}+g f^{a b c} G_{\mu}^{a} G_{\nu}^{b}
$$

where $f^{a b c}$ are the group theory structure constants defined by:

$$
\left[t^{a}, t^{b}\right]=i f^{a b c} t^{c}
$$

The mass terms which one would expect looking at Eq. 2.13 are not included as they violate gauge invariance.

The allowed terms in the fermion sector are:

$$
\mathcal{L}_{\text {Fermion }}=\bar{L}^{i} i \gamma^{\mu} D_{\mu} L^{i}+\bar{e}_{R}^{\bar{i}} i \gamma^{\mu} D_{\mu} e_{R}^{i}+\bar{Q}^{i} i \gamma^{\mu} D_{\mu} Q^{i}+\bar{u}_{R}^{i} i \gamma^{\mu} D_{\mu} u_{R}^{i}+\bar{d}_{R}^{i} i \gamma^{\mu} D_{\mu} d_{R}^{i}
$$

where the i runs over the three generations. Similarly to the gauge sector the mass terms in Eq. 2.12 also violate gauge symmetry as the left-handed and right-handed fields belong to different representations of the SM gauge group. 
Finally, the allowed terms in the Higgs sector are:

$$
\mathcal{L}_{\text {Higgs }}=\left(D^{\mu} \phi\right)^{\dagger}\left(D_{\mu} \phi\right)-V(\phi)-\left[y_{e}^{i} \bar{L}^{i} \phi e_{R}^{i}+y_{d}^{i} \bar{Q}^{i} \phi d_{R}^{i}+y_{u} \overline{u_{R}^{i}} \tilde{\phi}^{\dagger} Q^{i}+\text { h.c. }\right],
$$

where $V(\phi)$ is the Higgs potential and contains the Higgs mass term (which is gauge invariant unlike the fermion and gauge fields) and the Higgs self interactions. The terms coupling the fermion fields to the Higgs fields define an interaction between fermions mediated by the Higgs; it is normally referred to as the Yukawa interaction. The h.c. stands for hermitian conjugate while the last term is written in terms of the Higgs conjugate field, defined as:

$$
\tilde{\phi} \equiv C_{2} \phi^{*}=\left(\begin{array}{cc}
0 & 1 \\
-1 & 0
\end{array}\right) \phi^{*}=\left(\begin{array}{c}
\phi^{0 *} \\
-\phi^{+*}
\end{array}\right)
$$

To solve the puzzle implied by the total absence of mass terms for every field other than the Higgs boson, given the experimental fact that all the fermion fields and two of the gauge vector boson are massive, requires the introduction of a dynamical mechanism of mass generation. This mechanism is known as the Higgs mechanism and is the most important aspect of the SM for this thesis. The dynamic process of generating mass is referred to as the process of Electro-weak symmetry breaking (EWSB) for reasons which will become clear when we discuss the Higgs mechanism in greater detail in the next section.

\subsection{The Higgs mechanism}

The discussion in this section is based on Ref. [38] and Ch. 20 of Ref. [35]. The Higgs mechanism relies on the fact that the SM treats particles perturbatively i.e. as the 
excitations of a field around the field's minimum value. In most cases the ground state occurs at a field value of zero. This does not need to be the case for the Higgs sector, where the minimum field value is determined by the shape of the Higgs potential. If the potential has multiple valid ground states, the choice to perturbatively expand around a particular one will lead to a breaking of the gauge symmetry. This is referred to as spontaneous symmetry breaking (SSB) since the symmetry is broken as a result of a spontaneous choice in the interpretation of the theory; i.e. it is dynamically broken within the system as opposed to by an external interaction (if the latter is the case we say the symmetry has been explicitly broken).

The SM Higgs potential is given by:

$$
V(\phi)=-\mu^{2} \phi^{\dagger} \phi+\lambda\left(\phi^{\dagger} \phi\right)^{2}=-\mu^{2}|\phi|^{2}+\lambda|\phi|^{4}
$$

where $\phi$ is a complex 2 component vector given by:

$$
\phi=\left(\begin{array}{c}
\phi^{+} \\
\phi^{0}
\end{array}\right) .
$$

The Higgs doublet has 4 independent degrees of freedom. The symmetry of the potential is more readily apparent if we express the Higgs field in terms of its four real components. In terms of real fields, $\phi$ is,

$$
\phi=\frac{1}{\sqrt{2}}\left(\begin{array}{c}
\phi_{1}+i \phi_{2} \\
\phi_{3}+i \phi_{4}
\end{array}\right) .
$$

It is easy to see that Eq. 2.31 can be written in terms of a 4 -d vector in the space of the real Higgs components, $\phi_{v e c}=\frac{1}{\sqrt{2}}\left(\phi_{1}, \phi_{2}, \phi_{3}, \phi_{4}\right)$ (the different normalization is 
a result of the different normalization in the Lagrangians between real and complex scalars), as:

$$
V(\phi)=\frac{-\mu^{2}}{2}\left(\phi_{1}^{2}+\phi_{2}^{2}+\phi_{3}^{2}+\phi_{4}^{2}\right)+\frac{\lambda}{4}\left(\phi_{1}^{2}+\phi_{2}^{2}+\phi_{3}^{2}+\phi_{4}^{2}\right)^{2} .
$$

The potential only depends on powers of $\left|\phi_{v e c}\right|$ (as opposed to $\phi_{v e c}$ ) which means it is invariant under rotations in the 4-d Higgs space. This rotational symmetry corresponds to an $S O(4)$, the special orthogonal group i.e. the group made by all orthogonal $4 \times 4$ matrices with determinant 1 , symmetry.

The minimum of this potential, depends on the sign of the parameters of the potential as is shown in Fig 2.2. If the Higgs self interaction coupling, $\lambda$ is negative, the potential is not bounded from below and any expansion about a local minimum is inherently unstable. If $\lambda$ is positive, there will be either a single global minimum located at $|\phi|=0$ (the unbroken phase where $-\mu^{2}>0$ ) or multiple possible minima (the SSB phase where $-\mu^{2}<0$ ). In the unbroken phase, the gauge bosons and fermions of the SM are massless, in the broken phase, they will acquire a mass if they couple to the Higgs boson. In the broken phase, there is still a combination of gauge rotations which is unbroken (i.e. a combination of rotations that leave the vacuum state invariant). This results in a residual $U(1)_{E M}$ gauge symmetry (which will be identified as the unbroken gauge symmetry of electromagnetism). To reproduce the SM particle spectrum, we require that the potential has a non-zero stable minimum away from zero, which corresponds to the case shown in the top-right panel of Fig. 2.2. This requirement constrains the values of $\mu^{2}$ and $\lambda$ to obey $\lambda>0$ and $\mu^{2}>0$.

To find the value of $|\phi|$ at the minimum of the potential, we minimize Eq. 2.31 

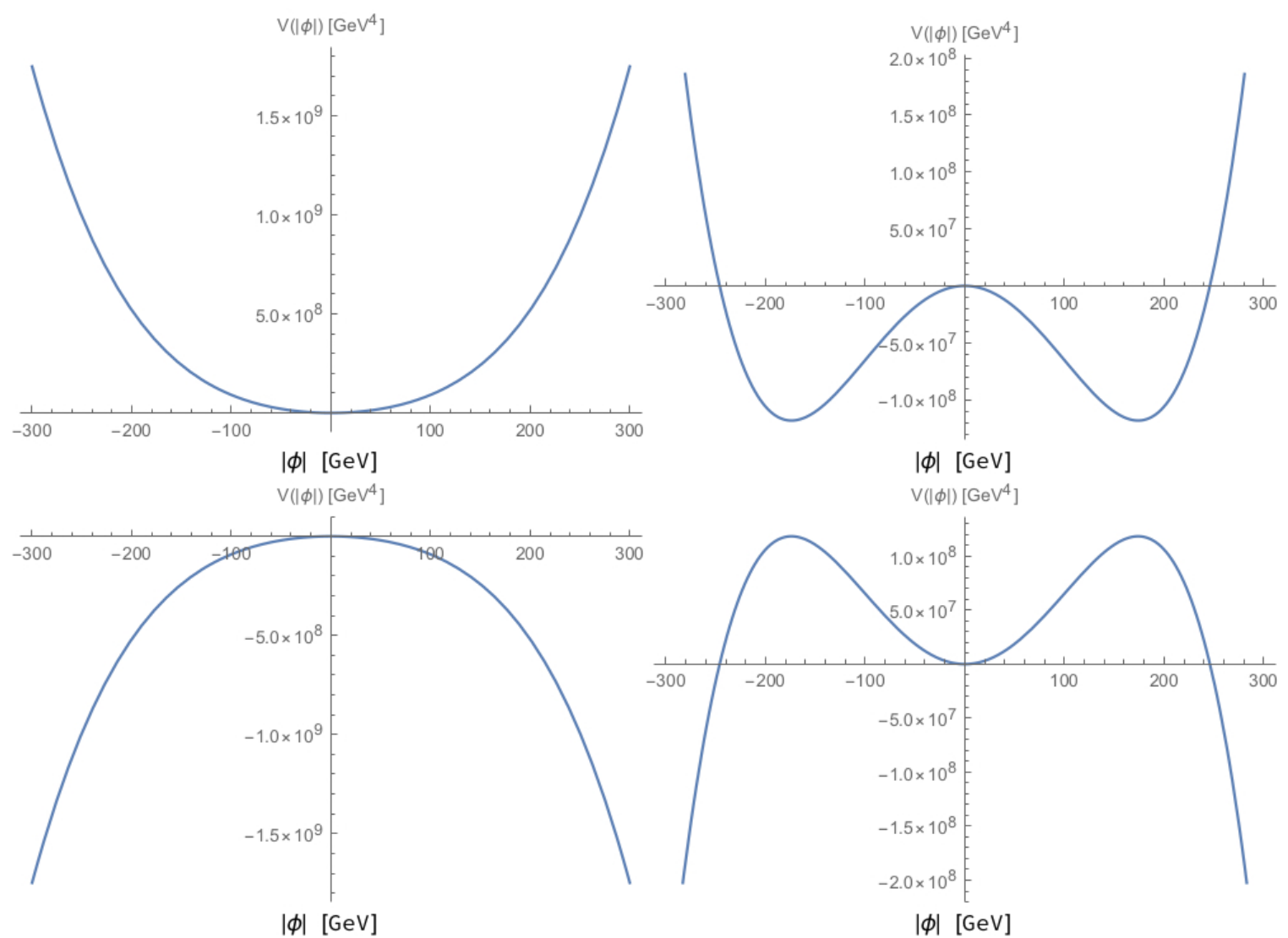

Figure 2.2: The four possible forms of the potential as a function of $|\phi|$. These correspond to positive $-\mu^{2}$ and $\lambda$ (top left), negative $-\mu^{2}$ and positive $\lambda$ (top right), negative $-\mu^{2}$ and $\lambda$ (bottom left), and positive $-\mu^{2}$ and negative $\lambda$ (bottom right) 
with respect to $|\phi|^{2}$. Solving

$$
\frac{\partial V\left(|\phi|^{2}\right)}{\partial\left(|\phi|^{2}\right)}=0=-\mu^{2}+2 \lambda|\phi|^{2}
$$

yields the minimum field value given by:

$$
|\phi|^{2}=\frac{\mu^{2}}{2 \lambda}=\frac{v^{2}}{2}
$$

where $\mathrm{v}$ is known as the vacuum expectation value (vev) of the field. The factor of $\frac{1}{2}$ is conventional. The above equation will be satisfied if the minimum of the field is given by:

$$
\langle\phi\rangle=\frac{v}{\sqrt{2}}
$$

This requirement can easily be met by choosing to give only one component of the Higgs field a non-zero vev. Since we know that the photon does not acquire a mass, we choose to give $\phi_{3}$ a vev as the quantum numbers of the Higgs doublet will ensure the Higgs field does not couple to the photon. To incorporate the vev of $\phi_{3}$ into the theory, we simply introduce a new field, h, defined such that $\phi_{3}=\mathrm{h}+\mathrm{v}$ and $\langle h\rangle=0$. The vevs of the Higgs fields are then given by:

$$
\left\langle\phi_{3}\right\rangle=v \quad\left\langle\phi_{1}\right\rangle=\left\langle\phi_{2}\right\rangle=\left\langle\phi_{4}\right\rangle=\langle h\rangle=0 .
$$

Expanding around the minimum, the Higgs field becomes,

$$
\phi=\frac{1}{\sqrt{2}}\left(\begin{array}{c}
\phi_{1}+i \phi_{2} \\
(h+v)+i \phi_{4}
\end{array}\right) .
$$


and the potential is now given by:

$$
V(\phi)=-\frac{\mu^{2}}{2}\left(\phi_{1}^{2}+\phi_{2}^{2}+(h+v)^{2}+\phi_{4}^{2}\right)+\frac{\lambda}{4}\left(\phi_{1}^{2}+\phi_{2}^{2}+(h+v)^{2}+\phi_{4}^{2}\right)^{2} .
$$

The $S O(4)$ symmetry of the potential has been manifestly broken by the addition of the v term but a residual $S O(3)$ symmetry is still present among the $\phi_{i}$ fields. From Goldstone's theorem, which states that for every spontaneously broken continuous symmetry a theory contains a massless boson [39], we expect the broken theory to contain 1 massive boson and 3 massless Goldstone bosons.

Expanding out the potential gives,

$$
\begin{aligned}
V(\phi) & =-\frac{\mu^{2}}{2}\left(\phi_{1}^{2}+\phi_{2}^{2}+h^{2}+2 h v+v^{2}+\phi_{4}^{2}\right)+\frac{\lambda}{4}\left[\left(\phi_{1}^{2}+\phi_{2}^{2}+\phi_{4}^{2}\right)^{2}\right. \\
& +2 v^{2}\left(\phi_{1}^{2}+\phi_{2}^{2}+\phi_{4}^{2}\right)+2 h^{2}\left(\phi_{1}^{2}+\phi_{2}^{2}+\phi_{4}^{2}\right)+4 v h\left(\phi_{1}^{2}+\phi_{2}^{2}+\phi_{4}^{2}\right) \\
& \left.+h^{4}+6 h^{2} v^{2}+v^{4}+4 v h^{3}+4 h v^{3}\right] .
\end{aligned}
$$

The potential can be simplified by using the minimization condition to eliminate $\mu^{2}$ via $\mu^{2}=\lambda v^{2}$. Defining $\phi_{i}^{2}=\phi_{1}^{2}+\phi_{2}^{2}+\phi_{4}^{2}$ the potential simplifies to,

$$
\begin{aligned}
V(\phi)= & -\frac{\lambda v^{2}}{2}\left(\phi_{i}^{2}+h^{2}+2 h v+v^{2}\right)+\frac{\lambda v^{2}}{2}\left(\phi_{i}^{2}+3 h^{2}\right) \\
& +\frac{\lambda}{4}\left(\phi_{i}^{4}+h^{4}+2 \phi_{i}^{2} h^{2}+4 \phi_{i}^{2} h v+4 h^{3} v+h^{4}+v^{4}\right) \\
= & \lambda v^{2} h^{2}+\frac{\lambda}{4}\left(\phi_{i}^{4}+h^{4}+2 \phi_{i}^{2} h^{2}+4 v \phi_{i}^{2} h+4 v h^{3}\right)-\frac{\lambda}{4} v^{4} .
\end{aligned}
$$

The first term contains the mass terms of the broken theory, the second term the interaction terms, while the last term is an irrelevant constant term which can be ignored. The interaction terms allow us to easily derive another bound on the value 
of the coupling $\lambda$ coming from the requirement of perturbative unitarity of $h h \rightarrow h h$ scattering. To derive this constraint, we begin with QM scattering theory. Recall that the large $r$ form of a wavefunction under the influence of a radial potential can be split into an incoming plane wave and an outgoing spherical [40]: i.e.

$$
\psi(\vec{r}) \rightarrow e^{i \vec{k} \cdot \vec{r}}+f(\theta) \frac{e^{i k r}}{r}
$$

The coefficient of outgoing wave determines the differential cross section and is known as the scattering amplitude. We have:

$$
\begin{aligned}
f(\theta) & =\sum_{j=0}^{\inf }(2 j+1) \frac{S_{j}(k)-1}{2 i k} P_{j}(\cos (\theta)) \\
& =\sum_{j=0}^{\inf }(2 j+1) a_{j} P_{j}(\cos (\theta))
\end{aligned}
$$

where $a_{j}$ is the partial-wave amplitude and the differential cross section is:

$$
\frac{d \sigma}{d \Omega}=|f(\theta)|^{2}
$$

Comparing Eq. 2.45 to the QFT formula for the differential cross section in the center-of-mass frame

$$
\left(\frac{d \sigma}{d \Omega}\right)_{C O M}=\frac{|\mathcal{M}|^{2}}{64 \pi^{2} E_{C O M}}
$$

in the high energy limit where $E_{C O M}=2 p_{C O M}$ and $k=p_{C O M}$ for $2 \rightarrow 2$ scattering of identical particles, we can express $\mathcal{M}$ in terms of the partial waves expansion. I.e.

$$
\mathcal{M}=16 \pi \sum_{j=0}^{\inf }(2 j+1) a_{j} P_{j}(\cos (\theta))
$$


The unitarity of the $S$ matrix requires $\left|S_{l}(k)\right| \leq 1$ which in turn implies:

$$
\begin{aligned}
\left|a_{j}\right| & \leq 1 \\
0 & \leq \operatorname{Im} a_{j} \leq 1 \\
\left|\operatorname{Re} a_{j}\right| & \leq \frac{1}{2} .
\end{aligned}
$$

As we can only calculate $\mathcal{M}$ perturbatively we require that this constraint is satisfied at each order in perturbation theory (hence the name perturbative unitarity). The tree-level S-wave $h h \rightarrow h h$ scattering process (where $j=0$ ) is given by the 4 -scalar diagram shown in Fig. 2.1 which gives: $\mathcal{M}=24 \lambda^{3}$. Eq. 2.47 becomes:

$$
24 \lambda=16 \pi a_{0} .
$$

As the matrix element is real, we can combine the most stringent of the unitarity bounds, Re $\left|a_{0}\right| \leq \frac{1}{2}$ with our previous lower bound from vacuum stability to constrain the value of $\lambda$ to obey $0<\lambda<\frac{\pi}{3}$.

Turning to the mass terms in Eq. 2.42, we can see that the masses of the scalars in the broken phase are:

$$
m_{h}=\sqrt{2 \lambda v^{2}} \quad m_{\phi_{1}}=m_{\phi_{2}}=m_{\phi_{4}}=0 .
$$

The $\mathrm{h}$ field is the SM Higgs field while the three remaining massless fields are the Goldstone bosons. For a broken continuous global gauge symmetry (where the rotation has no space-time dependence) the corresponding Goldstone bosons are real particles. In this case, with a broken local symmetry, the Goldstone bosons

\footnotetext{
${ }^{3}$ The factor of 24 comes from the fact that we have two identical final state particles, two identical initial state particles and 4 ! different ways to connect the external particles to the vertex.
} 
Figure 2.3: The diagram which mixes Goldstone bosons and broken gauge bosons

become quasi-physical and can be gauged away entirely with the appropriate choice of gauge. This is a consequence of the fact that massless vector bosons are transversely polarized, i.e.

$$
p^{\mu} \epsilon_{\mu}=0
$$

where $\epsilon_{\mu}$ is the polarization vector of the vector boson and $p^{\mu}$ is the momentum, and thus have one less degree of freedom (dof) than massive vector bosons. The dof of a Goldstone boson can be traded for a longitudinal dof of a vector boson, in which case the Goldstone boson is said to be 'eaten' by the now massive gauge boson. This can be easily seen by expanding out the Higgs kinetic term,

$$
\begin{aligned}
\mathcal{L}_{\text {Higgs }} \supset\left(D^{\mu} \phi\right)^{\dagger}\left(D_{\mu} \phi\right) & =\partial_{\mu} \phi^{\dagger} \partial_{\mu} \phi+\partial^{\mu} \phi\left(-i g W_{\mu}^{a} \tau^{a}-i g^{\prime} \frac{Y}{2} B_{\mu}\right) \phi+h . c . \\
& +\phi^{\dagger}\left(g \tau^{a} W^{a \mu}+g^{\prime} \frac{Y}{2} B_{\mu}\right)\left(g \tau^{b} W^{b \mu}+g^{\prime} \frac{Y}{2} B_{\mu}\right) \phi .
\end{aligned}
$$

The second term contains trilinear couplings between two scalars and a gauge boson. After SSB this will include terms which couple a single Goldstone boson to a single gauge boson. This is an unphysical coupling, which leads to diagrams of the form shown in Fig. 2.3. These diagrams generate an extra term in the vacuum polarization of the massive gauge bosons which is exactly the missing longitudinal polarization. The unphysical nature of this two particle coupling makes it obvious that it is possible to work in a gauge where the Goldstone bosons don't exist. In this 
gauge, known as the unitarity gauge, the Higgs doublet becomes

$$
\phi=\frac{1}{2}\left(\begin{array}{c}
0 \\
h+v
\end{array}\right)
$$

and the mass terms of the vector bosons are given by:

$$
\begin{aligned}
\mathcal{L}_{\text {Higgs }} & \supset\left(\begin{array}{cc}
0 & v
\end{array}\right)\left(g \tau^{a} W^{a \mu}+g^{\prime} \frac{1}{2} B_{\mu}\right)\left(g \tau^{b} W^{b \mu}+g^{\prime} \frac{1}{2} B_{\mu}\right)\left(\begin{array}{c}
0 \\
v
\end{array}\right) \\
& \left.=\frac{1}{2} \frac{v^{2}}{4}\left[g^{2}\left(W_{\mu}^{1}\right)^{2}+g^{2}\left(W_{\mu}^{2}\right)^{2}+\left(-g W_{\mu}^{3}+g^{\prime} B_{\mu}\right)^{2}\right)\right],
\end{aligned}
$$

plugging in $\mathrm{Y}=1$ for the Higgs doublet and the explicit expressions for the generators. From the terms in the Lagrangian we can write down the SM weak-gauge boson mass mixing matrix as,

$$
M_{\text {gauge }}^{2}=\frac{v^{2}}{4}\left(\begin{array}{cccc}
g^{2} & 0 & 0 & 0 \\
0 & g^{2} & 0 & 0 \\
0 & 0 & g^{2} & -g g^{\prime} \\
0 & 0 & -g g^{\prime} & g^{\prime 2}
\end{array}\right)
$$

(in the basis $\left(W_{\mu}^{1}, W_{\mu}^{2}, W_{\mu}^{3}, B_{\mu}\right)$ ). Diagonalizing this matrix yields three massive and one massless eigenstates. The eigenstates are:

$$
\begin{aligned}
& W_{\mu}^{ \pm}=\frac{1}{\sqrt{2}}\left(W_{\mu}^{1} \mp i W_{\mu}^{2}\right) \quad \text { with mass } \quad m_{W}=g \frac{v}{2} \\
& Z_{\mu}^{0}=\frac{1}{{\sqrt{g^{2}+g^{\prime}}}^{2}}\left(g W_{\mu}^{3}-g^{\prime} B_{\mu}\right) \quad \text { with mass } \quad m_{Z}=\sqrt{g^{2}+g^{\prime 2}} \frac{v}{2} ; \\
& A_{\mu}=\frac{1}{{\sqrt{g^{2}+g^{\prime}}}^{2}}\left(g^{\prime} W_{\mu}^{3}+g B_{\mu}\right) \quad \text { with mass } \quad m_{A}=0 \text {. }
\end{aligned}
$$


In terms of the mass eigenstates, the covariant derivative is given by:

$$
\begin{aligned}
D_{\mu} & =\partial_{\mu}-\frac{g}{\sqrt{2}}\left(W_{\mu}^{+} T^{+}+W_{\mu}^{-} T^{-}\right)-i \frac{1}{\sqrt{g^{2}+g^{\prime}}} Z_{\mu}\left(g^{2} T^{3}-g^{\prime 2} \frac{Y}{2}\right) \\
& -i \frac{g g^{\prime}}{\sqrt{g^{2}+g^{\prime}}} A_{\mu}\left(T^{3}+\frac{Y}{2}\right)
\end{aligned}
$$

where $T^{ \pm}=\left(T^{1} \pm i T^{2}\right)$. For an isospin doublet, this is identical to the QM spin operators $\sigma^{ \pm}$. Since the last term corresponds to the EM current, the electric charge and the charge quantum number are:

$$
e=\frac{g g^{\prime}}{{\sqrt{g^{2}+g^{\prime}}}^{2}} \quad \text { and } \quad Q=T^{3}+\frac{Y}{2}
$$

Under the corresponding EM gauge rotation,

$$
\begin{aligned}
\phi & \rightarrow e^{\alpha\left(\tau_{3}+\frac{1}{2}\right)}\langle\phi\rangle=\exp \left[\left(\begin{array}{ll}
1 & 0 \\
0 & 0
\end{array}\right)\right]\langle\phi\rangle \\
& =\left[\left(\begin{array}{ll}
1 & 0 \\
0 & 1
\end{array}\right)+\sum_{1}^{\infty} \frac{1}{n !}\left(\begin{array}{cc}
1^{n} & 0 \\
0 & 0^{n}
\end{array}\right)\right]\left(\begin{array}{l}
0 \\
v
\end{array}\right) \\
& =\langle\phi\rangle
\end{aligned}
$$

the vacuum state is invariant as expected.

The physical parameters of the electro-weak theory can be expressed in terms of $e$, one of either $m_{W}$ or $m_{Z}$ and the weak mixing angle (also known as the Weinberg angle) which is defined as the angle which rotates the $\left(W^{3}, B\right)$ basis to the $(Z, A)$ basis. i.e.

$$
\left(\begin{array}{c}
Z \\
A
\end{array}\right)=\left(\begin{array}{cc}
\cos \left(\theta_{W}\right) & -\sin \left(\theta_{W}\right) \\
\sin \left(\theta_{W}\right) & \cos \left(\theta_{W}\right)
\end{array}\right)\left(\begin{array}{c}
W^{3} \\
B
\end{array}\right)
$$


where

$$
\cos \left(\theta_{W}\right)=\frac{g}{{\sqrt{g^{2}+g^{\prime}}}^{2}}, \quad \sin \left(\theta_{W}\right)=\frac{g^{\prime}}{{\sqrt{g^{2}+g^{\prime}}}^{2}} .
$$

In terms of e and $\theta_{W}$ the couplings $g$ and $g^{\prime}$ are:

$$
g=\frac{e}{\sin \left(\theta_{W}\right)}, \quad g^{\prime}=\frac{e}{\cos \left(\theta_{W}\right)}
$$

while the masses of the $\mathrm{W}$ and $\mathrm{Z}$ are related via

$$
m_{W}=m_{Z} \cos \left(\theta_{W}\right)
$$

To determine the relationship between the Higgs boson couplings to vector bosons and the vector boson masses, we rewrite Eq. 2.54 in the $W^{ \pm}, Z$ basis. We have:

$$
\begin{aligned}
\mathcal{L}_{\text {Higgs }} & \supset \frac{1}{2}(h+v)^{2}\left[g^{2} W_{\mu}^{+} W^{\mu-}\left(T(T+1)-\left(-\frac{Y}{2}\right)^{2}\right)+Z_{\mu} Z^{\mu} \frac{Y^{2}}{4}\left(g^{2}+g^{\prime 2}\right)\right] \\
& \supset\left(h^{2}+v^{2}+2 h v\right)\left[\frac{g^{2}}{4} W_{\mu}^{+} W^{\mu-}+Z_{\mu} Z^{\mu} \frac{\left(g^{2}+g^{\prime}\right)}{8}\right]
\end{aligned}
$$

using the quatum mechanical identity $T^{+} T^{-}+T^{-} T^{+}=2 T(T+1)-2\left(T^{3}\right)^{2}=1$ and $T^{3}=-\frac{Y}{2}=\frac{1}{2}$ for the neutral component of the Higgs doublet.

The $v^{2}$ terms are the expected mass terms, while the $h^{2}$ and $h v$ terms give $h h V V$ and $h V V$ interaction vertices shown in Fig 2.4 with the following Feynman rules ${ }^{4}$ :

\footnotetext{
${ }^{4}$ The Feynman rule for an interaction is i, the coefficient of the Lagrangian term, a Lorentz factor and combinatorial factor for the number of identical particles in the initial and final states
} 

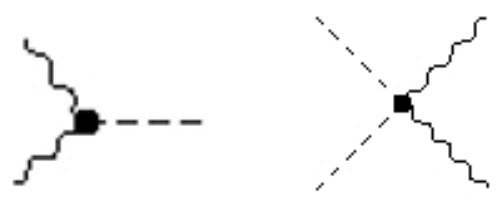

Figure 2.4: The interactions between the massive electro-weak gauge bosons and the Higgs boson generated by EWSB.

$$
\begin{aligned}
h h W_{\mu}^{+} W_{\nu}^{-} & : 2 i \frac{m_{W}^{2}}{v^{2}} g_{\mu \nu} \\
h W_{\mu}^{+} W_{\nu}^{-} & : 2 i \frac{m_{W}^{2}}{v} g_{\mu \nu} \\
h h Z^{\mu} Z^{\nu} & : 2 i \frac{m_{Z}^{2}}{v^{2}} g^{\mu \nu} \\
h Z^{\mu} Z^{\nu} & : 2 i \frac{m_{Z}^{2}}{v} g^{\mu \nu} .
\end{aligned}
$$

As can be seen, the Higgs coupling is always related to the generated mass of a particle.

The fermion masses are generated from the Yukawa terms in very similar manner to the gauge bosons with the added complication that the quark mass basis is not the weak eigenbasis. i.e. the $\mathrm{W}$ bosons couple to linear combinations of the mass eigenstates. This mixing is controlled by the unitary Cabibbo-Kobayashi-Maskawa (CKM) matrix, $V$ elements of which appear in fermion interactions that couple up and down type quarks. The fermion masses are given by:

$$
m_{f}=\frac{y_{f}}{\sqrt{2}} v
$$

where $y_{f}$ is Yukawa coupling of the Higgs boson to the fermion $\mathrm{f}$. 
Alternatively, the Yukawa couplings are given by:

$$
y_{f}=\sqrt{2} \frac{m_{f}}{v} .
$$

\subsubsection{Custodial Symmetry}

A seemingly remarkable feature of the SM is the simple relationship between the masses of the $\mathrm{W}$ and the $\mathrm{Z}$ bosons. Fortunately for model builders, this relationship is not the result of a numerical coincidence but rather it is a consequence of an accidental global symmetry $S U(2)$ of the broken phase of the Higgs potential. This

symmetry is known as Custodial Symmetry (CS) and often denoted $S U(2)_{C}$ [41]. This means CS often plays an important role in extended Higgs sectors (which add additional Higgs fields to the SM) as it guarantees the extended theory reproduces the SM mass prediction without arbitrary tuning of parameters. CS is a symmetry of the Higgs potential but not the full Higgs Lagrangian as it is explicitly broken by both the hypercharge and Yukawa interactions. This CS breaking breaks the mass degeneracy of the massive gauge boson and the up-type and down-type quarks. To see how CS is generated in the SM recall that the scalar potential initially has a global $S O(4)$ symmetry which breaks down to a residual $S O(3)$ after SSB. This symmetry is larger than the one required by EWSB which is of course an $S U(2) \times U(1)$ symmetry which breaks down to a residual $U(1)$. It is quite easy to see how this extra global symmetry becomes a global $S U(2)_{C}$ under EWSB.

Firstly, we note that $S U(2)$ is a double cover of $S O(4)$ i.e.

$$
S O(4) \simeq S U(2) \times S U(2)
$$

so the potential has a global $S U(2)_{L} \times S U(2)_{R}$ symmetry (where the subscripts L and 
$\mathrm{R}$ stand for left and right). To make this symmetry explicit, we re-express Eq. 2.31 in terms of a bi-doublet of $\mathrm{SU}(2)$ given by:

$$
\Phi=\left(\begin{array}{cc}
\phi^{0 *} & \phi^{+} \\
-\phi^{+*} & \phi^{0}
\end{array}\right)
$$

whose columns are simply the conjugate doublet, $\tilde{\phi}$, and the doublet, $\phi$. A $S U(2)_{L} \times$ $S U(2)_{R}$ transformation acting on $\Phi$ is given by:

$$
\Phi^{\prime} \rightarrow e^{i \alpha_{L}^{a} T_{a}} \Phi e^{-i \alpha_{R}^{a} T_{a}}
$$

Eq. 2.31 becomes

$$
V(\Phi)=-\frac{\mu^{2}}{2} \operatorname{Tr}\left(\Phi^{\dagger} \Phi\right)+\frac{\lambda}{2}\left(\operatorname{Tr}\left(\Phi^{\dagger} \Phi\right)\right)^{2}
$$

which is manifestly invariant under a $S U(2)_{L} \times S U(2)_{R}$ transformation. After EWSB the bi-doublet can be written

$$
\Phi_{h}=\left(\begin{array}{cc}
\phi_{h}^{0 *} & \phi^{+} \\
-\phi^{+*} & \phi_{h}^{0}
\end{array}\right)+\frac{v}{\sqrt{2}} I_{2 \times 2}
$$

(where $\phi_{h}^{0}=\frac{h}{\sqrt{2}}+i \operatorname{Im} \phi^{0}$ ) and the potential becomes:

$$
\begin{aligned}
V\left(\Phi_{h}\right) & =-\frac{\mu^{2}}{2}\left[\operatorname{Tr}\left(\Phi_{h}^{\dagger} \Phi_{h}\right)+\frac{v}{\sqrt{2}}\left(\operatorname{Tr}\left(\Phi_{h}\right)+\operatorname{Tr}\left(\Phi_{h}^{\dagger}\right)\right)+v^{2}\right] \\
& +\frac{\lambda}{2}\left(\operatorname{Tr}\left(\Phi_{h}^{\dagger} \Phi_{h}\right)+\frac{v}{\sqrt{2}}\left(\operatorname{Tr}\left(\Phi_{h}\right)+\operatorname{Tr}\left(\Phi_{h}^{\dagger}\right)+v^{2}\right)^{2} .\right.
\end{aligned}
$$

The $S U(2)_{L} \times S U(2)_{R}$ symmetry is broken by terms of the form $\operatorname{Tr}(\Phi)$. These symmetry breaking terms still preserve a residual $S U(2)$ symmetry, corresponding to the rotation where $\alpha_{L}^{a}=\alpha_{R}^{a}$. This symmetry transformation, corresponding to 
the diagonal subgroup of $S U(2)_{L} \times S U(2)_{R}$, is the $S U(2)_{C}$. When we consider the full Higgs Lagrangian, we gauge (i.e. promote $\alpha_{L}^{a}$ to $\left.\alpha_{L}^{a}(x)\right)$ the 3 generators of the global $S U(2)_{L}$ symmetry and one of the global $S U(2)_{R}$ symmetry. This means that the $S U(2)_{L}$ gauge bosons will respect the CS while the hypercharge gauge boson explicitly violates it. Similarly, if the Yukawa couplings $y_{u}^{i}$ and $y_{d}^{i}$ were equal, the quark Yukawa terms become:

$$
\mathcal{L}=-y \bar{Q}^{i} \Phi Q_{R}^{i}+\text { h.c. } \quad \text { where } \quad Q_{R}^{i}=\left(\begin{array}{c}
u_{R}^{i} \\
d_{R}^{i}
\end{array}\right)
$$

Since the up and down Yukawa couplings are not equal, we cannot express the Yukawa sector in terms of $\Phi$, explicitly violating CS [42].

The particles which do respect CS can be arranged according to their representations under the $S U(2)_{C}$. Since CS is an approximate symmetry of the broken theory, this means CS multiplets will be mass degenerate (up to corrections proportional to $\left.g^{\prime}\right)$. The Higgs boson, $h$, transforms as a singlet while the Goldstone bosons and the weak-isospin gauge bosons, $W^{a}$, both transform as triplets under $S U(2)_{C}$ (i.e. they have the same properties under iso-spin rotations as a spin-1 particle under spin rotations). This means that ignoring the effect of hypercharge, the gauge boson mass matrix must take the form,

$$
M_{G}^{2}=\frac{m^{2}}{2}\left(\begin{array}{cccc}
g^{2} & 0 & 0 & 0 \\
0 & g^{2} & 0 & 0 \\
0 & 0 & g^{2} & 0 \\
0 & 0 & 0 & 0
\end{array}\right)
$$

in the $\left(W^{1}, W^{2}, W^{3}, B\right)$ basis. The additional requirement that a sensible vacuum 


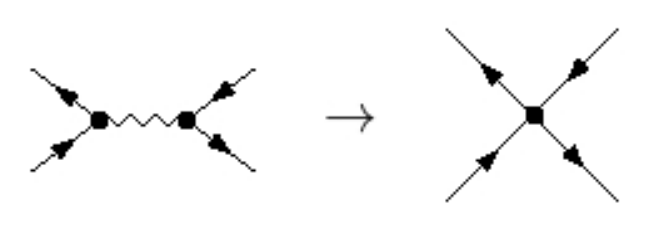

Figure 2.5: An example of an effective 4-Fermi vertex

needs to be invariant under the $U(1)_{E M}$ transformation of $T^{3}+\frac{Y}{2}$ can only be met if the mass mixing matrix has an eigenvalue of zero and if the $W^{1}$ and $W^{2}$ components do not mix with the $W^{3}$ and $B$ components, i.e. the mass mixing matrix defines a 2x2 $W^{3}$ and $B$ mixing matrix where one eigenvalue is zero. This implies the full mass mixing matrix must have precisely the SM form given in Eq. 2.55.

\subsubsection{Low Energy Observables of EWSB}

While the dynamics of EWSB can only be probed at high energy experiments, the energy scale of EWSB and the SM mass relationship between $m_{W}$ and $m_{Z}$ can be probed at low energies. To see this, we consider a non-renormalizable effective theory of fermion interactions, known as the 4-Fermi theory. Historically, 4-Fermi theory was the original theoretical description of the weak charged current interaction which it considered to be a contact interaction between 4 fermions. To derive 4-Fermi theory from the SM, we need to integrate out the heavy degrees of freedom (the $\mathrm{W}$ and $\mathrm{Z}$ bosons). To leading order, this simply means we replace diagrams involving $\mathrm{W}$

exchange by a contact interaction as is shown in Fig 2.5. We start by using Eq. 2.57 to write down the SM fermion interaction terms. These are given by:

$$
\mathcal{L}_{e f f}=-e J_{e m}^{\mu} A_{\mu}-\frac{g}{\sqrt{2}}\left(J_{L}^{+\mu} W_{\mu}^{+}+J_{L}^{-\mu} W_{\mu}^{-}\right)-g_{Z} J_{Z}^{\mu} Z^{\mu}
$$


where

$$
\begin{aligned}
g_{Z}= & \sqrt{g^{2}+g^{\prime 2}} \\
J_{e m}^{\mu}= & -\bar{e} \gamma^{\mu} e+\frac{2}{3} \bar{u} \gamma^{\mu} u-\frac{1}{3} \bar{d} \gamma^{\mu} d \\
J_{L}^{+\mu}= & \overline{\nu_{L}} \gamma^{\mu} e_{L}+\bar{u}_{L} \gamma^{\mu} d_{L} \\
J_{L}^{-\mu}= & \overline{e_{L}} \gamma^{\mu} \nu_{L}+\bar{d}_{L} \gamma^{\mu} u_{L} \\
J_{Z}^{\mu}= & \frac{1}{2} \overline{\nu_{L}} \gamma^{\mu} \nu_{L}+\left(-\frac{1}{2}+\sin ^{2}\left(\theta_{w}\right)\right) \bar{e}_{L} \gamma^{\mu} e_{L}+\sin ^{2}\left(\theta_{w}\right) \bar{e}_{R} \gamma^{\mu} e_{R} \\
& +\left(\frac{1}{2}-\frac{2}{3} \sin ^{2}\left(\theta_{w}\right)\right) \bar{u}_{L} \gamma^{\mu} u_{L}-\frac{2}{3} \sin ^{2}\left(\theta_{w}\right) \bar{u}_{R} \gamma^{\mu} u_{R} \\
& +\left(-\frac{1}{2}+\frac{1}{3} \sin ^{2}\left(\theta_{w}\right)\right) \bar{d}_{L} \gamma^{\mu} d_{L}+\frac{1}{3} \sin ^{2}\left(\theta_{w}\right) \bar{d}_{R} \gamma^{\mu} u_{d}
\end{aligned}
$$

using $g^{2} T^{3}-g^{\prime 2} Y=\left(g^{2}+g^{\prime 2}\right) T^{3}-\left(g^{2}+g^{\prime 2}\right) \sin ^{2}\left(\theta_{w}\right)$ to simplify the Z couplings. In unitarity gauge, the propagators of the $\mathrm{W}$ and $\mathrm{Z}$ are:

$$
\frac{-i}{q^{2}-M_{V}^{2}}\left[g^{\mu \nu}-\frac{q^{\mu} q^{\nu}}{M_{V}^{2}}\right]
$$

where $M_{V}=m_{W}$ or $m_{Z}$. The $\mathrm{W}$ or $\mathrm{Z}$ exchange diagrams in Fig. 2.5 thus have the form

$$
\mathcal{M}=A \bar{\psi}_{1} \gamma^{\mu} \psi_{1} \frac{1}{q^{2}-M_{V}^{2}} \bar{\psi}_{2} \gamma^{\mu} \psi_{2}-A \bar{\psi}_{1} \phi q \psi_{1} \frac{1}{q^{2}-M_{V}^{2}} \bar{\psi}_{2} \not q \psi_{2}
$$

The second term is proportional to $m_{f_{i}}$ since $q=\bar{p}+p$ (from conservation of momentum) and $\bar{\psi}(p) \not p=m \bar{\psi}(p)$ and $\not p \psi(p)=m \psi(p)$ (from the Dirac equation). Using $q^{2}<<M_{V}^{2}$ and $\frac{m_{f}^{2}}{M_{V}^{2}}<<1$, these diagrams are described by an effective Lagrangian:

$$
\mathcal{L}_{e f f}=-\frac{g}{2 m_{W}^{2}} J_{L}^{+\mu} J_{L \mu}^{-}-\frac{g_{Z}^{2}}{2 m_{Z}^{2}} J_{Z}^{\mu} J_{Z \mu} .
$$


These terms describe the weak charged and neutral interactions respectively. Matching this effective Lagrangian to the original 4-Fermi Lagrangian,

$$
\mathcal{L}_{4-F e r m i}=-\frac{4 G_{F}}{\sqrt{2}} J_{L}^{+\mu} J_{L \mu}^{-}-\rho \frac{4 G_{F}}{\sqrt{2}} J_{Z}^{\mu} J_{Z \mu}
$$

and plugging the expression for $m_{W}$ in Eq 2.56 gives an expression for the scale of EWSB, parameterized by the vev $\mathrm{v}$, in terms of the low energy charged current interaction

$$
v^{2}=\frac{1}{\sqrt{2} G_{F}} \approx(246 \mathrm{GeV})^{2},
$$

using the measured value of the Fermi constant:

$$
G_{F}=1.1663787 \times 10^{-5} \mathrm{GeV}^{-2} .
$$

Comparing the relative strengths of the couplings of the low-energy weak neutral and charged currents allows us to probe the ratio of the $\mathrm{W}$ and $\mathrm{Z}$ boson masses. This ratio is known as the $\rho$ parameter [3]. From Eq. 2.81, at leading order $\rho$ is given by:

$$
\rho=\frac{\frac{-\left(g^{2}+g^{\prime 2}\right)}{2 m_{Z}^{2}}}{\frac{-g^{2}}{2 m_{W}^{2}}}=\frac{m_{W}}{\cos \left(\theta_{W}\right) m_{Z}}=1 .
$$

The SM result $\rho=1$ is a consequence of the SM $W$ and $Z$ mass relation which itself is a consequence of the CS of the Higgs potential. The $\rho$ parameter therefore offers a direct experimental probe of the CS of the Higgs sector. Beyond tree-level, it can be measured along with the other electro-weak parameters in the electroweak fit. From the 2020 Particle Data Group fit [4] we have,

$$
\rho=1.00038 \pm 0.00020
$$


which is very close to 1 .

\subsection{Beyond Tree-Level: Renormalized Perturba- tion Theory}

Recall that the fundamental quantities being calculated in QFT, n-point correlation functions and the related scattering cross sections, are calculated perturbatively in terms of the free-theory n-point functions. At tree-level, the scattering amplitudes of the interacting theory can be represented via the exchange of an intermediate virtual particle. The internal virtual particle interacts with one set of the external particles via a vertex given by an interaction Lagrangian term, propagates to the other set of external particles before interacting with them via another interaction vertex. The scattering amplitude of a process can be calculated diagrammatically by applying the appropriate Feynman rules, writing down all possible diagrams which contribute to the process and summing them. Beyond tree-level this simple picture of particle interactions becomes much more complicated. The sum over higher orders of the perturbative expansion involves summing processes with arbitrary numbers of virtual particles. This can be understood heuristically by once again referring to the Heisenberg Uncertainty principle, $\Delta E \Delta t \geq \frac{1}{2}$, which can be thought of as allowing the vacuum to temporarily violate energy conservation by producing any number of sufficiently short-lived virtual particles. This quantum effect allows any particle, both external and internal, to interact with the vacuum as it propagates through space. When these higher order corrections to n-point functions are calculated to a specific order in perturbation theory the calculations can diverge. These divergent integrals can be regularized but this introduces unphysical infinities into QFTs that need to be handled systematically. This systematic process is known as the renormalization 
of a theory.

At a naive level, the divergence of loop calculations can be understood very easily. Each loop a diagram possesses introduces an integration over an undetermined 4-momentum while every virtual particle in a diagram contributes a propagator which contributes negative powers of 4-momentum. I.e. a generic loop integral is of the form:

$$
I \sim \int \frac{d^{4} k_{1} \ldots d^{4} k_{L}}{\not k_{i} \ldots k k_{n}\left(k_{j}^{2}-m^{2}\right) \ldots\left(k_{N}^{2}-m^{2}\right)}
$$

where fermion propagators contribute a factor of $k^{-1}$ while bosonic propagators contribute a factor of $k^{-2}$. Applying the appropriate mathematical tricks, integrals of this form can be expressed in 4-d Euclidean spherical coordinates which means that each loop contributes a factor of $k^{4}$. Naively, the integral will diverge unless the powers of $\mathrm{k}$ in the denominator are greater than the numerator. Using this naive estimation, the superficial degree of divergence, D, of a diagram is given by:

$$
D=4 L-P_{f}-2 P_{b}
$$

where $\mathrm{L}$ is the number of loops, $P_{f}$ the number of fermion propagators and $P_{b}$ the number of bosonic propagators. If we regularize the integral by applying a momentum cutoff $\Lambda$, the integral will diverge as $\Lambda^{D}$ if $D>0, \log (\Lambda)$ if $D=0$ and will be finite if $D<0$. This is only a naive expectation and in practise there are many reasons why a diagram will be more or less divergent than its D would suggest (e.g. symmetry, divergent sub-diagrams, etc.). Since the Feynman rules determine how many loops and propagators are possible for a given diagram, in general D can be expressed as

$$
D=A d+B_{i} V_{i}-C N_{f}-E N_{b}
$$


where $\mathrm{d}$ is the space-time dimension of the theory (i.e. 4 in most cases), $V_{i}$ is the number of vertices of a particular interaction, $N_{f}$ is the number of external fermions and $N_{b}$ is the number of external bosons. The values of the constants A,B,C,E depend on the details of the theory but the dependence on $V_{i}$ allows us to distinguish between three different types of interactions. Firstly if B is negative, D will decrease at higher orders in perturbation theory. Interactions of this type are called superrenormalizable. A theory which consists of only super-renormalizable interactions is called a super-renormalizable theory and it contains a finite number of superficially divergent Feynman diagrams. Secondly if B is zero, D has no dependence on the order in perturbation theory. Interactions of this type are called renormalizable. Theories which contain either super-renormalizable or renormalizable interactions are called renormalizable and contain a finite number of divergent scattering amplitudes but an infinite number of divergent diagrams (the divergent amplitudes will be superficially divergent at all orders). Finally if $\mathrm{B}$ is positive, $\mathrm{D}$ will increase at higher orders of perturbation theory. Interactions of this type are referred to as non-renormalizable. A theory which contains non-renormalizable interactions is called non-renormalizable and all amplitudes will diverge at high enough orders in perturbation theory. As was noted previously in this chapter, an interaction's type can be determined from the mass dimension of its coupling. An interaction will be super-renormalizable, renormalizable, and non-renormalizable if its coupling constant has positive mass dimension, is dimensionless, or negative mass dimension respectively [35].

To illustrate the basic process of renormalization and demonstrate some of the common consequence of renormalized field theory I will briefly describe the renormalization procedure of a toy model, the pseudo-scalar Yukawa theory. The theory contains two fields; a fermion $\psi$ which interacts by exchanging a real pseudo-scalar, 
$\phi$. The classical pseudo-scalar Yukawa Lagrangian is:

$$
\mathcal{L}=\frac{1}{2}\left(\partial_{\mu} \phi\right)^{2}-\frac{1}{2} m^{2} \phi^{2}+\bar{\psi}\left(i \partial_{\mu} \gamma^{\mu}-M\right) \psi-i g \bar{\psi} \gamma^{5} \psi \phi
$$

This Lagrangian describes the Yukawa interaction between a fermion and a pseudoscalar. A pseudoscalar is a scalar field with an odd parity i.e. its discrete parity transformation is $\phi(t, \vec{x}) \rightarrow-\phi(t,-\vec{x})$. When we consider the fermion's parity transformation, $\psi(t, \vec{x}) \rightarrow \gamma^{0} \psi(t,-\vec{x})$, it is clear that the Lagrangian is invariant under a parity transformation and thus that parity is a symmetry of the theory. To renormalize the theory and go from the classical Lagrangian to a quantum Lagrangian, we begin by enumerating the superficially divergent diagrams. In this theory we have

$$
D=4-N_{\phi}-\frac{3}{2} N_{e}
$$

There are seven possible combinations of external fields that give diagrams with $D \geq 0$. These diagrams are given in Fig 2.6.

The first can be ignored as it results in an unphysical shift in the vacuum energy while parity forces the 2 nd and 4 th diagrams to be zero. This leaves 4 diagrams which will possibly diverge at each higher order in perturbation theory. At this point we can already note an important consequence of renormalizing the theory. Diagram vii) corresponds to a four $\phi$ interaction term. In the classical Lagrangian, a $4 \phi$ term could have been written down but as it is not required to describe Yukawa interactions, it can reasonably be ignored at tree-level. But when we consider higher order corrections, we will see that it is actually required to cancel the infinities introduced by loop generated coupling. This means we must include it in the actual Lagrangian of the theory written in terms of the field and couplings that would be measured at 


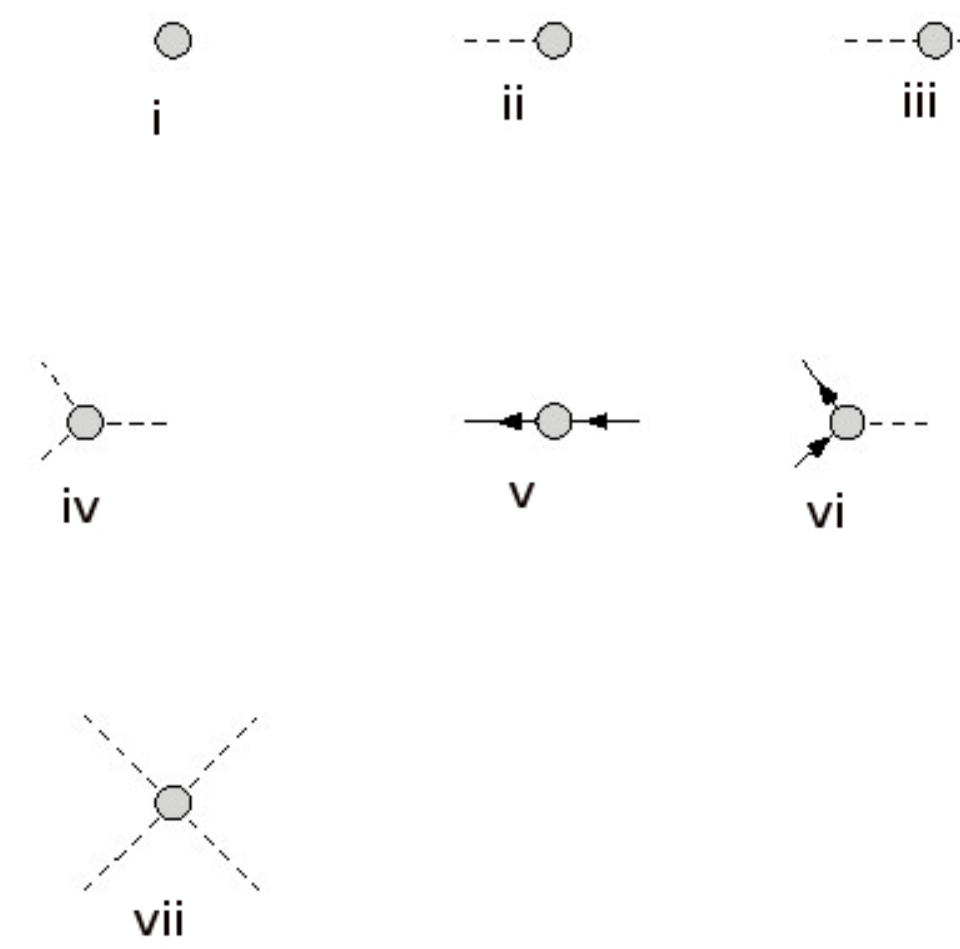

Figure 2.6: The 7 possible superficially divergent diagrams of pseudo-Yukawa theory. 
an infinitely small distance without any vacuum interactions. For this reason, it is known as the bare Lagrangian and its the parameters and fields are called the bare parameters and bare fields. The parameters in the bare Lagrangian are unphysical and cannot be measured. The bare Lagrangian is:

$$
\mathcal{L}=\frac{1}{2}\left(\partial_{\mu} \phi\right)^{2}-\frac{1}{2} m_{0}^{2} \phi^{2}+\bar{\psi}\left(i \partial_{\mu} \gamma^{\mu}-M_{0}\right) \psi-i g_{0} \bar{\psi} \gamma^{5} \psi \phi-\frac{\lambda_{0}}{4 !} \phi^{4}
$$

We begin the renormalization process by considering the effect of higher order corrections on the 2-point functions given by diagrams iii) and v). These diagrams determine the propagators of the particles and the vacuum effects result in the rescaling of the field strength. The full perturbative effect of the vacuum effects on the propagator of the bare fields can be thought of as a geometric series of insertions of all one-particle irreducible diagrams (diagrams which cannot be separated into two different two-point functions by cutting a single line), $M^{2}\left(p^{2}\right)$. If we assume the series converges, the bare scalar propagators become [42]:

$$
\frac{i}{p^{2}-m_{o}^{2}-M^{2}\left(p^{2}\right)}
$$

while the fermion propagators are equivalently modified. In general, the physical propagators will also be rescaled by a factor $\mathrm{Z}$ which will also appear in the scattering matrix of a process. The physical fermion and scalar propagators become:

$$
\begin{aligned}
\text { fermion }: & =\frac{i Z_{2}}{\not p-m_{f}} \\
\text { scalar }: & =\frac{i Z_{3}}{p^{2}-m_{s}} .
\end{aligned}
$$


These shifts in the propagator and scattering matrix can be eliminated by re-defining the fields, a process which is known as field strength renormalization. The field strength renormalization defines the renormalized fields, $\psi_{r}$ and $\phi_{r}$ according to:

$$
\psi=Z_{2}^{\frac{1}{2}} \psi_{r} \quad \text { and } \quad \phi=Z_{3}^{\frac{1}{2}} \phi_{r}
$$

In terms of the renormalized fields, the Lagrangian becomes:

$$
\mathcal{L}=\frac{1}{2} Z_{3}\left(\partial_{\mu} \phi_{r}\right)^{2}-\frac{1}{2} m_{0}^{2} Z_{3} \phi_{r}^{2}+Z_{2} \bar{\psi}_{r}\left(i \partial_{\mu} \gamma^{\mu}-M_{0}\right) \psi_{r}-i g_{0} Z_{2} Z_{3}^{\frac{1}{2}} \bar{\psi}_{r} \gamma^{5} \psi_{r} \phi_{r}-Z_{3}^{2} \frac{\lambda_{0}}{4 !} \phi_{r}^{4}
$$

The infinite contributions to the parameters of the model can be eliminated by splitting the above Lagrangian into finite physical terms and their corresponding counter-terms which contain the infinities present in the bare theory. Defining the counter-terms and the physical couplings to be:

$$
\begin{aligned}
\delta_{3} & =Z_{3}-1 \\
\delta_{m} & =m_{0}^{2} Z_{3}-m^{2} \\
\delta_{\lambda} & =\lambda_{0} Z_{3}^{2}-\lambda \\
\delta_{2} & =Z_{2}-1 \\
\delta_{M} & =Z_{2} M_{0}-M \\
\delta_{g} & =Z_{1}-1=\frac{g_{0}}{g} Z_{2} Z_{3}^{\frac{1}{2}}-1,
\end{aligned}
$$

where we've defined $g Z_{1}=g_{0} Z_{2} Z_{3}$, and plugging them into Eq. 2.95 gives us the 
renormalized Lagrangian

$$
\begin{aligned}
\mathcal{L} & =\frac{1}{2}\left(\partial_{\mu} \phi_{r}\right)^{2}-\frac{1}{2} m^{2} \phi_{r}^{2}+\bar{\psi}_{r}\left(i \partial_{\mu} \gamma^{\mu}-M\right) \psi_{r}-i g \bar{\psi}_{r} \gamma^{5} \psi_{r} \phi_{r}-\frac{\lambda}{4 !} \phi_{r}^{4}+\frac{\delta_{3}}{2}\left(\partial_{\mu} \phi_{r}\right)^{2} \\
& -\frac{1}{2} \delta_{m} \phi_{r}^{2}+\bar{\psi}_{r}\left(i \delta_{2} \partial_{\mu} \gamma^{\mu}-\delta_{M}\right) \psi_{r}-i \delta_{g} \bar{\psi}_{r} \gamma^{5} \psi_{r} \phi_{r}-\frac{\delta_{\lambda}}{4 !} \phi_{r}^{4}
\end{aligned}
$$

The Lagrangian now defines the Feynman rules for pseudo-scalar Yukawa theory with physical parameters with additional rules for the counter-terms. The values of the counter-terms are fixed by requiring that the n-point functions or amplitudes satisfy some physical condition; these renormalization conditions result in equations that fix the values of the counter-terms. The number of renormalization conditions needed is equal to the number of counter-terms. To fix $\delta_{m}, Z_{3}, \delta_{M}$, and $Z_{2}$, we require that the fermion and scalar propagators have poles at $m$ and $M$ respectively. This will result in four conditions. The scalar 1PI function must satisfy:

$$
\left.M^{2}\left(p^{2}\right)\right|_{p^{2}=m^{2}}=0 \quad \text { and }\left.\quad \frac{d}{d p^{2}} M^{2}\left(p^{2}\right)\right|_{p^{2}=m^{2}}=0
$$

with similar conditions on the fermion 1PI function. To fix $\delta_{g}$ and $\delta_{\lambda}$ we require that the induced $\phi \bar{\psi} \psi$ coupling, $g \Gamma$, and the $\phi^{4}, \Gamma^{4 \phi}$ coupling are $g \gamma^{5}$ and $-i \lambda$ at zero momentum transfer respectively.

As a example of the renormalization process, consider the renormalization condition which fixes $\delta_{g}$. The diagrams which contribute to the renormalization at oneloop are given in Fig. 2.7. To separate the effects of wavefunction renormalization, the coupling renormalization conditions only involve vertex corrections, not corrections to the external legs of a diagram. 

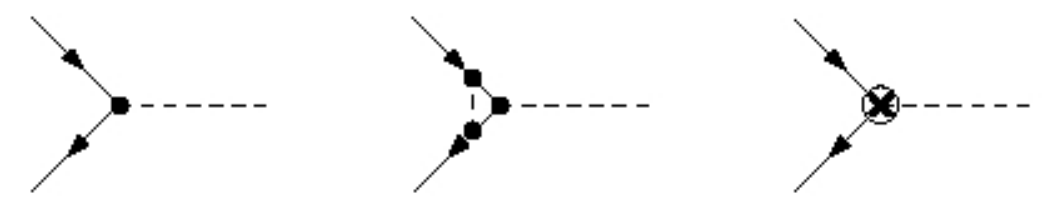

Figure 2.7: The diagrams which contribute to the 1-loop renormalization of the Yukawa coupling g.

At momentum transfer, $\mathrm{q}$, the physical coupling is given by:

$$
g \Gamma(q)=g \gamma^{5}+g \delta \Gamma(q)+g \gamma^{5} \delta_{g}
$$

where $g \gamma^{5}$ is the tree-level contribution, $g \delta \Gamma=\delta F \gamma^{5}$ is the divergent 1-loop contribution and $g \gamma^{5} \delta_{g}$ is the tree-level counter-term contribution. The renormalization condition is satisfied if:

$$
\delta_{g}=-\delta F(0)
$$

The form of the counter-term depends on the method used to regularize the integral. The most common regularization procedure is to replace potentially divergent integrals of the form

$$
I=\int \frac{d l^{4}}{(2 \pi)^{4}} \frac{N\left(l^{2}\right)}{\left(l^{2}-\Delta\right)^{n}}
$$

with an integral over an arbitrary space-time dimension, $d$ i.e.

$$
I \rightarrow \int \frac{d l^{d}}{(2 \pi)^{d}} \frac{N\left(l^{2}\right)}{\left(l^{2}-\Delta\right)^{n}}
$$

The divergent pieces are regularized by defining $d=4-\epsilon$ with divergences appearing 
in the limit where $\epsilon \rightarrow 0$. The divergent pieces are generically of the form

$$
I^{d i v}=\frac{1}{16 \pi^{2}}\left(\frac{2}{\epsilon}-\log (\Delta)-\gamma+\log (4 \pi)+\mathcal{O}(\epsilon)\right)
$$

where $\gamma \approx 0.5772$ is the Euler-Mascheroni constant and the corresponding argument to the log which will account for the dimensionality of $\Delta$ is part of the counter-terms.

Using dimensional regularization the counter-term is:

$$
\delta_{g}=\frac{2 g^{2}}{16 \pi^{2} \epsilon}+\text { finite }
$$

The divergent pieces of the calculation, the terms proportional to $\frac{1}{\epsilon}$, are energy scale independent and therefore the cancellation of the divergence between the loop and the counter-term will not depend on an energy scale. The finite physical coupling, $\mathrm{g} \Gamma(q)$ which is the result of the aforementioned cancellation will however acquire a logarithmic dependence on a ratio of mass and momentum scales which can be large. Using the same procedure the divergent pieces of the counter-terms are:

$$
\begin{aligned}
\delta_{g}^{d i v} & =\frac{2 g^{2}}{16 \pi^{2} \epsilon} \\
\delta_{\lambda}^{d i v} & =\frac{1}{16 \pi^{2} \epsilon}\left(-48 g^{4}+3 \lambda^{2}\right) \\
\delta_{m}^{d i v} & =\frac{1}{16 \pi^{2} \epsilon}\left(-8 g^{2} M^{2}+\lambda^{2} m^{2}\right) \\
\delta_{M}^{d i v} & =-\frac{2 M g^{2}}{16 \pi^{2} \epsilon} \\
\delta_{2}^{d i v} & =-\frac{g^{2}}{16 \pi^{2} \epsilon} \\
\delta_{3}^{d i v} & =-\frac{4 g^{2}}{16 \pi^{2} \epsilon} .
\end{aligned}
$$

The counter-terms $\delta_{m}$ and $\delta_{\lambda}$ are an example of the kind of complications that 
can arise at loop orders. Even if the couplings $\lambda$ and $m$ are set to zero in the Lagrangian, their counter-terms will be non-zero. This means that setting $\lambda$ and $m$ to zero is not natural, as they must be included in the bare Lagrangian and they will generate an effective coupling whose value will vary with the momentum scale of a process.

\subsection{Beyond Tree-Level: Renormalization Group Equations}

The discussion in this section follows Ch. 12 of Ref [35]. A complementary conceptualization of renormalization which naturally incorporates the scale dependence of renormalized parameters is the framework of renormalization group flows. In this approach the physical Lagrangian is considered to be a low-energy effective Lagrangian derived from some high energy bare Lagrangian. The process of integrating out infinitesimal shells of 4-momentum can be described as a continuous transformation of the effective Lagrangian in the space of all possible Lagrangians. These transformations are confusingly referred to as the renormalization group despite not actually forming a group (since they are not invertible). The low-energy theory can be thought of as the flow from the high energy theory defined at momentum scale $p^{2}=\Lambda$ to the momentum scales probed by experiments. Points where the renormalization group flow leaves the Lagrangian unchanged are referred to as fixed points of the theory; the most obvious fixed point is the free field Lagrangian. The effective Lagrangian contains the free-field theory with the addition of local perturbative operators. There are three types of operators that can be added: relevant operators with mass dimension $<d$, marginal operators with mass dimension $=d$, and irrelevant operators with

mass dimension $>d$. The coefficients corresponding to these operators will depend 
on the renormalization group flow and change depending on the distance from the renormalization scale; this scale dependence is often referred to as the 'running' of the coefficient with the renormalization scale. Relevant operators grow as the renormalization group flows and are analogous to super-renormalizable operators in the renormalized perturbation theory framework. Similarly, irrelevant operators shrink and are analogous to non-renormalizable operators. Finally, marginal operators will either grow or shrink depending on the particularities of the loop corrections and are analogous to renormalizable operators. The scale dependence of a coupling under the renormalization group flow is governed by a differential equation, the Renormalization Group Equation (RGE).

The RGEs of a theory can be calculated perturbatively using renormalized perturbation theory. In renormalizad perturbation theory the cutoff scale is taken to infinity so the running coupling constants cannot depend on the cutoff $\Lambda$. Instead they are dependant on the arbitrary renormalization scale, $\mu$, used to fix the counterterms. In this new frame-work, a fusion of both approaches, the renormalization conditions remain unchanged but are no longer the result of some physical requirement. Instead they are applied at a unphysical spacelike momentum $p^{2}=-\mu^{2}$. The renormalization scale dependence of the couplings enters into the theory when one considers the renormalized correlation functions (i.e. a Green's function) dependence on the renormalization scale, given by the Callan-Symanzik equation [43], [44].

As an example, we return to our toy pseudo-Yukawa theory. For simplicity, we take $m=M=0$ (which is a natural choice). The renormalized n-point Green's function is related to the bare Green's function according to:

$$
Z_{2}^{\frac{n}{2}} Z_{3}^{\frac{m}{2}} G^{(n, m)}(\lambda, g, \mu)=G_{\text {bare }}^{(n, m)}\left(\lambda_{0}, g_{0}, \Lambda\right)
$$


where $Z_{2}, Z_{3}, \lambda$ and $g$ are defined as in the previous sub-section and $n, m$ are the number of fermion and scalar fields in $G^{(n, m)}$ respectively. The LHS depends on the renormalized parameters, $\lambda, g$, and the renormalization scale $\mu$ while the RHS depends on the the bare parameters, $\lambda_{0}, g_{0}$, and the cutoff scale $\Lambda$. An infinitesimal shift in the renormalization scale will shift the renormalized couplings and fields by:

$$
\begin{aligned}
& \mu \rightarrow \mu+\delta \mu \\
& \lambda \rightarrow \lambda+\delta \lambda \\
& \phi \rightarrow \phi+\delta \phi=\left(1+\delta \eta_{3}\right) \phi \\
& \psi \rightarrow \psi+\delta \psi=\left(1+\delta \eta_{2}\right) \psi
\end{aligned}
$$

Applying these parameter shifts to Eq. 2.106 and multiplying by $\frac{\mu}{\delta \mu}$ and defining

$$
\beta_{\lambda} \equiv \frac{\mu}{\delta \mu} \delta \lambda \quad \beta_{g} \equiv \frac{\mu}{\delta \mu} \delta g \quad \gamma_{i} \equiv-\frac{\mu}{\delta \mu} \delta \eta_{i}
$$

gives the Callan-Symanzik equation:

$$
\left[\mu \frac{\partial}{\partial \mu}+\beta_{\lambda}(\lambda, g) \frac{\partial}{\partial \lambda}+\beta_{g}(\lambda, g) \frac{\partial}{\partial g}+n \gamma_{2}+m \gamma_{3}\right] G^{(n, m)}=0
$$

The $\beta$ and $\gamma$ functions are dimensionless functions which are the same for every Green's function of the theory. We can calculate the 1-loop $\beta$ functions of our toy massless pseudo-Yukawa theory by writing down the 1-loop renormalized Green function corresponding to the couplings $\lambda$ and $g$ and plugging them into Eq. 2.109. To one loop, the Green's function of $g$ has the following contributions: 


$$
\begin{aligned}
G^{(2,1)} & =\frac{-i}{\not \not 1_{1} p_{2} p_{3}^{2}}\left(g \gamma^{5}+g \delta \Gamma(q)+g \gamma^{5} \delta_{g}\right. \\
& +g \gamma^{5}(\text { terms coming from diagrams with a loop on an external leg } \\
& \left.\left.-2 \delta_{2}-\delta_{3}\right)\right)
\end{aligned}
$$

where there are two diagrams with a loop on an external fermion line and one with a loop on the external scalar line. Since only the counter-terms depend on $\mu$ and $\frac{\partial}{\partial \lambda} G^{(2,1)}=1$ to leading order, plugging $G^{(2,1)}$ into Eq. 2.109 gives:

$$
\mu \frac{\partial}{\partial \mu}\left(g \delta_{g}-g \delta_{2}-g \delta_{3}\right)+\beta_{g}(g, \lambda)+2 g \gamma_{2}+g \gamma_{3}=0
$$

The definition of $\gamma_{i}$ gives:

$$
\begin{aligned}
\gamma_{i} \equiv-\frac{\mu}{\delta \mu} \delta \eta_{i} & =-\frac{\mu}{Z_{i}^{-\frac{1}{2}}} \frac{Z_{i}(\mu+\delta \mu)^{-\frac{1}{2}}-Z_{i}(\mu)^{-\frac{1}{2}}}{\delta \mu} \\
& =\frac{1}{2} \frac{\mu}{Z_{i}} \frac{\partial}{\partial \mu} Z_{i} .
\end{aligned}
$$

Plugging in the 1-loop expression $Z_{i}=1+\delta_{i}$ and rearranging gives:

$$
\beta_{g}(g, \lambda)=\mu \frac{\partial}{\partial \mu}\left(-g \delta_{g}+\frac{1}{2} g \delta_{3}+g \delta_{2}\right)
$$

The expressions for the counter-terms given in Eq. 2.105 can be expressed in terms of the renormalization scale by noting that the $\frac{2}{\epsilon}$ divergence has a one-to-one corre- 
spondence to the logarithmic divergence, $\log \frac{\Lambda}{\mu^{2}}$. The $\beta$ function is therefore:

$$
\begin{aligned}
& \beta_{g}(\lambda, g)=2 g \delta_{g}^{d i v} \frac{\epsilon}{2}-g \frac{\epsilon}{2}\left(2 \delta_{2}^{d i v}+\delta_{3}^{d i v}\right) \\
& \beta_{g}(\lambda, g)=\frac{5 g^{3}}{16 \pi^{2}} .
\end{aligned}
$$

Similarly for $\beta_{\lambda}(\lambda, g)$, we have:

$$
\begin{aligned}
& \beta_{\lambda}(\lambda, g)=2 \delta_{\lambda}^{d i v} \frac{\epsilon}{2}-4 \lambda \frac{\epsilon}{2} \delta_{3}^{d i v} \\
& \beta_{\lambda}(\lambda, g)=\frac{-48 g^{4}+3 \lambda^{2}+8 g^{2} \lambda}{16 \pi^{2}} .
\end{aligned}
$$

The process of calculating the $\beta$ functions of a theory generalizes from the example of pseudo-Yukawa theory very well. For example the 1-loop $\beta$ functions are calculated for a more realistic scalar sector which includes a gauge interaction in Ref. [45]. The general theory they considered consist of a gauge field $A_{\mu}^{a}$ corresponding to some gauge group, $\mathcal{G}$ with a gauge coupling g and structure constants $C^{a b c}$, scalar fields, $\phi_{i}$ belonging to a representation of $\mathcal{G}$ with generators $\theta_{i j}^{a}$, and fermion fields, $\psi_{\alpha}$ belonging to another representation of $\mathcal{G}$ with generators $t_{\alpha \beta}^{a}$. The Lagrangian is given by:

$$
\begin{aligned}
\mathcal{L} & =-\frac{1}{4} F_{\mu \nu}^{a} F^{a \mu \nu}+\frac{1}{2}\left(\mathcal{D}_{\mu} \phi\right)_{i}\left(\mathcal{D}^{\mu} \phi\right)_{i}+i \bar{\psi} \gamma^{\mu} \mathcal{D}_{\mu} \psi-\bar{\psi}_{\alpha} m_{0 \alpha \beta} \psi_{\beta} \\
& -\bar{\psi}_{\alpha} h_{i \alpha \beta} \psi_{\beta} \phi_{i}-V(\phi) .
\end{aligned}
$$

The scalar potential includes terms of the form

$$
V(\phi) \supset \sum_{i j k l} \frac{1}{4 !} f_{i j k l} \phi_{i} \phi_{j} \phi_{k} \phi_{l},
$$

where $f_{i j k l}$ is symmetric under exchange of indices. 
The RGEs for the Yukawa couplings and the quartic scalar couplings are given by Eq. (2.7) and Eq. (2.8) of Ref. [45]. They are:

$$
\begin{aligned}
16 \pi^{2} \frac{d h_{i}}{d t} & =2 h_{m} h_{i} h_{m}+\frac{1}{2}\left(h_{m} h_{m} h_{i}+h_{i} h_{m} h_{m}\right)+2 \operatorname{Tr}\left[h_{i} h_{m}\right] h_{m} \\
& -3\left[2 t^{a} h_{i} t^{a}+S_{2}(F) h_{i}\right] g^{2}+\frac{1}{288 \pi^{2}} f_{i j k l} f_{j k l m} h_{m}, \\
16 \pi^{2} \frac{d f_{i j k l}}{d t} & =f_{i j m n} f_{m n k l}+f_{i k m n} f_{m n j l}+f_{i l m n} f_{m n j k}-12 g^{2} S_{2}(S) f_{i j k l} \\
& +3 g^{4} A_{i j k l}+8 \operatorname{Tr}\left[h_{i} h_{m}\right] f_{m j k l}-12 H_{i j k l},
\end{aligned}
$$

with $t=\log \mu$ where $\mu$ is the energy scale, $S_{2}(F) \delta_{i j}=\left(t^{a} t^{a}\right)_{i j}$ and $S_{2}(S) \delta_{i j}=\left(\theta^{a} \theta^{a}\right)_{i j}$ are group factors, and

$$
\begin{gathered}
A_{i j k l} \equiv\left\{\theta^{a}, \theta^{b}\right\}_{i j}\left\{\theta^{a}, \theta^{b}\right\}_{k l}+\left\{\theta^{a}, \theta^{b}\right\}_{i k}\left\{\theta^{a}, \theta^{b}\right\}_{j l}+\left\{\theta^{a}, \theta^{b}\right\}_{i l}\left\{\theta^{a}, \theta^{b}\right\}_{j k}, \\
H_{i j k l} \equiv \frac{1}{3 !} \operatorname{Tr}\left[h_{i} h_{j}\left\{h_{k}, h_{l}\right\}+h_{i} h_{k}\left\{h_{j}, h_{l}\right\}+h_{i} h_{l}\left\{h_{j}, h_{k}\right\}\right] .
\end{gathered}
$$

The introduction of the gauge interaction and additional fields leads to slightly more complicated $\beta$ functions but the calculation is just as easy to conceptualize. First we identify the divergent diagrams (given in Figs. 2-3 of Ref. [45]) and we compute the appropriate counter-terms; then we apply the Callan-Symanzik equation to the Green's function of the interaction which reduces to a sum of the coefficients of the divergent pieces of the counter-terms. The scale dependence of the couplings is introduced when we formally solve the Callan-Symanzik equation. The solution will include a Green's function which is a function of running couplings, $\lambda$ whose energy dependence is given by RGEs:

$$
\frac{d}{d \log \left(\frac{p}{\mu}\right)} \bar{\lambda}(p)=\beta(\bar{\lambda})
$$


with initial condition $\lambda(\mu)=\lambda$.

This is a non-perturbative result, but in practice, we need to calculate $\beta$ perturbatively as we did above. It is clear that calculating the $\beta$ functions particularly lends itself to algorithmic implementations. This makes the procedure of calculating RGEs (like many calculations involving Feynman diagrams) relatively easy to automate and for most modern applications RGEs are calculated automatically using public codes. Examples of available public codes include: PyR@TE [46], a Python code that generates two-loop RGEs for non-supersymmetric models, and SARAH [47], a Mathematica package which can generate two-loop RGEs for supersymmetric and non-supersymmetric models. The particular details of a model are written in a model card. For some models, the cards are publicly available while others require the user to write their own custom model card.

In the renormalized pseudo-Yukawa theory of the previous sub-section, the loop calculations depended on energy-independent dimensionless couplings. The physical processes described by the theory picked up a scale dependence via logarthimic enhancements generically given by: $\log \left(\frac{p^{2}}{M^{2}}\right)$. These enhancements can eventually grow to the point where the logarithmic enhancements are large enough that the perturbative expansion breaks down. Using the renormalization group picture, these large logarithms are absorbed into the running coupling constants slowly as the energy scale moves away from the renormalization scale. The $\beta$ functions are calculated perturbatively order by order and matched to the renormalized perturbation theory via the initial condition. The initial condition is also calculated perturbatively raising the question how these two perturbative calculations should be matched. In renormalized perturbation theory the higher order corrections take the form: 
Order in Pert. Theory : Correction

$$
\begin{aligned}
\text { Tree-level } & : \text { (none) } \\
N L O & : \quad \alpha \ln \left(\frac{p}{\mu}\right)+\alpha \\
N N L O & : \alpha^{2} \ln \left(\frac{p}{\mu}\right)^{2}+\alpha^{2} \ln \left(\frac{p}{\mu}\right)+\alpha^{2} \\
N^{3} L O & : \alpha^{3} \ln \left(\frac{p}{\mu}\right)^{3}+\alpha^{3} \ln \left(\frac{p}{\mu}\right)^{2}+\alpha^{3} \ln \left(\frac{p}{\mu}\right)+\alpha^{3}(2
\end{aligned}
$$

where $\alpha=\frac{g^{2}}{4 \pi}$.

Using the running coupling constant captures the leading logarithmic contribution to the matrix element at the order in perturbation theory the $\beta$ functions are calculated. To self-consistently capture all terms of the same order when we match the running coupling constant to the renormalized perturbation theory we need to match to the previous order of perturbation theory. This means that if we calculate the RGEs at $N^{n} L O$ in perturbation theory, the initial condition of the RGEs needs to be the value of the renormalized coupling at scale $\mu$ calculated to $N^{n-1} L O$. This matching requirement arises from the fact that matching scale is arbitrary outside of the requirement that it does not introduce large logarithmic corrections. Varying the matching scale with N-1 loop matching introduces an $\mathcal{O}\left(\lambda^{N-1}\right)$ ambiguity into the matching condition which will be cancelled to $\mathcal{O}\left(\lambda^{N}\right)$ in the $N^{n} L O$ RGE calculation [48].

The behaviour of the running coupling is completely determined by the sign of its $\beta$ function. If $\beta<0$, the couplings of the theory will be large at low-energies but small at high energies. In this case, the theory is said to be asymptotically free since the perturbative expansion will always be valid at high energies, although it 
can break down at low-energies. An example of a theory of this type is QCD. On the other hand, if $\beta>0$, the coupling grows with the scale and perturbation theory will break down at high energies. Even worse, the continued running of the coupling can tend to infinity at large but finite values of momentum; this is what is known as a Landau pole. The presence of a Landau pole is definitely a non-physical prediction of the theory and raises the question whether the limit where the cutoff is taken to infinity is even well defined suggesting that new high scale physics is needed.

\subsection{Beyond the SM}

Since its formulation in the 1970s the SM has an incredible track record of impressive theoretical predictions. It can self-consistently describe the interactions of all known particles, many of whose existence it predicted prior to their experimental discovery. The properties of these particles are also consistent with their SM predictions, often with levels of agreement between experimental observations and theoretical predictions that are unheard of in any other field of science. Despite its successes, it is almost entirely certain that the SM is incomplete; that there exists as of yet undiscovered physics which goes beyond the purview of the SM. The search for this physics, often referred to as Beyond the SM (BSM) physics, is the main focus of the current experimental and theoretical efforts of the particle physics community. As this thesis itself is concerned with BSM physics, this subsection will briefly lay out reasons we believe BSM physics exists. The belief in the existence of BSM particle physics is rooted in solutions to both experimental and theoretical puzzles.

The strongest evidence for BSM physics comes from a variety of experimental observations which cannot be explained by SM physics. The best example of unde-

niable evidence for BSM physics is neutrino oscillations. In the SM the neutrinos 
are massless by construction while neutrino oscillations require a mismatch between the neutrino mass (with at least two non-zero masses) and gauge eigenstates [49]. In principle, this can be remedied by little more than a tweak to the SM; simply adding the missing right-handed neutrino fields and giving them mass via the standard Higgs mechanism, i.e. via the Yukawa term

$$
\mathcal{L} \subset-y_{\nu} \overline{\nu_{R}^{i}} \tilde{\phi}^{\dagger} L^{i}+\text { h.c. }
$$

This explanation suffers from several drawbacks. The right handed neutrino is a gauge singlet which would make right-handed neutrinos almost undetectable with their only interaction being the minuscule Yukawa interaction. This means that unless new BSM neutrino interactions (e.g. the discovery of neutrino-less double $\beta$ decay which would prove that neutrinos are Majorana not Dirac fermions) are discovered it is practically impossible to distinguish between the quasi-SM neutrinos and more exotic theories of neutrino mass; theories in which the presence of new heavy particles can naturally explain the large mass hierarchy between neutrinos and all other SM fermions for e.g. via a see-saw mechanism [50]. Hints of BSM physics can also appear as the precision of experiments increase. While no experiment have observed the $5 \sigma$ disagreement with $\mathrm{SM}$ predictions required to claim the discovery of BSM physics, there are several anomalies which are approaching the discovery precision. The most notable are disagreement between the SM prediction and the experimental observation of the anomalous magnetic moment of the muon, which has recently risen to a $4.2 \sigma$ discrepancy [51], and experiments involving b quark decays which test the lepton flavour universality of the SM which has recently reached a $3.1 \sigma$ discrepancy [52].

An astronomical observation which cannot be explained by the SM is dark mat- 
ter. In many situations, astronomical observations of the gravitational interactions of luminous matter in the universe disagree with the predictions of General Relativity and Newtonian gravity. The most famous example is the rotation curves of galaxies [53]. These observations can be explained either by modifying our theories of gravity (e.g. MOND, for a review see Ref. [54]) or the presence of a large amount of non-luminous gravitationally interacting matter i.e. dark matter. While theories of modified gravity cannot be ruled out, observations of velocities and gravitational lensing of clusters of galaxies strongly support the inferred existence of dark matter (see for e.g. the famous Bullet cluster [55]). Furthermore, according to the standard model of cosmology, $\Lambda \mathrm{CDM}$, the universe is composed of approximately $70 \%$ dark energy (an unknown form of energy possible related to vacuum energy), 25\% cold dark matter (i.e. non-relativistic, collisionless matter that does not consist of SM particles) and only 5\% regular baryonic matter [56]. Dark matter is an essential component of $\Lambda \mathrm{CDM}$ ability to describe important cosmological observations such as large scale structure or the acoustic peaks of the cosmic microwave background. A complete theory of particle physics consistent with $\Lambda$ CDM will necessarily involves BSM physics.

The probable existence of BSM physics is also motivated by the existence of theoretical problems in the $\mathrm{SM}$ or $\Lambda \mathrm{CDM}$ cosmology. These theoretical problems differ from experimental evidence in that they can be explained by currently existing theories but only under what seem to be unnatural conditions. That is to say that the theory can describe experiments either by relying on an excessive number of arbitrary parameters or numerical coincidences between parameters. Two of the problems in cosmology which can be explained by BSM physics are the horizon problem and the matter-antimatter asymmetry of the universe. The horizon problem, the observed 
homogeneity of what in principle are causally disconnected pieces of the observable universe, is conventionally solved by positing an early epoch of inflation during which the universe expanded very rapidly (see. for e.g. Ref. [57]). There are various particle physics theories which can explain inflation, all of which require the existence of a BSM particle, the inflaton. There are also several different classes of models that can explain the matter-antimatter asymmetry of the universe (see for e.g. Refs. [58] and [59]). It should be noted that both of these problems disappear if the initial conditions of the universe are sufficiently complex and fine tuned.

Within the SM itself, the biggest theoretical indication of BSM physics is the hierarchy problem. Simple put, the hierarchy problem is the statement that the effective mass of the SM Higgs boson is much smaller than its bare mass. This hierarchy of scales is a consequence of renormalization and requires extreme tuning between the bare Higgs mass and its quantum corrections. Recall that the SM is considered to be an effective theory, valid up to some unknown scale $\Lambda$. Since gravity is the only known physical force which is not explained by the SM, we naively expect that $\Lambda$ should be the scale where the quantum effects of gravity become important. This scale, the Planck scale, can be parametrized by the Planck mass, $M_{P}=1.22 \times 10^{19} \mathrm{GeV}$. The Planck scale fixes the strength of gravity while the weak scale, parameterized by the Higgs vev $v=246 \mathrm{GeV}$, fixes the strength of the weak force. The hierarchy problem arises because our naively expectation would be that these scales are comparable but they differ by 17 orders of magnitude. As a hand-wavy example at 1-loop, the corrections to the Higgs mass from fermion loops are quadratically divergent. This means that if we regularize the divergent integral using a simple momentum scale cutoff, the physical Higgs mass at leading order is given by:

$$
m_{h}^{2}=m_{0}^{2}-\delta m^{2}
$$




$$
\begin{aligned}
& =m_{0}^{2}-\frac{4 y^{2}}{16 \pi^{2}} \Lambda^{2} \\
\mathcal{O}(100 \mathrm{GeV})^{2} & =m_{0}^{2}-\mathcal{O}\left(10^{19} \mathrm{GeV}\right)^{2},
\end{aligned}
$$

where $\mathrm{y}$ is the Yukawa coupling to a given fermion. This equation requires the values of $m_{0}^{2}$ and $\delta m^{2}$ be finely tuned to give the observed weak scale. A more careful calculation with a regulator that does not violate gauge symmetry gives a correction $\delta m^{2}$ with a quadratic dependence on the mass of the particle in the loop. This means that we would expect the scale of EWSB to be the mass scale of the heaviest particle which couples to the Higgs [60]. As was the case with the horizon problem and the matter-antimatter asymmetry problem, it is possible that the hierarchy problem is merely the result of a numerical coincidence in the underlying parameters of the SM. Other motivations for BSM physics include attempts to explain the particle spectrum of the SM, i.e. why are there 3 generations of each type of otherwise identical particle with a large hierarchy in mass and is there a reason for the form of $V_{C K M}$ flavour mixing matrix. At high energies under RGE running the gauge couplings all seem to converge toward similar values. This suggests that the different gauge theories of the SM can be unified into one fundamental grand unified theory (GUT) at a very high scale of $\mathcal{O}\left(10^{16} \mathrm{GeV}\right)$. For a review of GUT see section 115 of Ref [4].

The hierarchy problem affects the Higgs sector but not the fermion or gauge sectors because they are protected from large quantum corrections by the symmetries of the SM. An underlying symmetry of a QFT will be respected to all orders in perturbation theory [60]. If a field obeys a symmetry which is broken either spontaneously or explicitly, its mass will be naturally protected from large vacuum corrections. For example, if we take $m_{f} \rightarrow 0$, the SM has an exact chiral symmetry i.e. the left-handed and right-handed fields become distinct. This protects the fermion fields from large 
quantum corrections and fixes $\delta_{f} \propto m_{f}$ to all loop orders. This gives us an easy potential solution to the hierarchy problem whereby we extend the SM in such a way that generates a larger symmetry that protects the Higgs mass from large quantum corrections. This necessarily requires the addition of new fields to the SM and is one of the primary motivations in the search for BSM physics. There have been many different proposed solutions to the hierarchy problem all of which require the addition of new particles at both TeV scales and much higher scales. Popular solutions include super-symmetry (SUSY) [61] which imposes an additional space time symmetry, the eponymous super-symmetry, relating bosonic and fermionic degrees of freedom. This means that every SM field has an equivalent SUSY partner field which will result in the cancellation of the quadratic divergence in the Higgs mass and also provide valid dark matter candidates. Since these fields have not been observed, SUSY cannot be an exact symmetry meaning this cancellation is not exact and the amount of tuning required depends on the scale of the super-partners. In the early 2000s, SUSY was the dominant paradigm and the LHC was built with the expectation of discovering $\mathrm{TeV}$ scale super partners. In hindsight, this expectation was overly optimistic and the strong constraints from LHC experiments combined with strong constraints from dark matter experiments have made SUSY a less attractive solution to the hierarchy problem. Another popular class of models which solve the hierarchy problem are composite Higgs models (for a review of these types of models see for e.g. Ref. [62]). In these models, inspired by QCD, the Higgs sector consists of composite particles which arise as the result of a new strongly coupled gauge force. As is the case with SUSY, the simplest composite models are ruled out by collider constraints (e.g. technicolour), leading to a somewhat unnatural hierarchy between the composite scale and the weak scale. This is not a problem if the SM-like Higgs boson is identified as 
a pseudo-Nambu-Goldstone boson (a Goldstone boson which acquires a mass proportional to a small explicit symmetry breaking term) as is the case in the Littlest Higgs theories [63]. There are many other proposed explanations to the hierarchy problem (see for e.g. Twin Higgs (for e.g. see Ref. [64]), N-Naturalness [65], etc.).

The BSM solutions to many or all of these problems can be approached in two general ways. We can approach the problem by considering the possible theories defined at some high energy scale (the ultraviolet (UV) scale) which results in an experimentally testable low energy effective theory in the low energy limit (the infrared (IR)). Alternatively, we can approach the problem by constraining the low energy effective theories which can then be matched to a UV completion. In general, the solutions to these problems will involve extended Higgs sectors in the IR which will contribute to EWSB. This makes the dynamics of EWSB one of the best probes of BSM at our disposal. To investigate the dynamics of EWSB we need to study the possible extensions of the SM Higgs mechanism. By searching for new Higgs bosons and by measuring the properties of the (apparently) SM-like Higgs boson and comparing them to low-energy effective theory, we can constrain and possibly discover the nature of BSM physics. 


\section{Chapter 3}

\section{The Georgi-Machacek Model}

In the previous chapter, we argued that there are compelling reasons to believe in BSM physics. The theoretical solutions to problems that motivate this belief in BSM physics generally involve extended Higgs sectors. Though many of these solutions focus on the physics of UV completions, in the IR they will produce effective theories which can give different predictions for the dynamics of EWSB. Precision measurements of EWSB will allow us to constrain and eventually discover the correct IR theory. The allowed IR theories can then be used to constrain different UV completions by matching them to the UV scale. At present, experimental probes of EWSB are consistent with the SM and there are many different possible IR effective models of EWSB which are not excluded by current constrains. In the search for new physics, it is important that we have a variety of theories which exhaustively cover the IR BSM possibilities. We need theories to guide experimental searches for new particles and which can explain any BSM properties of known particles which might be revealed as experiments become more precise. To achieve this, theorists need to build theories which can access the full range of currently allowed BSM properties and possibilities. Then as the precision of experiments increase, we can constrain 
and exclude the possible IR models until the surviving theories converge toward the true theory of nature. The process of building, constraining and excluding models is complicated by the ease with which constraints can be avoided by arbitrary tuning of parameters or by constructing increasingly complex models with additional freedom in their parameter space. While this process of model building and model tuning cannot go on indefinitely, it ensures that there are a veritable host of potentially valid BSM theories which need to be tested. In order to tackle this multitude of possibly theories, it is very useful to consider benchmark theories which give the minimal implementation of some new feature of EWSB while avoiding excessive tuning of their parameters.

The number of different possibilities for these benchmark models is quite small. The gauge invariance of the fermion mass terms can only come from a Higgs doublet so all extended Higgs sectors will contain the usual SM doublet. The first class of minimal benchmark models therefore extends the SM Higgs sector by adding a single additional Higgs field to the SM doublet. This field could be: a SM gauge singlet, an isospin doublet, or possibly some higher isospin representations. Each possibility gives us some unique predictive power. The simplest case is the addition of a gauge singlet to the SM. This is known as the scalar singlet model [66-68]. The singlet cannot contribute to the masses of the gauge bosons or the fermions but it will mix with the Higgs doublet, introduce a new neutral Higgs particle to the spectrum and modify the SM-like Higgs couplings. It is used as a prototype of models which use the Higgs interaction as a portal to a hidden sector of particles that are singlets under SM gauge charges. The next simplest case is the well studied Two Higgs Doublet Model (2HDM) (for a review see Ref. [69]) which adds an additional doublet to the SM. Unlike the scalar singlet model, the 2HDM includes a charged Higgs boson and can 
contribute to both the fermion masses and the gauge boson masses. The Higgs sector predicted by the simplest implementation of SUSY, the Minimal Supersymmetric Standard Model is a type II 2HDM (where one doublet couples to the leptons and $\mathrm{d}$ type quarks while the other couples to the $\mathrm{u}$ type quarks). Finally we have the possibility of a contribution from higher isopin multiplets. The prototype for these models is the Higgs triplet model which can give mass to neutrinos via a Type II Seesaw mechanism ${ }^{1}[70]$. Unlike the singlet and $2 \mathrm{HDM}$, the triplet can only give mass to the gauge bosons (with the already noted possible exception of neutrinos), will include a doubly-charged Higgs boson, and can enhance the couplings of the SMlike Higgs boson. The triplet vev, and indeed the vevs of any generic higher isospin multiplets, is strongly constrained by the $\rho$ parameter to be small. To explore the possibility where a significant fraction of the gauge boson masses is not generated by a doublet we need to turn to more complicated models which allow us to avoid these constraints. The simplest model which allows for triplets with relatively large vevs without relying on tuning is the Georgi-Machacek (GM) model, the focus of this thesis. Before we introduce the GM model, it will be instructive to examine in more detail what makes higher isospin extensions of the SM theoretically interesting and what makes the GM model such a useful benchmark model.

\footnotetext{
${ }^{1}$ The Type II see-saw mechanism takes advantage of the fact that the addition of a $Y=2$ triplet allows us to write down a Yukawa term coupling two lepton doublets to the triplet. After EWSB, this term can generate a Majorana mass term for the neutrinos proportional to the triplet vev. In scenarios where the triplet vev is naturally small this leads to a naturally tiny neutrino mass. This term also leads to lepton-number-violating triplet decays to leptons which can be used to constrain these models. In the models considered in this thesis with larger triplet vevs this is not the case. To generate neutrino masses, we require an extremely tiny Yukawa coupling and hence the corresponding lepton-number-violating decays are negligibly small.
} 


\subsubsection{Higher Isospin Extensions of the SM}

In principle, higher isospin multiplets could be a significant contributor to the masses of the $\mathrm{W}$ and $\mathrm{Z}$ gauge bosons. We can set a rough limit on the amount of the gauge boson masses which can be generated by higher isospin multiplets using the fact that the top Yukawa needs to be perturbative. I.e.

$$
4 \pi \geq y_{t}^{2}=\frac{2 m_{t}^{2}}{c_{H}^{2} v^{2}}
$$

where $c_{H}=\frac{v_{\phi}}{v}$ is the fraction of the $\mathrm{W}$ squared mass which is generated by doublets; $v_{\phi}$ is the vev of the doublet field while $\mathrm{v}$ is defined by the Fermi constant as in the SM. Rearranging and plugging in values gives the lower bound:

$$
c_{H}^{2} \geq 0.08
$$

The corresponding upper bound on the mass fraction of $\mathrm{W}$ and $\mathrm{Z}$ masses from higher isospin multiplets is $92 \%$ which immediately makes models that can achieve a significant mass fraction compelling BSM scenarios. Additionally, these models possess unique signatures which cannot be reproduced in singlet or doublet extensions. They possess an unique particle spectrum which includes "exotic" multi-charged Higgs particles not found in singlet and doublet extensions. They can also lead to unique modifications of the SM-like Higgs boson couplings to vector bosons. Recall from Eq 2.64 that the general couplings of a real neutral component of a Higgs field with isospin $\mathrm{T}$ and hypercharge $\mathrm{Y}$ are:

$$
C_{H H W W}=\frac{g^{2}}{2}\left[T(T+1)-\frac{Y^{2}}{4}\right] \quad \& \quad C_{H H Z Z}=\frac{Y^{2}}{8}\left(g^{2}+g^{\prime 2}\right) .
$$


The physical mass eigenstates are linear combinations of the real components of the different Higgs fields which contribute to EWSB. The couplings of the physical Higgs is thus the sum of the couplings of the individual fields multiplied by a mixing angle. Since singlets do not couple to gauge bosons and the only possible doublet hypercharge assignments that can result in a neutral component are $Y= \pm 1$, singlet and doublet extensions will always result in a coupling that is less than or equal to the SM prediction. Since the coupling of higher isospin fields is larger than the SM, these extensions can result in a coupling that is larger than the SM value. Couplings of the discovered Higgs which are larger than the SM have not been ruled out. This means higher isospin extensions allow us to explain the full allowed range of current coupling measurements of the discovered Higgs boson unlike doublet and singlet extensions.

In general these models are very strongly constrained by the low-energy EWSB observable, the $\rho$ parameter. Using the general expression given in Eq 2.64, the tree-level expression for the contribution of $\mathrm{k}$ multiplets to the $\rho$ parameter is:

$$
\rho=\frac{m_{W}^{2}}{c_{W}^{2} m_{Z}^{2}}=\frac{\sum_{k} 2\left(T_{k}\left(T_{k}+1\right)-\frac{Y_{k}^{2}}{4}\right) v_{k}^{2}}{\sum_{k} Y_{k}^{2} v_{k}^{2}}
$$

where the subscript $\mathrm{k}$ specifies a given isospin multiplet. The precise measurement of $\rho$ constrains the vevs of higher isospin multiplets to be negligibly small. This constraint can only be avoided by a cancellation in the contributions to $\rho$. This cancellation can be accidental, the result of tuning of parameters, or the product of a symmetry of the theory. The possibility of higher isospin contributions can also be constrained by the requirement of perturbative unitarity of two-to-two scalar scattering amplitudes i.e. that the $\mathrm{J}=0$ mode of the partial wave expansion of the scattering matrix, $\left|a_{0}\right|<1$. The requirement of perturbative unitarity of vector boson scattering will also place limits on the possible masses of Higgs bosons [71,72]. 
Perturbative unitarity constraints require $T<\frac{7}{2}(T<4)$ for the addition of a single complex (real) scalar [73]. These constraints on isospin are stronger if additional multiplets are added. These two constraints imply that only a limited number of models can involve higher isospin mutliplets and that the only ones which can have a significant contribution to EWSB without resorting to tuning are those which preserve $\rho=1$ at tree level:

i. a model with an extra $\mathrm{SU}(2)_{L}$ septet with appropriately-chosen hypercharge $[5$, $6]$; and

ii. models which add multiple n-plets and impose custodial symmetry. These are the GM model $[7,8]$ and its generalizations to larger $\mathrm{SU}(2)_{L}$ representations $[9-$ $13]$.

All of these models share the unique features associated with higher isospin extensions of the SM. Since the GM model is the simplest of these models as well as the least constrained, it is very useful as a benchmark model for collider experiments searching for exotic signals.

\subsection{The Model}

The scalar sector of the GM model $[7,8]$ consists of the usual complex doublet $\left(\phi^{+}, \phi^{0}\right)$ with hypercharge $Y=1$, a real triplet $\left(\xi^{+}, \xi^{0},-\xi^{+*}\right)$ with $Y=0$, and a complex triplet $\left(\chi^{++}, \chi^{+}, \chi^{0}\right)$ with $Y=2$. As noted above, the doublet is responsible for the fermion masses as in the SM. To ensure that the theory is custodial symmetric we require that the scalar potential possesses the $\mathrm{SU}(2)_{L} \times \mathrm{SU}(2)_{R}$ which was accidentally present in the SM. In order to make the global $\mathrm{SU}(2)_{L} \times \mathrm{SU}(2)_{R}$ symmetry explicit, we write the 
potential in terms of the bidoublet $\Phi$ and combine the triplets to form a bitriplet $X$ :

$$
\Phi=\left(\begin{array}{cc}
\phi^{0 *} & \phi^{+} \\
-\phi^{+*} & \phi^{0}
\end{array}\right), \quad X=\left(\begin{array}{ccc}
\chi^{0 *} & \xi^{+} & \chi^{++} \\
-\chi^{+*} & \xi^{0} & \chi^{+} \\
\chi^{++*} & -\xi^{+*} & \chi^{0}
\end{array}\right)
$$

As in the SM, upon EWSB, the global $\mathrm{SU}(2)_{L} \times \mathrm{SU}(2)_{R}$ symmetry breaks down to the diagonal subgroup, which is the custodial SU(2) symmetry. This ensures that the GM model reproduces the SM gauge boson mass relation giving $\rho=1$ at tree-level. The vevs of the fields are defined by $\langle\Phi\rangle=\frac{v_{\phi}}{\sqrt{2}} I_{2 \times 2}$ and $\langle X\rangle=v_{\chi} I_{3 \times 3}$, where $I$ is the appropriate identity matrix. Matching to the Fermi constant requires the vevs obey

$$
v_{\phi}^{2}+8 v_{\chi}^{2} \equiv v^{2}=\frac{1}{\sqrt{2} G_{F}} \approx(246 \mathrm{GeV})^{2}
$$

The most general gauge-invariant scalar potential involving these fields that conserves custodial $\mathrm{SU}(2)$ is given, in the conventions of Ref. [74], by ${ }^{2}$

$$
\begin{aligned}
V(\Phi, X)= & \frac{\mu_{2}^{2}}{2} \operatorname{Tr}\left(\Phi^{\dagger} \Phi\right)+\frac{\mu_{3}^{2}}{2} \operatorname{Tr}\left(X^{\dagger} X\right)+\lambda_{1}\left[\operatorname{Tr}\left(\Phi^{\dagger} \Phi\right)\right]^{2}+\lambda_{2} \operatorname{Tr}\left(\Phi^{\dagger} \Phi\right) \operatorname{Tr}\left(X^{\dagger} X\right) \\
& +\lambda_{3} \operatorname{Tr}\left(X^{\dagger} X X^{\dagger} X\right)+\lambda_{4}\left[\operatorname{Tr}\left(X^{\dagger} X\right)\right]^{2}-\lambda_{5} \operatorname{Tr}\left(\Phi^{\dagger} \tau^{a} \Phi \tau^{b}\right) \operatorname{Tr}\left(X^{\dagger} t^{a} X t^{b}\right) \\
& -M_{1} \operatorname{Tr}\left(\Phi^{\dagger} \tau^{a} \Phi \tau^{b}\right)\left(U X U^{\dagger}\right)_{a b}-M_{2} \operatorname{Tr}\left(X^{\dagger} t^{a} X t^{b}\right)\left(U X U^{\dagger}\right)_{a b} .
\end{aligned}
$$

Here the $\mathrm{SU}(2)$ generators for the doublet representation are $\tau^{a}=\sigma^{a} / 2$ with $\sigma^{a}$ being

\footnotetext{
${ }^{2} \mathrm{~A}$ translation table to other parameterizations in the literature has been given in the appendix of Ref. [74].
} 
the Pauli matrices, the generators for the triplet representation are

$$
t^{1}=\frac{1}{\sqrt{2}}\left(\begin{array}{ccc}
0 & 1 & 0 \\
1 & 0 & 1 \\
0 & 1 & 0
\end{array}\right), \quad t^{2}=\frac{1}{\sqrt{2}}\left(\begin{array}{ccc}
0 & -i & 0 \\
i & 0 & -i \\
0 & i & 0
\end{array}\right), \quad t^{3}=\left(\begin{array}{ccc}
1 & 0 & 0 \\
0 & 0 & 0 \\
0 & 0 & -1
\end{array}\right)
$$

and the matrix $U$, which rotates $X$ into the Cartesian basis, is given by [75]

$$
U=\left(\begin{array}{ccc}
-\frac{1}{\sqrt{2}} & 0 & \frac{1}{\sqrt{2}} \\
-\frac{i}{\sqrt{2}} & 0 & -\frac{i}{\sqrt{2}} \\
0 & 1 & 0
\end{array}\right)
$$

Following the same procedure as in the SM, the minimization conditions for the scalar potential read

$$
\begin{aligned}
0=\frac{\partial V}{\partial v_{\phi}}= & v_{\phi}\left[\mu_{2}^{2}+4 \lambda_{1} v_{\phi}^{2}+3\left(2 \lambda_{2}-\lambda_{5}\right) v_{\chi}^{2}-\frac{3}{2} M_{1} v_{\chi}\right] \\
0=\frac{\partial V}{\partial v_{\chi}}= & 3 \mu_{3}^{2} v_{\chi}+3\left(2 \lambda_{2}-\lambda_{5}\right) v_{\phi}^{2} v_{\chi}+12\left(\lambda_{3}+3 \lambda_{4}\right) v_{\chi}^{3}-\frac{3}{4} M_{1} v_{\phi}^{2} \\
& -18 M_{2} v_{\chi}^{2}
\end{aligned}
$$

and can be used to eliminate the $\mu^{2}$ terms as in the SM case.

The Higgs fields can be organized by their representation under the custodial $\mathrm{SU}(2)$ symmetry. Using group theory (or equivalently the addition of spin in quantum mechanics) the doublet is represented by $2 \otimes 2 \rightarrow 3 \oplus 1$ i.e. it produces a triplet and a singlet as in the SM. Similarly, the bitriplet's representation gives: $3 \otimes 3 \rightarrow 5 \oplus 3 \oplus 1$ i.e. a fiveplet, a triplet, and a singlet. One linear combination of the triplets corresponds to the Goldstone bosons meaning the physical fields transform as a fiveplet, a triplet, 
and two singlets The fiveplet and triplet states are given by

$$
\begin{aligned}
& H_{5}^{++}=\chi^{++}, \quad H_{5}^{+}=\frac{\left(\chi^{+}-\xi^{+}\right)}{\sqrt{2}}, \quad H_{5}^{0}=\sqrt{\frac{2}{3}} \xi^{0, r}-\sqrt{\frac{1}{3}} \chi^{0, r}, \\
& H_{3}^{+}=-s_{H} \phi^{+}+c_{H} \frac{\left(\chi^{+}+\xi^{+}\right)}{\sqrt{2}}, \quad H_{3}^{0}=-s_{H} \phi^{0, i}+c_{H} \chi^{0, i},
\end{aligned}
$$

where the vevs are parameterized by

$$
c_{H} \equiv \cos \theta_{H}=\frac{v_{\phi}}{v}, \quad s_{H} \equiv \sin \theta_{H}=\frac{2 \sqrt{2} v_{\chi}}{v}
$$

where $c_{H}^{2}$ and $s_{H}^{2}$ give the fraction of the $\mathrm{W}$ and $\mathrm{Z}$ squared masses generated by the doublet and triplets respectively and we have decomposed the neutral fields into real and imaginary parts according to

$$
\phi^{0} \rightarrow \frac{v_{\phi}}{\sqrt{2}}+\frac{\phi^{0, r}+i \phi^{0, i}}{\sqrt{2}}, \quad \chi^{0} \rightarrow v_{\chi}+\frac{\chi^{0, r}+i \chi^{0, i}}{\sqrt{2}}, \quad \xi^{0} \rightarrow v_{\chi}+\xi^{0, r}
$$

The masses within each custodial multiplet are degenerate at tree level and can be written (after eliminating $\mu_{2}^{2}$ and $\mu_{3}^{2}$ in favour of the vevs) $\mathrm{as}^{3}$

$$
\begin{aligned}
& m_{5}^{2}=\frac{M_{1}}{4 v_{\chi}} v_{\phi}^{2}+12 M_{2} v_{\chi}+\frac{3}{2} \lambda_{5} v_{\phi}^{2}+8 \lambda_{3} v_{\chi}^{2} \\
& m_{3}^{2}=\frac{M_{1}}{4 v_{\chi}}\left(v_{\phi}^{2}+8 v_{\chi}^{2}\right)+\frac{\lambda_{5}}{2}\left(v_{\phi}^{2}+8 v_{\chi}^{2}\right)=\left(\frac{M_{1}}{4 v_{\chi}}+\frac{\lambda_{5}}{2}\right) v^{2} .
\end{aligned}
$$

\footnotetext{
${ }^{3}$ Note that the masses are finite in the limit $v_{\chi} \rightarrow 0$. This is because the ratio $M_{1} / v_{\chi}$ is finite in this limit since the minimization condition $\partial V / \partial v_{\chi}=0$ gives,

$$
\frac{M_{1}}{v_{\chi}}=\frac{4}{v_{\phi}^{2}}\left[\mu_{3}^{2}+\left(2 \lambda_{2}-\lambda_{5}\right) v_{\phi}^{2}+4\left(\lambda_{3}+3 \lambda_{4}\right) v_{\chi}^{2}-6 M_{2} v_{\chi}\right] \text {, }
$$

This implies that $\mathcal{M}_{12}^{2} \rightarrow 0$ (or equivalently that $\sin \alpha \rightarrow 0$ ) when $s_{H} \rightarrow 0$. 
The two custodial SU(2)-singlet mass eigenstates are given by

$$
h=\cos \alpha \phi^{0, r}-\sin \alpha H_{1}^{0 \prime}, \quad H=\sin \alpha \phi^{0, r}+\cos \alpha H_{1}^{0 \prime},
$$

where

$$
H_{1}^{0 \prime}=\sqrt{\frac{1}{3}} \xi^{0, r}+\sqrt{\frac{2}{3}} \chi^{0, r} .
$$

Their mixing angle and masses are given by

$$
\begin{aligned}
& \sin 2 \alpha=\frac{2 \mathcal{M}_{12}^{2}}{m_{H}^{2}-m_{h}^{2}}, \quad \cos 2 \alpha=\frac{\mathcal{M}_{22}^{2}-\mathcal{M}_{11}^{2}}{m_{H}^{2}-m_{h}^{2}} \\
& m_{h, H}^{2}=\frac{1}{2}\left[\mathcal{M}_{11}^{2}+\mathcal{M}_{22}^{2} \mp \sqrt{\left(\mathcal{M}_{11}^{2}-\mathcal{M}_{22}^{2}\right)^{2}+4\left(\mathcal{M}_{12}^{2}\right)^{2}}\right]
\end{aligned}
$$

where we choose $m_{h}<m_{H}$, and

$$
\begin{aligned}
\mathcal{M}_{11}^{2} & =8 \lambda_{1} v_{\phi}^{2} \\
\mathcal{M}_{12}^{2} & =\frac{\sqrt{3}}{2} v_{\phi}\left[-M_{1}+4\left(2 \lambda_{2}-\lambda_{5}\right) v_{\chi}\right] \\
\mathcal{M}_{22}^{2} & =\frac{M_{1} v_{\phi}^{2}}{4 v_{\chi}}-6 M_{2} v_{\chi}+8\left(\lambda_{3}+3 \lambda_{4}\right) v_{\chi}^{2} .
\end{aligned}
$$

The parameters of the custodial-symmetric scalar potential can be constrained theoretically by requiring perturbative unitarity of two-to-two scalar scattering amplitudes and bounded-from-belowness of the scalar potential. Perturbative unitarity requires that the $\lambda_{i}$ obey the following relations $[74,75]$ :

$$
\begin{aligned}
\sqrt{\left(6 \lambda_{1}-7 \lambda_{3}-11 \lambda_{4}\right)^{2}+36 \lambda_{2}^{2}}+\left|6 \lambda_{1}+7 \lambda_{3}+11 \lambda_{4}\right| & <4 \pi \\
\sqrt{\left(2 \lambda_{1}+\lambda_{3}-2 \lambda_{4}\right)^{2}+\lambda_{5}^{2}}+\left|2 \lambda_{1}-\lambda_{3}+2 \lambda_{4}\right| & <4 \pi \\
\left|2 \lambda_{3}+\lambda_{4}\right| & <\pi
\end{aligned}
$$




$$
\left|\lambda_{2}-\lambda_{5}\right|<2 \pi
$$

Requiring that the scalar potential is bounded from below imposes the following constraints [74]:

$$
\begin{aligned}
& \lambda_{1}>0, \\
& \lambda_{4}> \begin{cases}-\frac{1}{3} \lambda_{3} & \text { for } \lambda_{3} \geq 0, \\
-\lambda_{3} & \text { for } \lambda_{3}<0,\end{cases} \\
& \lambda_{2}> \begin{cases}\frac{1}{2} \lambda_{5}-2 \sqrt{\lambda_{1}\left(\frac{1}{3} \lambda_{3}+\lambda_{4}\right)} & \text { for } \lambda_{5} \geq 0 \text { and } \lambda_{3} \geq 0, \\
\omega_{+}(\zeta) \lambda_{5}-2 \sqrt{\lambda_{1}\left(\zeta \lambda_{3}+\lambda_{4}\right)} & \text { for } \lambda_{5} \geq 0 \text { and } \lambda_{3}<0, \\
\omega_{-}(\zeta) \lambda_{5}-2 \sqrt{\lambda_{1}\left(\zeta \lambda_{3}+\lambda_{4}\right)} & \text { for } \lambda_{5}<0,\end{cases}
\end{aligned}
$$

where

$$
\begin{aligned}
\omega_{ \pm}(\zeta) & =\frac{1}{6}(1-B) \pm \frac{\sqrt{2}}{3}\left[(1-B)\left(\frac{1}{2}+B\right)\right]^{1 / 2} \\
B & \equiv \sqrt{\frac{3}{2}\left(\zeta-\frac{1}{3}\right)} \in[0,1] \\
\zeta & \equiv \frac{\operatorname{Tr}\left(X^{\dagger} X X^{\dagger} X\right)}{\left[X^{\dagger} X\right]^{2}}
\end{aligned}
$$

and Eq. (3.21) must be satisfied for all values of $\zeta \in\left[\frac{1}{3}, 1\right]$.

In this thesis, the numerical calculations involving the GM model are performed using the GM model calculator GMCALC [76]. ${ }^{4}$ GMCALC is a FORTRAN program which given a particular parameter set calculates the tree-level mass spectrum, couplings, branching ratios and total widths of the scalar decays. It also can be used to

\footnotetext{
${ }^{4}$ This statement only applies to calculations in the "tree-level" model described in this chapter. Calculations of custodial symmetry violating effects, described in Ch. 4, are done using my own custom-written $\mathrm{C}++$ code.
} 
test whether a parameter point is excluded by applying theoretical and experimental constraints on the input parameter point.

\subsection{Phenomenology}

The GM model provides a prototype for models in which part of electroweak symmetry breaking is due to the vacuum expectation value (vev) of scalars in $\mathrm{SU}(2)_{L}$ representations larger than the doublet ("exotic" scalars). In the GM model, these exotic states are part of the custodial-fiveplet while the remaining scalars are phenomenologically similar to simpler models. For e.g. the phenomenology of the custodial triplet is very similar to the type I $2 \mathrm{HDM}$, where $\cot \theta_{H} \rightarrow \tan \beta(\tan \beta$ being the ratio of the doublets vevs in the $2 \mathrm{HDM}$ ). This makes the phenomenology of the fiveplet the most experimentally compelling aspect of the GM model. Its most important features are: the existence of a doubly charged Higgs, the smoking gun signal of these models; these fiveplet scalars are also fermiophobic, with no coupling to fermions at tree-level; conversely, the $H_{5}^{ \pm} W^{\mp} Z$ coupling is non-zero at tree-level, which distinguishes it from the charged Higgs predicted in the 2HDM. The fiveplet coupling to vector boson pairs is conveniently parameterized by $s_{H}$. Assuming that the branching ratio of the $H_{5}$ to vector bosons is close to 1 , the proportionality of the vector boson fusion production cross section of the $H_{5}$ states (their main production mechanism) to $s_{H}^{2}$ allows LHC searches to directly constrain $s_{H}$ as a function of $m_{5}$ [14-20]. Examples of the constraints from these papers are shown in Fig. 3.1. Not included in this thesis is the recent CMS search [77] which was released after the finalization of our analysis. It is the update of the VBF search shown in the bottom right panel of Fig. 3.1. Below about $m_{5} \sim 500 \mathrm{GeV}$ the new search does not improve on the previous exclusion limit but the exclusion improves above this mass, limiting $s_{H}$ to 

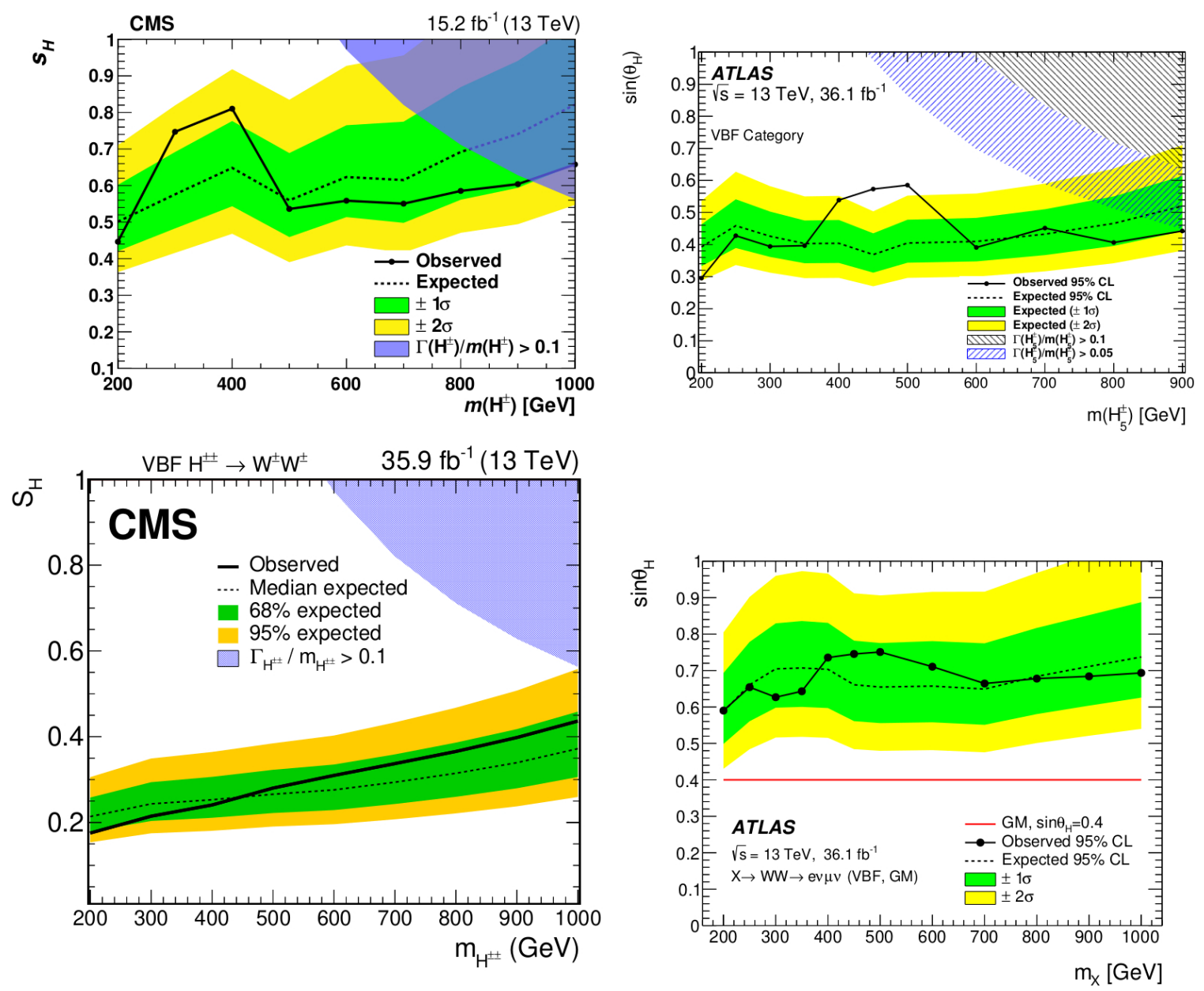

Figure 3.1: Searches for the charged fiveplet states of the GM model at the LHC. Parameter space above the solid black curve is excluded. Top left: CMS search for $H_{5}^{+}$[15] (Fig. 3-b). Top right: ATLAS search for $H_{5}^{+}$[17] (Fig. 7-b). Bottom left: CMS search for $H_{5}^{++}$[16] (Fig. 3-b). An update to this search [77] was published after the finalization of our analysis. See text for a summary of the new limit. Bottom right: ATLAS search for $H_{5}^{++} \rightarrow W W \rightarrow e \nu \mu \nu$ [19] (Fig. 10-b). These plots are reproduced unaltered from the cited references under CC-BY-3.0 [C2015 CERN]. 
lie below $\sim 0.25$ at $m_{5} \sim 700 \mathrm{GeV}$ and $\sim 0.3$ at $m_{5} \sim 1000 \mathrm{GeV}$. It also extends the search to $m_{5}<2000 \mathrm{GeV}$ limiting $s_{H}$ to lie below $\sim 0.35$ at $m_{5} \sim 1500 \mathrm{GeV}$ and $\sim 0.55$ at $m_{5} \sim 2000 \mathrm{GeV}$

To design and interpret these searches, theoretical points are used to seed Monte Carlo event generators which produce simulated signal events. These simulated events allow experimentalists to determine the sensitivity of each point which can then be interpolated between points in the $m_{5}-s_{H}$ plane and compared to data. This process is quite difficult to implement when randomly choosing parameter points as it is easy to choose parameter points which are theoretically excluded, cause the generator to throw an error, or worse simply produce a junk result (e.g. if a coupling is chosen to be non-perturbative, the generated cross-section can become unphysically large); even if events are properly generated, each event would correspond to a different projection onto the $m_{5}-s_{H}$ plane, making it risky to interpolate results across the plane. This process can be greatly simplified if there exists a well defined benchmark plane; where the theoretically allowed and disallowed regions can be separated in the plane and where the parameters are chosen to ensure the allowed points will generate reasonable Monte Carlo events which can be safely interpolated across the benchmark plane. With this in mind, the LHC Higgs Cross Section Working Group developed the H5plane benchmark scenario for the GM model [21,22], defined for $m_{5} \in[200,3000] \mathrm{GeV}$. The H5plane benchmark takes as its two free parameters $m_{5}$ and $s_{H}$ and the other parameters are fixed to ensure $B R\left(H_{5} \rightarrow V V\right)=1$ to a good approximation. These choices are specified in Table 3.1. Its phenomenology was studied in some detail in Ref. [22].

However, for $m_{5}<200 \mathrm{GeV}$, the H5plane benchmark is unable to cover a significant fraction of the region allowed in a general parameter scan. This is not 


\begin{tabular}{lll}
\hline \hline Fixed Parameters & Variable Parameters & Dependent Parameters \\
\hline$G_{F}=1.1663787 \times 10^{-5} \mathrm{GeV}^{-2}$ & $m_{5} \in[200,3000] \mathrm{GeV}$ & $\lambda_{2}=0.4 m_{5} /(1000 \mathrm{GeV})$ \\
$m_{h}=125 \mathrm{GeV}$ & $s_{H} \in(0,1)$ & $M_{1}=\sqrt{2} s_{H}\left(m_{5}^{2}+v^{2}\right) / v$ \\
$\lambda_{3}=-0.1$ & & $M_{2}=M_{1} / 6$ \\
$\lambda_{4}=0.2$ & & \\
\hline \hline
\end{tabular}

Table 3.1: Input parameters for the H5plane benchmark scenario [21] in the custodial-symmetric GM model.

an issue with the current LHC searches for the $H_{5}$ states in the GM model which focus on $H_{5}$ masses of $200 \mathrm{GeV}$ and above (as can be seen in Fig 3.1) for which the decays into vector boson pairs are on shell. Below this mass however, there exists a largely unprobed region in the parameter space with doubly-charged Higgs bosons as light as $120 \mathrm{GeV}$ still allowed. This open region of parameter space could easily be constrained by extending existing experimental searches to lower masses. For example, the ATLAS search for Drell-Yan production of $H^{++} H^{--}$with decays to like-sign $W$ bosons [20], if extended to masses below $200 \mathrm{GeV}$ and using the full Run 2 data-set, would probe this entire neglected low-mass region independent of $s_{H}$ (as the Drell-Yan production cross-section does not depend on $s_{H}$ ) and could potentially entirely exclude $H_{5}$ masses below 200-300 GeV. (The only possible loophole to such an exclusion would be mass spectra engineered to make the Higgs-to-Higgs decays $H_{5}^{++} \rightarrow H_{3}^{+} W^{+}$and/or $H_{5}^{++} \rightarrow H_{3}^{+} H_{3}^{+}$dominate over the $H_{5}^{++} \rightarrow W^{+} W^{+}$decay mode. It is not yet known whether such a scenario can be achieved while satisfying all theoretical and experimental constraints.) To facilitate searches which probe the region of the GM model's parameter space where $m_{5}<200 \mathrm{GeV}$, we propose a new benchmark plane in the GM model, the low- $m_{5}$ benchmark plane, that is valid in the low- $m_{5}$ region, defined in our case to be $m_{5} \in(50,550) \mathrm{GeV}$. This benchmark is described in detail in section 3.3. 


\subsection{Low- $m_{5}$ benchmark}

In order to establish a benchmark plane with only two variable parameters, choices have to be made to fix the five otherwise-free parameters of the GM model. These choices are dictated by the intended purpose of the benchmark, together with convenience of implementation. The low- $m_{5}$ benchmark specifies the nine parameters of the GM model Lagrangian in terms of $m_{h}, G_{F}, m_{5}, s_{H}, \lambda_{2}, \lambda_{3}, \lambda_{4}, \lambda_{5}$, and $M_{2}$ (this set of input parameters is implemented as INPUTSET $=6$ in the GMCALC code for version 1.4 and higher). $m_{h}=125 \mathrm{GeV}$ and $G_{F}$ are fixed to their measured values. The low- $m_{5}$ benchmark is specified in Table 3.2.

The other fixed (or dependent) parameters of the benchmark are chosen to meet the following design considerations:

- The couplings of $h$ are sufficiently close to their SM values to avoid the benchmark being excluded by LHC measurements of $h$ signal strengths. (The signal strength of a process is the rate of the said process normalized to its SM value.) The signal strength which needs controlling is the loop induced decay $h \rightarrow \gamma \gamma$. In the SM at 1-loop this loop induced decay involves diagrams with either a fermion or a gauge boson running in the loop. The addition of the light $H_{5}^{+}$ and $\mathrm{H}_{5}^{++}$scalars running in the loop, in addition to (heavier) $\mathrm{H}_{3}^{+}$in the loop, could significantly modify the induced coupling and potentially exclude parameter points with light $m_{5}$. This possibility is conveniently avoided by the choice $\lambda_{5}=-4 \lambda_{2}$, for which the $h H_{5} H_{5}$ coupling strengths, given by

$$
g_{h 55}=-8 \sqrt{3}\left(\lambda_{3}+\lambda_{4}\right) s_{\alpha} v_{\chi}+\left(4 \lambda_{2}+\lambda_{5}\right) c_{\alpha} v_{\phi}-2 \sqrt{3} M_{2} s_{\alpha},
$$

go to zero in the limit $s_{H} \rightarrow 0$ (in which $s_{\alpha}$ also goes to zero). This strong 
cancellation is not strictly necessary $\left(\lambda_{2}\right.$ can be varied by up to a factor of 2 relative to this choice before Higgs signal strength measurements begin to seriously constrain the parameter space), but it is convenient and does not affect the $H_{5}$ production and decay phenomenology that the benchmark is designed to capture.

- $m_{5}$ is always less than $m_{3}$, to avoid the decays $H_{5} \rightarrow H_{3} V$ or $H_{5} \rightarrow H_{3} H_{3}$. This choice is made to simplify the $H_{5}$ decay phenomenology in the benchmark and to emphasize the importance of the $V V$ decay modes ${ }^{5}$, which are in fact dominant over the majority of the parameter space of the unconstrained GM model. It also avoids exclusion of parts of the benchmark from direct searches for light $H_{3}^{+}$scalars. This mass hierarchy is achieved through the choices of the signs and magnitudes of $M_{2}, \lambda_{3}$, and especially $\lambda_{5}$.

- A sufficiently large portion of the $m_{5}-s_{H}$ plane is allowed by theoretical and indirect experimental constraints within the benchmark for $m_{5}$ values below $200 \mathrm{GeV}$. This is the most challenging consideration to satisfy and dictates the specific choices for the values of $M_{2}, \lambda_{3}, \lambda_{4}$, and $\lambda_{5}$. These choices were largely obtained by trial and error informed by knowledge of the theoretical constraints on the scalar potential.

The remaining underlying Lagrangian parameters are given in terms of the chosen inputs by:

$$
M_{1}=m_{5}^{2} \frac{4 v_{\chi}}{v_{\phi}^{2}}-\frac{3 v_{\chi}^{2}}{v_{\phi}^{2}} M_{2}-6 v_{\chi} \lambda_{5} v_{\chi}^{2}-\frac{32 v_{\chi}^{3}}{v_{\phi}^{2}} \lambda_{3},
$$

\footnotetext{
${ }^{5}$ This choice ensures $\operatorname{BR}\left(H_{5}^{ \pm \pm} \rightarrow W^{ \pm} W^{ \pm}=1\right)$ even below the $W W$ threshold as the competing lepton-number-violating decays are suppressed by the tiny Yukawa coupling needed to generate neutrino masses. We have also neglected decays with additional particles radiated in the final state (e.g. $H_{5}^{ \pm \pm} \rightarrow W^{ \pm} W^{ \pm} \gamma$ ).
} 


\begin{tabular}{lll}
\hline \hline Fixed inputs & Variable parameters & Dependent parameters \\
\hline$G_{F}=1.1663787 \times 10^{-5} \mathrm{GeV}^{-2}$ & $m_{5} \in(50,550) \mathrm{GeV}$ & $\lambda_{2}=0.08\left(m_{5} / 100 \mathrm{GeV}\right)$ \\
$m_{h}=125 \mathrm{GeV}$ & $s_{H} \in(0,1)$ & $\lambda_{5}=-0.32\left(m_{5} / 100 \mathrm{GeV}\right)$ \\
$\lambda_{3}=-1.5$ & & $=-4 \lambda_{2}$ \\
$\lambda_{4}=1.5=-\lambda_{3}$ & & \\
$M_{2}=10 \mathrm{GeV}$ & & \\
\hline \hline
\end{tabular}

Table 3.2: Specification of the low- $m_{5}$ benchmark for the Georgi-Machacek model. This set of input parameters can be accessed in GMCALC [76] by using INPUTSET $=6$.

$$
\begin{aligned}
\mu_{3}^{2} & =m_{5}^{2}-\frac{\lambda_{5}}{2} v_{\phi}^{2}-2 \lambda_{2} v_{\phi}^{2}-12 \lambda_{3} v_{\chi}^{2}-12 \lambda_{4} v_{\chi}^{2}-6 M_{2} v_{\chi} \\
\lambda_{1} & =\frac{1}{8 v_{\phi}^{2}}\left(m_{h}^{2}+\frac{\left[\frac{\sqrt{3}}{2} v_{\phi}\left(-M_{1}+4\left(2 \lambda_{2}-\lambda_{5}\right) v_{\chi}\right)\right]^{2}}{M_{1} \frac{v_{\phi}^{2}}{4 v_{\chi}}-6 M_{2} v_{\chi}+8\left(\lambda_{3}+3 \lambda_{4}\right) v_{\chi}^{2}-m_{h}^{2}}\right) \\
\mu_{2}^{2} & =-4 \lambda_{1} v_{\phi}^{2}-3\left(2 \lambda_{2}-\lambda_{5}\right) v_{\chi}^{2}+\frac{3}{2} M_{1} v_{\chi}
\end{aligned}
$$

\subsubsection{Populated range of the $m_{5}-s_{H}$ plane and existing con- straints}

We now examine the populated range of the $m_{5}-s_{H}$ plane in the low- $m_{5}$ benchmark and compare it to that of a general scan of the parameter space. The results are presented as scatter plots in the $m_{5}-s_{H}$ plane. For all the parameter scans shown here, we impose the theoretical constraints as implemented in GMCALC - i.e., perturbative unitarity of quartic couplings [75], a scalar potential that is bounded from below [74], and the absence of deeper alternative minima [74] (i.e. we require that the minimum is a global minimum). We also impose the indirect constraints, also implemented in GMCALC, from the $S$ parameter (the S parameter is one of the three oblique PeskinTakeuchi parameters which parameterize BSM contributions to electro-weak radiative corrections; i.e. the higher order corrections to the gauge boson propagators) and 
from $B$ physics (coming from the experimental properties of the B mesons; a meson composed of a b quark and a lighter quark), the most stringent in the low- $m_{5}$ region being $B_{s}^{0} \rightarrow \mu^{+} \mu^{-}$(the constraint from $b \rightarrow s \gamma$ is very similar) [78]. We finally examine the direct experimental constraints on the low- $m_{5}$ region, applying direct search constraints, theory recasts and constraints from the codes HiggsBounds 5.3.2 and HiggsSignals 2.2.3 [79], all of which have been implemented in or interfaced to GMCALC 1.5.0 [80]. A similar study of experimental constraints in a parameter scan of the GM model including roughly the same low mass region we are considering was previously carried out in Ref. [81] where they scanned over $m_{5}$ masses up to $1000 \mathrm{GeV}$ with a focus on finding parameter space that allows non-standard decays.

In Fig. 3.2, we show the region of the available parameter space that is accessible in the low- $m_{5}$ benchmark compared to a general scan over the whole parameter space after imposing the theoretical and indirect experimental constraints previously described. The red points correspond to a general scan over the GM parameter space while the black points correspond to a scan over the benchmark. As can be seen, the low- $m_{5}$ benchmark populates a substantial region of parameter space for $m_{5}$ below $200 \mathrm{GeV}$, only failing to cover the full portion of the high $s_{H}$ region. Because the benchmark is optimized to populate the low- $m_{5}$ region, at higher $m_{5}$ it does not cover the full range of $s_{H}$ obtainable in a general scan, and fails the theoretical constraints entirely for $m_{5} \gtrsim 550 \mathrm{GeV}$. Nevertheless, the benchmark provides enough viable parameter space to be used for searches that span $m_{5}$ values from around $60 \mathrm{GeV}$ to of order $500 \mathrm{GeV}$ - in particular, the full range where we expect a Run 2 search for Drell-Yan production of $H_{5}^{++} H_{5}^{--}$to be sensitive.

In Fig. 3.3 we show the benchmark scan and highlight the regions excluded by the various direct experimental constraints as a scatter plot in the $m_{5}-s_{H}$ plane. The 


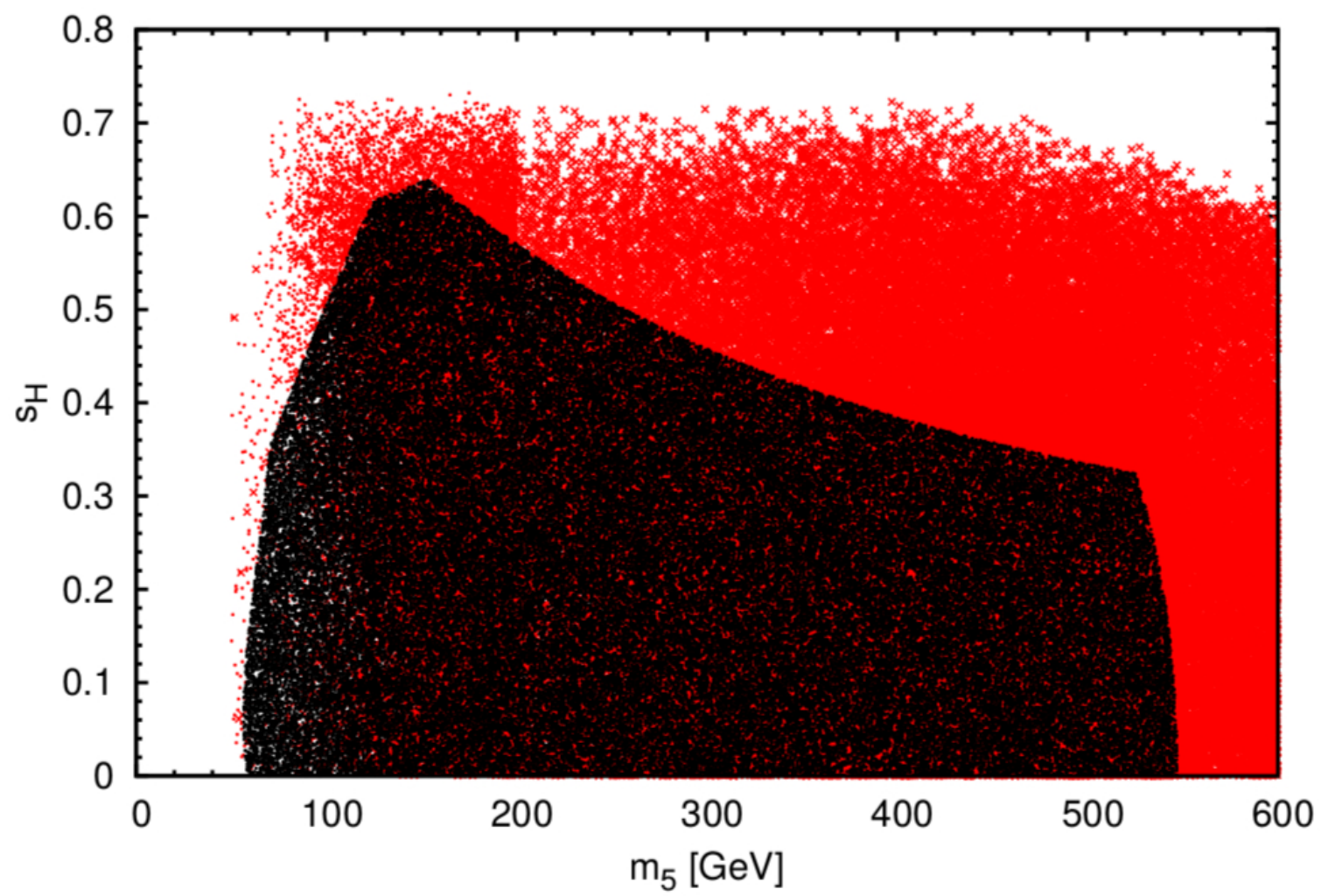

Figure 3.2: The region of $m_{5}$ versus $s_{H}$ that is populated by the low- $m_{5}$ benchmark (black) compared to a general scan over the full parameter space (red), imposing the same theoretical and indirect experimental constraints $\left(S\right.$ parameter and $\left.B_{s} \rightarrow \mu \mu\right)$. Because our general scan is less efficient for small $m_{5}$ values, we include a dedicated general scan restricted to $m_{5}<200 \mathrm{GeV}$ overlaid with a scan for $m_{5}<600 \mathrm{GeV}$ in order to generate a sufficient number of points below $200 \mathrm{GeV}$. 


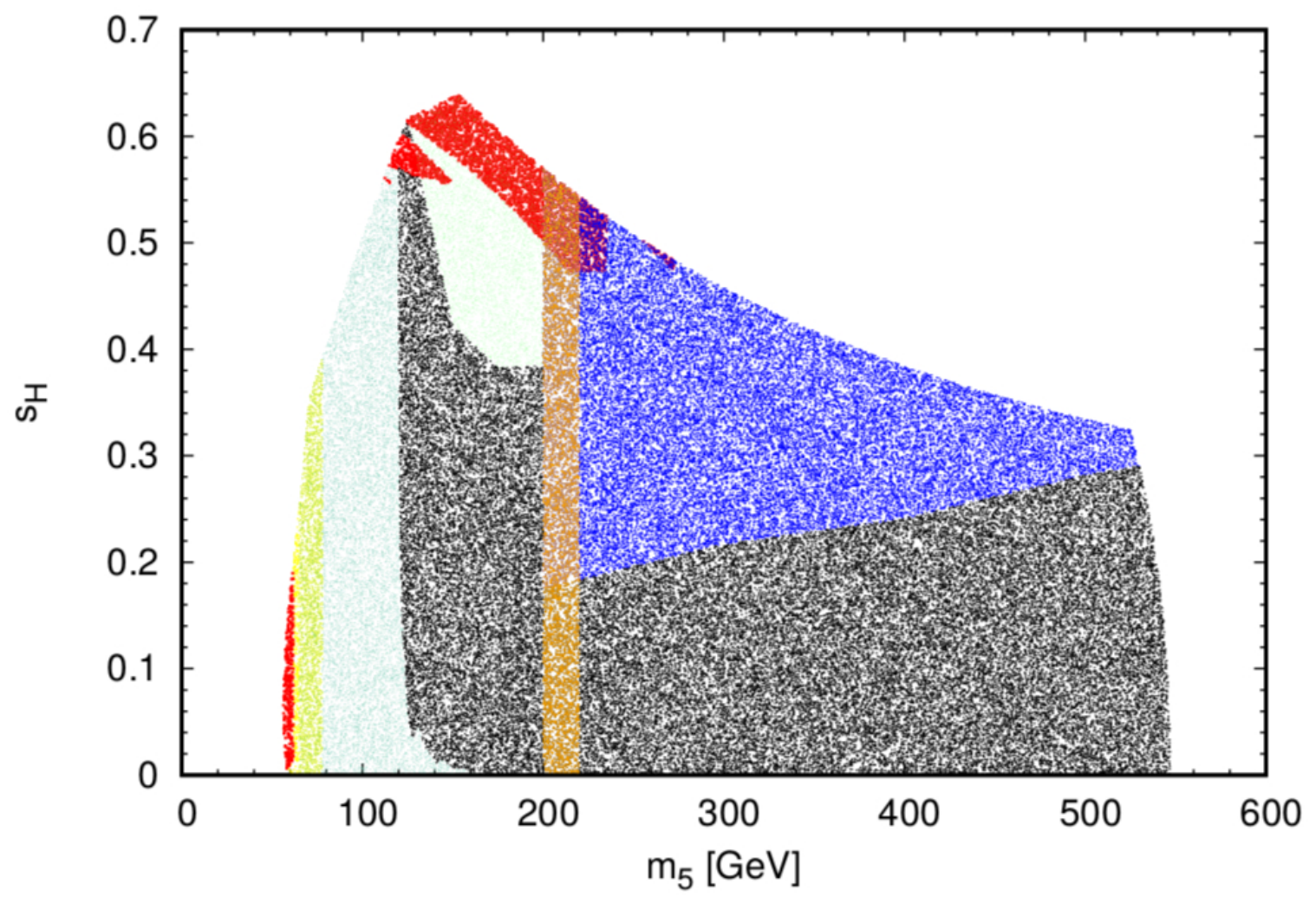

Figure 3.3: The excluded (colored) and allowed (black) points in the scan of the low-mass benchmark shown in Fig. 3.2. The black points are unconstrained, the dark blue points are excluded by the CMS Run 2 direct search for likesign dileptons [16], the orange points are excluded by the ATLAS Run 2 direct search for $H_{5}^{ \pm \pm}$[20] (this exclusion is extended from $m_{5}=220 \mathrm{GeV}$ to $m_{5}=350 \mathrm{GeV}$ when one considers the updated search [84]), the light blue points are excluded by the recast of the ATLAS Run 1 diphoton resonance search [86], the yellow points are excluded by the recast of the ATLAS Run 1 constraint on anomalous like-sign dimuon production [84], the teal points are excluded by the recast of the ATLAS Run $1 \mathrm{VBF} \rightarrow W^{+} W^{+} \rightarrow$ like-sign dileptons cross section measurement [82], and the red points are excluded by searches implemented in HiggsBounds 5.3.2 [79]. 
black points are allowed. The exclusions come from the following sources: a CMS Run 2 search for VBF $H^{ \pm \pm} \rightarrow W^{ \pm} W^{ \pm} \rightarrow$ like-sign dileptons [16], which excludes $s_{H}$ values above $0.2-0.3$ for $m_{5}>200 \mathrm{GeV}$ (dark blue); an ATLAS Run 2 search for DrellYan production of $H^{++} H^{--}$with $H^{ \pm \pm} \rightarrow W^{ \pm} W^{ \pm}$[20] which excludes $200 \mathrm{GeV}<$ $m_{5}<220 \mathrm{GeV}$ (orange) ${ }^{6}$; an ATLAS Run 1 measurement of the $\mathrm{VBF} \rightarrow W^{+} W^{+} \rightarrow$ like-sign dileptons cross section [82] recast by theorists in Ref. [83], which excludes $s_{H}$ values above $0.4-0.6$ for $m_{5}$ between about 120 and $200 \mathrm{GeV}$ (teal); an ATLAS Run 1 search for anomalous like-sign dimuon production [84] recast by theorists in Refs. [85] and [9] to constrain Drell-Yan production of $H_{5}^{ \pm \pm}$, which excludes $m_{5}$ values below $76 \mathrm{GeV}$ independent of the value of $s_{H}$ (yellow); an ATLAS Run 1 diphoton resonance search [86] applied to Drell-Yan production of $H_{5}^{0} H_{5}^{ \pm}$followed by $H_{5}^{0} \rightarrow \gamma \gamma$ which excludes points mainly for $m_{5}$ between 80 and $120 \mathrm{GeV}$ (light blue); and constraints coming from HiggsBounds 5.3.2 [79] (red), which exclude small regions around $s_{H} \sim 0.45-0.65$ and $m_{5} \sim 110-230 \mathrm{GeV}$ from searches for $H \rightarrow Z Z$ and a sliver with $m_{5} \simeq 55-62 \mathrm{GeV}$ and $s_{H} \lesssim 0.2$ from searches for $h \rightarrow H_{5}^{0} H_{5}^{0} \rightarrow 4 \gamma$.

The CMS direct search for VBF $H_{5}^{ \pm \pm} \rightarrow W^{ \pm} W^{ \pm}$is the dominant constraint in the region above $m_{5}=200 \mathrm{GeV}$. The dominant constraints below $m_{5}=200 \mathrm{GeV}$ come from processes involving $H_{5}^{0} \rightarrow \gamma \gamma$. The dominant exclusion is from Drell-Yan production of $H_{5}^{0} H_{5}^{ \pm}$with the $H_{5}^{0}$ decaying to diphotons. Because the Drell-Yan cross section does not depend on $s_{H}$, the excluded region is almost independent of $s_{H}$ (the tail extending to higher $m_{5}$ values at low $s_{H}$ is due to the increase in $\operatorname{BR}\left(H_{5}^{0} \rightarrow \gamma \gamma\right)$ as the competing decays to $W W$ and $Z Z$ are suppressed). This channel excludes $m_{5}$ between 65 and $120 \mathrm{GeV}$ in the low- $m_{5}$ benchmark for any value of $s_{H}$. Although there is a Run 2 version [88] of the Run 1 search for anomalous likesign dimuon

\footnotetext{
${ }^{6}$ An updated ATLAS Run 2 search for Drell-Yan production [87] which was released after the finalization of our analysis extends this limit to $200 \mathrm{GeV}<m_{5}<350 \mathrm{GeV}$.
} 
production which one would expect to compete with the diphoton exclusion, it can not be recast for our purposes because, unlike the Run 1 search, it does not consider invariant masses of the same-sign dileptons below $200 \mathrm{GeV}$. HiggsBounds exclusions from $h \rightarrow H_{5}^{0} H_{5}^{0} \rightarrow 4 \gamma$ also exclude $m_{5}$ between $55-62 \mathrm{GeV}$ for $s_{H} \lesssim 0.2$. The remaining parameter space below $65 \mathrm{GeV}$ is excluded by the Drell-Yan production of $H_{5}^{++} H_{5}^{--}$with decays to like-sign dimuons. The limits at higher $s_{H}$ below $m_{5}=$ $200 \mathrm{GeV}$ are almost entirely due to VBF production of $H_{5}^{ \pm \pm} \rightarrow W^{ \pm} W^{ \pm} \rightarrow$ like-sign dileptons. The HiggsBounds exclusions from production of $H$ decaying to $Z Z$ also exclude a portion of the high $s_{H}, m_{5} \approx 200 \mathrm{GeV}$ region.

We also apply the constraints from the LHC measurements of the $125 \mathrm{GeV}$ Higgs boson signal strength measurements using HiggsSignals 2.2.3 [79]. HiggsSignals performs a p-value test given a specified number of free model parameters. We treat each point as its own model, and hence compute the p-value with zero free parameters. (Varying the number of free parameters in the HigssSignals analysis between 0 and 2 did not have a significant effect on the p-values.) HiggsSignals does not exclude any of the points of the low- $m_{5}$ benchmark; the returned p-values vary between 0.54 and 0.75 , with a p-value of 0.7 over most of the parameter space. This is due in part to our choice of $\lambda_{5}=-4 \lambda_{2}$, which suppresses the tree-level $h H_{5} H_{5}$ couplings that contribute to the loop-induced $h \rightarrow \gamma \gamma$ decay.

In Fig. 3.4 we compare the coverage of the $m_{5}-s_{H}$ plane in the low- $m_{5}$ benchmark with that of the general scan after applying all the experimental constraints listed above. Once again, the red points correspond to a general scan, while the black points correspond to the low- $m_{5}$ benchmark. We can see that the low- $m_{5}$ benchmark can generate points covering the great majority of the allowed region. The survival of general scan points in regions excluded in the benchmark is a result of atypical sup- 


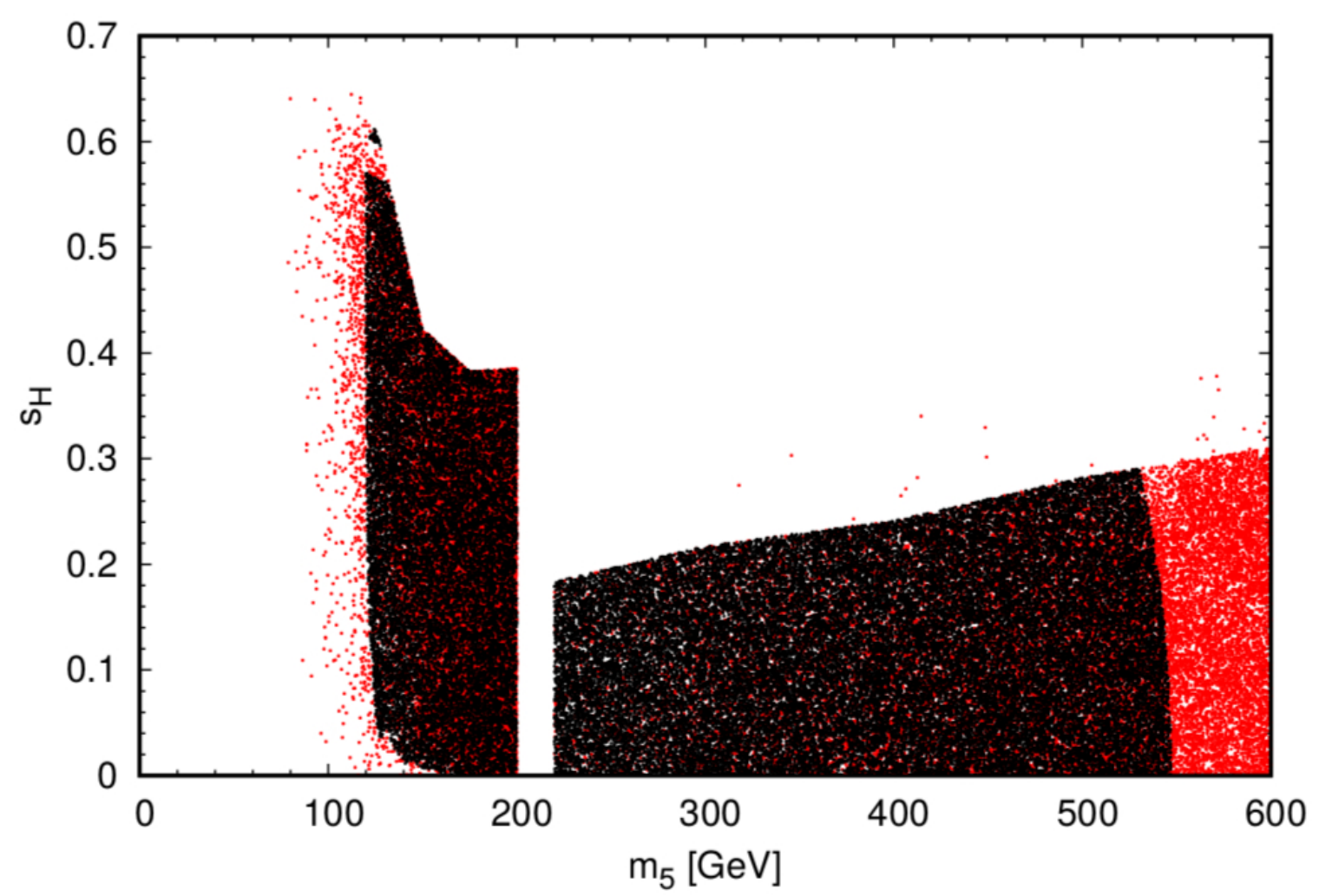

Figure 3.4: The regions of the $m_{5}-s_{H}$ plane populated by the low- $m_{5}$ benchmark (black) and by a general scan (red) after all experimental constraints have been applied. 
pression in the $\mathrm{BR}\left(H_{5}^{++} \rightarrow W^{+} W^{+}\right)$and $\mathrm{BR}\left(H_{5}^{0} \rightarrow \gamma \gamma\right)$ in the high and low $m_{5}$ region respectively. The suppression in $\operatorname{BR}\left(H_{5}^{++} \rightarrow W^{+} W^{+}\right)$is caused by a non-negligible $\mathrm{BR}\left(H_{5}^{++} \rightarrow H_{3}^{+} W^{+}\right)$while the suppression in $\mathrm{BR}\left(H_{5}^{0} \rightarrow \gamma \gamma\right)$ is due to accidental cancellations between the gauge boson and charged scalar loop diagrams. The exclusion of this region in the benchmark is completely dependent on the specific details of the benchmark. It is therefore important that a direct search for $\mathrm{H}_{5}^{++} \mathrm{H}_{5}^{--} \rightarrow 4 l$ probe this region down to the $76 \mathrm{GeV}$ exclusion from the Run 1 direct search. Fortunately, as can be seen in Fig. 3.2, the region where allowed points in the general scan survive the direct constraints is covered by benchmark which can therefore still be used by a direct search to exclude these points.

\subsubsection{Phenomenology of the low- $m_{5}$ benchmark}

In this subsection we examine the properties of the low- $m_{5}$ benchmark. We focus on the phenomenological behaviour of the $H_{5}$ states and the SM-like Higgs, $h$. The results are presented as scatter plots or as contours in the $m_{5}-s_{H}$ plane. As above, for all the parameter scans shown here, we impose the theoretical constraints as implemented in GMCALC [76], the indirect constraints from the $S$ parameter and from $B$ physics, and direct experimental constraints as described in the previous section.

\subsubsection{Couplings of $h$}

The tree-level couplings of the $125 \mathrm{GeV}$ Higgs boson $h$ to fermion pairs and vector boson pairs are expressed in terms of model parameters by

$$
\kappa_{f}^{h}=\frac{c_{\alpha}}{c_{H}}, \quad \quad \kappa_{V}^{h}=c_{\alpha} c_{H}-\sqrt{\frac{8}{3}} s_{\alpha} s_{H},
$$


where each $\kappa$ is defined as the ratio of the corresponding $h$ coupling to that of the SM Higgs boson. These couplings are shown in Figs. 3.5 and 3.6 in the low- $m_{5}$ benchmark, after applying all experimental constraints. Interestingly, in the benchmark both $\kappa_{f}^{h}$ and $\kappa_{V}^{h}$ are always greater than 1, so the SM-like Higgs couplings are always enhanced in the low- $m_{5}$ benchmark as compared to the SM (in a general scan each of these couplings can be either enhanced or suppressed relative to the SM). ${ }^{7}$ The $\kappa_{f}^{h}$ enhancement can be as large as $19 \%$ while the $\kappa_{V}^{h}$ enhancement can be as large as $8 \%$.

In Fig. 3.5 we plot $\kappa_{f}^{h}$ as a function of $m_{5}$ (top) and $s_{H}$ (bottom) in the low- $m_{5}$ benchmark. For $m_{5}<200 \mathrm{GeV}$, a wide range of values are accessible, varying from 1 to a maximum of 1.19 at $m_{5} \simeq 124 \mathrm{GeV}$, with the maximum enhancement falling steadily to 1.06 as $m_{5}$ increases to $200 \mathrm{GeV}$. For $m_{5}>220 \mathrm{GeV}, \kappa_{f}^{h}$ values vary from 1 to about 1.015. These two branches of the benchmark are easily distinguished in the scatter plot versus $s_{H}$ as the two general trends, one a rough parabola corresponding to $m_{5}<200 \mathrm{GeV}$ and the other a linear trend at lower $\kappa_{f}^{h}$ corresponding to $m_{5}>$ $200 \mathrm{GeV}$. The small gap in the allowed values around $\kappa_{f}^{h} \simeq 1.17$ is a result of the small parameter region excluded by searches for $H \rightarrow Z Z$ as implemented in HiggsBounds shown in red in Fig. 3.3.

In Fig. 3.6 we plot $\kappa_{V}^{h}$ as a function of $m_{5}$ (top) and $s_{H}$ (bottom) in the low$m_{5}$ benchmark. For $m_{5}<200 \mathrm{GeV}$, larger enhancements of $\kappa_{V}^{h}$ are possible with values varying from 1 to a maximum of 1.08 at $m_{5} \simeq 124 \mathrm{GeV}$, with the maximum enhancement falling to below 1.04 at $m_{5} \simeq 160 \mathrm{GeV}$ and then rising slightly as $m_{5}$

\footnotetext{
${ }^{7}$ It is worth noting however that this statement applies only to the tree-level couplings. Refs. [89, 90] explored the effects of one-loop corrections to the SM Higgs couplings in the GM model and found a typical suppression of a few percent in the renormalized $\kappa_{f}^{h}$ and $\kappa_{V}^{h}$ couplings compared to their tree-level values. So we expect that at one-loop level it is possible to have $\kappa_{f}^{h}$ and $\kappa_{V}^{h}$ less than 1 in the low- $m_{5}$ benchmark, at least for $s_{H}$ values below about 0.2 for which these couplings only differ from 1 by a few percent.
} 

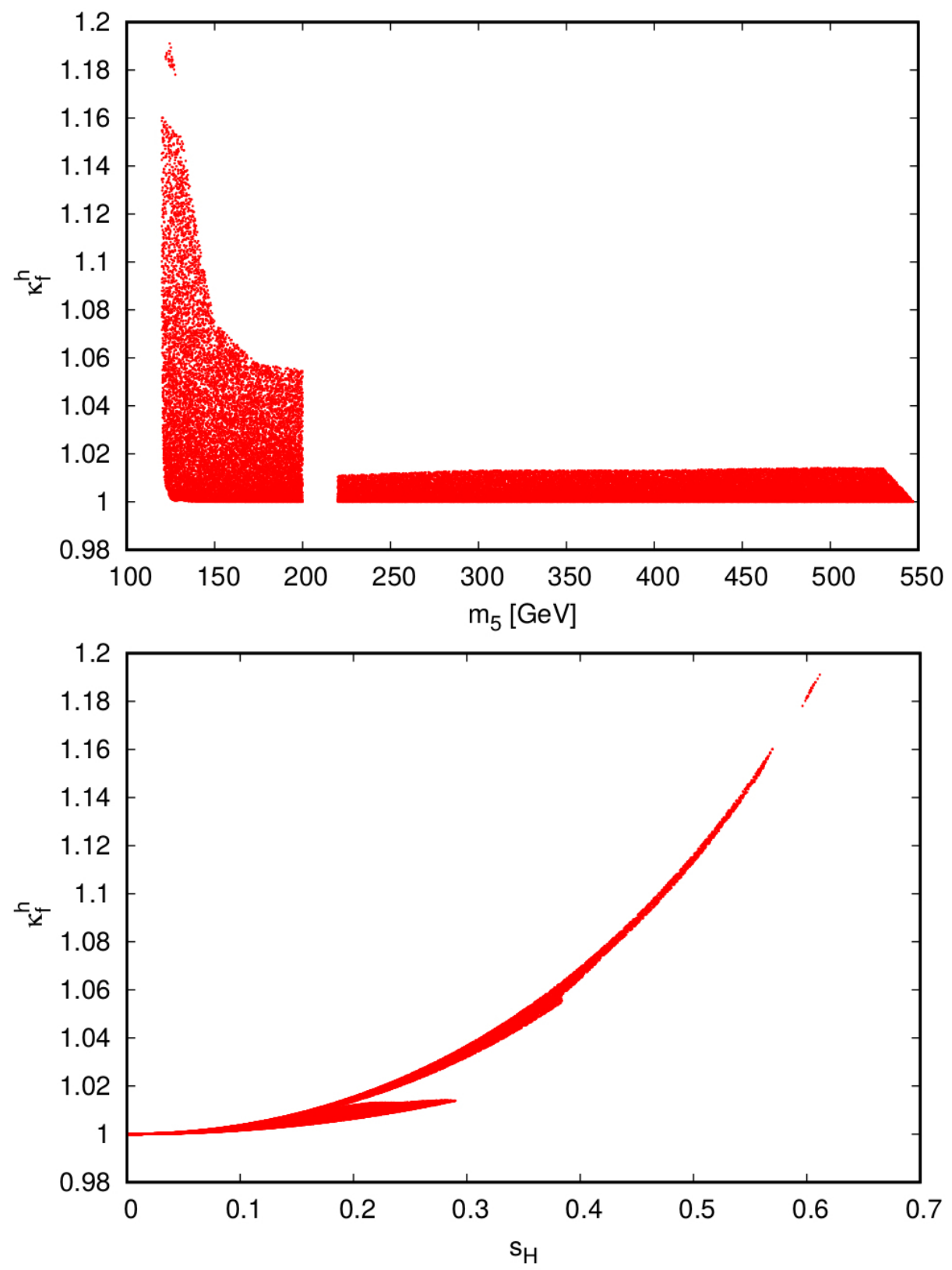

Figure 3.5: Coupling modification factor $\kappa_{f}^{h}$ for the SM-like Higgs $h$ coupling to fermion pairs in the low- $m_{5}$ benchmark, as a function of $m_{5}$ (top) and $s_{H}$ (bottom). The values range between 1 and 1.19. 
increases to $200 \mathrm{GeV}$. For $m_{5}>220 \mathrm{GeV}, \kappa_{V}^{h}$ values vary from 1 to above 1.04 with a maximum value increasing almost linearly with $m_{5}$. In particular, for $m_{5}>220 \mathrm{GeV}$, the allowed enhancements of $\kappa_{V}^{h}$ are substantially larger than those of $\kappa_{f}^{h}$. The two branches of the benchmark are again distinguishable in the scatter plot versus $s_{H}$, with the branch at larger $m_{5}$ appearing at smaller $s_{H}$. Again, the small gap in the allowed values around $\kappa_{V}^{h} \simeq 1.07$ is a result of the small parameter region excluded by searches for $H \rightarrow Z Z$ as implemented in HiggsBounds shown in red in Fig. 3.3. Note that $\kappa_{V}^{h}>1$ at tree level is a distinctive feature of the GM model that cannot occur in extended Higgs sectors containing only scalars in doublets or singlets of $\mathrm{SU}(2)_{L}$.

As LHC measurements of $h$ couplings become more precise we expect that they will begin to constrain the portion of the low- $m_{5}$ benchmark with large $s_{H}$ values; crucially, however, the full range of $m_{5}$ available in the benchmark is consistent with very SM-like couplings of $h$, highlighting the need for direct searches for the $H_{5}$ states.

\subsubsection{Production of $H_{5}$ at the LHC}

We now discuss the major LHC production modes of the $H_{5}$ states in the low- $m_{5}$ benchmark. In Fig. 3.7 we show the $H_{5}$ production cross sections via Drell-Yan production of $H_{5}$ pairs and via VBF. The cross-sections were computed by my collaborator Yongcheng Wu. The Drell-Yan production cross section is independent of $s_{H}$, making it particularly valuable to constrain the GM model at low $s_{H}$. The VBF cross section is proportional to $s_{H}^{2}$; we fix $s_{H}=0.1$ in Fig. 3.7. We computed these cross sections at next-to-leading order (NLO) in QCD for $13 \mathrm{TeV}$ pp collisions using MadGraph5_aMC@NLO [91] with the PDF4LHC15 NLO parton distribution functions [92]. VBF cross sections for production of the $H_{5}$ states at the LHC have previously been computed at next-to-next-to-leading order (NNLO) in QCD for $m_{5} \geq 200 \mathrm{GeV}$ in Ref. [93]. Cross sections for $H_{5}^{ \pm \pm}$production in VBF, associated production with a 

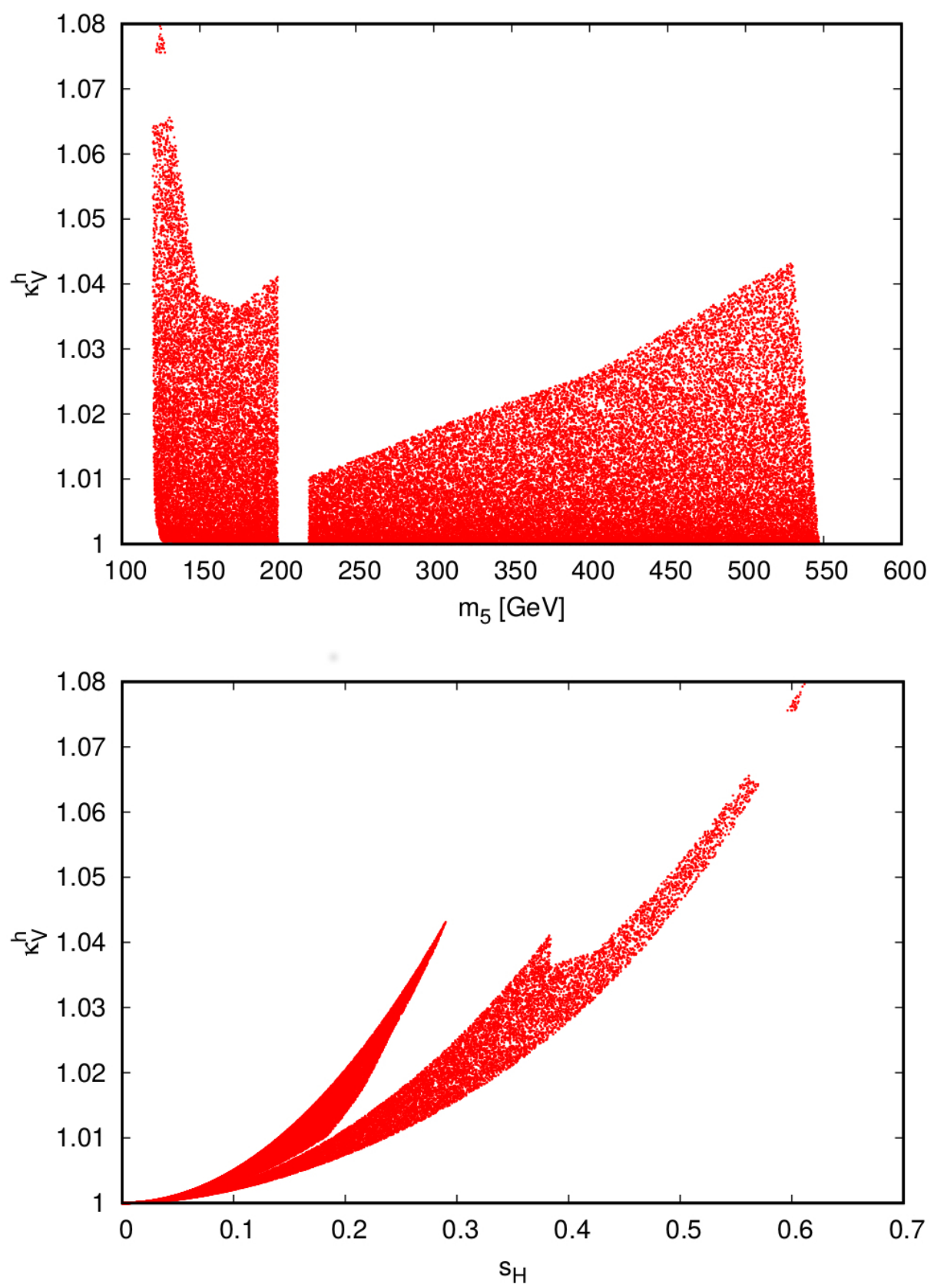

Figure 3.6: Coupling modification factor $\kappa_{V}^{h}$ for the SM-like Higgs $h$ coupling to vector boson pairs in the low- $m_{5}$ benchmark, as a function of $m_{5}$ (top) and $s_{H}$ (bottom). The values range between 1 and 1.08. 

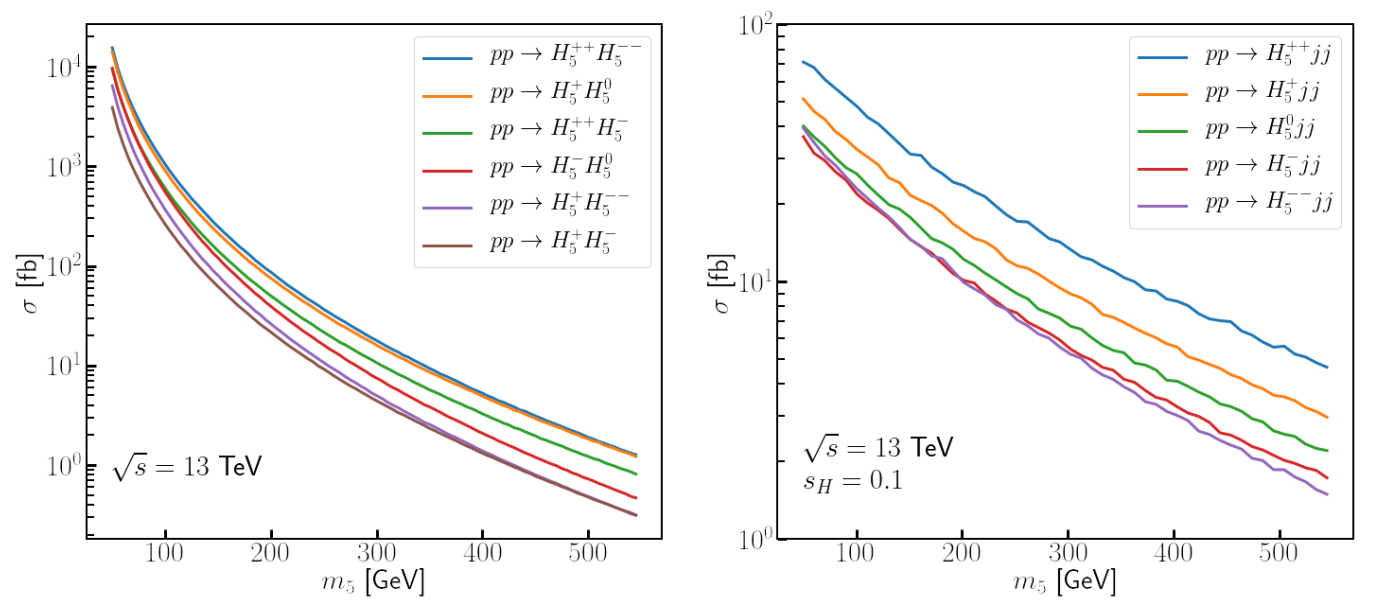

Figure 3.7: Production cross sections for Drell-Yan production of pairs of $H_{5}$ states (left, independent of $s_{H}$ ) and VBF production of single $H_{5}$ states (right, for $s_{H}=0.1$ ) at the $13 \mathrm{TeV}$ LHC, computed at NLO in QCD using MadGraph5_aMC@NLO. This figure was produced by my collaborator Yongcheng $\mathrm{Wu}$ and is used with permisson.

$W$ boson, and Drell-Yan production in pairs were also computed at leading order for $14 \mathrm{TeV}$ and $100 \mathrm{TeV}$ proton-proton collisions in Ref. [81].

The largest Drell-Yan production cross section corresponds to the $H_{5}^{++} H_{5}^{--}$ channel which falls from $545 \mathrm{fb}$ at $m_{5}=120 \mathrm{GeV}$ to $86.3 \mathrm{fb}$ at $m_{5}=200 \mathrm{GeV}$ while the smallest cross section corresponds to the $H_{5}^{+} H_{5}^{-}$channel which falls from $138 \mathrm{fb}$ at $m_{5}=120 \mathrm{GeV}$ to $21.6 \mathrm{fb}$ at $m_{5}=200 \mathrm{GeV}$. The largest VBF production cross section (calculated at $s_{H}=0.1$ ) corresponds to the $H_{5}^{++} j j$ channel which falls from $40.4 \mathrm{fb}$ at $m_{5}=120 \mathrm{GeV}$ to $23.3 \mathrm{fb}$ at $m_{5}=200 \mathrm{GeV}$ while the smallest cross section corresponds to the $H_{5}^{--} j j$ channel which falls from $18.8 \mathrm{fb}$ at $m_{5}=120 \mathrm{GeV}$ to $9.85 \mathrm{fb}$ at $m_{5}=200 \mathrm{GeV}$.

Within the low- $m_{5}$ benchmark, there is a small region of parameter space in which the mass of the heavier custodial-singlet Higgs boson $H$ satisfies $m_{H}>2 m_{5}$, kinematically allowing the decays $H \rightarrow H_{5} H_{5}$. Production of $H$ through, e.g., gluon fusion or VBF followed by decays to $H_{5}$ pairs will therefore enhance the total $H_{5}$ pair production rate in this region of the low- $m_{5}$ benchmark. As is shown below, this 
resonant contribution is always more than an order of magnitude smaller than the cross section from Drell-Yan production. This resonant contribution is very modeldependent, and we advocate that it be omitted when setting bounds on $\mathrm{H}_{5}^{++} \mathrm{H}_{5}^{--}$ production via Drell-Yan in order to allow the limits to be interpreted in as modelindependent a way as possible. We determine the production cross sections of $H$ using the recommended values for beyond-the-SM Higgs bosons from the LHC Higgs Cross Section Working Group [21] together with the appropriate $\kappa_{f}^{H}$ and $\kappa_{V}^{H}$ coupling factors. The expressions for the cross sections are given by

$$
\begin{aligned}
\sigma\left(p p \rightarrow H \rightarrow H_{5}^{++} H_{5}^{--}\right)= & {\left[\left(\kappa_{f}^{H}\right)^{2} \sigma_{G G F}^{H}+\left(\kappa_{V}^{H}\right)^{2}\left(\sigma_{V B F}^{H}+\sigma_{W H}^{H}+\sigma_{Z H}^{H}\right)\right] } \\
& \times B R\left(H \rightarrow H_{5}^{++} H_{5}^{--}\right), \\
\sigma\left(p p \rightarrow H \rightarrow H_{5}^{+} H_{5}^{-}\right)= & {\left[\left(\kappa_{f}^{H}\right)^{2} \sigma_{G G F}^{H}+\left(\kappa_{V}^{H}\right)^{2}\left(\sigma_{V B F}^{H}+\sigma_{W H}^{H}+\sigma_{Z H}^{H}\right)\right] } \\
& \times B R\left(H \rightarrow H_{5}^{+} H_{5}^{-}\right), \\
\sigma\left(p p \rightarrow H \rightarrow H_{5}^{0} H_{5}^{0}\right)= & {\left[\left(\kappa_{f}^{H}\right)^{2} \sigma_{G G F}^{H}+\left(\kappa_{V}^{H}\right)^{2}\left(\sigma_{V B F}^{H}+\sigma_{W H}^{H}+\sigma_{Z H}^{H}\right)\right] } \\
& \times B R\left(H \rightarrow H_{5}^{0} H_{5}^{0}\right),
\end{aligned}
$$

where all cross sections are evaluated at $m_{H}$ (we use linear interpolation between values given in Ref. [21]), $\sigma_{G G F}^{H}$ is the gluon-gluon fusion cross section evaluated at NNLO + next-to-next-to-leading log (NNLL) in QCD (see Ref. [21]), and $\sigma_{V B F}^{H}$, $\sigma_{W H}^{H}$, and $\sigma_{Z H}^{H}$ are the $\mathrm{VBF}, W H$, and $Z H$ associated production cross sections, respectively. Since $B R\left(H \rightarrow H_{5}^{++} H_{5}^{--}\right)=B R\left(H \rightarrow H_{5}^{+} H_{5}^{-}\right)=2 B R\left(H \rightarrow H_{5}^{0} H_{5}^{0}\right)$, the resonant production cross-sections for $p p \rightarrow H \rightarrow H_{5}^{++} H_{5}^{--}$and $p p \rightarrow H \rightarrow$ $H_{5}^{+} H_{5}^{-}$are equal and are twice as large as that of $p p \rightarrow H \rightarrow H_{5}^{0} H_{5}^{0}$.

In Fig. 3.8 we show the region of the $m_{5}-s_{H}$ plane in which $m_{H}>2 m_{5}$ in the low- $m_{5}$ benchmark (gray area and white area with contours above the dotted 
black line). The portion of this region shaded in gray is already excluded by existing searches. In the surviving allowed portion of this region, which spans approximately $120 \mathrm{GeV}<m_{5}<160 \mathrm{GeV}$ and $s_{H}$ between 0.2 and 0.6 , we show contours of the cross section for $H_{5}$ pairs through resonant production of $H$. The cross sections for resonant $H_{5}^{++} H_{5}^{--}$and $H_{5}^{+} H_{5}^{-}\left(H_{5}^{0} H_{5}^{0}\right)$ production have a maximum of $21.5 \mathrm{fb}(10.7 \mathrm{fb})$ at $m_{5} \approx 120 \mathrm{GeV}$, which is more than an order of magnitude smaller than the $545 \mathrm{fb}$ Drell-Yan production cross section of $H_{5}^{++} H_{5}^{--}$at the same mass. It is also roughly an order of magnitude smaller than the individual VBF production cross sections at this mass within the relevant range of $s_{H}$.

\subsubsection{Decays of $\boldsymbol{H}_{5}$}

The low- $m_{5}$ benchmark plane is designed such that $m_{5}<m_{3}$ and $m_{5}<m_{H}$ over the entire benchmark. This means that the branching ratio of $H_{5}^{ \pm \pm} \rightarrow W^{ \pm} W^{ \pm}$is 1 . The decay of the $H_{5}^{ \pm}$is dominated by the $W^{ \pm} Z$ channel for $m_{5}>170 \mathrm{GeV}$ (with a branching ratio of approximately 1) but for masses below this, the loop-induced decays to $W^{ \pm} \gamma$ begin to compete. Similarly the decays of the $H_{5}^{0}$ are dominated by $W^{+} W^{-}$and $Z Z$ above the appropriate thresholds but the loop-induced decays to $\gamma \gamma$ and $Z \gamma$ begin to compete at low $m_{5}$. In this section we show the branching ratios for the most phenomenologically interesting low-mass decays of the $H_{5}^{+}$and $H_{5}^{0}$ to $W^{+} \gamma$ and $\gamma \gamma$ respectively. We compute these branching ratios using GMCALC, which implements doubly-offshell decays of scalars to $W W, Z Z$, and $W Z$ pairs below threshold as well as the loop-induced decays to $\gamma \gamma, Z \gamma$, and $W \gamma$ [94] (decays to $Z \gamma$ and $W \gamma$ with an off-shell $Z$ or $W$ boson are not implemented). Throughout the low- $m_{5}$ benchmark plane, the maximum width-to-mass ratios of the $H_{5}^{0}, H_{5}^{+}$, and $H_{5}^{++}$are $6.4 \times 10^{-3}, 6.5 \times 10^{-3}$ and, $6.6 \times 10^{-3}$ respectively, so the narrow-width approximation is valid throughout the benchmark. 

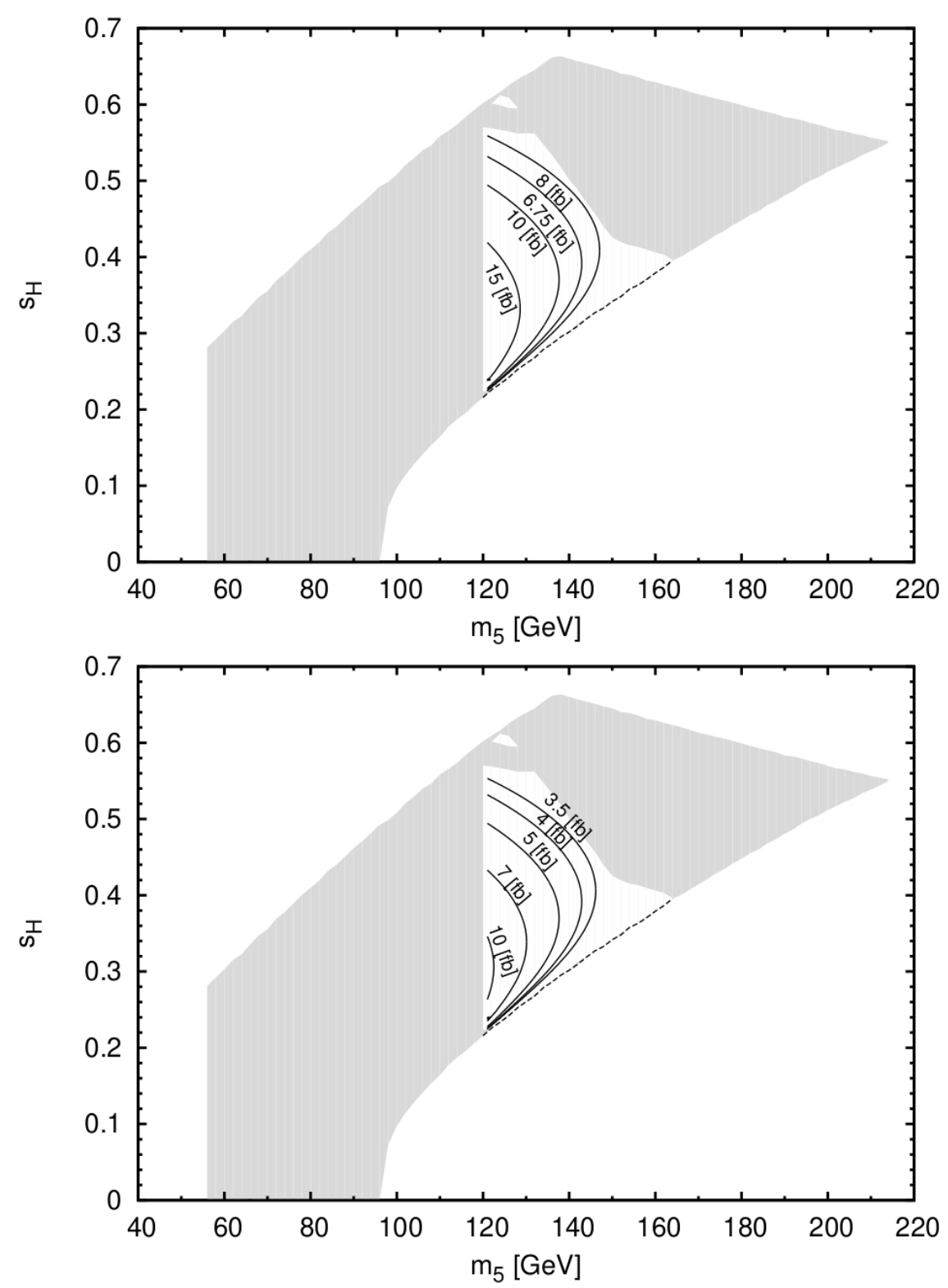

Figure 3.8: Contours of cross sections for the resonant processes $p p \rightarrow H \rightarrow$ $H_{5}^{++} H_{5}^{--}$(equal to $p p \rightarrow H \rightarrow H_{5}^{+} H_{5}^{-}$) (top) and $p p \rightarrow H \rightarrow H_{5}^{0} H_{5}^{0}$ (bottom) in the low- $m_{5}$ benchmark for the $13 \mathrm{TeV}$ LHC. The shaded region has $m_{H}>2 m_{5}$ but is excluded by experimental searches. The region with contours above the dotted black line has $m_{H}>2 m_{5}$ and is still allowed. For $p p \rightarrow H \rightarrow H_{5}^{++} H_{5}^{--}$and $p p \rightarrow H \rightarrow H_{5}^{+} H_{5}^{-}$, the minimum allowed cross section is $0.230 \mathrm{fb}$ and the maximum allowed is $21.5 \mathrm{fb}$. For $p p \rightarrow H \rightarrow H_{5}^{0} H_{5}^{0}$ the minimum allowed cross-section is $0.115 \mathrm{fb}$ and the maximum allowed is $10.7 \mathrm{fb}$. 
In Fig. 3.9 we show the branching ratios of $H_{5}^{0} \rightarrow \gamma \gamma$ (top) and $H_{5}^{+} \rightarrow W^{+} \gamma$ (bottom). The dotted line shows the boundary of the region allowed by experimental constraints; the region below and to the right of the dotted line is allowed (as is a tiny island at $m_{5} \sim 120 \mathrm{GeV}$ and $s_{H} \sim 0.6$ ). To illustrate the impact of the diphoton resonance search on the $H_{5}^{0} \rightarrow \gamma \gamma$ decay, the region excluded by this search is shaded in gray in the right panel of Fig. 3.9. Values of $B R\left(H_{5}^{0} \rightarrow \gamma \gamma\right) \gtrsim 10 \%$ are excluded since the exclusion curve closely follows the 10\% BR contour. Values of $\mathrm{BR}\left(H_{5}^{+} \rightarrow W^{+} \gamma\right)$ as large as $2 \%$ are allowed for small values of $s_{H}$ below 0.05 and $m_{5}$ in the range $125-160 \mathrm{GeV}$.

\subsubsection{Summary}

In this subsection we introduced the low- $m_{5}$ benchmark for the GM model, defined for $m_{5} \in(50,550) \mathrm{GeV}$. The benchmark highlights an unexcluded region of parameter space with light additional Higgs bosons, motivating and facilitating searches for $H_{5}^{++}, H_{5}^{+}$, and $H_{5}^{0}$ at low masses below $200 \mathrm{GeV}$. By design, $m_{3}>m_{5}$ throughout the benchmark plane so that $B R\left(H_{5}^{++} \rightarrow W^{+} W^{+}\right)=1$, while simultaneously ensuring that the modification to $h \rightarrow \gamma \gamma$ by loops of light charged scalars is suppressed. We examined the existing experimental constraints, which limit $s_{H}$ to be strictly below 0.63 in the benchmark, and studied its phenomenology. Our numerical work has been done with a pre-release version of the public code GMCALC 1.5.0.

We showed that the $125 \mathrm{GeV}$ Higgs boson's tree-level couplings to fermion and vector boson pairs are always enhanced in the low- $m_{5}$ benchmark, with enhancements as large as $19 \%$ and $8 \%$, respectively, possible. Nevertheless, the $125 \mathrm{GeV}$ Higgs boson satisfies the Higgs signal strength constraints throughout the entirety of the low- $m_{5}$ benchmark providing further motivation for a direct search for $H_{5}$ states in this region. 

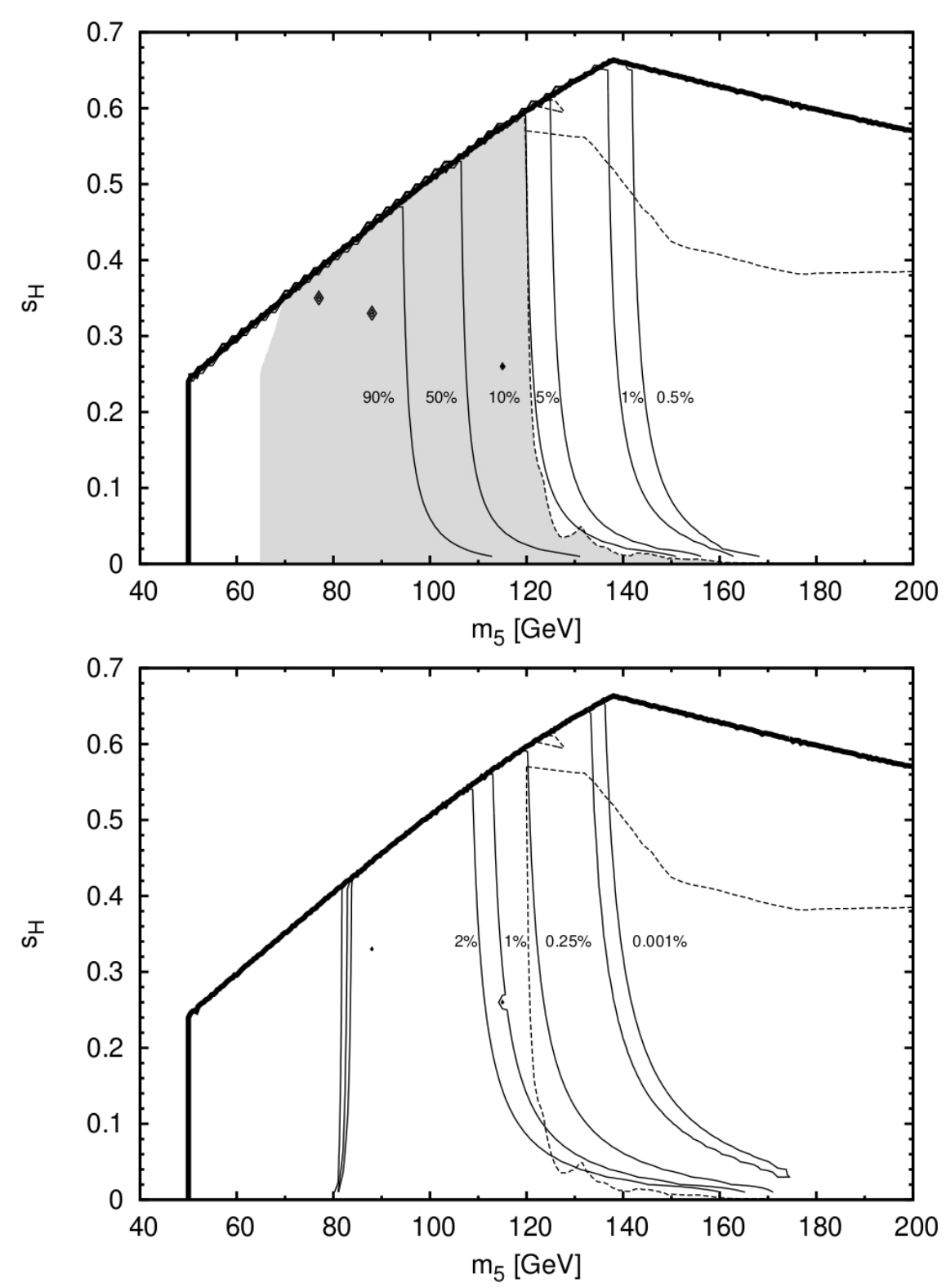

Figure 3.9: Contours of branching ratios of the loop-induced decays of $H_{5}^{0} \rightarrow \gamma \gamma$ (top) and $H_{5}^{+} \rightarrow W^{+} \gamma$ (bottom) in the low- $m_{5}$ benchmark. The region above and to the left of the dotted line is excluded by direct searches. The shaded gray area in the top panel is excluded by diphoton resonance searches applied to $H_{5}^{0} \rightarrow \gamma \gamma$. For $m_{5}<200 \mathrm{GeV}$, the values of $B R\left(H_{5}^{0} \rightarrow \gamma \gamma\right)$ in the low- $m_{5}$ benchmark range between $1.26 \times 10^{-6}$ and 1.00 , surpassing $99 \%$ for $m_{5} \lesssim 80 \mathrm{GeV}$. The values of $B R\left(H_{5}^{+} \rightarrow W^{+} \gamma\right)$ range between zero and 0.887 with the maximum at $m_{5} \simeq 94 \mathrm{GeV}$. 
We also showed that the benchmark-specific enhancement to the $H_{5}$ pair production cross sections by the process $p p \rightarrow H \rightarrow H_{5} H_{5}$ is always at least an order of magnitude smaller than the Drell-Yan production of $H_{5}$ pairs. We anticipate that a dedicated experimental analysis of this Drell-Yan production at the LHC could exclude the entirety of the $m_{5}<200 \mathrm{GeV}$ region. Finally, we showed that $B R\left(H_{5}^{0} \rightarrow \gamma \gamma\right)$ must be less than about $10 \%$ to satisfy constraints from diphoton resonance searches, which in turn forces $B R\left(H_{5}^{+} \rightarrow W \gamma\right)$ to be less than about $2 \%$ in the benchmark. We also pointed out that the width-to-mass ratios of the $H_{5}$ states are below $1 \%$, small enough to justify the narrow width approximation throughout the low- $m_{5}$ benchmark. 


\section{Chapter 4}

\section{Custodial Violation in the GM}

\section{Model}

As the simplest extended Higgs sector which allows for a sizable non-doublet contribution to the masses of the gauge bosons, the GM model is a very useful benchmark model for collider searches. However, it suffers from a problem that has been known since the early '90s; the custodial symmetry in the GM model holds only at tree level [23]. This is a consequence of the gauging of hypercharge which explicitly violates the global $\mathrm{SU}(2)_{R}$ symmetry, leading to an uncontrolled violation of the custodial symmetry at one loop. The most obvious manifestation of this is that the standard calculation of the Peskin-Takeuchi $T$ parameter [95] (closely related to the $\rho$ parameter) yields an infinite result. The problem here is very similar to the case of our toy pseudo-scalar Yukawa theory in Sec. 2.4; to cancel this infinity a counterterm that is absent in the $\mathrm{SU}(2)_{L} \times \mathrm{SU}(2)_{R}$-invariant potential of the GM model but appears in the full gauge-invariant (and custodial-symmetry-violating) theory [23] must be included. The renormalization condition needed to fix the counter-term is equivalent to fixing the value of the $\rho$ parameter; making it an input of the model rather than 
a prediction as is the case for the SM.

A further manifestation of this problem, most relevant for our purposes, is that it is not possible to compute a consistent set of RGEs for the Lagrangian parameters of the custodial-symmetric GM model unless one sets the hypercharge gauge coupling to zero [24]. As an illustration of this problem consider the term $\lambda_{2} \operatorname{Tr}\left(\Phi^{\dagger} \Phi\right) \operatorname{Tr}\left(X^{\dagger} X\right)$ in Eq. 3.7. Expanding out this term gives:

$$
\begin{aligned}
\lambda_{2} \operatorname{Tr}\left(\Phi^{\dagger} \Phi\right) \operatorname{Tr}\left(X^{\dagger} X\right)= & 2 \lambda_{2}\left(\phi^{+} \phi^{-}+\left|\phi^{0}\right|^{2}\right)\left[2\left(\chi^{++} \chi^{--}+\chi^{+} \chi^{-}+\left|\chi^{0}\right|^{2}\right)\right. \\
& \left.+\left(2 \xi^{+} \xi^{-}+\xi^{0}\right)\right] \\
= & 4 \lambda_{2} \phi^{\dagger} \phi \chi^{\dagger} \chi+2 \lambda_{2} \phi^{\dagger} \phi \xi^{\dagger} \xi,
\end{aligned}
$$

where $\phi, \chi$, and $\xi$ are the usual $S U(2)_{L}$ vectors defined in Eq. 4.7 below. As can be seen, this term contains two terms which involve separate interactions of the doublet with the real and complex triplet respectively. In the most general gauge invariant potential, given below in Eq. 4.13, these interactions correspond to two different couplings, $\tilde{\lambda}_{5}$ and $\tilde{\lambda}_{6}$. The requirement of custodial symmetry eliminates one of these free parameters by forcing $\tilde{\lambda}_{5}=4 \lambda_{2}$ and $\tilde{\lambda}_{6}=2 \lambda_{2}$. If custodial symmetry were an exact symmetry of the theory, the RGEs for $\tilde{\lambda}_{5}$ and $\tilde{\lambda}_{6}$ would preserve this relationship and we would be able to write down a RGE for $\lambda_{2}$ by calculating the RGEs using either the $\chi$ terms or the $\xi$ terms. To demonstrate that this is not possible, we can use the formalism of Ref. [45] described in Sec. 2.5 to calculate the hypercharge dependence of the RGEs of $\tilde{\lambda}_{5}$ and $\tilde{\lambda}_{6}$. From Eq. 2.119 we have:

$$
16 \pi^{2} \frac{d f_{i j k l}}{d t}=-12 g^{\prime 2} S_{2}(S) f_{i j k l}+3 g^{\prime 4} A_{i j k l}+\ldots
$$

where for hypercharge interactions $-12 g^{\prime 2} S_{2}(S)=-3 g^{\prime 2}\left[\left(\frac{Y_{i}}{2}\right)+\left(\frac{Y_{j}}{2}\right)+\left(\frac{Y_{k}}{2}\right)+\left(\frac{Y_{l}}{2}\right)\right]$ 
and $3 g^{\prime 4} A_{i j k l}=\frac{3 g^{\prime 4}}{4} Y_{i} Y_{j} Y_{k} Y_{l}\left(\delta_{i j} \delta_{k l}+\delta_{i k} \delta_{j l}+\delta_{i l} \delta_{j k}\right)$. To relate the $f_{i j k l}$ couplings to the $\tilde{\lambda}$ couplings we expand the complex fields (i.e. every field with the exception of $\left.\xi^{0}\right)$ in terms of their real components using $\varphi=\left(\phi_{1}+i \phi_{2}\right) / \sqrt{2}$ and collect terms. This will produce many linearly dependent equations which relate the $f_{i j k l}$ to the $\lambda_{i}$ (or equivalently to the $\tilde{\lambda}_{i}$ ). For the terms we are considering the potential includes:

$$
\begin{aligned}
V & \supset \tilde{\lambda}_{5} \phi^{+} \phi^{-} \chi^{+} \chi^{-}=\frac{\tilde{\lambda}_{5}}{4}\left(\phi_{1}^{2}+\phi_{2}^{2}\right)\left(\phi_{7}^{2}+\phi_{8}^{2}\right) \\
& =\frac{\tilde{\lambda}_{5}}{4} \phi_{1}^{2} \phi_{7}^{2}+\ldots=\frac{\phi_{1}^{2} \phi_{7}^{2}}{4 !}\left(f_{1177}+f_{1771}+f_{7711}+f_{1717}+f_{7117}+f_{7171}\right)+\ldots,
\end{aligned}
$$

where $\phi_{1}=\sqrt{2} \operatorname{Re}\left(\phi^{+}\right), \phi_{2}=\sqrt{2} \operatorname{Im}\left(\phi^{+}\right), \phi_{7}=\sqrt{2} \operatorname{Re}\left(\chi^{+}\right)$, and $\phi_{8}=\sqrt{2} \operatorname{Im}\left(\chi^{+}\right)$. Since the $f_{i j k l}$ are symmetric under the transposition of any two indices, the terms on the right hand side can be written solely in terms of $f_{1177}$. Since this is the only term in the potential which contains $f_{1177}$ we have:

$$
f_{1177}=\frac{4 !}{6} \frac{\tilde{\lambda}_{5}}{4}=\tilde{\lambda}_{5}
$$

The hypercharge contribution to the RGE of $\tilde{\lambda}_{5}$ is therefore:

$$
16 \pi^{2} \frac{d \tilde{\lambda}_{5}}{d t}=16 \pi^{2} \frac{d f_{1177}}{d t}=-3 g^{\prime 2} \frac{5}{2}+3 g^{4}
$$

Expanding the $\tilde{\lambda}_{6}$ term and following similar steps gives $\tilde{\lambda}_{6}=\frac{f_{11 D D}}{2}$, where $D=13$ and $\phi_{13}=\xi^{0}$. The hypercharge contribution to the RGE of $\tilde{\lambda}_{6}$ is therefore:

$$
16 \pi^{2} \frac{d \tilde{\lambda}_{6}}{d t}=\frac{16 \pi^{2}}{2} \frac{d f_{11 D D}}{d t}=-3 g^{\prime 2} \frac{1}{2} \neq 16 \pi^{2} \frac{d\left(\tilde{\lambda}_{5} / 2\right)}{d t} .
$$

As the two RGEs do not agree, we cannot write down a RGE for $\lambda_{2}$ and any relation- 
ship we impose between $\tilde{\lambda}_{5}$ and $\tilde{\lambda}_{6}$ will be broken by the running of the couplings. As this will also apply to the other couplings, it is clear that it is possible to choose the Lagrangian parameters to preserve the custodial symmetry, but only at one energy scale. In order to run away from that special scale, one must use the RGEs computed in the full gauge-invariant but custodial-symmetry-violating potential; the hypercharge contribution then breaks the custodial symmetric relations and causes custodial symmetry violation to build up as one runs. Reference [24] independently studied this effect by assuming that the theory is custodial-symmetric at the weak scale and quantifying the amount of custodial symmetry violation that develops as one runs to higher scales.

In this thesis we take a more general approach. We imagine that the custodialsymmetric GM model arises at some high UV scale, for example as a theory of composite scalars with an accidental global $\mathrm{SU}(2)_{L} \times \mathrm{SU}(2)_{R}$ symmetry in the scalar sector. (Such models have been constructed in the context of little Higgs theories in Refs. [96,97].) Below the compositeness scale, custodial symmetry violation accumulates through the running of the Lagrangian parameters down to the weak scale. Weak-scale measurements of the $\rho$ parameter can then be used to constrain how high the custodial-symmetric scale can be (as we have traded the freedom to fix the $\rho$ parameter in the renormalized theory with the freedom to fix the scale of UV completion). Subject to this constraint, we can also quantify the physical effects of custodial symmetry violation in Higgs-sector observables, such as the ratio of the SM-like Higgs boson couplings to $W W$ and $Z Z$ and custodial-violating mixings and mass splittings among the additional scalars in the GM model. This allows us to answer the main questions which face the GM model; is it simply a toy benchmark model for collider searches or is it a valid effective theory in its own right? Can we discover the 
GM model at the LHC without also discovering its UV completion? And if it is a valid effective theory, do the current experimental constraints placed on the tree-level custodial symmetric theory still apply to the 1-loop custodial symmetry violating theory?

In order to answer these questions it is key that we accurately quantify the custodial symmetry violation allowed in the model for a particular choice of the UV scale. Given the stringent experimental constraints on the $\rho$ parameter, we expect that the amount of custodial symmetry violation should be small and we find it sufficient to work in the leading log approximation-i.e., we use one-loop RGEs and tree-level matching. This is justified by the tiny size of the custodial-violating effects that we find over most of the parameter space. Larger custodial-violating effects arise when scalar masses in the custodial-symmetric theory are tuned to be nearly degenerate, so that custodial symmetry violation induces resonant mixing among mass eigenstates. We handle these situations by exactly diagonalizing the resulting mass matrices; nevertheless, in the small regions of parameter space around these resonances our perturbative calculation remains unstable.

\subsection{Custodial violation and the most general gauge- invariant scalar potential}

In order to allow for custodial symmetry violation, we rewrite the scalar potential in Eq. (3.7) in the most general $\mathrm{SU}(2)_{L} \times \mathrm{U}(1)_{Y}$ gauge invariant form, following Ref. [23]. We can no longer use the bidoublet and bitriplet notation, so we define the scalar 
fields in $\mathrm{SU}(2)_{L}$ vector notation as

$$
\phi=\left(\begin{array}{c}
\phi^{+} \\
\phi^{0}
\end{array}\right), \quad \chi=\left(\begin{array}{c}
\chi^{++} \\
\chi^{+} \\
\chi^{0}
\end{array}\right), \quad \xi=\left(\begin{array}{c}
\xi^{+} \\
\xi^{0} \\
-\xi^{+*}
\end{array}\right)
$$

with vevs given by [compare Eq. (3.13)],

$$
\phi^{0} \rightarrow \frac{\tilde{v}_{\phi}}{\sqrt{2}}+\frac{\phi^{0, r}+i \phi^{0, i}}{\sqrt{2}}, \quad \chi^{0} \rightarrow \tilde{v}_{\chi}+\frac{\chi^{0, r}+i \chi^{0, i}}{\sqrt{2}}, \quad \xi^{0} \rightarrow \tilde{v}_{\xi}+\xi^{0, r}
$$

We use tildes to denote the vevs, parameters, and mass eigenstates of the custodialviolating theory. The vevs of these three fields will be determined by $G_{F}$ according to [compare Eq. (3.6)]

$$
\tilde{v}_{\phi}^{2}+4 \tilde{v}_{\chi}^{2}+4 \tilde{v}_{\xi}^{2} \equiv \tilde{v}^{2}=\frac{1}{\sqrt{2} G_{F}}=v^{2}
$$

and will be constrained by the $\rho$ parameter,

$$
\rho=\frac{\tilde{v}_{\phi}^{2}+4 \tilde{v}_{\chi}^{2}+4 \tilde{v}_{\xi}^{2}}{\tilde{v}_{\phi}^{2}+8 \tilde{v}_{\chi}^{2}}=\frac{v^{2}}{v^{2}+4\left(\tilde{v}_{\chi}^{2}-\tilde{v}_{\xi}^{2}\right)}=1+\Delta \rho
$$

For convenience, we define the conjugate multiplets,

$$
\begin{aligned}
& \tilde{\phi} \equiv C_{2} \phi^{*}=\left(\begin{array}{cc}
0 & 1 \\
-1 & 0
\end{array}\right) \phi^{*}=\left(\begin{array}{c}
\phi^{0 *} \\
-\phi^{+*}
\end{array}\right) \\
& \tilde{\chi} \equiv C_{3} \chi^{*}=\left(\begin{array}{ccc}
0 & 0 & 1 \\
0 & -1 & 0 \\
1 & 0 & 0
\end{array}\right) \chi^{*}=\left(\begin{array}{c}
\chi^{0 *} \\
-\chi^{+*} \\
\chi^{++*}
\end{array}\right) .
\end{aligned}
$$


We also define the following matrix forms of the triplet fields,

$$
\begin{aligned}
& \Delta_{2} \equiv \sqrt{2} \tau^{a} U_{a i} \chi_{i}=\left(\begin{array}{cc}
\chi^{+} / \sqrt{2} & -\chi^{++} \\
\chi^{0} & -\chi^{+} / \sqrt{2}
\end{array}\right), \\
& \Delta_{0} \equiv \sqrt{2} \tau^{a} U_{a i} \xi_{i}=\left(\begin{array}{cc}
\xi^{0} / \sqrt{2} & -\xi^{+} \\
-\xi^{+*} & -\xi^{0} / \sqrt{2}
\end{array}\right), \\
& \bar{\Delta}_{0} \equiv-t^{a} U_{a i} \xi_{i}=\left(\begin{array}{ccc}
-\xi^{0} & \xi^{+} & 0 \\
\xi^{+*} & 0 & \xi^{+} \\
0 & \xi^{+*} & \xi^{0}
\end{array}\right) .
\end{aligned}
$$

The most general gauge invariant scalar potential can then be written as

$$
\begin{aligned}
V(\phi, \chi, \xi)= & \tilde{\mu}_{2}^{2} \phi^{\dagger} \phi+\tilde{\mu}_{3}^{\prime 2} \chi^{\dagger} \chi+\frac{\tilde{\mu}_{3}^{2}}{2} \xi^{\dagger} \xi+\tilde{\lambda}_{1}\left(\phi^{\dagger} \phi\right)^{2}+\tilde{\lambda}_{2}\left|\tilde{\chi}^{\dagger} \chi\right|^{2}+\tilde{\lambda}_{3}\left(\phi^{\dagger} \tau^{a} \phi\right)\left(\chi^{\dagger} t^{a} \chi\right) \\
& +\left[\tilde{\lambda}_{4}\left(\tilde{\phi}^{\dagger} \tau^{a} \phi\right)\left(\chi^{\dagger} t^{a} \xi\right)+\text { h.c. }\right]+\tilde{\lambda}_{5}\left(\phi^{\dagger} \phi\right)\left(\chi^{\dagger} \chi\right)+\tilde{\lambda}_{6}\left(\phi^{\dagger} \phi\right)\left(\xi^{\dagger} \xi\right) \\
& +\tilde{\lambda}_{7}\left(\chi^{\dagger} \chi\right)^{2}+\tilde{\lambda}_{8}\left(\xi^{\dagger} \xi\right)^{2}+\tilde{\lambda}_{9}\left|\chi^{\dagger} \xi\right|^{2}+\tilde{\lambda}_{10}\left(\chi^{\dagger} \chi\right)\left(\xi^{\dagger} \xi\right) \\
& -\frac{1}{2}\left[\tilde{M}_{1}^{\prime} \phi^{\dagger} \Delta_{2} \tilde{\phi}+\text { h.c. }\right]+\frac{\tilde{M}_{1}}{\sqrt{2}} \phi^{\dagger} \Delta_{0} \phi-6 \tilde{M}_{2} \chi^{\dagger} \bar{\Delta}_{0} \chi
\end{aligned}
$$

Note that $\tilde{\lambda}_{4}$ and $\tilde{M}_{1}^{\prime}$ are complex in general, while the rest of the parameters are real. We have adopted the same notation as in Eq. (3.2) of Ref. [23] for the coefficients of the quartic terms, and we have added the trilinear terms that were eliminated in Ref. [23] by the imposition of a $Z_{2}$ symmetry. This scalar potential has also been written down (for real $\tilde{\lambda}_{4}$ and $\tilde{M}_{1}^{\prime}$ ) in Ref. [24]; we give a translation table to their notation in Appendix A.

We note that the last term in Eq. (4.13) can also be written as

$$
-6 \tilde{M}_{2} \chi^{\dagger} \bar{\Delta}_{0} \chi=-6 \tilde{M}_{2} \epsilon_{i j k} \tilde{\chi}_{i} \xi_{j} \chi_{k}
$$


where $\epsilon_{i j k}$ is the totally antisymmetric tensor with $\epsilon_{123}=+1$.

In the custodially-symmetric limit, the Lagrangian parameters in the gaugeinvariant scalar potential in Eq. (4.13) reduce to those in the custodially-symmetric potential in Eq. (3.7) according to

$$
\begin{aligned}
\tilde{\mu}_{2}^{2} & =\mu_{2}^{2} \\
\tilde{\mu}_{3}^{\prime 2} & =\mu_{3}^{2} \\
\tilde{\mu}_{3}^{2} & =\mu_{3}^{2} \\
\tilde{\lambda}_{1} & =4 \lambda_{1} \\
\tilde{\lambda}_{2} & =2 \lambda_{3} \\
\tilde{\lambda}_{3} & =-2 \lambda_{5} \\
\tilde{\lambda}_{4} & =-\sqrt{2} \lambda_{5} \\
\tilde{\lambda}_{5} & =4 \lambda_{2} \\
\tilde{\lambda}_{6} & =2 \lambda_{2} \\
\tilde{\lambda}_{7} & =2 \lambda_{3}+4 \lambda_{4} \\
\tilde{\lambda}_{8} & =\lambda_{3}+\lambda_{4} \\
\tilde{\lambda}_{9} & =4 \lambda_{3} \\
\tilde{\lambda}_{10} & =4 \lambda_{4} \\
\tilde{M}_{1}^{\prime} & =M_{1} \\
\tilde{M}_{1} & =M_{1} \\
\tilde{M}_{2} & =M_{2} \\
&
\end{aligned}
$$

where the relations among the quadratic and quartic couplings are in agreement with Ref. [23]. 
Replacing the fields with their vevs and assuming CP conservation, the most general scalar potential becomes

$$
\begin{aligned}
V\left(v_{\phi}, v_{\chi}, v_{\xi}\right)= & \frac{\tilde{\mu}_{2}^{2}}{2} \tilde{v}_{\phi}^{2}+\tilde{\mu}_{3}^{\prime 2} \tilde{v}_{\chi}^{2}+\frac{\tilde{\mu}_{3}^{2}}{2} \tilde{v}_{\xi}^{2} \\
& +\frac{\tilde{\lambda}_{1}}{4} \tilde{v}_{\phi}^{4}+\frac{\tilde{\lambda}_{3}}{4} \tilde{v}_{\phi}^{2} \tilde{v}_{\chi}^{2}+\frac{\tilde{\lambda}_{4}}{\sqrt{2}} \tilde{v}_{\phi}^{2} \tilde{v}_{\chi} \tilde{v}_{\xi} \\
& +\frac{\tilde{\lambda}_{5}}{2} \tilde{v}_{\phi}^{2} \tilde{v}_{\chi}^{2}+\frac{\tilde{\lambda}_{6}}{2} \tilde{v}_{\phi}^{2} \tilde{v}_{\xi}^{2}+\tilde{\lambda}_{7} \tilde{v}_{\chi}^{4}+\tilde{\lambda}_{8} \tilde{v}_{\xi}^{4}+\tilde{\lambda}_{10} \tilde{v}_{\chi}^{2} \tilde{v}_{\xi}^{2} \\
& -\frac{\tilde{M}_{1}^{\prime}}{2} \tilde{v}_{\phi}^{2} \tilde{v}_{\chi}-\frac{\tilde{M}_{1}}{4} \tilde{v}_{\phi}^{2} \tilde{v}_{\xi}-6 \tilde{M}_{2} \tilde{v}_{\chi}^{2} \tilde{v}_{\xi} .
\end{aligned}
$$

Minimizing this potential yields three equations:

$$
\begin{aligned}
& 0=\frac{\partial V}{\partial \tilde{v}_{\phi}}=\tilde{v}_{\phi}\left[\tilde{\mu}_{2}^{2}+\tilde{\lambda}_{1} \tilde{v}_{\phi}^{2}+\frac{\tilde{\lambda}_{3}}{2} \tilde{v}_{\chi}^{2}+\sqrt{2} \tilde{\lambda}_{4} \tilde{v}_{\chi} \tilde{v}_{\xi}+\tilde{\lambda}_{5} \tilde{v}_{\chi}^{2}+\tilde{\lambda}_{6} \tilde{v}_{\xi}^{2}-\tilde{M}_{1}^{\prime} \tilde{v}_{\chi}\right. \\
& \left.-\frac{\tilde{M}_{1}}{2} \tilde{v}_{\xi}\right] \\
& 0=\frac{\partial V}{\partial \tilde{v}_{\chi}}=2 \tilde{\mu}_{3}^{\prime 2} \tilde{v}_{\chi}+\frac{\tilde{\lambda}_{3}}{2} \tilde{v}_{\phi}^{2} \tilde{v}_{\chi}+\frac{\tilde{\lambda}_{4}}{\sqrt{2}} \tilde{v}_{\phi}^{2} \tilde{v}_{\xi}+\tilde{\lambda}_{5} \tilde{v}_{\phi}^{2} \tilde{v}_{\chi}+4 \tilde{\lambda}_{7} \tilde{v}_{\chi}^{3}+2 \tilde{\lambda}_{10} \tilde{v}_{\chi} \tilde{v}_{\xi}^{2} \\
& -\frac{\tilde{M}_{1}^{\prime}}{2} \tilde{v}_{\phi}^{2}-12 \tilde{M}_{2} \tilde{v}_{\chi} \tilde{v}_{\xi} \\
& 0=\frac{\partial V}{\partial \tilde{v}_{\xi}}=\tilde{\mu}_{3}^{2} \tilde{v}_{\xi}+\frac{\tilde{\lambda}_{4}}{\sqrt{2}} \tilde{v}_{\phi}^{2} \tilde{v}_{\chi}+\tilde{\lambda}_{6} \tilde{v}_{\phi}^{2} \tilde{v}_{\xi}+4 \tilde{\lambda}_{8} \tilde{v}_{\xi}^{3}+2 \tilde{\lambda}_{10} \tilde{v}_{\chi}^{2} \tilde{v}_{\xi}-\frac{\tilde{M}_{1}}{4} \tilde{v}_{\phi}^{2} \\
& -6 \tilde{M}_{2} \tilde{v}_{\chi}^{2}
\end{aligned}
$$

When the $\mathrm{SU}(2)_{L} \times \mathrm{SU}(2)_{R}$ symmetry is imposed, these conditions reduce to those in Eq. (3.10).

The one-loop RGEs for the parameters of the most general gauge invariant potential were calculated by my collaborator Terry Pilkington. They are given in Appendix A for completeness. 


\subsection{Physical masses and mixing in the custodial symmetry violating theory}

Isolating all terms quadratic in scalar fields from the potential and using Eqs. (4.17$4.19)$ to eliminate $\tilde{\mu}_{2}^{2}, \tilde{\mu}_{3}^{\prime 2}$ and $\tilde{\mu}_{3}^{2}$ in favour of the vevs yields the following mass matrices for the physical scalars.

There is only one doubly-charged scalar, $\tilde{H}_{5}^{++}=\chi^{++}=H_{5}^{++}$, and its mass is given by

$$
m_{\tilde{H}_{5}^{++}}^{2}=4 \tilde{\lambda}_{2} \tilde{v}_{\chi}^{2}-\frac{\tilde{\lambda}_{3} \tilde{v}_{\phi}^{2}}{2}-\frac{\tilde{\lambda}_{4} \tilde{v}_{\phi}^{2} \tilde{v}_{\xi}}{2 \sqrt{2} \tilde{v}_{\chi}}+\frac{\tilde{M}_{1}^{\prime}}{4 \tilde{v}_{\chi}} \tilde{v}_{\phi}^{2}+12 \tilde{M}_{2} \tilde{v}_{\xi}
$$

There are two CP-odd neutral scalars (one of which becomes the neutral Goldstone boson), whose mass-squared matrix in the basis $\left(\chi^{0, i}, \phi^{0, i}\right)$ is given by

$$
\mathcal{M}_{i}^{2}=\left(\begin{array}{cc}
\mathcal{M}_{i, 11}^{2} & \mathcal{M}_{i, 12}^{2} \\
\mathcal{M}_{i, 12}^{2} & \mathcal{M}_{i, 22}^{2}
\end{array}\right)
$$

where

$$
\begin{aligned}
\mathcal{M}_{i, 11}^{2} & =-\frac{\tilde{\lambda}_{4} \tilde{v}_{\phi}^{2} \tilde{v}_{\xi}}{2 \sqrt{2} \tilde{v}_{\chi}}+\frac{\tilde{M}_{1}^{\prime}}{4 \tilde{v}_{\chi}} \tilde{v}_{\phi}^{2} \\
\mathcal{M}_{i, 22}^{2} & =-2 \sqrt{2} \tilde{\lambda}_{4} \tilde{v}_{\chi} \tilde{v}_{\xi}+2 \tilde{M}_{1}^{\prime} \tilde{v}_{\chi} \\
\mathcal{M}_{i, 12}^{2} & =\tilde{\lambda}_{4} \tilde{v}_{\phi} \tilde{v}_{\xi}-\frac{\tilde{M}_{1}^{\prime}}{\sqrt{2}} \tilde{v}_{\phi} .
\end{aligned}
$$

Note that the mass-squared matrix for the neutral imaginary states can be written as

$$
\mathcal{M}_{i}^{2}=\left[\frac{\tilde{M}_{1}^{\prime}}{4 \tilde{v}_{\chi}}-\frac{\tilde{\lambda}_{4} \tilde{v}_{\xi}}{2 \sqrt{2} \tilde{v}_{\chi}}\right]\left(\begin{array}{cc}
\tilde{v}_{\phi}^{2} & -\sqrt{8} \tilde{v}_{\phi} \tilde{v}_{\chi} \\
-\sqrt{8} \tilde{v}_{\phi} \tilde{v}_{\chi} & 8 \tilde{v}_{\chi}^{2}
\end{array}\right)
$$


This matrix is easily diagonalized, yielding exact mass eigenstates

$$
\tilde{G}^{0}=\frac{\tilde{v}_{\phi} \phi^{0, i}+\sqrt{8} \tilde{v}_{\chi} \chi^{0, i}}{\sqrt{\tilde{v}_{\phi}^{2}+8 \tilde{v}_{\chi}^{2}}}, \quad \quad \tilde{H}_{3}^{0}=\frac{-\sqrt{8} \tilde{v}_{\chi} \phi^{0, i}+\tilde{v}_{\phi} \chi^{0, i}}{\sqrt{\tilde{v}_{\phi}^{2}+8 \tilde{v}_{\chi}^{2}}}
$$

where $\tilde{G}^{0}$ is the (massless) neutral Goldstone boson and the mass of $\tilde{H}_{3}^{0}$ is given by

$$
m_{\tilde{H}_{3}^{0}}^{2}=\left[\frac{\tilde{M}_{1}^{\prime}}{4 \tilde{v}_{\chi}}-\frac{\tilde{\lambda}_{4} \tilde{v}_{\xi}}{2 \sqrt{2} \tilde{v}_{\chi}}\right]\left(\tilde{v}_{\phi}^{2}+8 \tilde{v}_{\chi}^{2}\right)
$$

There are three singly-charged scalars (one of which becomes the charged Goldstone boson), whose mass-squared matrix in the basis $\left(\chi^{+}, \xi^{+}, \phi^{+}\right)$is given by

$$
\mathcal{M}_{+}^{2}=\left(\begin{array}{ccc}
\mathcal{M}_{+, 11}^{2} & \mathcal{M}_{+, 12}^{2} & \mathcal{M}_{+, 13}^{2} \\
\mathcal{M}_{+, 12}^{2} & \mathcal{M}_{+, 22}^{2} & \mathcal{M}_{+, 23}^{2} \\
\mathcal{M}_{+, 13}^{2} & \mathcal{M}_{+, 23}^{2} & \mathcal{M}_{+, 33}^{2}
\end{array}\right)
$$

where

$$
\begin{aligned}
\mathcal{M}_{+, 11}^{2} & =-\frac{\tilde{\lambda}_{3} \tilde{v}_{\phi}^{2}}{4}-\frac{\tilde{\lambda}_{4} \tilde{v}_{\phi}^{2} \tilde{v}_{\xi}}{2 \sqrt{2} \tilde{v}_{\chi}}+\tilde{\lambda}_{9} \tilde{v}_{\xi}^{2}+\frac{\tilde{M}_{1}^{\prime}}{4 \tilde{v}_{\chi}} \tilde{v}_{\phi}^{2}+6 \tilde{M}_{2} \tilde{v}_{\xi}, \\
\mathcal{M}_{+, 22}^{2} & =-\frac{\tilde{\lambda}_{4} \tilde{v}_{\phi}^{2} \tilde{v}_{\chi}}{\sqrt{2} \tilde{v}_{\xi}}+\tilde{\lambda}_{9} \tilde{v}_{\chi}^{2}+\frac{\tilde{M}_{1}}{4 \tilde{v}_{\xi}} \tilde{v}_{\phi}^{2}+6 \tilde{M}_{2} \frac{\tilde{v}_{\chi}^{2}}{\tilde{v}_{\xi}} \\
\mathcal{M}_{+, 33}^{2} & =-\tilde{\lambda}_{3} \tilde{v}_{\chi}^{2}-\sqrt{2} \tilde{\lambda}_{4} \tilde{v}_{\chi} \tilde{v}_{\xi}+\tilde{M}_{1} \tilde{v}_{\xi}+\tilde{M}_{1}^{\prime} \tilde{v}_{\chi}, \\
\mathcal{M}_{+, 12}^{2}= & \frac{\tilde{\lambda}_{4} \tilde{v}_{\phi}^{2}}{2 \sqrt{2}}-\tilde{\lambda}_{9} \tilde{v}_{\chi} \tilde{v}_{\xi}-6 \tilde{M}_{2} \tilde{v}_{\chi}, \\
\mathcal{M}_{+, 13}^{2}= & \frac{\tilde{\lambda}_{3} \tilde{v}_{\phi} \tilde{v}_{\chi}}{2}-\frac{\tilde{M}_{1}^{\prime}}{2} \tilde{v}_{\phi}, \\
\mathcal{M}_{+, 23}^{2} & =\frac{\tilde{\lambda}_{4} \tilde{v}_{\phi} \tilde{v}_{\chi}}{\sqrt{2}}-\frac{\tilde{M}_{1}}{2} \tilde{v}_{\phi} .
\end{aligned}
$$

We first transform this mass-squared matrix into the basis of custodial-symmetric 
states $\left(H_{5}^{+}, H_{3}^{+}, G^{+}\right)$using

$$
\mathcal{M}_{+}^{\prime 2}=R_{+} \mathcal{M}_{+}^{2} R_{+}^{T}
$$

where the orthogonal matrix $R_{+}$is defined according to

$$
\left(\begin{array}{c}
H_{5}^{+} \\
H_{3}^{+} \\
G^{+}
\end{array}\right)=R_{+}\left(\begin{array}{c}
\chi^{+} \\
\xi^{+} \\
\phi^{+}
\end{array}\right),
$$

with

$$
R_{+}=\left(\begin{array}{ccc}
\frac{1}{\sqrt{2}} & -\frac{1}{\sqrt{2}} & 0 \\
\frac{c_{H}}{\sqrt{2}} & \frac{c_{H}}{\sqrt{2}} & -s_{H} \\
\frac{s_{H}}{\sqrt{2}} & \frac{s_{H}}{\sqrt{2}} & c_{H}
\end{array}\right) .
$$

Because the custodial-symmetry-violating effects will be small, we can diagonalize the mass-squared matrix $\mathcal{M}_{+}^{\prime 2}$ using first-order perturbation theory over most of the parameter space, as detailed below. This gives some analytic insight into the structure of the custodial-symmetry-violating effects. Of course, the perturbative diagonalization only works well when the diagonal elements of the mass-squared matrix are not too degenerate; this condition is satisfied over the parameter space of the H5plane benchmark, but is violated in some regions of parameter space in our general scans. For this reason, in the next section we will use the first-order perturbative formulas below in our scans of the H5plane benchmark, but exact numerical diagonalization for our general scans. We have checked numerically that the perturbative diagonalization is a very good approximation where we use it.

To first order in the custodial violation, the masses of the singly-charged physical mass eigenstates $\tilde{H}_{5}^{+}$and $\tilde{H}_{3}^{+}$are just given by the diagonal elements of the mass- 
squared matrix,

$$
m_{\tilde{H}_{5}^{+}}^{2}=\mathcal{M}_{+, 11}^{\prime 2}, \quad m_{\tilde{H}_{3}^{+}}^{2}=\mathcal{M}_{+, 22}^{\prime 2}
$$

The compositions of the mass eigenstates are given to first order using

$$
\tilde{H}_{n}=H_{n}+\sum_{m \neq n} \frac{\mathcal{M}_{n m}^{2}}{\mathcal{M}_{n n}^{2}-\mathcal{M}_{m m}^{2}} H_{m}
$$

where $\mathcal{M}^{2}$ is the mass-squared matrix in the appropriate basis. Applying this to the singly-charged states and using the fact that $\mathcal{M}_{+, 33}^{2}=0$, we get,

$$
\begin{aligned}
\tilde{H}_{5}^{+}= & H_{5}^{+}+\frac{\mathcal{M}_{+, 12}^{\prime 2}}{\mathcal{M}_{+, 11}^{\prime 2}-\mathcal{M}_{+, 22}^{\prime 2}} H_{3}^{+}+\frac{\mathcal{M}_{+, 13}^{\prime 2}}{\mathcal{M}_{+, 11}^{\prime 2}} G^{+} \\
= & \frac{\chi^{+}-\xi^{+}}{\sqrt{2}}+\left[c_{H} \frac{\mathcal{M}_{+, 13}^{\prime 2}}{\mathcal{M}_{+, 11}^{\prime 2}}-s_{H} \frac{\mathcal{M}_{+, 12}^{\prime 2}}{\mathcal{M}_{+, 11}^{\prime 2}-\mathcal{M}_{+, 22}^{\prime 2}}\right] \phi^{+} \\
& +\left[s_{H} \frac{\mathcal{M}_{+, 13}^{\prime 2}}{\mathcal{M}_{+, 11}^{\prime 2}}+c_{H} \frac{\mathcal{M}_{+, 12}^{\prime 2}}{\mathcal{M}_{+, 11}^{\prime 2}-\mathcal{M}_{+, 22}^{\prime 2}}\right] \frac{\chi^{+}+\xi^{+}}{\sqrt{2}} \\
\tilde{H}_{3}^{+}= & H_{3}^{+}+\frac{\mathcal{M}_{+, 12}^{\prime 2}}{\mathcal{M}_{+, 22}^{\prime 2}-\mathcal{M}_{+, 11}^{\prime 2}} H_{5}^{+}+\frac{\mathcal{M}_{+, 23}^{\prime 2}}{\mathcal{M}_{+, 11}^{\prime 2}} G^{+} \\
\tilde{G}^{+}= & G^{+}+\frac{\mathcal{M}_{+, 13}^{\prime 2}}{-\mathcal{M}_{+, 11}^{\prime 2}} H_{5}^{+}+\frac{\mathcal{M}_{+, 23}^{\prime 2}}{-\mathcal{M}_{+, 22}^{\prime 2}} H_{3}^{+}
\end{aligned}
$$

We highlight the composition of $\tilde{H}_{5}^{+}$in particular because the custodial symmetry violation results in an admixture of $\phi^{+}$into this state. This allows $\tilde{H}_{5}^{+}$to couple to fermions, which does not occur in the custodial-symmetric GM model. Indeed, we can write the Feynman rule for the $\tilde{H}_{5}^{+} \bar{u} d$ vertex as

$$
\tilde{H}_{5}^{+} \bar{u} d: \quad i \frac{\sqrt{2}}{v} V_{u d} \kappa_{f}^{\tilde{H}_{5}^{+}}\left(m_{u} P_{L}-m_{d} P_{R}\right)
$$

where the coupling to fermions induced by the custodial symmetry violation is, to 
first order,

$$
\kappa_{f}^{\tilde{H}_{5}^{+}}=\frac{\mathcal{M}_{+, 13}^{\prime 2}}{\mathcal{M}_{+, 11}^{\prime 2}}-\tan \theta_{H} \frac{\mathcal{M}_{+, 12}^{\prime 2}}{\mathcal{M}_{+, 11}^{2}-\mathcal{M}_{+, 22}^{\prime 2}} .
$$

For comparison, in the custodial-symmetric GM model we can write the analogous coupling of $H_{3}^{+}$to fermion pairs as $\kappa_{f}^{H_{3}^{+}}=-\tan \theta_{H}$.

Finally, there are three CP-even neutral scalars, whose mass-squared matrix in the basis $\left(\chi^{0, r}, \xi^{0, r}, \phi^{0, r}\right)$ is given by

$$
\mathcal{M}_{r}^{2}=\left(\begin{array}{lll}
\mathcal{M}_{r, 11}^{2} & \mathcal{M}_{r, 12}^{2} & \mathcal{M}_{r, 13}^{2} \\
\mathcal{M}_{r, 12}^{2} & \mathcal{M}_{r, 22}^{2} & \mathcal{M}_{r, 23}^{2} \\
\mathcal{M}_{r, 13}^{2} & \mathcal{M}_{r, 23}^{2} & \mathcal{M}_{r, 33}^{2}
\end{array}\right)
$$

where

$$
\begin{aligned}
& \mathcal{M}_{r, 11}^{2}=-\frac{\tilde{\lambda}_{4} \tilde{v}_{\phi}^{2} \tilde{v}_{\xi}}{2 \sqrt{2} \tilde{v}_{\chi}}+4 \tilde{\lambda}_{7} \tilde{v}_{\chi}^{2}+\frac{\tilde{M}_{1}^{\prime}}{4 \tilde{v}_{\chi}} \tilde{v}_{\phi}^{2}, \\
& \mathcal{M}_{r, 22}^{2}=-\frac{\tilde{\lambda}_{4} \tilde{v}_{\phi}^{2} \tilde{v}_{\chi}}{\sqrt{2} \tilde{v}_{\xi}}+8 \tilde{\lambda}_{8} \tilde{v}_{\xi}^{2}+\frac{\tilde{M}_{1}}{4 \tilde{v}_{\xi}} \tilde{v}_{\phi}^{2}+6 \tilde{M}_{2} \frac{\tilde{v}_{\chi}^{2}}{\tilde{v}_{\xi}}, \\
& \mathcal{M}_{r, 33}^{2}= 2 \tilde{\lambda}_{1} \tilde{v}_{\phi}^{2}, \\
& \mathcal{M}_{r, 12}^{2}= \frac{\tilde{\lambda}_{4} \tilde{v}_{\phi}^{2}}{2}+2 \sqrt{2} \tilde{\lambda}_{10} \tilde{v}_{\chi} \tilde{v}_{\xi}-6 \sqrt{2} \tilde{M}_{2} \tilde{v}_{\chi}, \\
& \mathcal{M}_{r, 13}^{2}=\frac{\tilde{\lambda}_{3} \tilde{v}_{\phi} \tilde{v}_{\chi}}{\sqrt{2}}+\tilde{\lambda}_{4} \tilde{v}_{\phi} \tilde{v}_{\xi}+\sqrt{2} \tilde{\lambda}_{5} \tilde{v}_{\phi} \tilde{v}_{\chi}-\frac{\tilde{M}_{1}^{\prime} \tilde{v}_{\phi}}{\sqrt{2}}, \\
& \mathcal{M}_{r, 23}^{2}=\sqrt{2} \tilde{\lambda}_{4} \tilde{v}_{\phi} \tilde{v}_{\chi}+2 \tilde{\lambda}_{6} \tilde{v}_{\phi} \tilde{v}_{\xi}-\frac{\tilde{M}_{1} \tilde{v}_{\phi}}{2} .
\end{aligned}
$$

We first transform this mass-squared matrix into the basis of custodial-symmetric states $\left(H_{5}^{0}, H_{1}^{0 \prime}, \phi^{0, r}\right)$ using

$$
\mathcal{M}_{r}^{\prime 2}=R_{r} \mathcal{M}_{r}^{2} R_{r}^{T}
$$


where the orthogonal matrix $R_{r}$ is defined according to

$$
\left(\begin{array}{c}
H_{5}^{0} \\
H_{1}^{0 \prime} \\
\phi^{0, r}
\end{array}\right)=R_{r}\left(\begin{array}{c}
\chi^{0, r} \\
\xi^{0, r} \\
\phi^{0, r}
\end{array}\right)
$$

with

$$
R_{r}=\left(\begin{array}{ccc}
-\sqrt{\frac{1}{3}} & \sqrt{\frac{2}{3}} & 0 \\
\sqrt{\frac{2}{3}} & \sqrt{\frac{1}{3}} & 0 \\
0 & 0 & 1
\end{array}\right) .
$$

To first order in the custodial symmetry violation, the mass of $\tilde{H}_{5}^{0}$ is given by

$$
m_{\tilde{H}_{5}^{0}}^{2}=\mathcal{M}_{r, 11}^{\prime 2}
$$

It is most straightforward to find the masses of $\tilde{h}$ and $\tilde{H}$ by diagonalizing the remaining $2 \times 2$ block of $\mathcal{M}_{r}^{\prime 2}$ as follows:

$$
m_{\tilde{h}, \tilde{H}}^{2}=\frac{1}{2}\left[\mathcal{M}_{r, 33}^{\prime 2}+\mathcal{M}_{r, 22}^{\prime 2} \mp \sqrt{\left(\mathcal{M}_{r, 33}^{\prime 2}-\mathcal{M}_{r, 22}^{\prime 2}\right)^{2}+4\left(\mathcal{M}_{r, 23}^{\prime 2}\right)^{2}}\right]
$$

The mixing angle that achieves this diagonalization is given by

$$
\sin 2 \tilde{\alpha}=\frac{2 \mathcal{M}_{r, 23}^{\prime 2}}{m_{\tilde{H}}^{2}-m_{\tilde{h}}^{2}}, \quad \quad \cos 2 \tilde{\alpha}=\frac{\mathcal{M}_{r, 22}^{\prime 2}-\mathcal{M}_{r, 33}^{\prime 2}}{m_{\tilde{H}}^{2}-m_{\tilde{h}}^{2}}
$$

where the states are given in terms of $\tilde{\alpha}$ by

$$
h_{\tilde{\alpha}}=c_{\tilde{\alpha}} \phi^{0, r}-s_{\tilde{\alpha}} H_{1}^{0 \prime}, \quad H_{\tilde{\alpha}}=s_{\tilde{\alpha}} \phi^{0, r}+c_{\tilde{\alpha}} H_{1}^{0 \prime},
$$

and we have defined $c_{\tilde{\alpha}}=\cos \tilde{\alpha}, s_{\tilde{\alpha}}=\sin \tilde{\alpha}$. (Note that these are not yet the mass 
eigenstates: there is still a small mixing with $H_{5}^{0}$ to be dealt with below.) We introduce a second orthogonal rotation matrix $R_{\tilde{\alpha}}$, defined according to

$$
\left(\begin{array}{c}
H_{5}^{0} \\
H_{\tilde{\alpha}} \\
h_{\tilde{\alpha}}
\end{array}\right)=R_{\tilde{\alpha}}\left(\begin{array}{c}
H_{5}^{0} \\
H_{1}^{0 \prime} \\
\phi^{0, r}
\end{array}\right)
$$

with

$$
R_{\tilde{\alpha}}=\left(\begin{array}{ccc}
1 & 0 & 0 \\
0 & c_{\tilde{\alpha}} & s_{\tilde{\alpha}} \\
0 & -s_{\tilde{\alpha}} & c_{\tilde{\alpha}}
\end{array}\right)
$$

The mass-squared matrix in the basis $\left(H_{5}^{0}, H_{\tilde{\alpha}}, h_{\tilde{\alpha}}\right)$ is then given by

$$
\mathcal{M}_{r}^{\prime \prime 2}=R_{\tilde{\alpha}} \mathcal{M}_{r}^{\prime 2} R_{\tilde{\alpha}}^{T}=\left(\begin{array}{ccc}
\mathcal{M}_{r, 11}^{\prime \prime 2} & \mathcal{M}_{r, 12}^{\prime 2} & \mathcal{M}_{r, 13}^{\prime \prime 2} \\
\mathcal{M}_{r, 12}^{\prime \prime 2} & \mathcal{M}_{r, 22}^{\prime 2} & 0 \\
\mathcal{M}_{r, 13}^{\prime \prime 2} & 0 & \mathcal{M}_{r, 33}^{\prime \prime 2}
\end{array}\right)
$$

Note that $\mathcal{M}_{r, 11}^{\prime 2}=\mathcal{M}_{r, 11}^{\prime 2}$. The masses of $\tilde{h}$ and $\tilde{H}$ can then be written (to first order in the custodial symmetry violation) in terms of the diagonal elements of this matrix as

$$
m_{\tilde{h}}^{2}=\mathcal{M}_{r, 33}^{\prime \prime 2}, \quad m_{\tilde{H}}^{2}=\mathcal{M}_{r, 22}^{\prime \prime 2}
$$

We now use Eq. (4.32) to write the compositions of the CP-even neutral mass eigenstates to first order in the custodial violation as

$$
\begin{aligned}
\tilde{H}_{5}^{0} & =H_{5}^{0}+\frac{\mathcal{M}_{r, 12}^{\prime \prime 2}}{\mathcal{M}_{r, 11}^{\prime \prime 2}-\mathcal{M}_{r, 22}^{\prime \prime 2}} H_{\tilde{\alpha}}+\frac{\mathcal{M}_{r, 13}^{\prime \prime 2}}{\mathcal{M}_{r, 11}^{\prime \prime 2}-\mathcal{M}_{r, 33}^{\prime 2}} h_{\tilde{\alpha}} \\
& =\left(\sqrt{\frac{2}{3}} \xi^{0, r}-\sqrt{\frac{1}{3}} \chi^{0, r}\right)+\left[s_{\tilde{\alpha}} \frac{\mathcal{M}_{r, 12}^{\prime 2}}{\mathcal{M}_{r, 11}^{\prime \prime 2}-\mathcal{M}_{r, 22}^{\prime \prime 2}}+c_{\tilde{\alpha}} \frac{\mathcal{M}_{r, 13}^{\prime 2}}{\mathcal{M}_{r, 11}^{\prime \prime 2}-\mathcal{M}_{r, 33}^{\prime \prime 2}}\right] \phi^{0, r}
\end{aligned}
$$




$$
\begin{aligned}
& +\left[c_{\tilde{\alpha}} \frac{\mathcal{M}_{r, 12}^{\prime \prime 2}}{\mathcal{M}_{r, 11}^{\prime \prime 2}-\mathcal{M}_{r, 22}^{\prime \prime 2}}-s_{\tilde{\alpha}} \frac{\mathcal{M}_{r, 13}^{\prime \prime 2}}{\mathcal{M}_{r, 11}^{\prime 2}-\mathcal{M}_{r, 33}^{\prime \prime 2}}\right]\left(\sqrt{\frac{1}{3}} \xi^{0, r}+\sqrt{\frac{2}{3}} \chi^{0, r}\right), \\
\tilde{H}= & H_{\tilde{\alpha}}+\frac{\mathcal{M}_{r, 12}^{\prime \prime 2}}{\mathcal{M}_{r, 22}^{\prime \prime 2}-\mathcal{M}_{r, 11}^{\prime \prime 2}} H_{5}^{0}, \\
\tilde{h}= & h_{\tilde{\alpha}}+\frac{\mathcal{M}_{r, 13}^{\prime \prime 2}}{\mathcal{M}_{r, 33}^{\prime \prime 2}-\mathcal{M}_{r, 11}^{\prime \prime 2}} H_{5}^{0} .
\end{aligned}
$$

We highlight the composition of $\tilde{H}_{5}^{0}$ in particular because the custodial symmetry violation results in an admixture of $\phi^{0, r}$ into this state. This allows $\tilde{H}_{5}^{0}$ to couple to fermions, which does not occur in the custodial-symmetric GM model. The coupling of $\tilde{H}_{5}^{0}$ to $\bar{f} f$, normalized to the corresponding coupling of the SM Higgs boson, is then given to first order in the custodial symmetry violation by

$$
\kappa_{f}^{\tilde{H}_{5}^{0}}=\frac{1}{c_{H}}\left[s_{\tilde{\alpha}} \frac{\mathcal{M}_{r, 12}^{\prime \prime 2}}{\mathcal{M}_{r, 11}^{\prime \prime 2}-\mathcal{M}_{r, 22}^{\prime \prime 2}}+c_{\tilde{\alpha}} \frac{\mathcal{M}_{r, 13}^{\prime \prime 2}}{\mathcal{M}_{r, 11}^{\prime 2}-\mathcal{M}_{r, 33}^{\prime \prime 2}}\right]
$$

Finally, the mixing of a small amount of custodial-fiveplet $H_{5}^{0}$ into the physical Higgs boson $\tilde{h}$, together with $\tilde{v}_{\chi} \neq \tilde{v}_{\xi}$, leads to a violation of custodial symmetry in the couplings of $\tilde{h}$ to $W W$ and $Z Z$. This is parameterized in terms of the physical observable

$$
\lambda_{W Z}^{\tilde{h}} \equiv \frac{\kappa_{W}^{\tilde{h}}}{\kappa_{Z}^{\tilde{h}}},
$$

where $\kappa_{W}^{\tilde{h}}$ and $\kappa_{Z}^{\tilde{h}}$ are the couplings of $\tilde{h}$ to $W W$ and $Z Z$, respectively, normalized to the corresponding couplings of the SM Higgs boson. We can write this in terms of the vevs and the mixing with $H_{5}^{0}$ as follows:

$$
\lambda_{W Z}^{\tilde{h}}=\frac{\tilde{\kappa}_{W}^{h_{\tilde{\alpha}}}+\epsilon \tilde{\kappa}_{W}^{H_{5}^{0}}}{\tilde{\kappa}_{Z}^{h_{\tilde{z}}}+\epsilon \tilde{\kappa}_{Z}^{H_{5}^{0}}},
$$

where the couplings of $h_{\tilde{\alpha}}$ to $W$ and $Z$ boson pairs, including the effects of $\tilde{v}_{\chi} \neq \tilde{v}_{\xi}$, 
are given by

$$
\tilde{\kappa}_{W}^{h_{\tilde{\alpha}}}=c_{\tilde{\alpha}} \frac{\tilde{v}_{\phi}}{v}-s_{\tilde{\alpha}} \frac{4}{\sqrt{3}} \frac{\tilde{v}_{\chi}+\tilde{v}_{\xi}}{v}, \quad \tilde{\kappa}_{Z}^{h_{\tilde{\alpha}}}=c_{\tilde{\alpha}} \frac{\tilde{v}_{\phi}}{v}-s_{\tilde{\alpha}} \frac{8}{\sqrt{3}} \frac{\tilde{v}_{\chi}}{v},
$$

the couplings of $H_{5}^{0}$ to $W$ and $Z$ boson pairs are given by

$$
\tilde{\kappa}_{W}^{H_{5}^{0}}=\sqrt{\frac{2}{3}} \frac{4 \tilde{v}_{\xi}-2 \tilde{v}_{\chi}}{v}=\frac{1}{\sqrt{3}} s_{H}+\mathcal{O}(\Delta \rho), \quad \tilde{\kappa}_{Z}^{H_{5}^{0}}=-\sqrt{\frac{2}{3}} \frac{4 \tilde{v}_{\chi}}{v} \simeq-\frac{2}{\sqrt{3}} s_{H}+\mathcal{O}(\Delta \rho),
$$

and the mixing of $H_{5}^{0}$ into $\tilde{h}$ from Eq. (4.53) is

$$
\epsilon=\frac{\mathcal{M}_{r, 13}^{\prime \prime 2}}{\mathcal{M}_{r, 33}^{\prime \prime 2}-\mathcal{M}_{r, 11}^{\prime \prime 2}}=\mathcal{O}(\Delta \rho)
$$

\subsection{Numerical results}

\subsubsection{Calculational procedure}

In this thesis we imagine that the custodially-symmetric GM model emerges at some scale $\Lambda$ as an effective theory of some unspecified ultraviolet (UV) completion. For example, the scalars in the GM model could be composites and the custodial symmetry an accidental global symmetry resulting from the particle content of the UV theory. The running of the scalar potential parameters down to the weak scale induces custodial symmetry violation. We can then use the experimental constraint on the $\rho$ parameter at the weak scale to set an upper bound on the scale $\Lambda$. Subject to this constraint, we can also predict the size of other custodial symmetry violating effects such as mass splittings among the members of the custodial fiveplet and triplet scalars, mixing between scalars in different custodial-symmetry representations (which, for example, can induce fermionic decays of the otherwise fermiophobic $H_{5}$ 
states), and the value of the ratio $\lambda_{W Z} \equiv \kappa_{W} / \kappa_{Z}$ of the $125 \mathrm{GeV}$ Higgs boson (predicted as $\lambda_{W Z}=1$ in custodial-symmetric theories). Since we want to compare the custodial symmetric and custodial violating theories, we begin our procedure with the custodial symmetric theory at the weak scale. To relate this parameter point to the custodial symmetric UV scale theory we use the custodial symmetric RGEs, with $g^{\prime}$ set to zero, to run to the UV scale. When we run back down, we can compare the corresponding custodial violating parameter point to the original custodial symmetric point.

For concreteness, we start our analysis within the context of the so-called H5plane benchmark, the two-dimensional slice through the custodial-symmetric GM model parameter space defined in Table 3.1 in Sec. 3.3.2 at the weak scale. Recall that the H5plane benchmark takes $m_{5}$ and $s_{H}$ as its two free parameters: this will allow us to plot our results as contours in the $m_{5}-s_{H}$ plane. The benchmark is defined for $m_{5}$ values of $200 \mathrm{GeV}$ and higher. To test the generality of our results in the H5plane benchmark we then perform a general parameter scan and compare the results to the benchmark region. The parameter points are generated and checked for theoretical consistency using the public code GMCALC [76]. The input parameters used in the general scan are given in Table 4.1. We finally perform a second dedicated parameter scan focusing on $m_{5}$ masses below $200 \mathrm{GeV}$, to cover the region in which the H5plane benchmark is not defined. A dedicated scan is needed in this region because only a small fraction of scanned points satisfy the theoretical constraints. The input parameters used in this dedicated low $m_{5}$ scan are given in Table 4.2. Results of this dedicated scan are collected in Sec. 4.3.6. The results given in this section were calculated using my own custom-written $\mathrm{C}++$ code which was interfaced with the GMCALC routines used to generate the tree-level parameter points 


\begin{tabular}{ll}
\hline \hline Fixed Parameters & Variable Parameters \\
\hline$G_{F}=1.1663787 \times 10^{-5} \mathrm{GeV}^{-2}$ & $\mu_{3}^{2} \in\left[-(200 \mathrm{GeV})^{2},(4200 \mathrm{GeV})^{2}\right]$ \\
$m_{h}=125 \mathrm{GeV}$ & $\lambda_{2}, \lambda_{3}, \lambda_{4}, \lambda_{5}$ \\
& $M_{1}, M_{2}$ \\
\hline \hline
\end{tabular}

Table 4.1: Input parameters for the general scan in the custodial-symmetric GM model.

\begin{tabular}{ll}
\hline \hline Fixed Parameters & Variable Parameters \\
\hline$G_{F}=1.1663787 \times 10^{-5} \mathrm{GeV}^{-2}$ & $m_{5}<200 \mathrm{GeV}$ \\
$m_{h}=125 \mathrm{GeV}$ & $s_{H} \in(0,1)$ \\
& $\lambda_{2}, \lambda_{3}, \lambda_{4}, \lambda_{5}$ \\
& $M_{2} \in[-1200 \mathrm{GeV}, 1200 \mathrm{GeV}]$ \\
\hline \hline
\end{tabular}

Table 4.2: Input parameters for the dedicated low- $m_{5}$ scan in the custodialsymmetric GM model.

and apply the appropriate theoretical constraints. My numerical implementation was cross-checked for particular test points by the independent numerical implementation of my collaborator Heather Logan.

We perform the calculations as follows. We start by specifying an input point in the custodial-symmetric GM model at the weak scale. Because it is not possible to separate the scale of the GM model states from the SM weak scale so long as the triplets contribute to electroweak symmetry breaking, for the purposes of renormalization group running we will define the "weak scale" to be $m_{5}$ as defined in the custodial-symmetric low-scale input parameter set. We define the electroweak gauge couplings at the weak scale in terms of the inputs $G_{F}, M_{W}$, and $M_{Z}$, and we take $\alpha_{s}\left(M_{Z}\right)=0.118$ to define the strong coupling at the weak scale (we ignore the running of the strong coupling between $M_{Z}$ and $m_{5}$; this is a small effect because the strong coupling only enters in the running of the top Yukawa coupling). We extract the value of the top Yukawa coupling using the relation $y_{t}=\sqrt{2} m_{t} / v_{\phi}$ evaluated in 
terms of the custodial-symmetric input parameters at the weak scale. For simplicity, we set $y_{b}=y_{\tau}=0$; their effects would be very small. This parameterization of the weak scale means that the matching scale varies throughout the parameter space. As is pointed out in Sec. 2.5, the ambiguity introduced by this choice is higher order than our leading log approximation.

From the tree-level custodial symmetric model, we then run the parameters of the custodial-symmetric scalar potential up to a scale $\Lambda$ using the RGEs in Eqs. (A.1A.16) but with $g_{1}$ set to zero. (Where $g_{1}=\sqrt{\frac{5}{3}} g^{\prime}$ is the hypercharge coupling using the GUT normalization.) We also run the gauge couplings (including the actual value of $g_{1}$ ) and the top Yukawa coupling from $m_{5}$ to $\Lambda$ using Eqs. (A.18-A.21). For the running we use fourth-order Runge-Kutta with a small step size of 0.001 for the variable $t \equiv \log (\mu)$ (all logs being natural $\operatorname{logs}$ ). ${ }^{1}$ The result of this is a custodial-symmetric scalar potential at the scale $\Lambda$. At this stage we can check whether any of the quartic scalar couplings has grown large enough to violate perturbative unitarity (indicating that we have almost run into a Landau pole). This allows us to determine the maximum scale allowed by perturbativity. We also check whether the potential has become unbounded from below; this turns out not to happen for any of our scan points in the H5plane benchmark or in the general parameter scans. Because the potential is still custodial-symmetric, we can use the requirements for perturbative unitarity and boundedness-from-below as derived for the custodial-symmetric theory [74] as given at the end of Sec. 3.1.

From the custodial-symmetric scalar potential at scale $\Lambda$, we then run back down to the scale $m_{5}$ using the full RGEs in Eqs. (A.1-A.21) with $g_{1} \neq 0$. The

\footnotetext{
${ }^{1}$ We chose this particular step size by starting with a step size of 0.1 and decreased the step size by an order of magnitude until it did not significantly change our numerical solutions for several test points. As our numerical procedure could be easily compartmentalized we did not have to worry about trading Runge-Kutta precision for run time. Running in the background on a laptop the run time for each piece of our code was in the neighbourhood of $24-48$ hours.
} 
nonzero hypercharge coupling induces custodial symmetry violation in the scalar potential, causing violation of the custodial-symmetry relations of Eq. (4.15) among the parameters of the most general gauge invariant scalar potential. Having determined the custodial violating parameters we can now solve the minimization conditions in Eqs. (4.17), (4.18), and (4.19) for the custodial-violating vevs $\tilde{v}_{\phi}, \tilde{v}_{\chi}$, and $\tilde{v}_{\xi}$. Unlike the custodial symmetric case, we cannot easily find an analytic expression for the vevs. Therefore, we first solve Eq. (4.17) for $\tilde{v}_{\phi}^{2}$ in terms of the other vevs and plug this in to Eqs. (4.18) and (4.19), which we then solve numerically using a twodimensional Newton's method. For the initial guess we take $\tilde{v}_{\chi}=\tilde{v}_{\xi}=v_{\chi}$, where $v_{\chi}$ is the custodial-symmetric triplet vev in our original weak-scale input point.

However, this procedure suffers from a complication. The definition of the original weak scale input point uses the measured $m_{h}$ and $G_{F}$ as input parameters. These are used to fix $\lambda_{1}$ and $\mu_{2}^{2}$ in the weak-scale custodial-symmetric theory. After running the parameters up to the scale $\Lambda$ using the custodial-symmetric RGEs (with $g^{\prime}$ set to zero) and then running them back down to the weak scale with the full custodial violating RGEs, the new weak-scale calculations of $m_{\tilde{h}}$ and $G_{F}^{-1}=\sqrt{2}\left(\tilde{v}_{\phi}^{2}+4 \tilde{v}_{\chi}^{2}+4 \tilde{v}_{\xi}^{2}\right)$ yield numbers that do not match the original input values. To address this, we need to adjust the custodially-symmetric weak-scale input values for $\lambda_{1}$ and $\mu_{2}^{2}$ (while keeping all the other weak-scale inputs fixed) until we obtain the correct experimental values of $m_{\tilde{h}}$ and $G_{F}$ after implementing the custodial symmetry violation. We do this by defining two functions, $f_{1}=m_{\tilde{h}}^{\text {calc }}\left(\lambda_{1}, \mu_{2}^{2}\right)-m_{h}^{\text {expt }}$ and $f_{2}=G_{F}^{c a l c}\left(\lambda_{1}, \mu_{2}^{2}\right)-G_{F}^{\text {expt }}$, where $\lambda_{1}$ and $\mu_{2}^{2}$ are the inputs at the weak scale, $m_{\tilde{h}}^{\text {calc }}$ and $G_{F}^{\text {calc }}$ are calculated using the procedure described above, and $m_{h}^{\text {expt }}$ and $G_{F}^{\text {expt }}$ are the desired (experimental) values. The solution is the point at which $f_{1}=f_{2}=0$, which we find iteratively using a two-dimensional Newton's method. This involves running the full RGE machinery 
up and down multiple times and is the slowest part of our numerical work. The same is generically true for $m_{t}$ (which we use to fix the top quark Yukawa coupling $y_{t}$ at the weak scale). In the H5plane benchmark, the change to $m_{t}$ after running up and back down again is within the current experimental error so we ignore this effect. In the general parameter scans, however, the change in $m_{t}$ can be larger, so in these scans we extend the iterative procedure to include $y_{t}$.

Having solved for the appropriate input values of $\lambda_{1}$ and $\mu_{2}^{2}$, we now have a self-consistent set of scalar potential input parameters at the weak scale $\left(\mu=m_{5}\right)$, corresponding to a custodial-symmetric theory at the high scale $(\mu=\Lambda)$, which we then run back down to obtain the custodial-violating theory at the weak scale (again $\left.m_{5}\right)$ with the correct predictions for $m_{h}$ and $G_{F}$. We then calculate our desired observables including the $\rho$ parameter, the mass splittings among the states of the would-be custodial multiplets, and the effects of the mixing among the would-be custodial eigenstates.

In the rest of this section we present our results as contour plots in the H5plane benchmark in the $m_{5}-s_{H}$ plane, and as scatter plots for the general scans. To calculate our contour plots we sample the H5plane benchmark according to a fixed grid. When we compare our benchmark results to our general scans results, we use our results from this grid; we do not also randomly scan the H5plane benchmark. We emphasize that $m_{5}$ and $s_{H}$ here are defined as part of the weak-scale custodial-symmetric input parameter point, and do not directly correspond to the physical masses, couplings, or vevs of the corresponding parameter point in the weak-scale custodial-violating theory. However, as we will show in what follows, the deviations of these physical observables from the custodial-symmetric input parameters are small enough that the differences are unlikely to be observable at the LHC. 


\subsubsection{Constraints on the cutoff scale from perturbativity and the $\rho$ parameter}

We begin by determining the maximum scale allowed for the custodial-symmetric ultraviolet completion by running the custodial-symmetric model up until we hit a Landau pole. This is shown in the top panel of Fig. 4.1 in the H5plane benchmark. The shaded region at large $s_{H}$ in these plots is excluded by theoretical constraints on the custodial-symmetric model. We define the Landau pole as the scale at which any of the custodial-symmetric quartic couplings $\lambda_{i}$ becomes larger than $10^{3}$; the true divergence happens extremely close to this scale. Landau poles occur when the perturbative expansion becomes unstable, so it is possible that working to higher order in perturbation theory could give sizable corrections to our 1-loop results for the scale of the Landau pole. Since higher order effects only become significant once the couplings become nonperturbative, they are unlikely to have as large an impact on the scale where perturbative unitarity is violated. In the bottom panel of Fig. 4.1 we also show the scale at which the quartic couplings in the custodial-symmetric theory violate any of the conditions for perturbative unitarity of two-to-two scattering amplitudes given in Eq. (3.20). We can see that the scale at which perturbative unitarity is violated is roughly an order of magnitude below the scale of the Landau pole. Within the H5plane benchmark, if the theory is to remain perturbative the ultraviolet completion has to appear at $290 \mathrm{TeV}$ or below, and the maximum scale of the Landau pole in this benchmark is around $2600 \mathrm{TeV}$. For $m_{5} \gtrsim 400 \mathrm{GeV}$, the upper bound on $s_{H}$ from theory constraints in the H5plane benchmark is due to the perturbative unitarity constraint; therefore along this boundary the scale of perturbative unitarity violation is essentially the same as $m_{5}$, and the Landau pole occurs around $10 \mathrm{TeV}$. 
We also note that in the H5plane benchmark, the value of $\lambda_{2}$ at the weak scale grows linearly with $m_{5}$ (see Table 3.1). This is responsible for the decrease in the scale of perturbative unitarity violation and the subsequent Landau pole with increasing $m_{5}$ at small $s_{H}$ values, and is a feature of the H5plane benchmark.

In all the scans that follow, we take the scale of perturbative unitarity violation to be an upper bound on the scale of the custodial-symmetric theory, and we do not run above this scale.

The maximum allowed scale of the custodial-symmetric ultraviolet completion can also be constrained by the stringent experimental limits on the $\rho$ parameter, as defined in Eq. (4.10). For this calculation (and those that follow), we bring to bear the full computational machinery described in the previous section, including adjusting the input values of $\lambda_{1}$ and $\mu_{2}^{2}$ to obtain the correct measured values of $G_{F}$ and $m_{h}$ in the custodial-violating theory at the weak scale. We take the value of $\rho$ from the 2016 Particle Data Group electroweak fit $[98]^{2}$,

$$
\rho=1.00037 \pm 0.00023
$$

and require that the value of $\rho$ in the weak-scale custodial-violating theory be within $2 \sigma$ of this value; i.e., between $\rho_{\text {lower }}=0.99991$ and $\rho_{\text {upper }}=1.00083$. Because the deviation in the $\rho$ parameter in the custodial-violating weak-scale theory grows as the scale of the custodial-symmetric ultraviolet completion increases, this constraint puts a stronger upper bound on the scale of the ultraviolet completion in part of the H5plane benchmark parameter space, as shown in the top panel of Fig. 4.2, where we also plot the upper bound from requiring perturbative unitarity. The $\rho$ parameter

\footnotetext{
${ }^{2}$ The current value of $\rho=1.00038 \pm 0.00020$ from the 2020 Particle Data Group electroweak fit [4] is close enough to the 2016 value to scarcely impact our results.
} 

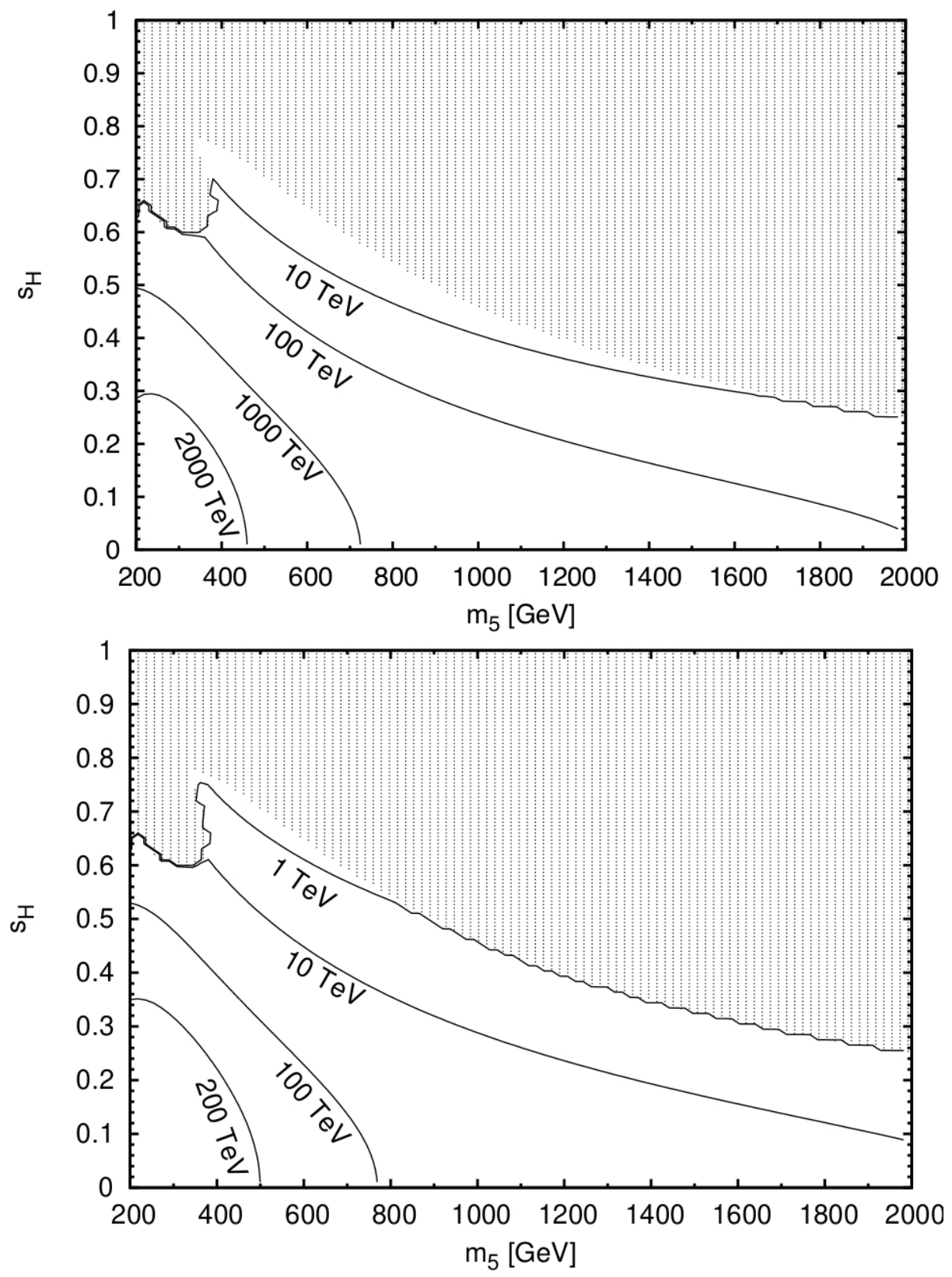

Figure 4.1: Constraints on the custodial-symmetric cutoff scale due to perturbativity of the model in the H5plane benchmark. Top: the scale of the Landau pole, defined as the scale at which any of the $\lambda_{i}$ in the custodial-symmetric theory becomes larger than $10^{3}$. This scale varies between $2.5 \mathrm{TeV}$ and $2594.2 \mathrm{TeV}$ over the benchmark considered. Bottom: the highest scale at which the perturbative unitarity constraints of Eq. (3.20) in the custodial-symmetric theory remain satisfied. This scale varies between $346.8 \mathrm{GeV}$ and $291.1 \mathrm{TeV}$ over the benchmark considered. 
constraint is stronger than that from perturbative unitarity for moderate $s_{H}$ values and $m_{5}$ below about $850 \mathrm{GeV}$.

In the bottom panel of Fig. 4.2 we plot contours of $\rho$ at the weak scale in the custodial-violating theory after running down from the maximum scale allowed by the stronger of the perturbative unitarity and $\rho$ parameter constraints. $\rho>1$ in almost all of the H5plane benchmark, except for a tiny sliver of parameter space at low $m_{5}<250 \mathrm{GeV}$ and $s_{H}$ below 0.4 . While the contours tend to be smooth throughout the plane, there are several regions, especially at low $m_{5}$ and high $s_{H}$ which are quite rough. As this roughness also appears in most of our other contours in the H5plane benchmark plane a brief discussion on the probable origin of these distortions is in order.

To make these contours, we divided the H5plane benchmark into a 100 by 100 grid with $m_{5}$ steps size of $18 \mathrm{GeV}$ and $s_{H}$ step size of 0.01 and generated points along this grid to use as our initial weak scale inputs. Some of the roughness and the appearance of islands of contour levels is due to contouring effects but these artifacts appear around either a single point or a small number of points with atypical values of $\rho$ compared to their local neighbourhood of points. These points are scattered around the low $m_{5}$ and high $s_{H}$ region for $\rho=1.0008$ which generates the large number of islands seen in the figure. Away from this region, there are small regions of a couple of grid points where the calculated value of $\rho$ drops compared to their immediate region. These are responsible for the small bumps and the occasional island which appear along the otherwise smooth contours at larger values of $m_{5}$ such as those that appear in the 1.0002 contour between $m_{5} \sim 1100-1300 \mathrm{GeV}$. These atypical points are probably a result of our calculation procedure. Although all our initial input points are defined in the H5plane benchmark, as $m_{5}$ and $s_{H}$ do not 
correspond to physical parameters, in some sense the benchmark plane cannot be well-defined in the custodial violating weak scale theory. Furthermore, our process of running the custodial symmetric weak scale parameters up to the cutoff scale, $\Lambda$, and then matching to the weak scale custodial violating theory can be thought of as our parameter points flowing away from the H5plane in some direction through parameter space and then returning via another similar path. When we fix our inputs $m_{h}$ and $G_{F}$ by solving our non-linear system of equations, we are choosing which direction in the (now unphysical) custodial symmetric parameter space we drift away from the H5plane benchmark. This drifting continues until we find our actual weak scale input point which fixes the path followed by the RGE flow to our UV input point. As this is a non-linear solution, it is possible that very similar input points in the H5plane can correspond to UV input points which are further away from each other than their apparent position in the H5plane might suggest. The occasional roughness and islands which appear in our contours are thus to be expected.

In Fig. 4.3 we show scatter plots comparing the perturbative unitarity constraints in the H5plane benchmark to the results of the general scan. In the top panel we plot the maximum cutoff scale $\Lambda$ versus $m_{5}$ while in the bottom panel we plot it versus $s_{H}$. For high values of $m_{5}$ we find that the H5plane benchmark gives cutoff scales lower than are typical in the general scan. This is expected because the quartic coupling $\lambda_{2}$ grows with $m_{5}$ in the H5plane benchmark, putting those points closer to the limit from perturbative unitarity. For lower $m_{5}$ values, the H5plane benchmark tends to give cutoff scales which are larger than typical in the general scan. This is a statistical effect caused by the fact that much of the parameter space in the general scan tends to have one or more quartic coupling already moderately large, while in the H5plane benchmark specific (smaller) parameter values have been chosen by hand. 

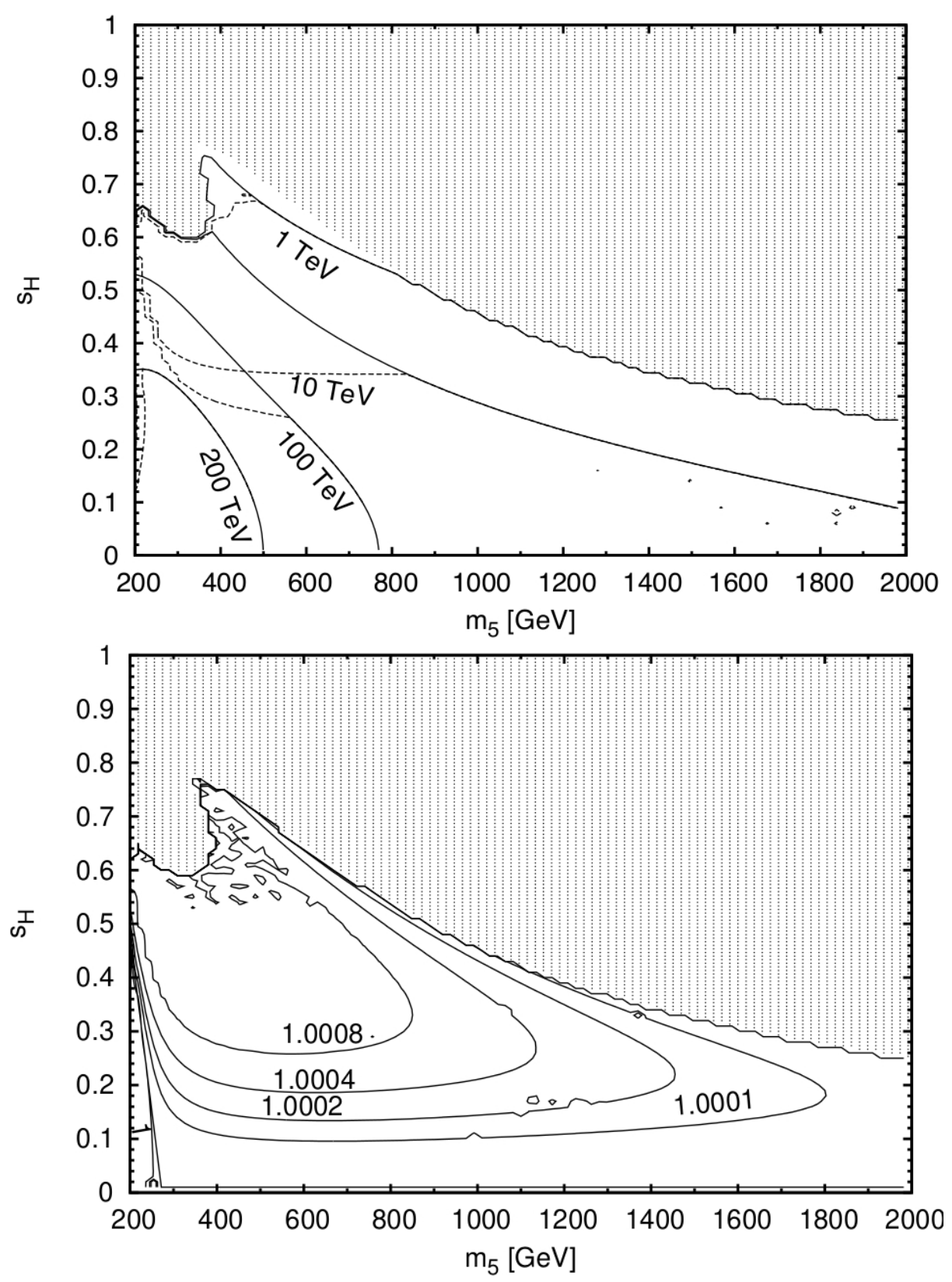

Figure 4.2: Values of and constraints due to the $\rho$ parameter in the H5plane benchmark. Top: the highest scale at which the perturbative unitarity constraints of Eq. (3.20) in the custodial-symmetric theory remain satisfied as in the right panel of Fig. 4.1 (solid lines), showing also the highest allowed custodial-symmetric scale after requiring that the $\rho$ parameter remain within $\pm 2 \sigma$ of its experimental value [Eq. (4.60)] in the custodial-violating weak-scale theory (dashed lines). The range of scales allowed after imposing the constraint on $\rho$ remains the same as in Fig. 4.1. Bottom: the value of $\rho$ in the weak-scale custodial-violating theory when the custodialsymmetric scale is taken as large as possible subject to perturbative unitarity at the high scale and the experimental limits on $\rho$. The values of $\rho$ range between the $\pm 2 \sigma$ limits of 0.99991 and 1.00083 . 
Similarly, points in the H5plane benchmark yield higher maximum cutoff scales than typical points in the general scan for all values of $s_{H}$. The points in the general scan with very high maximum cutoff scales cluster at low $s_{H}$ below 0.2 .

In Fig. 4.4 we again show scatter plots comparing the maximum cutoff scale in the H5plane benchmark to the results of the general scan, now imposing the requirement that the $\rho$ parameter at the weak scale is within $2 \sigma$ of its experimental value in addition to the perturbative unitarity requirement. The $\rho$ parameter constraint lowers the maximum allowed cutoff scale in both the H5plane benchmark and the general scan, and brings the two distributions closer to each other. At lower $m_{5}$ values the H5plane benchmark still permits somewhat atypically large cutoffs but it gives mostly typical cutoff values for higher values of $s_{H}$. The general scan still admits higher cutoff scales than the H5plane benchmark (particularly at large $m_{5}$ ), but the highest cutoff scale in the general scan is now less than an order of magnitude higher than that in the H5plane benchmark.

In Fig. 4.5 we plot the value of the weak-scale $\rho$ parameter when the cutoff scale is at its maximum value allowed by perturbative unitarity and the experimental constraints on $\rho$ in the H5plane benchmark and the general scan. We see that, as in the H5plane benchmark, the general scan yields $\rho \geq 1$ in the overwhelming majority of parameter space (as mildly favoured by experiment). Indeed, the region at very low $m_{5}$ in the H5plane benchmark in which $\rho<1$ is quite atypical in the general scan. The general scan also tends to give slightly larger values of $\rho$ at higher $m_{5}$, as one would expect given the higher maximum cutoff scales (and hence more custodialsymmetry-violation-inducing running) in this mass range in the general scan. 

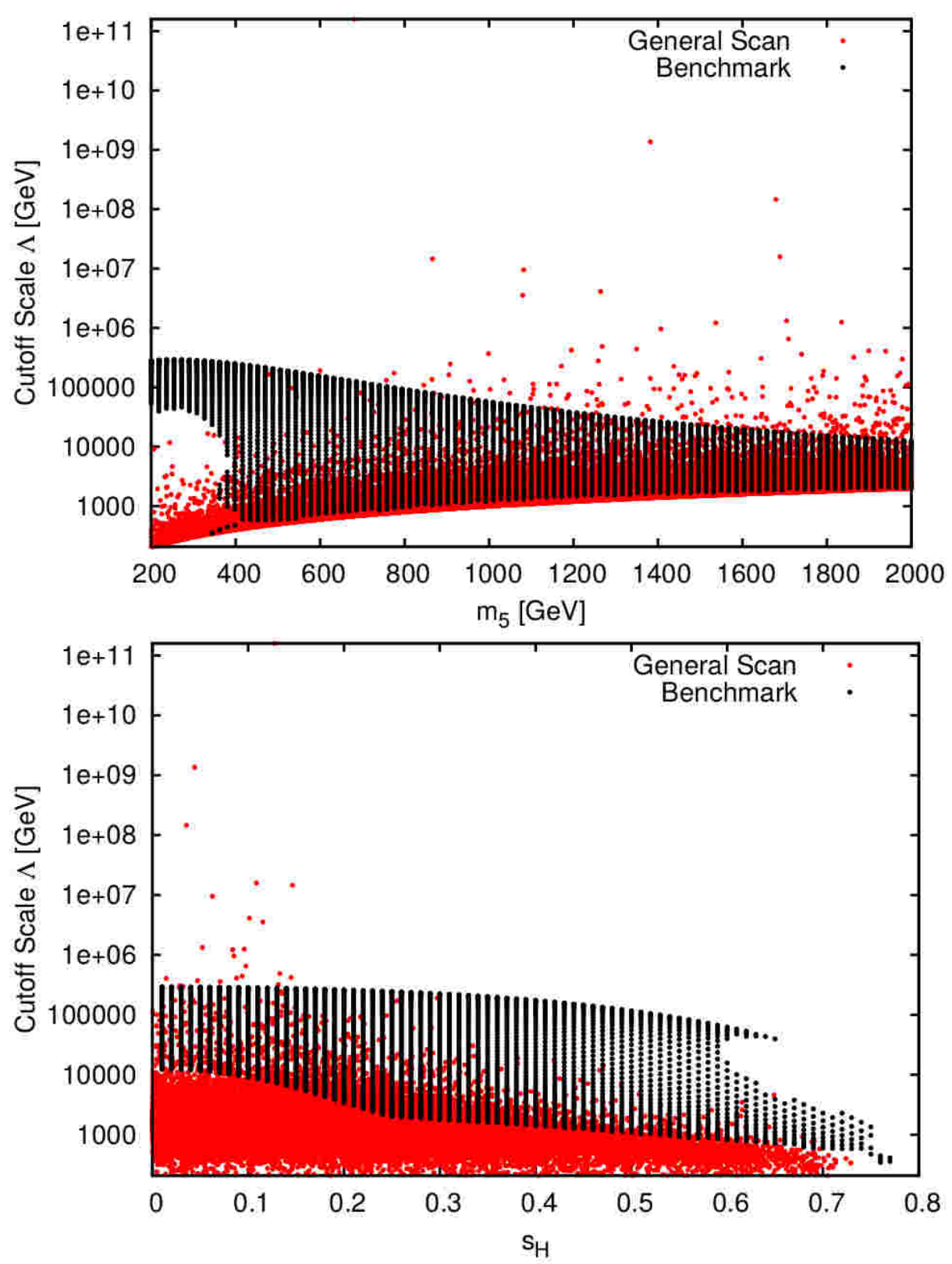

Figure 4.3: The highest allowed custodial-symmetric cutoff scale due to perturbative unitarity of the quartic couplings in a general scan (red) and in the H5plane benchmark (black), as a function of $m_{5}$ (top) and $s_{H}$ (bottom). The highest allowed cutoff scale in the general scan ranges between $207 \mathrm{GeV}$ and $1.6 \times 10^{11} \mathrm{GeV}$, though almost all points lie below $\sim 2 \times 10^{9} \mathrm{GeV}$. (The point with the highest cutoff scale is at the upper edge of the plots at $m_{5}=681 \mathrm{GeV}$ and $s_{H} \simeq 0.12$ ). 

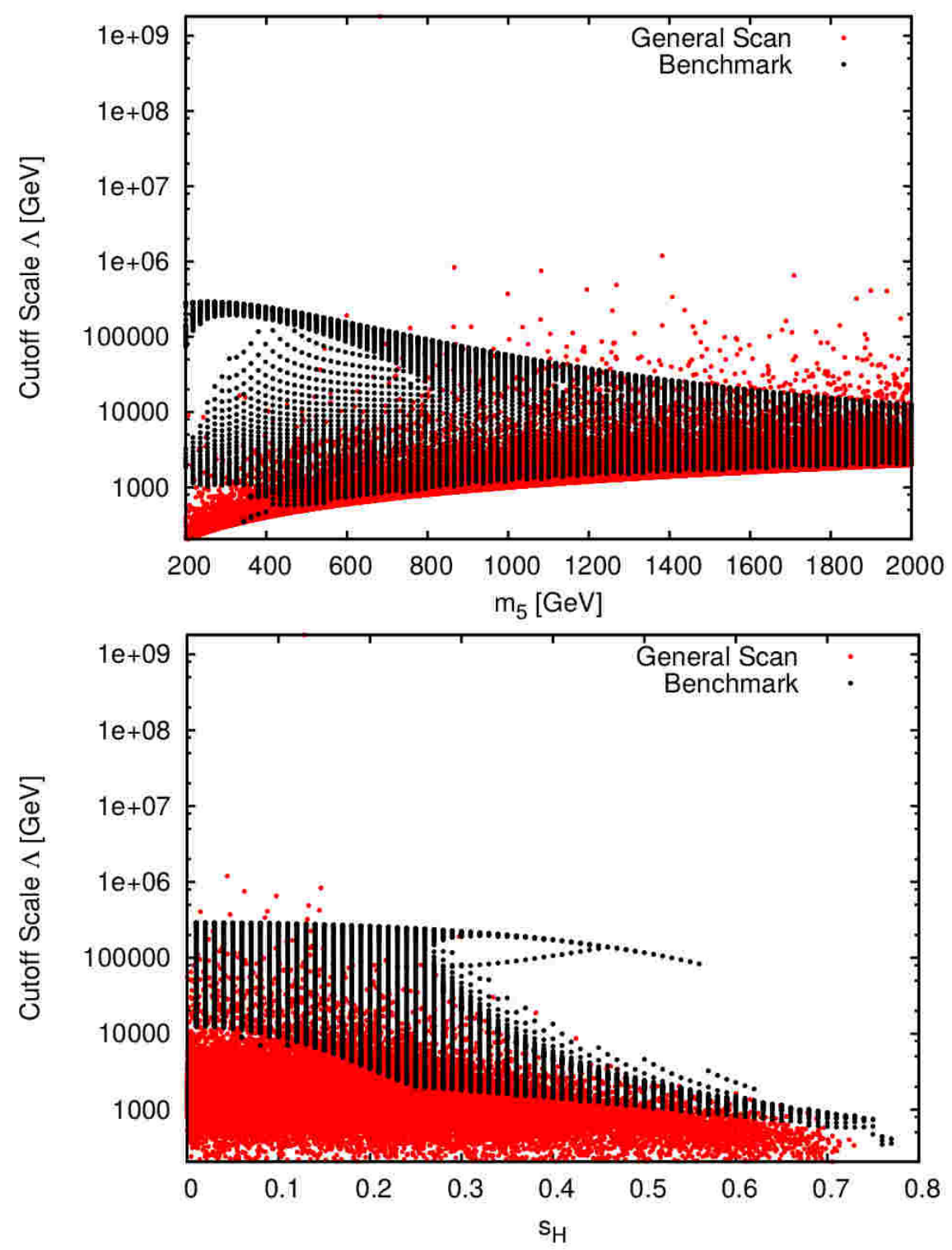

Figure 4.4: The highest allowed custodial-symmetric cutoff scale imposing perturbative unitarity and the requirement that the weak-scale $\rho$ parameter lie within $\pm 2 \sigma$ of its measured value. Red points are for a general scan and black are for the H5plane benchmark, as a function of $m_{5}$ (top) and $s_{H}$ (bottom). The highest allowed cutoff scale in the general scan ranges between $205 \mathrm{GeV}$ and $1.8 \times 10^{9} \mathrm{GeV}$, though almost all the points lie below $\sim 2 \times 10^{6} \mathrm{GeV}$. (The point with the highest cutoff scale is at the upper edge of the plots at $m_{5}=681 \mathrm{GeV}$ and $s_{H} \simeq 0.12$ ). 

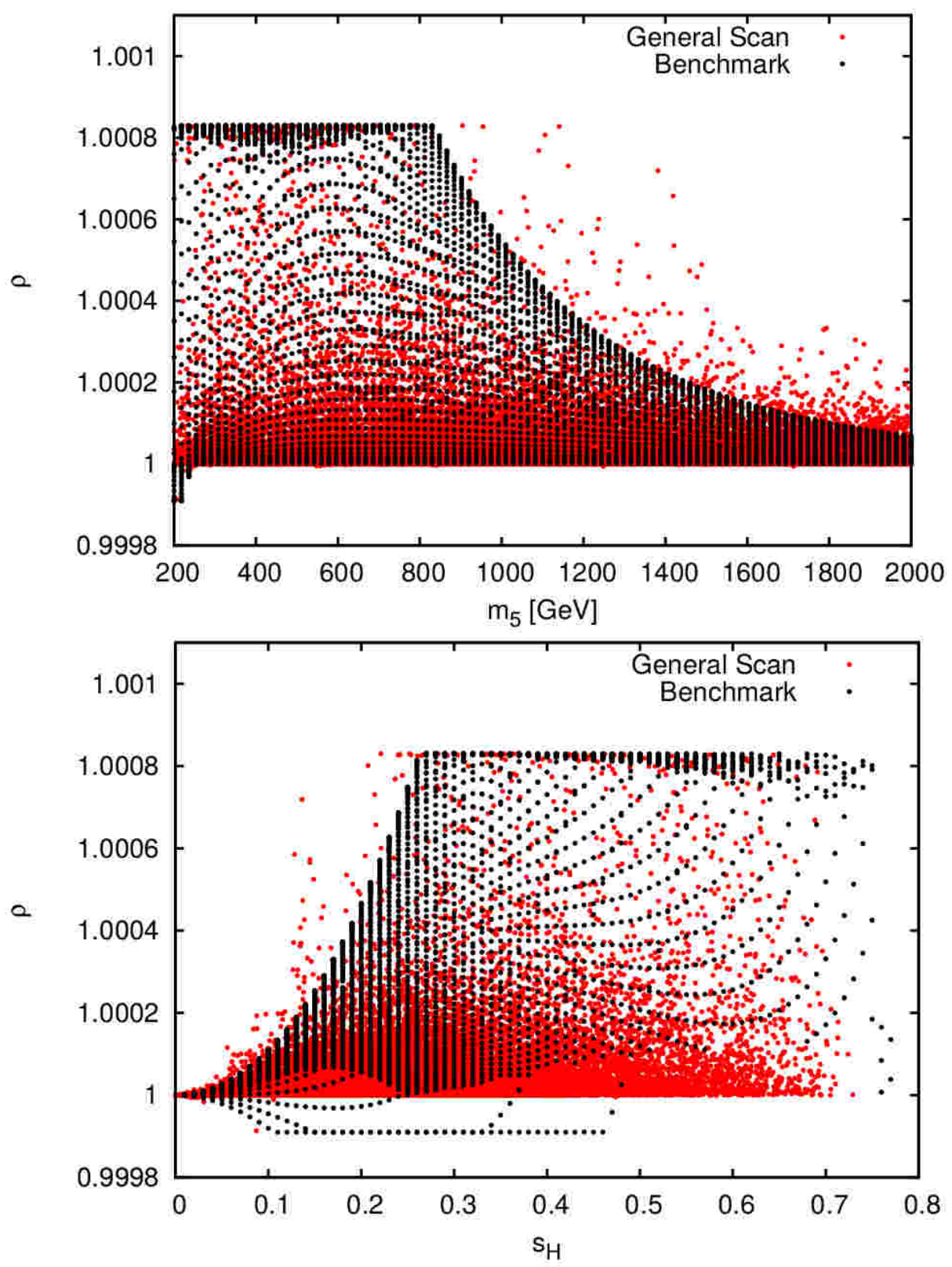

Figure 4.5: The weak-scale $\rho$ parameter evaluated with the cutoff scale at its maximum allowed value in a general scan (red) and in the H5plane benchmark (black), as a function of $m_{5}$ (top) and $s_{H}$ (bottom). Both the general scan and the H5plane benchmark populate the entire $\pm 2 \sigma$ allowed region of $\rho \in(0.99991,1.00083)$. 


\subsubsection{Custodial violation in couplings}

Custodial symmetry violation can modify the phenomenology of the GM model by changing the decay patterns of the physical Higgs bosons. The most experimentallyinteresting manifestations of this are in the ratio of the couplings of the SM-like Higgs boson mass eigenstate $\tilde{h}$ to $W$ boson and $Z$ boson pairs, $\lambda_{W Z}^{\tilde{h}} \equiv \kappa_{W}^{\tilde{h}} / \kappa_{Z}^{\tilde{h}}$ [Eq. (4.55)],

and in the couplings of the otherwise-fermiophobic mass eigenstates $\tilde{H}_{5}^{ \pm}$and $\tilde{H}_{5}^{0}$ to fermion pairs induced by custodial-violating mixing among the custodial-symmetry eigenstates [Eqs. (4.37) and (4.54)]. In what follows we maximize the custodialviolating effects by taking the scale of the custodial-symmetric theory as high as possible, subject to the constraints from perturbative unitarity and the $\rho$ parameter. In what follows, we focus on the H5plane benchmark and its comparison to a general parameter scan. We discuss the dedicated low- $m_{5}$ parameter scan in Sec. 4.3.6.

In Fig. 4.6 we plot the deviation of $\lambda_{W Z}^{\tilde{h}}$ from its SM value of 1 in the H5plane benchmark. The effect is tiny, reaching at most half a percent in a small region of the H5plane benchmark with $m_{5} \lesssim 250 \mathrm{GeV}$ and moderate values of $s_{H}$; for larger $m_{5}$, the deviation is below two per mille. This deviation is well below the sensitivity of the current experimental measurement at the LHC, $\lambda_{W Z}^{\tilde{h}}=0.95 \pm 0.08$ [99]. It is also below the expected sensitivity obtained by combining the projections for the measurement precision of the SM Higgs couplings $\kappa_{W}$ and $\kappa_{Z}$ at the High-Luminosity LHC (a few percent) and the proposed International Linear $e^{+} e^{-}$Collider (ILC) (roughly half a percent) as summarized in Ref. [100]. The proposed Future Circular Collider (FCCee) could begin to reach the required precision, with projected sensitivity for $\kappa_{W}$ and 


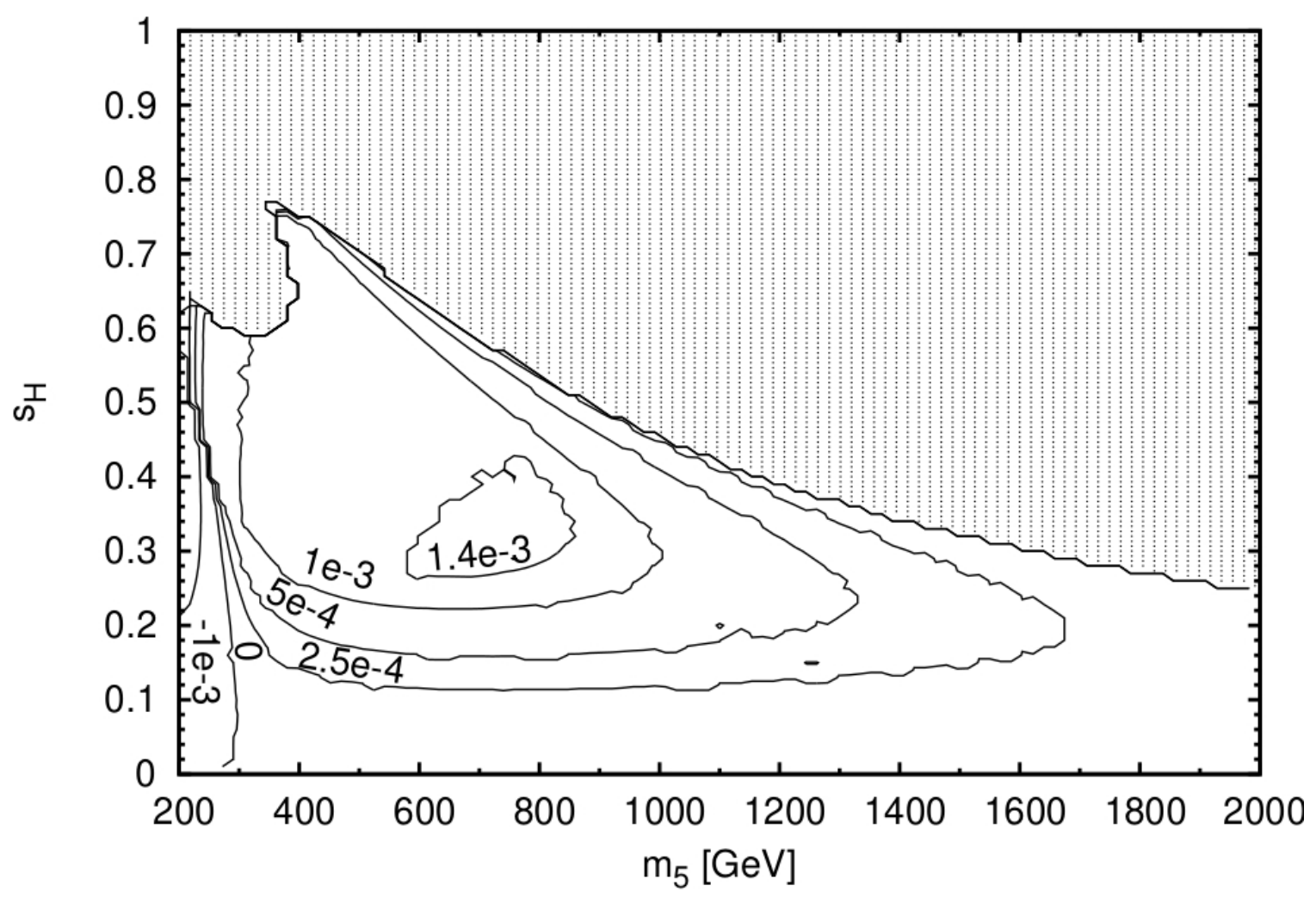

Figure 4.6: Contours of $\delta \lambda_{W Z}^{\tilde{h}} \equiv \lambda_{W Z}^{\tilde{h}}-1$ in the H5plane benchmark, taking the scale of the custodial-symmetric theory to be as large as possible subject to perturbative unitarity and the $\rho$ parameter constraint. $\delta \lambda_{W Z}^{\tilde{h}}$ varies between $-5.1 \times 10^{-3}$ and $1.4 \times 10^{-3}$.

$\kappa_{Z}$ of 1.5 to 2 per mille [101]. ${ }^{3}$

In Fig. 4.7 we compare the value of $\lambda_{W Z}^{\tilde{h}}$ evaluated with the maximum allowed cutoff scale in the H5plane benchmark (black points) to the results of a general parameter scan (red points). The range of deviations in the H5plane benchmark is representative of that in the general scan, except for $m_{5} \simeq 200 \mathrm{GeV}$ and moderate $s_{H}$ in which the H5plane benchmark probes a rather atypical region of parameter space in which negative deviations of up to half a percent are possible. In the general scan

\footnotetext{
${ }^{3}$ Because these coupling extraction methods are based on measurements of Higgs production cross sections and decay branching ratios, they probe only the magnitude of $\lambda_{W Z}^{\tilde{h}}$, not the sign; a method involving the dependence of the $\tilde{h} \rightarrow 4 \ell$ decay distributions on the $\tilde{h} W W$ coupling at one loop provides sensitivity to the sign of $\lambda_{W Z}^{\tilde{h}}$, but can achieve a precision only of order $20-50 \%$ at the High-Luminosity LHC [102].
} 
the deviation is typically below $0.2 \%$.

In Fig. 4.8 we plot the custodial-violation-induced coupling and branching ratio of $\tilde{H}_{5}^{0}$ to fermions in the H5plane benchmark. The $\tilde{H}_{5}^{0}$ coupling to fermions $\kappa_{f}^{\tilde{H}_{5}^{0}}$ reaches a magnitude of at most 0.04 in the H5plane benchmark, leading to fermioninduced (e.g., via gluon fusion) production cross sections at most $(0.04)^{2}=1.6 \times 10^{-3}$ times that of a SM Higgs boson of the same mass. Potentially more interesting is the effect of this coupling on the $\tilde{H}_{5}^{0}$ decays: as shown in the bottom panel of Fig. 4.8, the branching ratio of $\tilde{H}_{5}^{0}$ to fermions can reach almost half a percent in the H5plane benchmark. For $\tilde{H}_{5}^{0}$ masses above $350 \mathrm{GeV}$, these fermionic decays are overwhelmingly into $t \bar{t}$ pairs.

In Fig. 4.9 we compare these results to the range of $\kappa_{f}^{\tilde{H}_{5}^{0}}$ accessible in the general parameter scan. The general scan can yield significantly larger values of this custodialviolation-induced coupling, reaching as high as \pm 0.5 and populating both positive and negative values. The maximum size of the coupling grows with $s_{H}$. In contrast, the H5plane benchmark yields quite small couplings of magnitude at most 0.04 and mainly negative values. The large custodial-symmetry-violating coupling values in the general scan are due to resonant mixing between the $H_{5}^{0}$ and $H$ states when their masses are nearly degenerate. We illustrate this in the top panel of Fig. 4.10, where we plot $\operatorname{BR}\left(\tilde{H}_{5}^{0} \rightarrow f \bar{f}\right)$ as a function of the mass difference between $\tilde{H}_{5}^{0}$ and $\tilde{H}$. In the mass-degenerate region the mixing is enhanced and fermionic branching ratios on the order of $10-20 \%$ are possible. We also show this branching ratio as a function of $m_{5}$ in the bottom panel of Fig. 4.10; the branching ratio to fermions reaches its maximum for $m_{5}$ between 600 and $800 \mathrm{GeV}$ and falls with increasing $m_{5}$.

In Fig. 4.11 we plot the custodial-violation-induced coupling and branching ratio of $\tilde{H}_{5}^{ \pm}$to fermions in the H5plane benchmark. The $\tilde{H}_{5}^{ \pm}$coupling to fermions $\kappa_{f}^{\tilde{H}_{5}^{+}}$ 

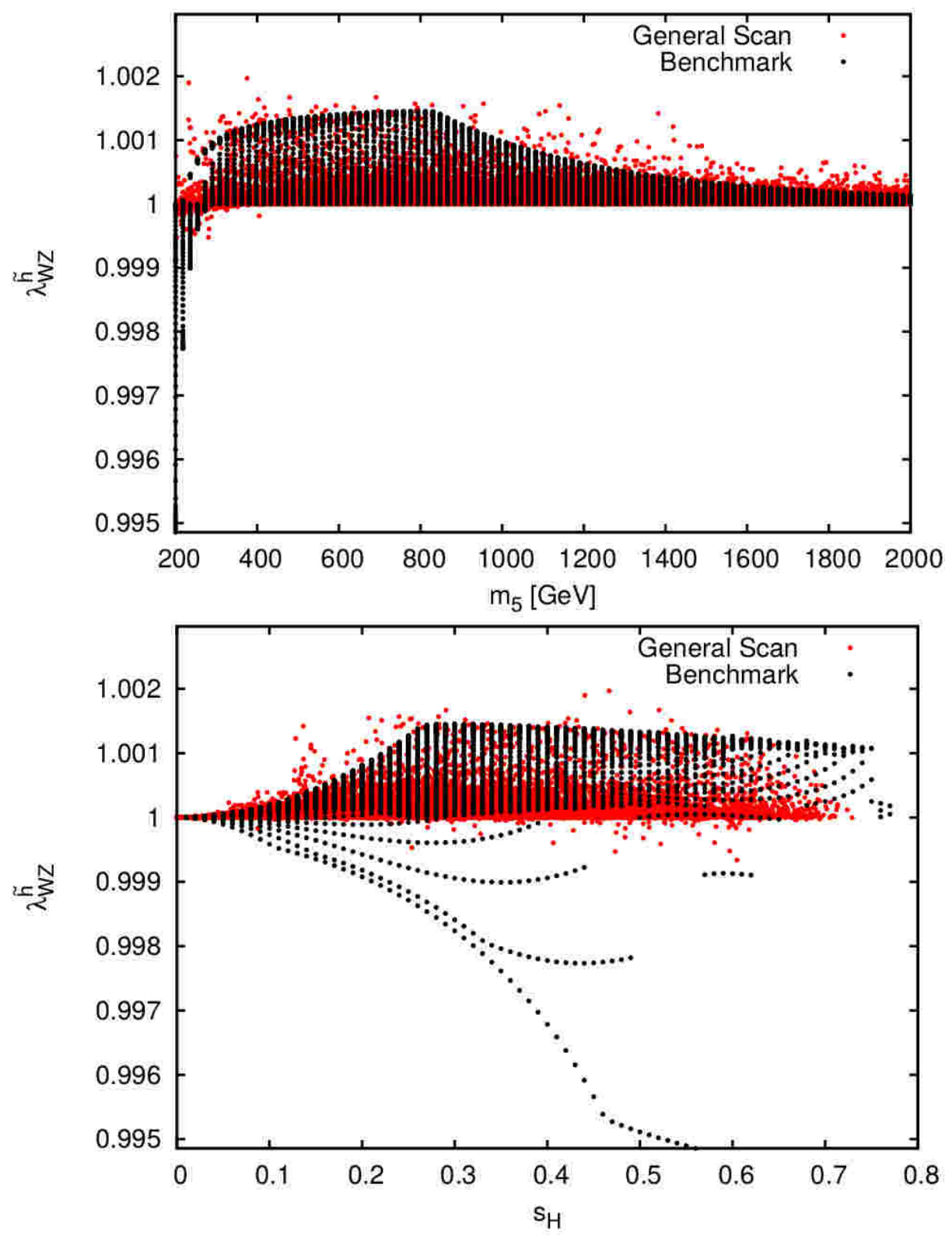

Figure 4.7: $\lambda_{W Z}^{\tilde{h}} \equiv \kappa_{W}^{\tilde{h}} / \kappa_{Z}^{\tilde{h}}$ evaluated with the maximum allowed cutoff scale for a general parameter scan (red) and in the H5plane benchmark (black), as a function of $m_{5}$ (top) and $s_{H}$ (bottom). The minimum value in the general scan is 0.99934 and the maximum value is 1.00197 . 

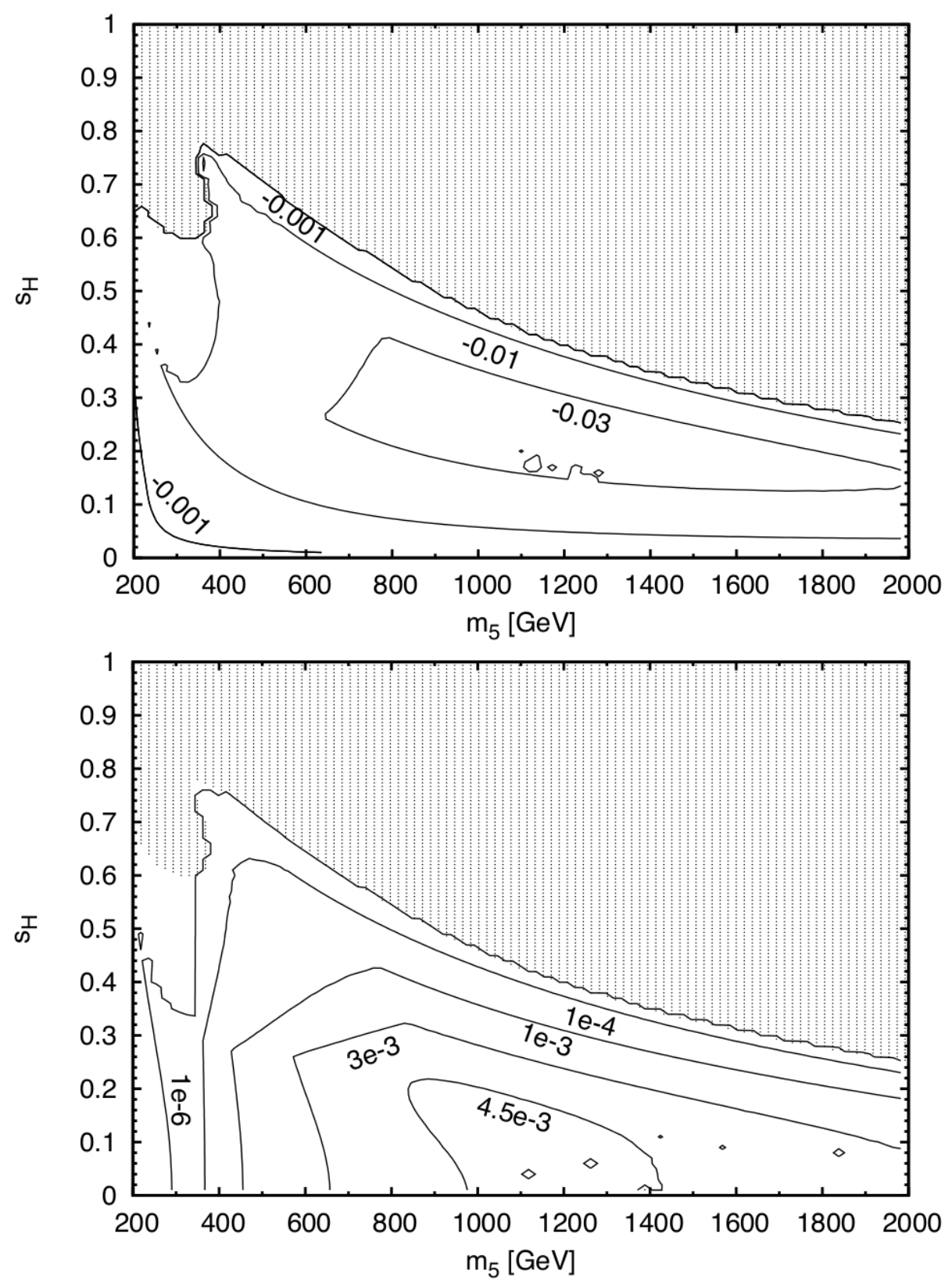

Figure 4.8: The coupling of $\tilde{H}_{5}^{0}$ to fermions and the resulting fermionic branching ratio in the H5plane benchmark, taking the scale of the custodial-symmetric theory to be as large as possible subject to perturbative unitarity and the $\rho$ parameter constraint. Top: contours of $\kappa_{f}^{\tilde{H}_{5}^{0}}$ [defined above Eq. (4.54)]. The allowed values range between $-4.0 \times 10^{-2}$ and $2.0 \times 10^{-3}$. Bottom: contours of the branching ratio of $\tilde{H}_{5}^{0}$ to fermions. We compute only the partial width to the heaviest kinematically accessible pair of fermions; i.e., to $t \bar{t}$ for $m_{\tilde{H}_{5}^{0}}>2 m_{t}$ and $b \bar{b}$ otherwise. The branching ratio of $\tilde{H}_{5}^{0}$ to fermions ranges from $3.5 \times 10^{-11}$ to $4.8 \times 10^{-3}$. 

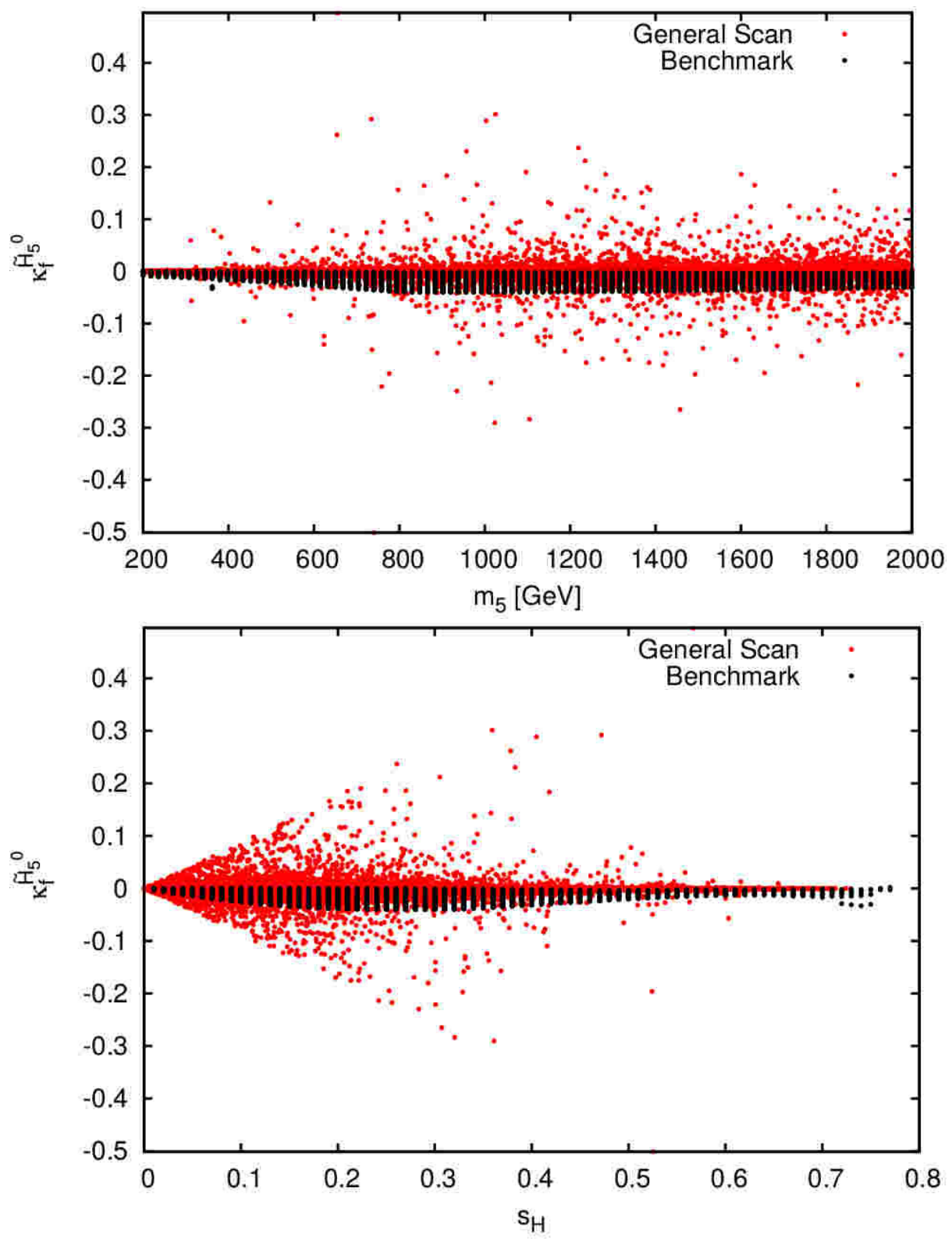

Figure 4.9: The coupling $\kappa_{f}^{\tilde{H}_{5}^{0}}$ of $\tilde{H}_{5}^{0}$ to fermions evaluated with the maximum allowed cutoff scale in a general parameter scan (red) and in the H5plane benchmark (black), as a function of $m_{5}$ (top) and $s_{H}$ (bottom). In the general scan the coupling ranges between -0.50 and +0.50 (for rare points at large $s_{H}$ between 0.5 and 0.6 ). This coupling is zero in the custodial-symmetric model. 

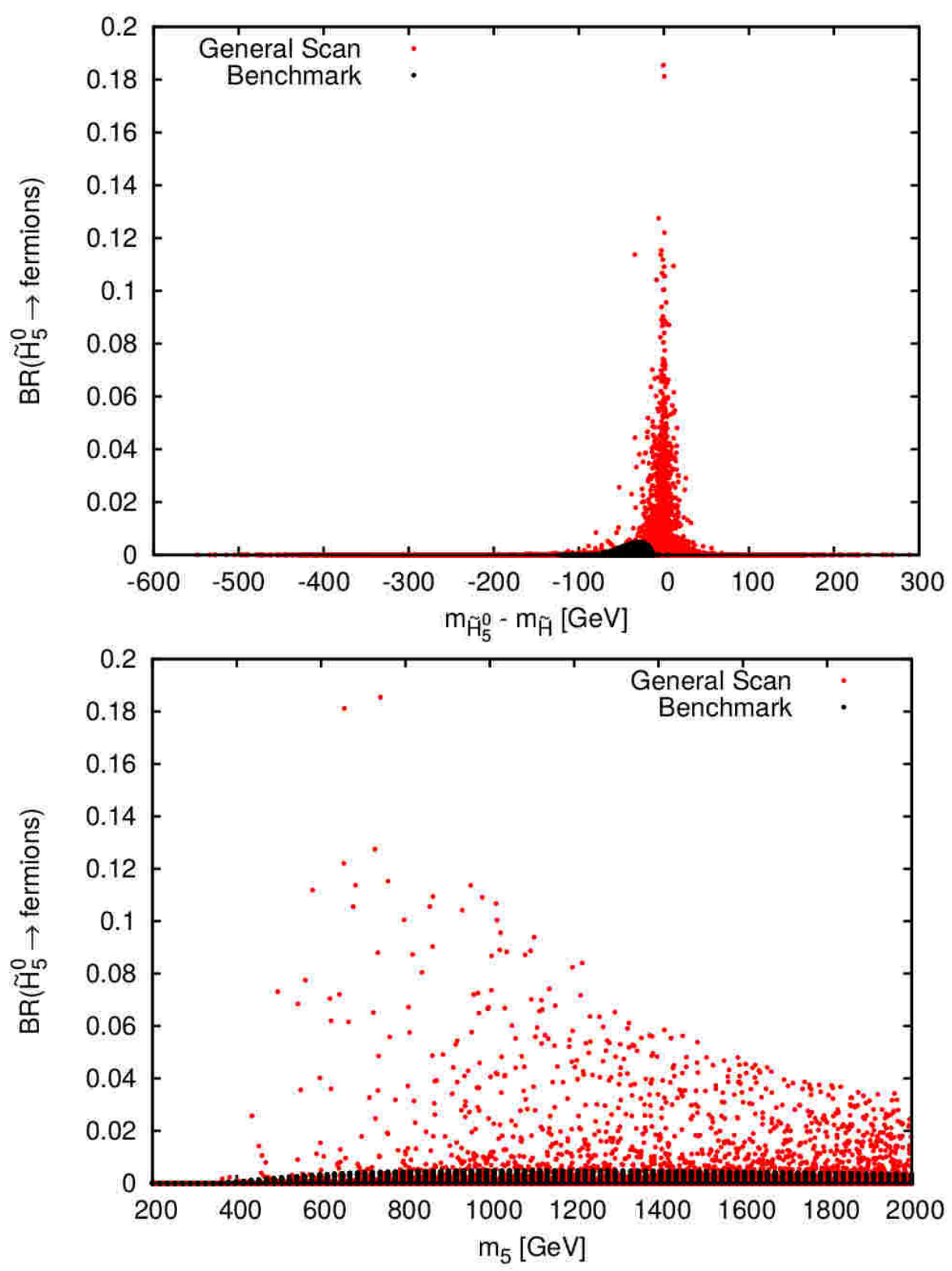

Figure 4.10: Branching ratio of $\tilde{H}_{5}^{0} \rightarrow f \bar{f}$ evaluated with the maximum allowed cutoff scale in a general parameter scan (red) and in the H5plane benchmark (black), as a function of the mass difference $m_{\tilde{H}_{5}^{0}}-m_{\tilde{H}}$ showing the resonant mixing effect (top) and $m_{5}$ (bottom). The maximum branching ratio to fermions in the general scan is $19 \%$. 
reaches a magnitude of at most 0.052 in the $\mathrm{H} 5$ plane benchmark. Again, production processes involving $\tilde{H}_{5}^{+}$coupling to fermions, such as associated production with a top quark, will have cross sections that are far too small to be interesting at the LHC. The branching ratio of $\tilde{H}_{5}^{+} \rightarrow t \bar{b}$ can reach $1.2 \%$, as shown in the right panel of Fig. 4.11.

In Fig. 4.12 we compare these results to the range of $\kappa_{f}^{\tilde{H}_{5}^{+}}$accessible in the general parameter scan. The general scan can again yield larger values of this custodialviolation-induced coupling, reaching a magnitude of at most 0.3 and populating both positive and negative values. The maximum size of the coupling again grows with $s_{H}$. In contrast, the H5plane benchmark yields somewhat smaller values of this coupling of at most 0.052 and only populates positive values. The large custodialsymmetry-violating coupling values that can be obtained in the general scan are again a consequence of resonant mixing, this time between the $H_{5}^{+}$and the $H_{3}^{+}$states when they are nearly degenerate. We illustrate this in the left panel of Fig. 4.13, where we $\operatorname{plot} \operatorname{BR}\left(\tilde{H}_{5}^{+} \rightarrow f \bar{f}^{\prime}\right)$ as a function of the mass difference between $\tilde{H}_{5}^{+}$and $\tilde{H}_{3}^{+}$. In this mass-degenerate region the mixing is enhanced and fermionic branching ratios on the order of $20-30 \%$ are possible. We also show this branching ratio as a function of $m_{5}$ in the bottom panel of Fig. 4.13; the branching ratio to fermions again falls with increasing $m_{5}$.

The custodial-violation-induced decays of $\tilde{H}_{5}^{0}$ and $\tilde{H}_{5}^{ \pm}$to fermion pairs do not dramatically alter the phenomenology within the H5plane benchmark, and do so in the general scans only when there are near mass degeneracies with the fermiophilic heavy Higgs bosons $H$ or $H_{3}^{+}$. Potentially more interesting is the effect of fermionic decays of these particles for low masses below the $W W$ or $W Z$ thresholds, when the dominant diboson decays of these scalars go off shell. In the custodial-symmetric GM 

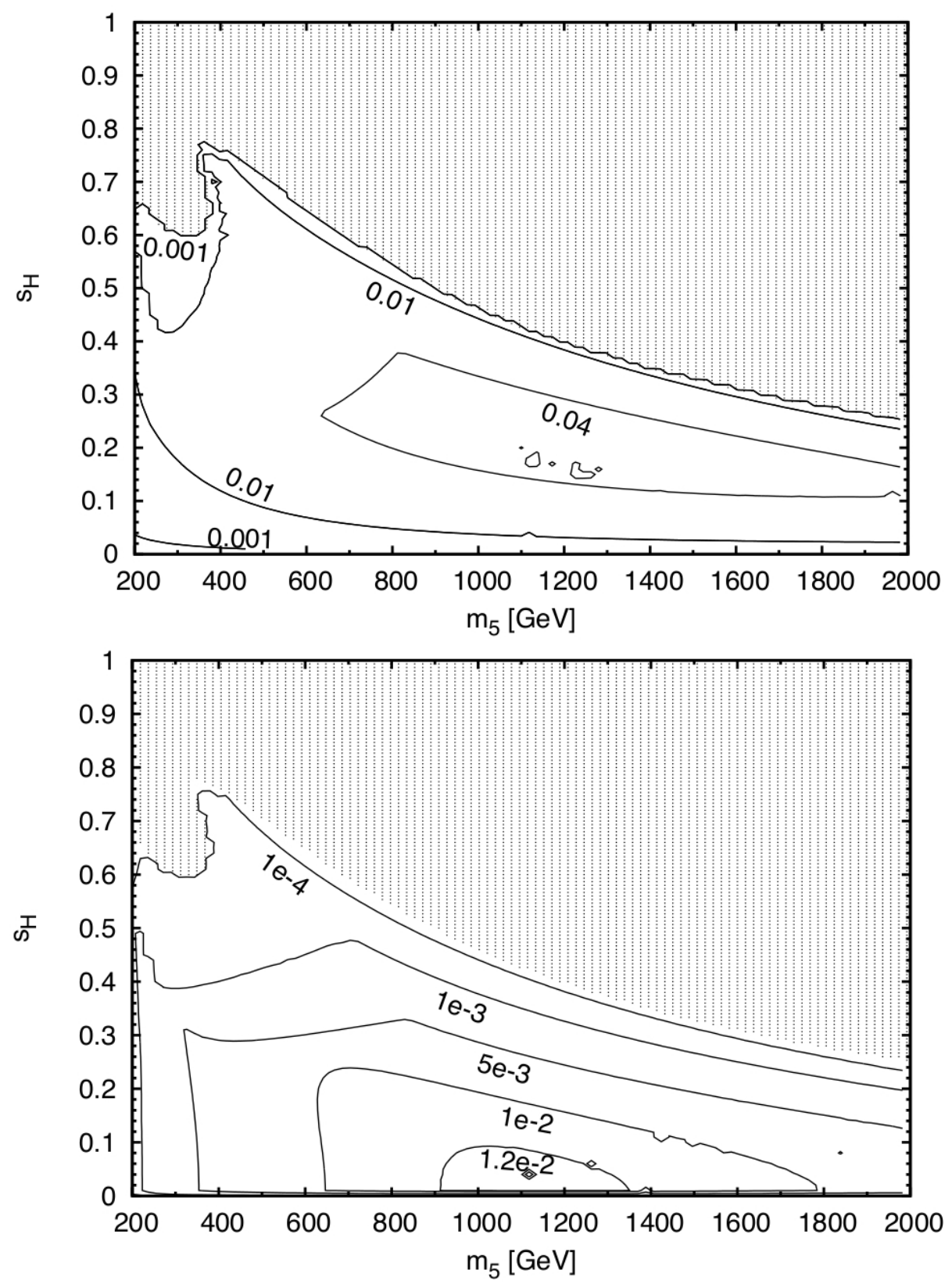

Figure 4.11: The coupling of $\tilde{H}_{5}^{+}$to fermions and the resulting fermionic branching ratio in the H5plane benchmark, taking the scale of the custodial-symmetric theory to be as large as possible subject to perturbative unitarity and the $\rho$ parameter constraint. Top: contours of $\kappa_{f}^{\tilde{H}_{5}^{+}}$[defined in Eq. (4.36)]. The allowed values range between $1.0 \times 10^{-4}$ and $5.2 \times 10^{-2}$. Bottom: contours of the branching ratio of $\tilde{H}_{5}^{+}$to fermions, including only the decay to $t \bar{b}$. This branching ratio ranges from $2.0 \times 10^{-8}$ to $1.2 \times 10^{-2}$. 

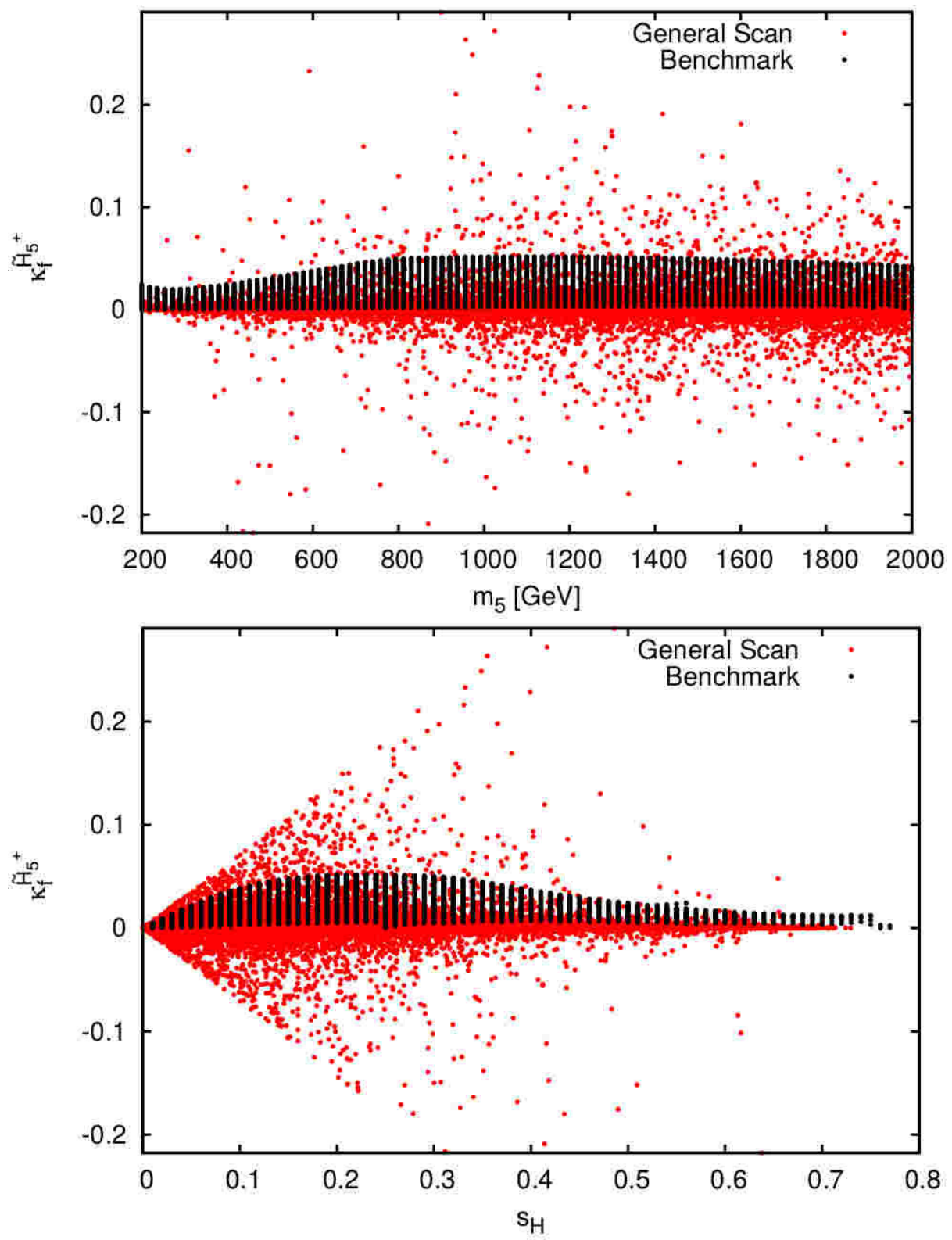

Figure 4.12: The coupling $\kappa_{f}^{\tilde{H}_{5}^{+}}$of $\tilde{H}_{5}^{+}$to fermions evaluated with the maximum allowed cutoff scale in a general parameter scan (red) and in the H5plane benchmark (black), as a function of $m_{5}$ (top) and $s_{H}$ (bottom). In the general scan the coupling ranges between -0.22 and +0.29 . 

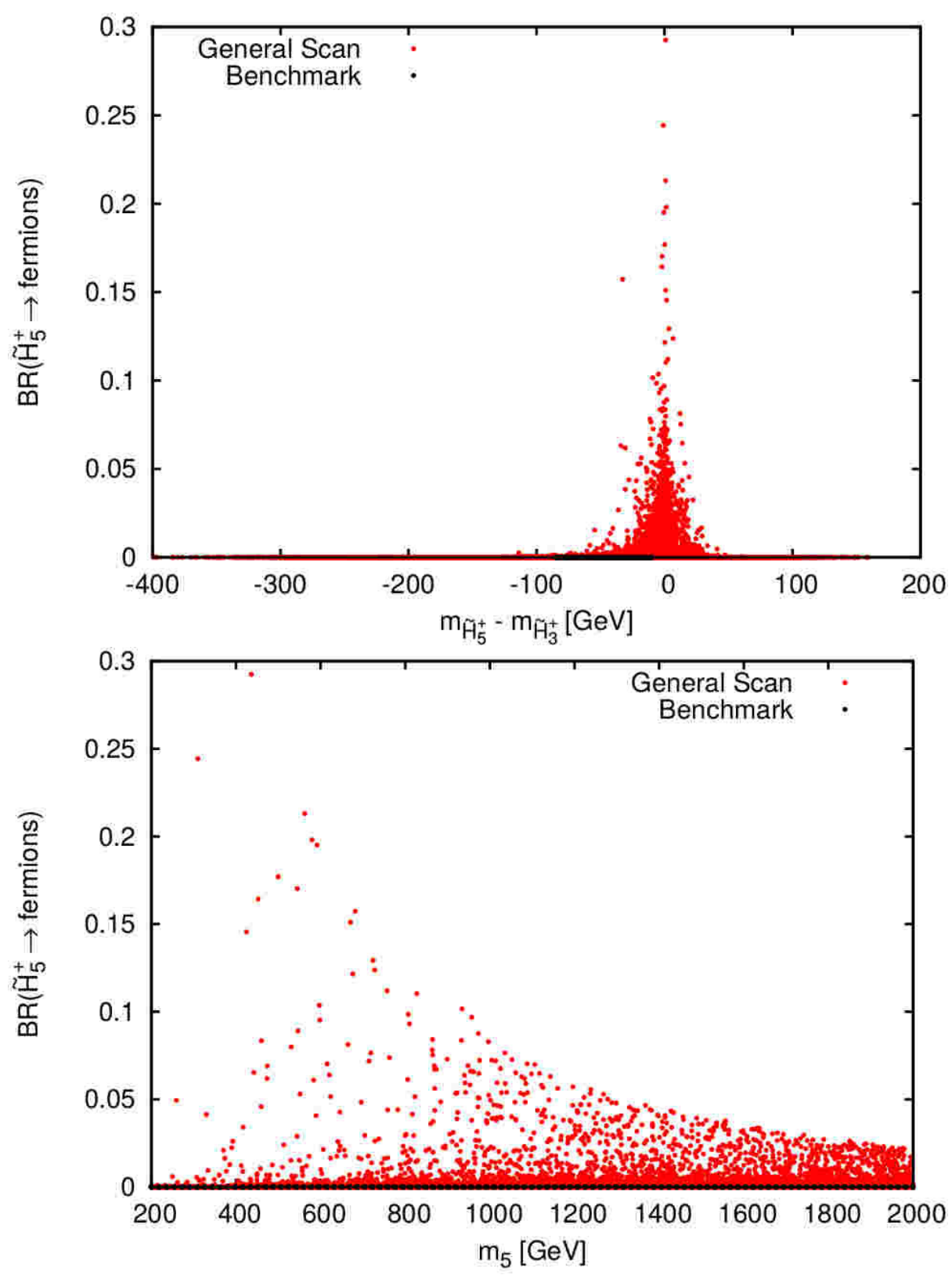

Figure 4.13: Branching ratio of $\tilde{H}_{5}^{+} \rightarrow t \bar{b}$ evaluated with the maximum allowed cutoff scale in a general parameter scan (red) and in the H5plane benchmark (black), as a function of the mass difference $m_{\tilde{H}_{5}^{+}}-m_{\tilde{H}_{3}^{+}}$showing the resonant mixing effect (top) and $m_{5}$ (bottom). The maximum branching ratio to fermions in the general scan is $29 \%$. 
model, $H_{5}^{0}$ decays to $\gamma \gamma$ and $H_{5}^{+}$decays to $W^{+} \gamma$ become interesting for these low masses [94,103, 104]; competition from custodial-violation-induced fermionic decays could dramatically change the phenomenology in this mass region. We perform a detailed study of this low $m_{5}$ region in Sec. 4.3.6.

\subsubsection{Custodial-violating mass splittings}

Custodial symmetry violation also induces splittings between the masses of the otherwisedegenerate custodial fiveplet and triplet states. These splittings follow a universal pattern everywhere within the H5plane benchmark and over the vast majority of the parameter space of our general scans. We again maximize the custodial-violating effects in what follows by taking the scale of the custodial-symmetric theory as high as possible, subject to the constraints from perturbative unitarity and the $\rho$ parameter.

Among the custodial-triplet mass eigenstates, $\tilde{H}_{3}^{0}$ is almost always heavier than $\tilde{H}_{3}^{+}$, and both of these masses are shifted up relative to the weak-scale custodialsymmetric input value of $m_{3}$. The splittings are small, as shown in Fig. 4.14 for the H5plane benchmark: the mass difference between $\tilde{H}_{3}^{0}$ and $\tilde{H}_{3}^{+}$reaches at most $5.3 \mathrm{GeV}$ (top panel of Fig. 4.14). The shift of the $\tilde{H}_{3}^{0}$ mass upward from the input value of $m_{3}$ is shown in the bottom panel of Fig. 4.14, and is at most $9.1 \mathrm{GeV}$. The shift of the $\tilde{H}_{3}^{+}$mass from the input $m_{3}$ value is smaller, reaching at most $3.9 \mathrm{GeV}$ in the benchmark.

In Fig. 4.15 we compare the mass splittings among the custodial triplet states in the H5plane benchmark (black points) to the results of a general scan (red points). In the top panel we plot $m_{\tilde{H}_{3}^{+}}-m_{\tilde{H}_{3}^{0}}$ versus $m_{5}$, and in the bottom panel we plot $m_{\tilde{H}_{3}^{+}}-$ $m_{\tilde{H}_{3}^{0}}$ versus $s_{H}$. The range of mass splittings obtained in the H5plane benchmark is generally typical of the results of the general scan, except that the general scan 

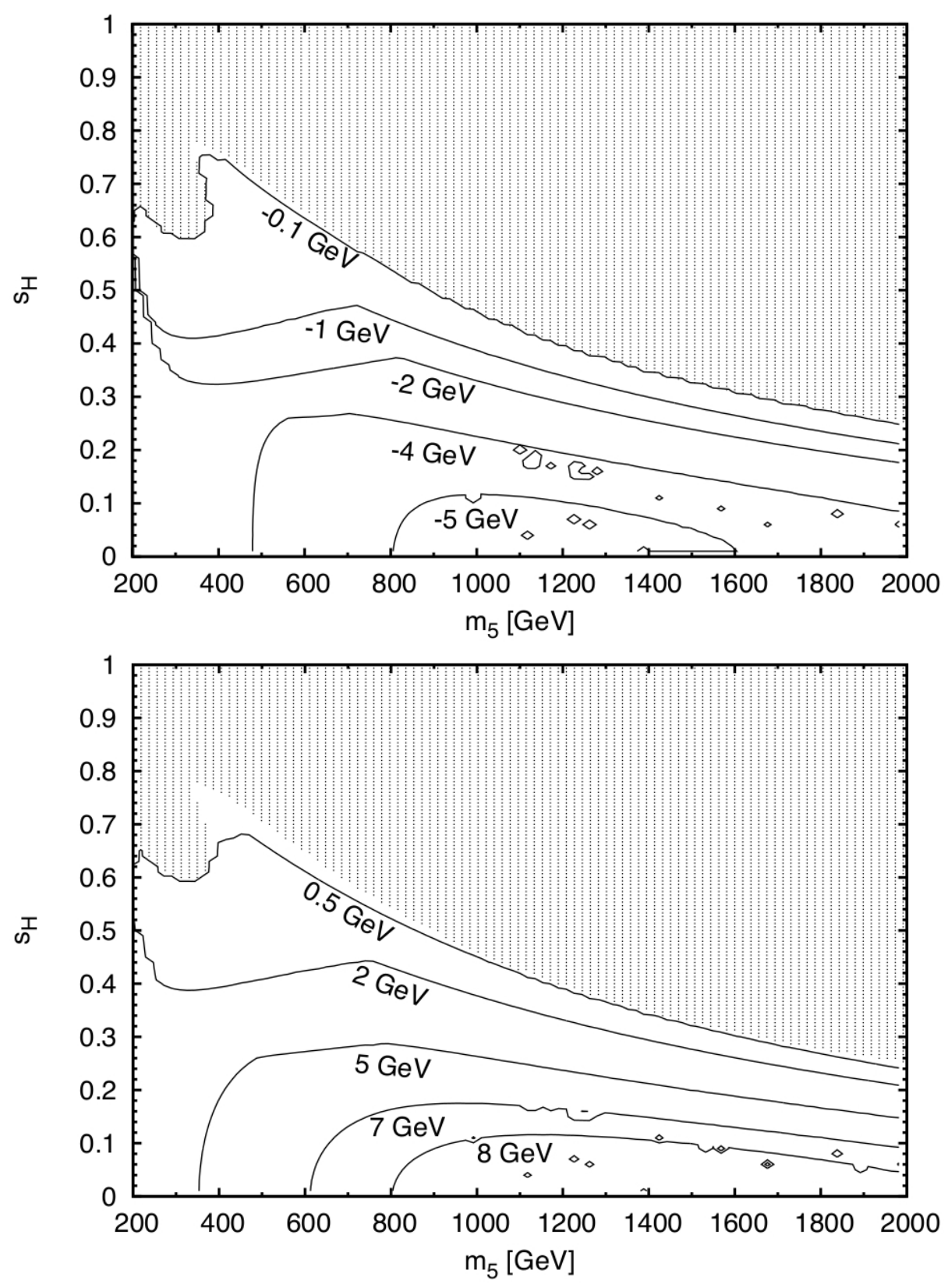

Figure 4.14: The mass splittings within the custodial triplet in the H5plane benchmark, taking the scale of the custodial-symmetric theory to be as large as possible subject to perturbative unitarity and the $\rho$ parameter constraint. Top: $m_{\tilde{H}_{3}^{+}}-m_{\tilde{H}_{3}^{0}}$. This quantity is negative because $\tilde{H}_{3}^{+}$is lighter than $\tilde{H}_{3}^{0}$. The mass splitting ranges between zero and $5.3 \mathrm{GeV}$. Bottom: $m_{\tilde{H}_{3}^{0}}-m_{3}$, where $m_{3}$ is the weak-scale custodialsymmetric input value of the custodial triplet mass. $m_{\tilde{H}_{3}^{0}}$ and $m_{\tilde{H}_{3}^{+}}$are both larger than $m_{3}$ over the entire benchmark. In our numerical scan, the difference between $m_{\tilde{H}_{3}^{0}}$ and $m_{3}$ ranges between $4 \mathrm{MeV}$ and $9.1 \mathrm{GeV}$. 
generates a small number of points with mass splittings up to four times as large as in the benchmark. There are also a very small number of points in the general scan with the opposite mass hierarchy, for which $\tilde{H}_{3}^{+}$becomes heavier than $\tilde{H}_{3}^{0}$ by up to $0.25 \mathrm{GeV}$.

Among the custodial-fiveplet mass eigenstates, $\tilde{H}_{5}^{++}$is almost always the heaviest, followed by $\tilde{H}_{5}^{+}$and then $\tilde{H}_{5}^{0}$. Again the mass splittings are small, as shown in Fig. 4.16 for the H5plane benchmark. The top left panel of Fig. 4.16 shows the mass difference between $\tilde{H}_{5}^{++}$and $\tilde{H}_{5}^{0}$, which is at most $7.2 \mathrm{GeV}$. The mass of $\tilde{H}_{5}^{+}$falls between these two, but closer to the lighter $\tilde{H}_{5}^{0}$ state: the mass difference between $\tilde{H}_{5}^{+}$and $\tilde{H}_{5}^{0}$ reaches at most $1.8 \mathrm{GeV}$, as shown in the top right panel of Fig. 4.16. The mass of $\tilde{H}_{5}^{0}$ remains within $2.3 \mathrm{GeV}$ of the weak-scale custodial-symmetric input value of $m_{5}$, but can be heavier or lighter: this is plotted in the bottom left panel of Fig. 4.16. The mass of $\tilde{H}_{5}^{++}$is always larger than $m_{5}$, with the difference reaching a maximum of $9.0 \mathrm{GeV}$, as shown in the bottom right panel of Fig. 4.16. The smallness of these shifts of the physical $\tilde{H}_{5}$ masses relative to the weak-scale custodial-symmetric input value of $m_{5}$ justifies our use of this input value on the $x$ axis of the plots.

In Fig. 4.17 we compare the mass splittings among the custodial fiveplet states in the H5plane benchmark (black points) to the results of a general scan (red points) as a function of $m_{5}$. We show $m_{\tilde{H}_{5}^{++}}-m_{\tilde{H}_{5}^{0}}$ (top left), $m_{\tilde{H}_{5}^{+}}-m_{\tilde{H}_{5}^{0}}$ (top right), and $m_{\tilde{H}_{5}^{++}}-m_{\tilde{H}_{5}^{+}}$(bottom). Again the ranges of mass splittings obtained in the H5plane benchmark are generally typical of the results in the general scan, except that the general scan generates a small number of points with mass splittings up to six times as large as in the benchmark. There are also a very small number of points in the general scan with the opposite mass hierarchy for which $\tilde{H}_{5}^{+}$becomes lighter than $\tilde{H}_{5}^{0}$ 

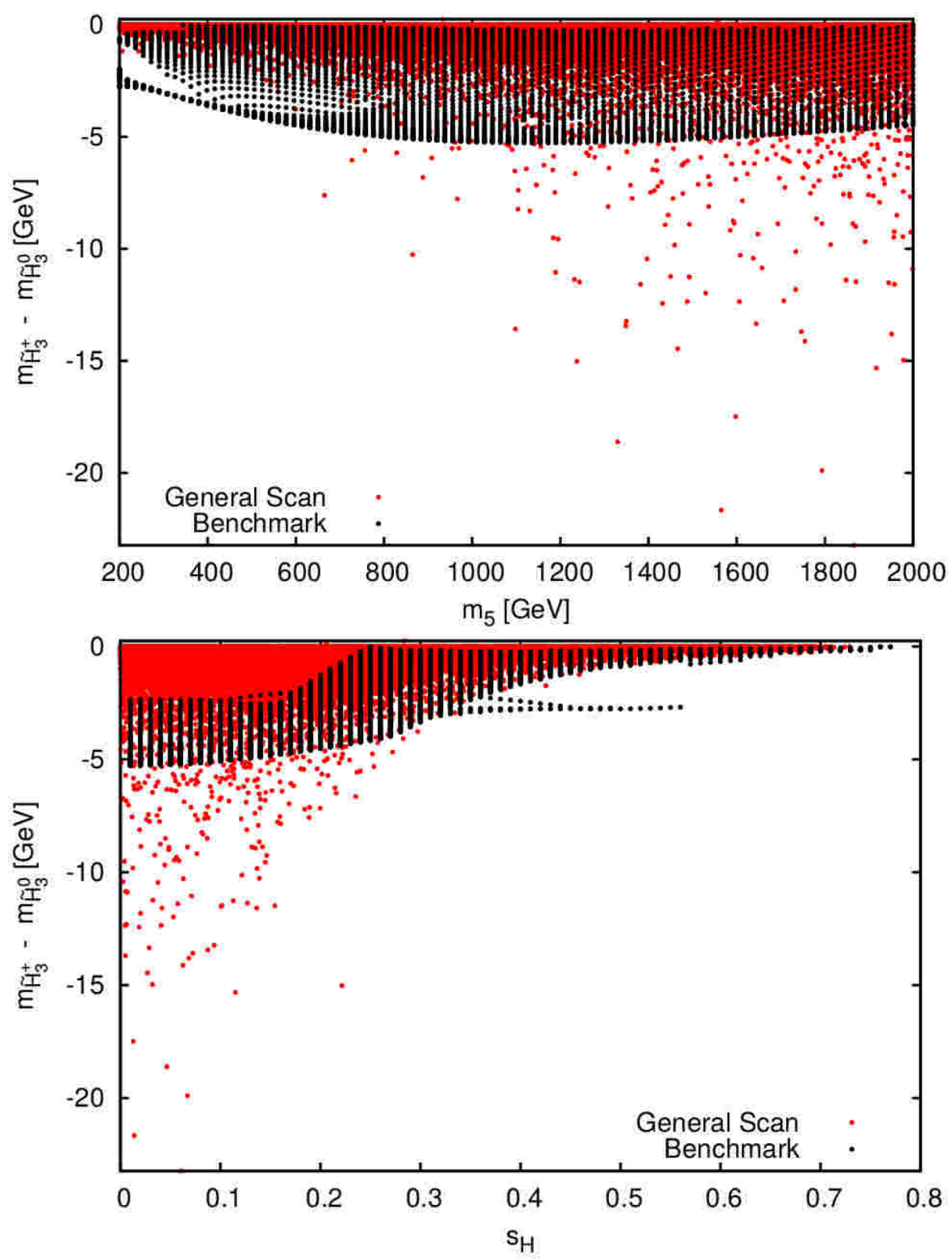

Figure 4.15: Mass splitting $m_{\tilde{H}_{3}^{+}}-m_{\tilde{H}_{3}^{0}}$ evaluated with the maximum allowed cutoff scale in a general parameter scan (red) and in the H5plane benchmark (black), as a function of $m_{5}$ (top) and $s_{H}$ (bottom). This quantity is negative because $\tilde{H}_{3}^{+}$is lighter than $\tilde{H}_{3}^{0}$. The mass splitting in the general scan ranges between $+0.25 \mathrm{GeV}$ and $-23 \mathrm{GeV}$. 

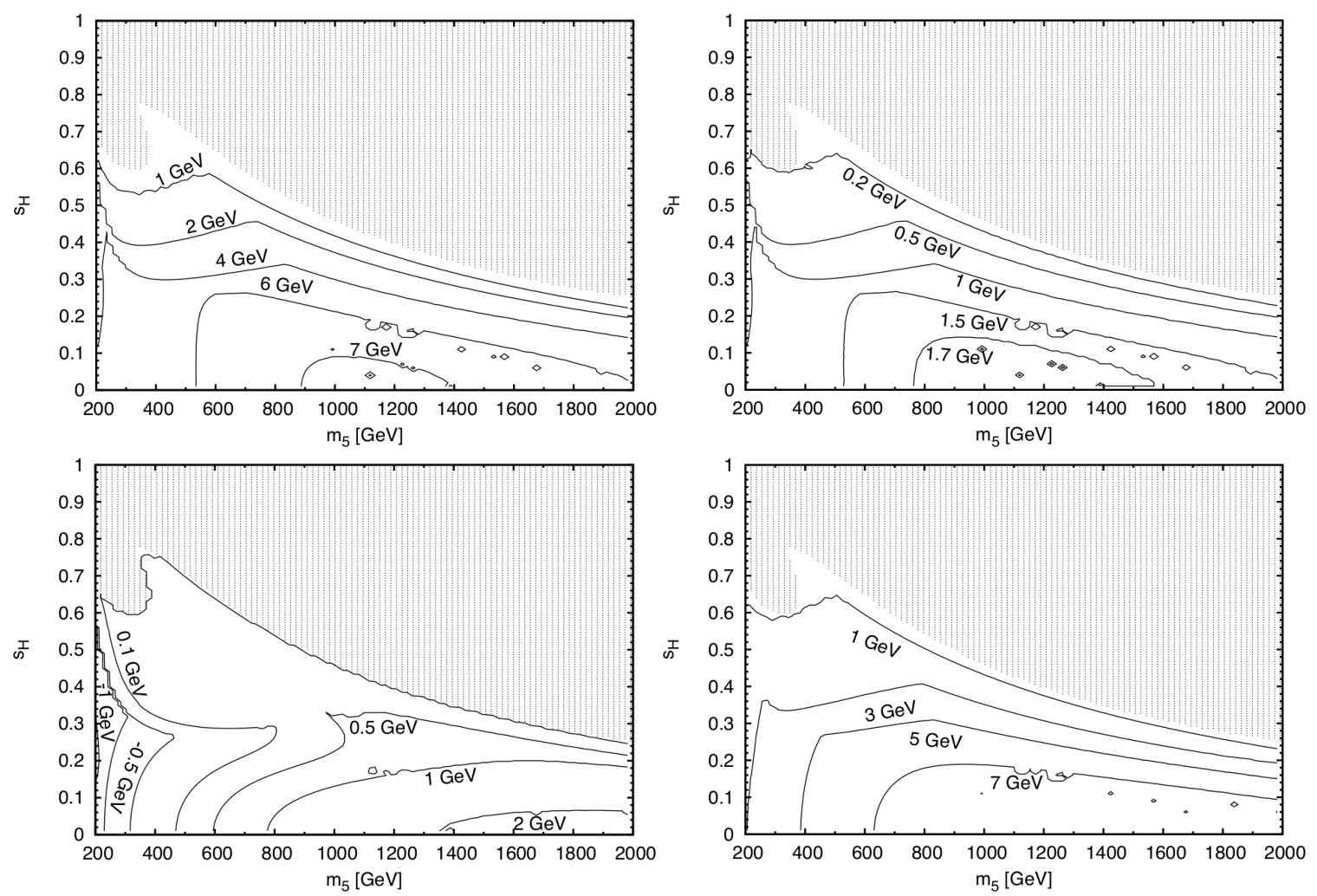

Figure 4.16: The mass splittings within the custodial fiveplet in the H5plane benchmark, taking the scale of the custodial-symmetric theory to be as large as possible subject to perturbative unitarity and the $\rho$ parameter constraint. Top left: $m_{\tilde{H}_{5}^{++}}-m_{\tilde{H}_{5}^{0}}$. This mass splitting ranges between $4.0 \mathrm{MeV}$ and $7.2 \mathrm{GeV}$. Top right: $m_{\tilde{H}_{5}^{+}}-m_{\tilde{H}_{5}^{0}}$. This mass splitting ranges between $6.0 \mathrm{MeV}$ and $1.8 \mathrm{GeV}$. Bottom left: $m_{\tilde{H}_{5}^{0}}-m_{5}$, where $m_{5}$ is the weak-scale custodial-symmetric input value of the custodial fiveplet mass. This mass difference ranges between $-1.5 \mathrm{GeV}$ and $2.3 \mathrm{GeV}$. Bottom right: $m_{\tilde{H}_{5}^{++}}-m_{5} \cdot m_{\tilde{H}_{5}^{++}}$is always larger than $m_{5}$, with the difference ranging between $7.0 \mathrm{MeV}$ and $9.0 \mathrm{GeV}$. 

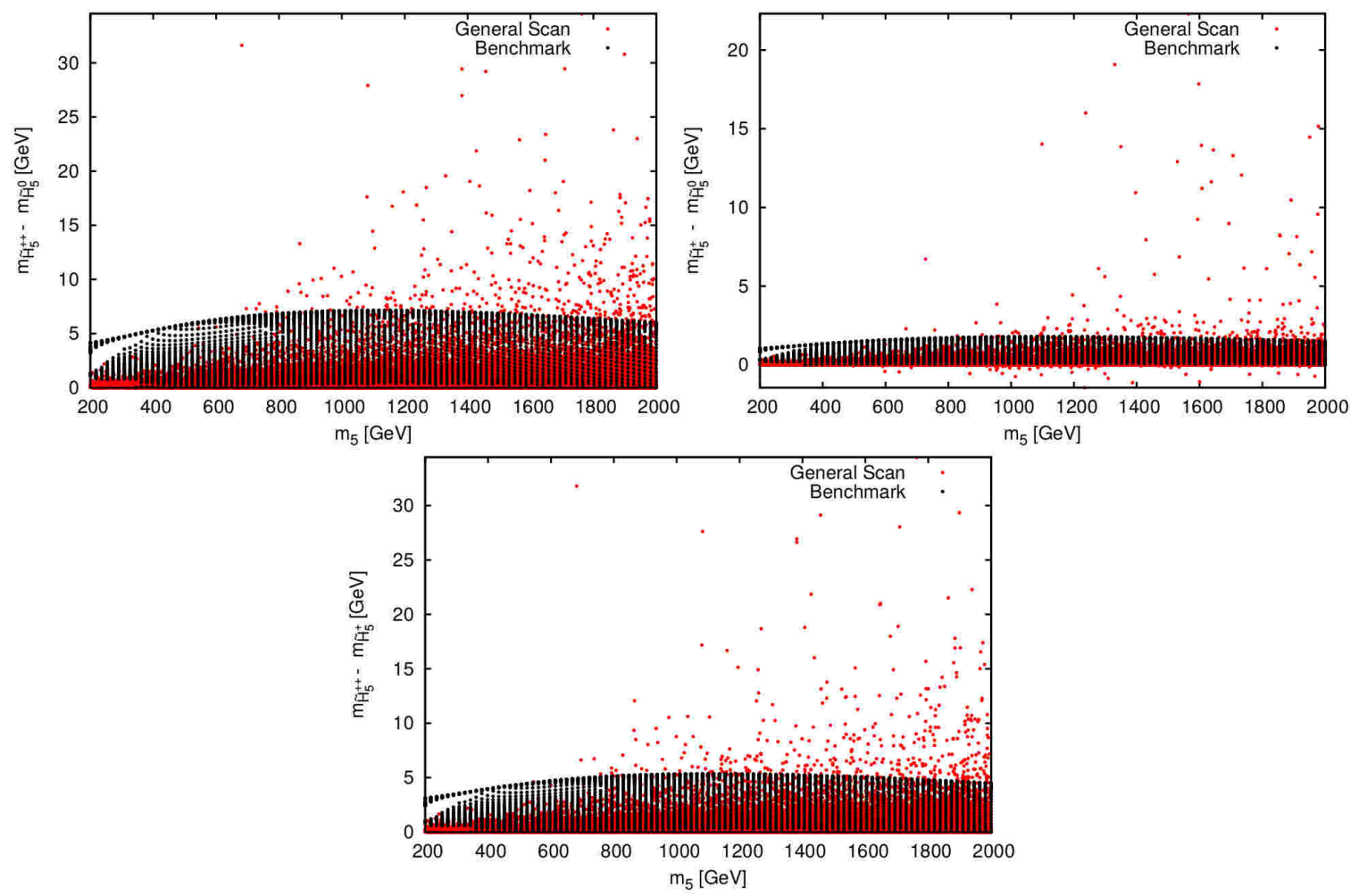

Figure 4.17: Mass splittings $m_{\tilde{H}_{5}^{++}}-m_{\tilde{H}_{5}^{0}}$ (top left), $m_{\tilde{H}_{5}^{+}}-m_{\tilde{H}_{5}^{0}}$ (top right), and $m_{\tilde{H}_{5}^{++}}-m_{\tilde{H}_{5}^{+}}$(bottom) as a function of $m_{5}$, evaluated with the maximum allowed cutoff scale in a general parameter scan (red) and in the H5plane benchmark (black). In the general scan $m_{\tilde{H}_{5}^{++}}-m_{\tilde{H}_{5}^{0}}$ ranges between zero and $34.5 \mathrm{GeV}, m_{\tilde{H}_{5}^{+}}-m_{\tilde{H}_{5}^{0}}$ ranges between $-1.45 \mathrm{GeV}$ and $22.3 \mathrm{GeV}$, and $m_{\tilde{H}_{5}^{++}}-m_{\tilde{H}_{5}^{+}}$ranges between zero and $34.4 \mathrm{GeV}$.

by up to $1.5 \mathrm{GeV}$. It is also possible in the general scan to have a large mass spitting between the $\tilde{H}_{5}^{++}$and $\tilde{H}_{5}^{0}$ but a small mass splitting between $\tilde{H}_{5}^{+}$and the $\tilde{H}_{5}^{0}$.

Examining the contours in Figs. 4.14 and 4.16, it is apparent that within the H5plane benchmark the mass splittings within the fiveplet and within the triplet tend to follow a common pattern albeit with different scaling. In particular, the splitting $m_{\tilde{H}_{5}^{++}}-m_{\tilde{H}_{5}^{0}}$ is very close to four times that of $m_{\tilde{H}_{5}^{+}}-m_{\tilde{H}_{5}^{0}}$. To understand this behavior we expand the mass splittings to first order in the custodial violation, such 
that $\tilde{x}=x+\delta_{x}$. The mass splittings become:

$$
\begin{aligned}
m_{\tilde{H}_{5}^{++}}-m_{\tilde{H}_{5}^{0}=} & \frac{1}{2 m_{5}}\left[\left(v_{\phi}^{2} d_{2}+v_{\phi}^{2} \frac{1}{6 v_{\chi}} d_{3}+v_{\phi}^{2} \frac{M_{1}}{6 v_{\chi}^{2}} d_{1}-v_{\chi} \frac{32}{3} \lambda_{3} d_{1}+16 M_{2} d_{1}\right)\right. \\
& \left.+\frac{2 v_{\phi}^{2}}{3 v_{\chi}} \lambda_{5} d_{1}+v_{\chi}^{2} d_{4}\right], \\
m_{\tilde{H}_{5}^{+}}-m_{\tilde{H}_{5}^{0}}= & \frac{1}{2 m_{5}}\left[\frac{1}{4}\left(v_{\phi}^{2} d_{2}+v_{\phi}^{2} \frac{1}{6 v_{\chi}} d_{3}+v_{\phi}^{2} \frac{M_{1}}{6 v_{\chi}^{2}} d_{1}-v_{\chi} \frac{32}{3} \lambda_{3} d_{1}+16 M_{2} d_{1}\right)\right. \\
& \left.-\frac{v_{\phi}^{2}}{6 v_{\chi}} \lambda_{5} d_{1}+v_{\chi}^{2} d_{4}^{\prime}\right], \\
m_{\tilde{H}_{3}^{+}-m_{\tilde{H}_{3}^{0}}=} & \frac{1}{2 m_{3}}\left[\frac{v^{2}}{4} d_{2}-\frac{v^{2}}{8} d_{3}\right. \\
& \left.+d_{1}\left(-\frac{v^{2}}{v_{\chi}}\left(\frac{\lambda_{5}}{2}+\frac{M_{1}}{8 v_{\chi}}\right)+2 M_{1}+\frac{\lambda_{5}}{4 v_{\chi}}\left(16 v_{\chi}^{2}-v^{2}\right)\right)\right]
\end{aligned}
$$

where $d_{1}$ through $d_{4}$ and $d_{4}^{\prime}$ are zero in the limit of exact custodial symmetry and are given by

$$
\begin{aligned}
& d_{1}=\tilde{v}_{\xi}-\tilde{v}_{\chi}=\frac{v^{2}}{4 v_{\chi}} \Delta \rho \\
& d_{2}=\frac{\tilde{\lambda}_{4}}{\sqrt{2}}-\frac{\tilde{\lambda}_{3}}{2} \\
& d_{3}=\tilde{M}_{1}^{\prime}-\tilde{M}_{1}, \\
& d_{4}=4 \tilde{\lambda}_{2}-\frac{4}{3} \tilde{\lambda}_{7}-\frac{16}{3} \tilde{\lambda}_{8}+\frac{8}{3} \tilde{\lambda}_{10}, \\
& d_{4}^{\prime}=2 \tilde{\lambda}_{9}-\frac{4}{3} \tilde{\lambda}_{7}-\frac{16}{3} \tilde{\lambda}_{8}+\frac{8}{3} \tilde{\lambda}_{10} .
\end{aligned}
$$

The approximate relation $m_{\tilde{H}_{5}^{++}}-m_{\tilde{H}_{5}^{0}} \simeq 4\left(m_{\tilde{H}_{5}^{+}}-m_{\tilde{H}_{5}^{0}}\right)$ is therefore to be expected because only the last two terms in Eqs. (4.61) and (4.62) break this proportionality. Simply from the generic size of the dimensionful parameters in the terms that break this relation, we naively expect that they will be sub-dominant contributors to the 
mass splittings. In the general scan shown in Fig. 4.17 this relation tends to hold to a good approximation throughout the parameter space but can be badly broken by the enhanced mixing caused by approximately degenerate charged eigenstates.

The similarities in the patterns of the fiveplet and triplet mass splittings can also be explained by comparing their approximate forms, as they both depend on the same terms as the sources of custodial violation. Although these terms come in with different coefficients, when a single term dominates one expression, it will generally dominate all of them. In the case of the custodial triplet, the splitting is always negative because the dominant terms (mainly the term proportional to $d_{3}$, but the term proportional to $d_{1}$ is usually significant and sometimes dominant) tend not to change sign throughout the whole parameter space. This remains true for the general parameter scan shown in Fig. 4.15 where the triplet mass splittings are overwhelmingly negative even when $\rho$ is less than 1 .

In Fig. 4.18 we plot the shift of the mass of the physical mass eigenstate $\tilde{H}$ relative to the weak-scale custodial-symmetric input value of $m_{H}$. The $\tilde{H}$ mass is shifted upwards over almost all of the H5plane benchmark, and the shift is by at most 5.6 GeV. We conclude that, within the H5plane benchmark and even allowing for custodial symmetry violation, the custodial-symmetric predictions for the masses of the scalars in the model are reliable to within better than $10 \mathrm{GeV}$.

Experimentally checking the mass degeneracy of the scalars within the custodial triplet and the custodial fiveplet has been proposed as a way to test the custodial symmetry in the GM model $[105,106]$. At the LHC, mass reconstruction of the $H_{3}$ states relies on their decays to dijets, $H_{3}^{+} \rightarrow c \bar{s}, H_{3}^{0} \rightarrow b \bar{b}$ [105]. Considering that the dijet invariant mass resolution at the LHC is not sufficient to kinematically separate the hadronic decays of the $W$ and the $Z$ with their $11 \mathrm{GeV}$ mass difference, it 


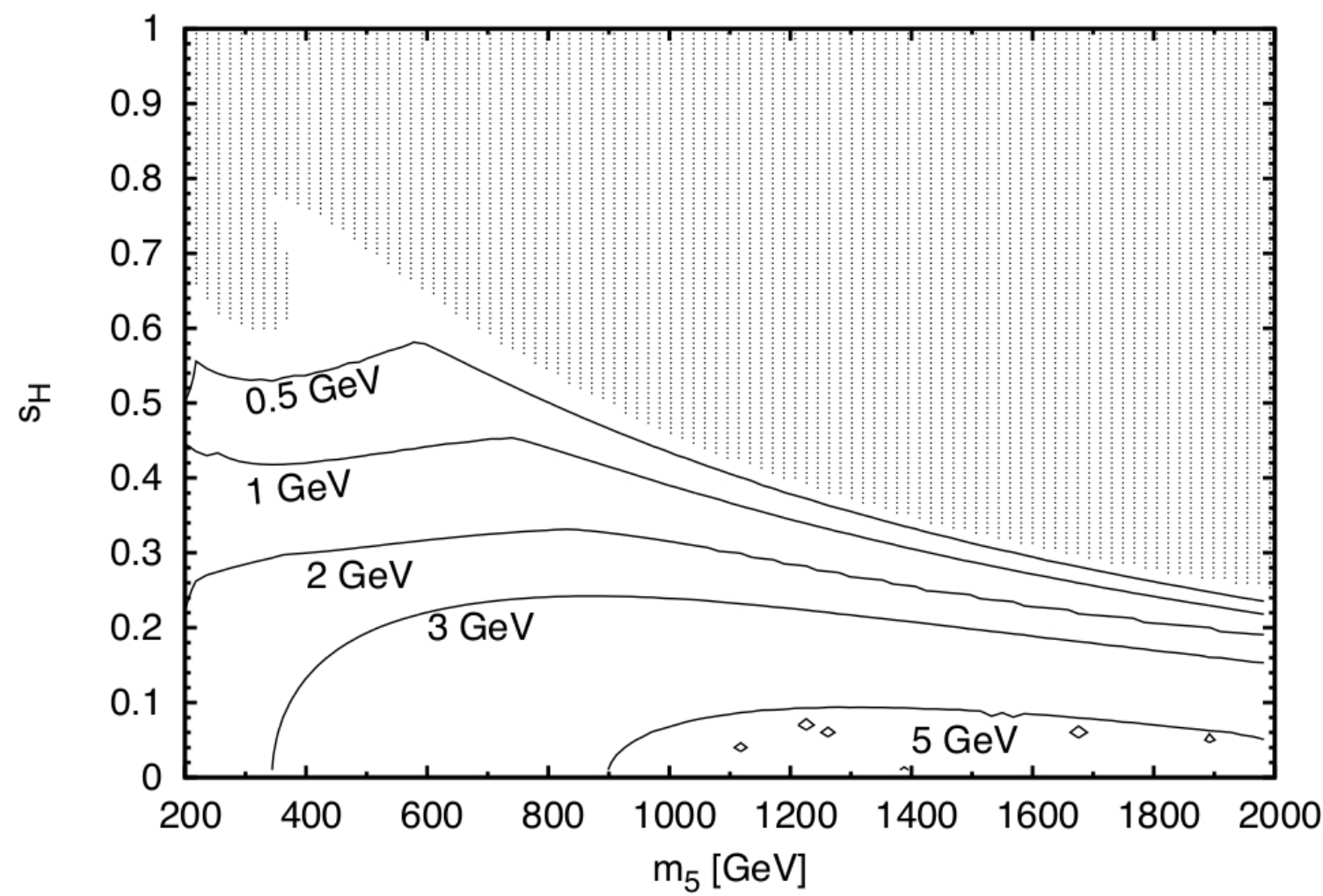

Figure 4.18: Deviation $m_{\tilde{H}}-m_{H}$ of the physical $\tilde{H}$ mass from the mass of the heavier custodial singlet $H$ in the weak-scale custodial-symmetric theory, computed in the H5plane benchmark taking the scale of the custodial-symmetric theory to be as large as possible subject to perturbative unitarity and the $\rho$ parameter constraint. This mass difference ranges between $-0.059 \mathrm{GeV}$ and $+5.6 \mathrm{GeV}$. 
will not be possible to resolve a custodial-symmetry-violation-induced mass splitting between $\tilde{H}_{3}^{+}$and $\tilde{H}_{3}^{0}$ of at most $5.3 \mathrm{GeV}$ within the H5plane benchmark. Mass reconstruction of the $H_{5}$ states at the LHC relies on their decays to vector boson pairs $V V$. Reference [105] studied the fully-leptonic final states, in which the masses of $H_{5}^{++}, H_{5}^{+}$, and $H_{5}^{0}$ could be determined from the endpoint of the transverse mass distribution of the $V V$ final state. The resolution is worse than for a dijet resonance. The ATLAS experiment has performed a search for $H_{5}^{+} \rightarrow W^{+} Z \rightarrow j j \ell^{+} \ell^{-}$[14], in which reconstruction of a mass peak for $\tilde{H}_{5}^{+}$becomes possible; however, the mass resolution is still limited by the dijet invariant mass resolution of the LHC, which is too poor to resolve the custodial-symmetry-violation-induced mass splitting among the $\tilde{H}_{5}$ states spanning at most $7.2 \mathrm{GeV}$ in the H5plane benchmark. Larger mass splittings are possible for a small number of points in the general scan, but these tend to appear at relatively large $m_{5}$ values so that the splittings remain below a few percent of the overall scalar masses, smaller than the single jet energy resolution of the LHC experiments at these energies (see, e.g., Ref. [107]).

Prospects are somewhat better at the ILC, as studied in Ref. [106]. $\tilde{H}_{5}^{0}$ and $\tilde{H}_{5}^{ \pm}$ can be singly produced in $e^{+} e^{-}$collisions via vector boson fusion, or in association with a $Z$ or $W^{\mp}$ boson, respectively. In the clean lepton collider environment, the $H_{5}$ decays to dibosons can be reconstructed using the fully hadronic final states. With the ILC target dijet energy resolution of $\sigma_{E}=0.3 \times \sqrt{E_{j j}} \mathrm{GeV}$ [108], the dijet resolution will be $\sigma_{E} \simeq 3 \mathrm{GeV}$ for $E_{j j} \simeq 100 \mathrm{GeV}$, famously allowing for $W$ and $Z$ bosons to be distinguished in the all-hadronic channel. Unfortunately, even this excellent mass resolution is too poor to resolve the custodial-symmetryviolation-induced mass splitting between $\tilde{H}_{5}^{+}$and $\tilde{H}_{5}^{0}$, which reaches at most $1.8 \mathrm{GeV}$ in the H5plane benchmark. One could hope to do better by using the leptonic decays 
of $H_{5}^{0} \rightarrow Z Z \rightarrow 4 \ell$ and $H_{5}^{ \pm} \rightarrow W^{ \pm} Z \rightarrow \ell^{ \pm} E_{T}^{\text {miss }} \ell^{+} \ell^{-}$; these suffer from smaller branching fractions, but may offer good enough mass resolution to detect the mass splitting effect of the custodial symmetry violation.

\subsubsection{Direct search constraints}

The most stringent direct search constraint on the custodial-symmetric H5plane benchmark comes from a CMS search for $H_{5}^{ \pm \pm}$produced in vector boson fusion and decaying to $W^{ \pm} W^{ \pm} \rightarrow \ell^{ \pm} \ell^{ \pm} E_{T}^{\text {miss }}$ [16]. This constraint excludes $s_{H}$ above 0.2 for $m_{5}=200 \mathrm{GeV}$, rising to $s_{H}=0.45$ at $m_{5}=1000 \mathrm{GeV}$. It is shown in the bottom left panel of Fig. 3.1 in Sec. 3.3.2. We can apply this straightforwardly to the model with custodial symmetry violation by noting the following. First, as shown in the bottom right panel of Fig. 4.16, the physical mass of $\tilde{H}_{5}^{++}$is at most $5 \mathrm{GeV}$ higher than $m_{5}$ in the region of interest in the H5plane benchmark (and not much different in the general scan). Second, we show in Figs. 4.19 and 4.20 the shift in $\tilde{v}_{\chi}$, which controls the $\tilde{H}_{5}^{ \pm \pm} W^{\mp} W^{\mp}$ coupling and hence the vector boson fusion production cross section, relative to the value of $v_{\chi}$ in the weak-scale custodial-symmetric theory. In the H5plane benchmark this shift is negative and amounts to less than a percent, so that the cross section is suppressed by no more than $2 \%$ due to the custodial symmetry violation. In the general scan this conclusion holds for $s_{H}$ values above 0.1 of interest to us here. Finally, the custodial-symmetry-violation-induced mass splitting between $\tilde{H}_{5}^{++}$and $\tilde{H}_{5}^{+}$is less than $5 \mathrm{GeV}$ in the region of interest, too small for the cascade

decay $\tilde{H}_{5}^{ \pm \pm} \rightarrow W^{ \pm} \tilde{H}_{5}^{ \pm}$to compete significantly with the dominant $\tilde{H}_{5}^{ \pm \pm} \rightarrow W^{ \pm} W^{ \pm}$ signal channel. Thus we conclude that this direct search constraint on the custodial symmetry violating parameter space studied in this thesis will be almost identical to that in the custodial-symmetric H5plane benchmark. 


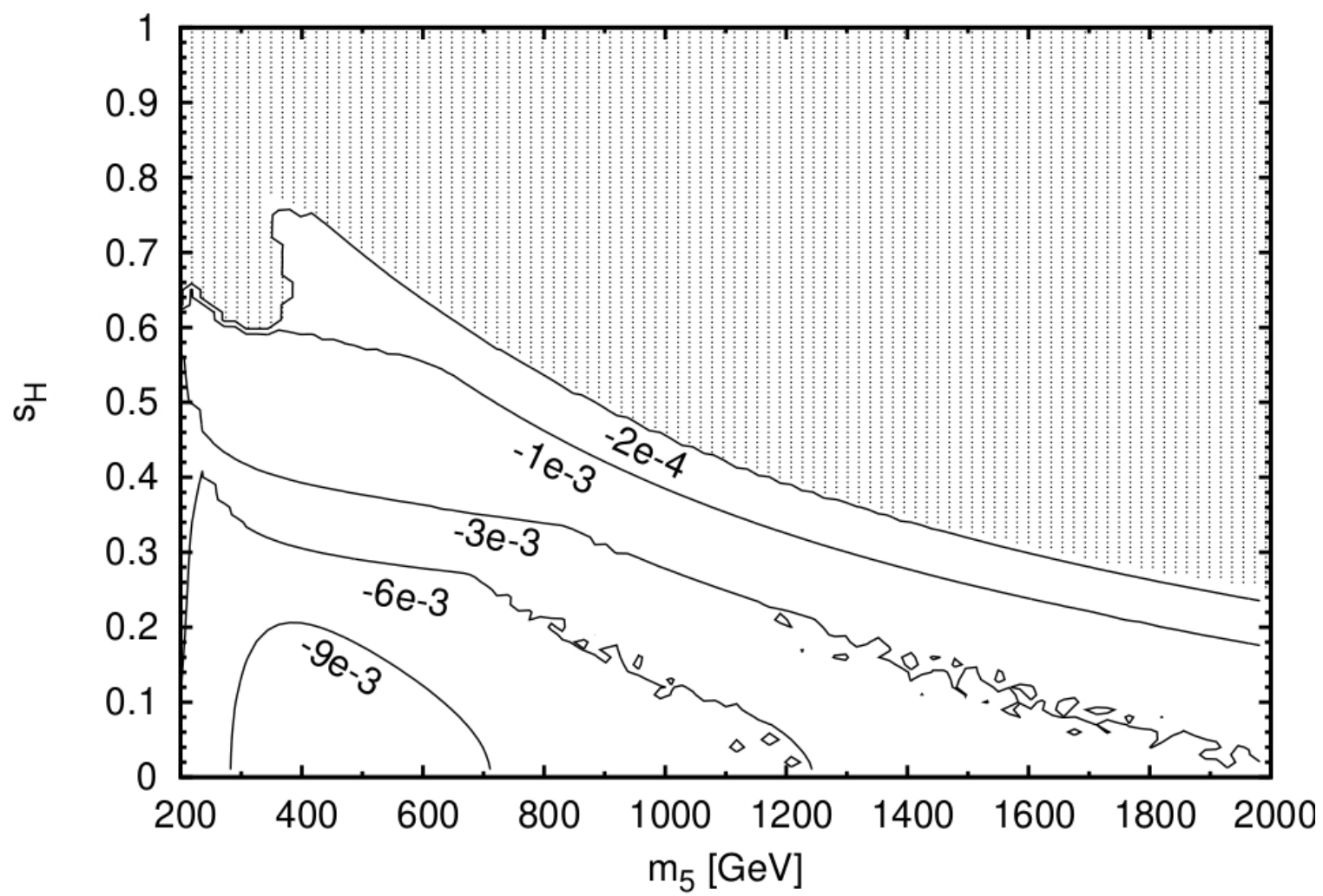

Figure 4.19: The fractional change in $\tilde{v}_{\chi}$ relative to the weak-scale custodialsymmetric input $v_{\chi}$, defined as $\frac{\tilde{v}_{\chi}}{v_{\chi}}-1$, in the H5plane benchmark taking the scale of the custodial-symmetric theory to be as large as possible subject to perturbative unitarity and the $\rho$ parameter constraint. The fractional change is always negative and its absolute value reaches a maximum of $1.0 \%$. 

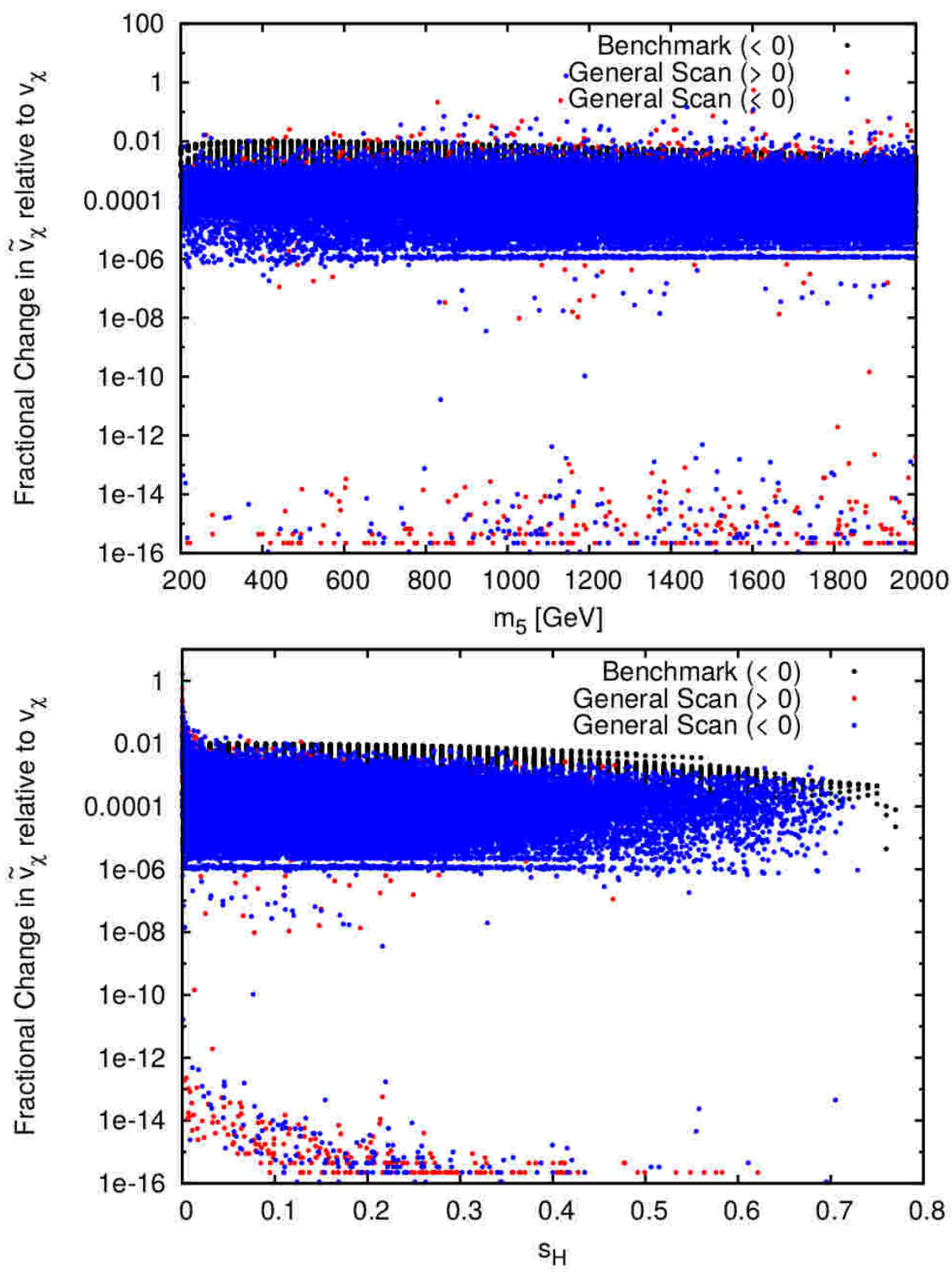

Figure 4.20: The fractional change in $\tilde{v}_{\chi}$ relative to the weak-scale custodialsymmetric input $v_{\chi}$, defined as $\frac{\tilde{v}_{\chi}}{v_{\chi}}-1$, as a function of $m_{5}$ (top) and $s_{H}$ (bottom), evaluated with the maximum allowed cutoff scale in a general parameter scan (blue and red points) and in the H5plane benchmark (black points). The fractional change can be positive (blue points) or negative (red points) in the general scan, but is always negative in the H5plane benchmark (black points). The fractional change reaches maxima and minima of 0.55 and -1.66 , respectively, at very low $s_{H}$, but for $s_{H}>0.1$ its absolute value reaches at most 0.0114 . 


\subsubsection{Low- $m_{5}$ region}

Finally in this subsection we present the results of a dedicated general scan of the low- $m_{5}$ region, focusing on $m_{5}<200 \mathrm{GeV}$. As usual, we take the cutoff as large as allowed by perturbative unitarity and the $\rho$ parameter constraint to maximize the amount of custodial symmetry violation.

In Fig. 4.21 we show the maximum allowed cutoff scale subject to perturbative unitarity of the quartic couplings in the custodial-symmetric theory and the $\rho$ parameter constraint, as a function of $m_{5}$ (top) and $s_{H}$ (bottom). Compared to the general scan for higher $m_{5}$, the maximum allowed cutoff tends to be lower, but large cutoff scales on the order of $100 \mathrm{TeV}$ are still somewhat common and the maximum cutoff scale found in our scan is of order $10^{10} \mathrm{GeV}$.

Subject to these constraints, in Fig. 4.22 we show the value of the $\rho$ parameter in the weak-scale theory, again as a function of $m_{5}$ (top) and $s_{H}$ (bottom). The scan populates the entirety of the allowed region; in particular, the small allowed region with $\rho<1$ is heavily populated. This is in contrast to the general scan at larger $m_{5}$, which strongly favours $\rho>1$.

We next consider custodial symmetry violation effects in couplings. In Fig. 4.23 we show the ratio $\lambda_{W Z}^{\tilde{h}}$ of the $125 \mathrm{GeV}$ Higgs boson's couplings to $W W$ and to $Z Z$. In the top panel we plot versus $m_{5}$ while the bottom panel zooms in to $\lambda_{W Z}^{\tilde{h}}$ between 0.9 and 1.1. The most dramatic feature is the resonant mixing when $\tilde{H}_{5}^{0}$ and $h$ become degenerate, for which deviations in $\lambda_{W Z}^{\tilde{h}}$ of tens of percent in either direction are possible. Such large mixing also substantially modifies the other couplings of $h$. Away from the resonant region, $\lambda_{W Z}^{\tilde{h}}$ can deviate from one by as much as $1-2 \%$, which is large enough to be probed at future $e^{+} e^{-}$colliders.

Of particular interest are decays of the would-be fermiophobic $H_{5}$ states to 

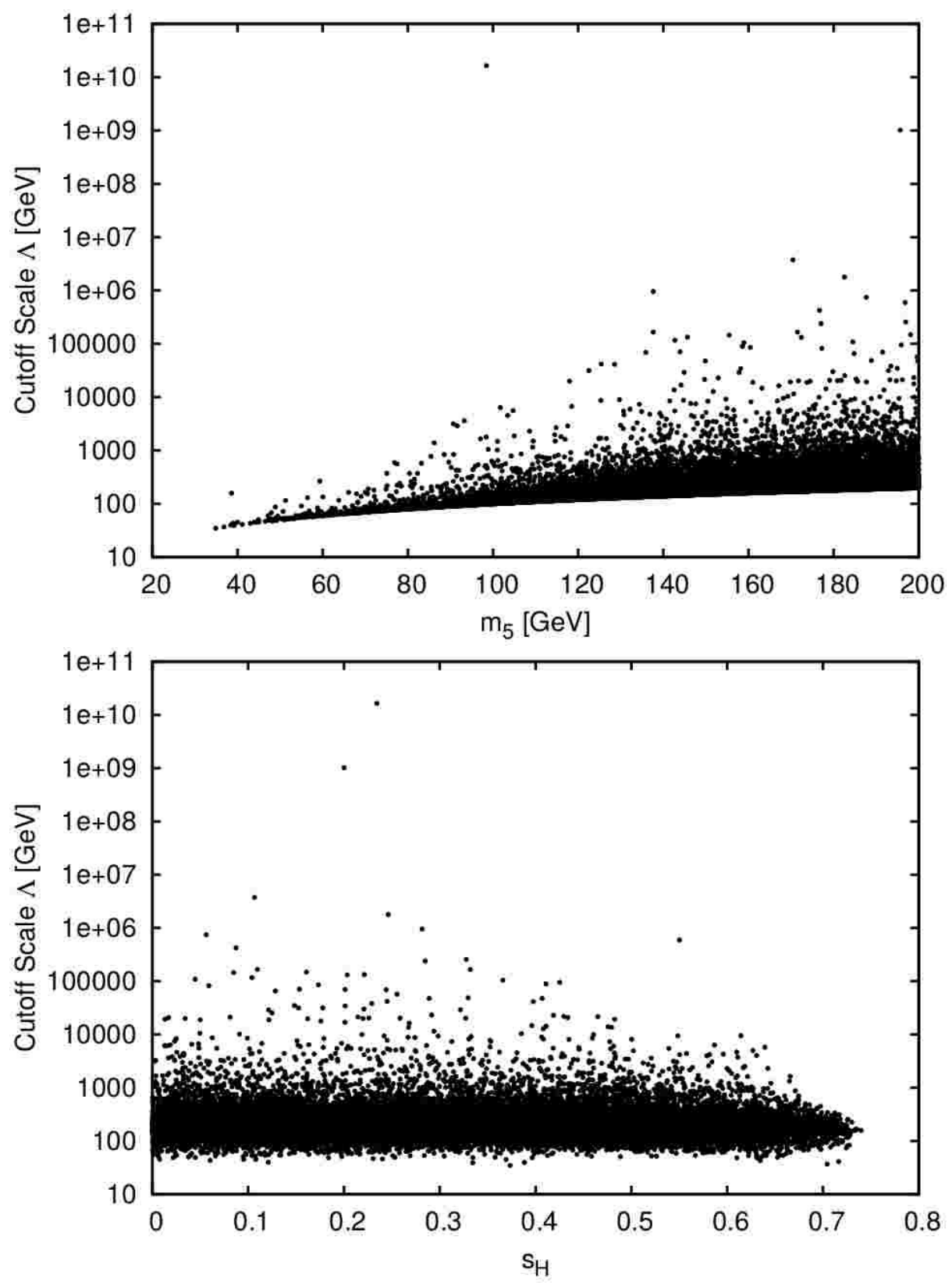

Figure 4.21: Maximum value of the custodial-symmetric cutoff scale subject to perturbative unitarity and the experimental constraint on the $\rho$ parameter in a general scan of the low- $m_{5}$ region, as a function of $m_{5}$ (top) and $s_{H}$ (bottom). The maximum cutoff scale ranges from $35 \mathrm{GeV}$ (for very low $m_{5}$ ) to $1.6 \times 10^{10} \mathrm{GeV}$. 

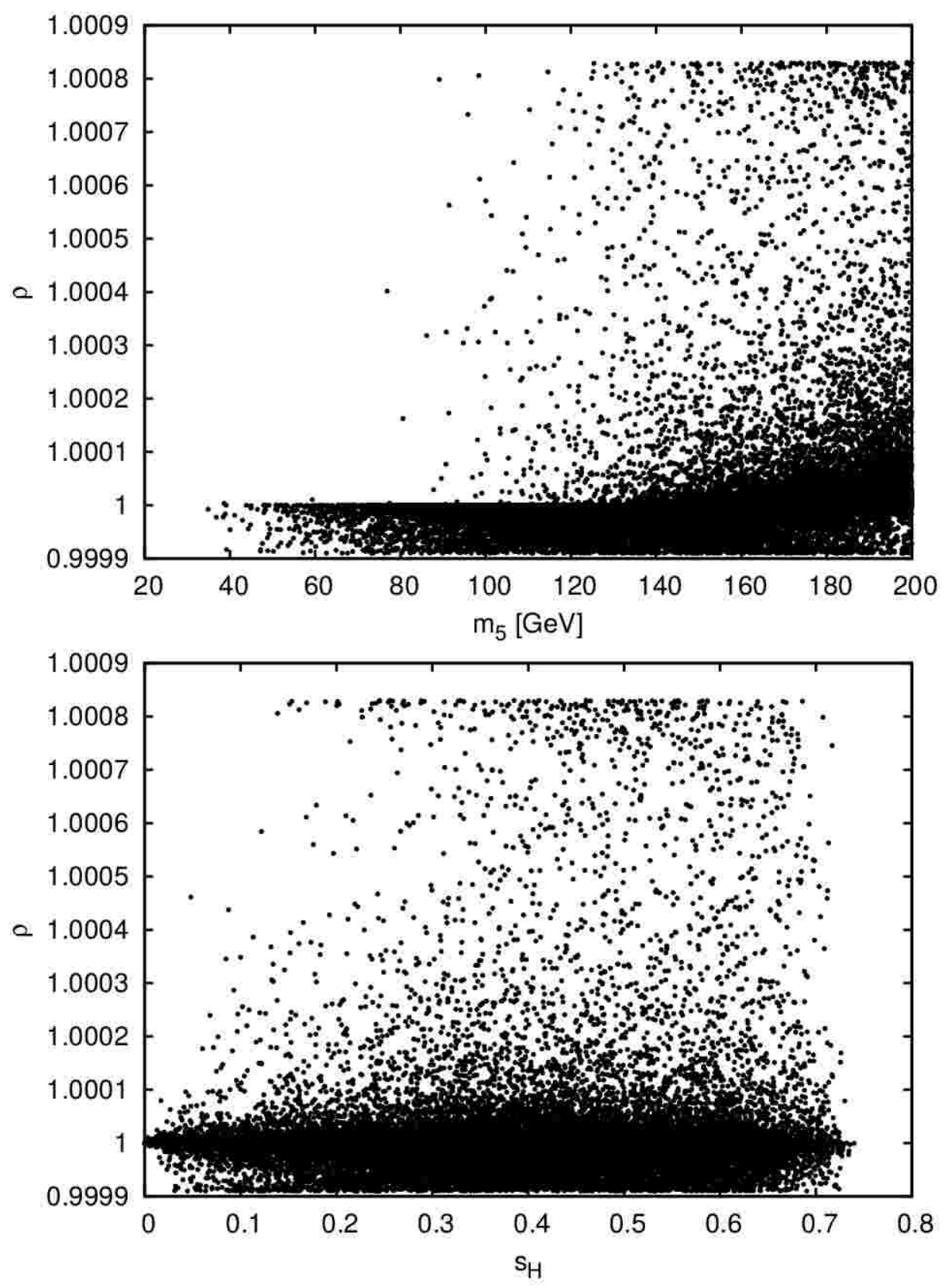

Figure 4.22: Value of the $\rho$ parameter in a general scan of the low- $m_{5}$ region as a function of $m_{5}$ (top) and $s_{H}$ (bottom), taking the scale of the custodial-symmetric theory to be as large as possible subject to perturbative unitarity and the $\rho$ parameter constraint. 

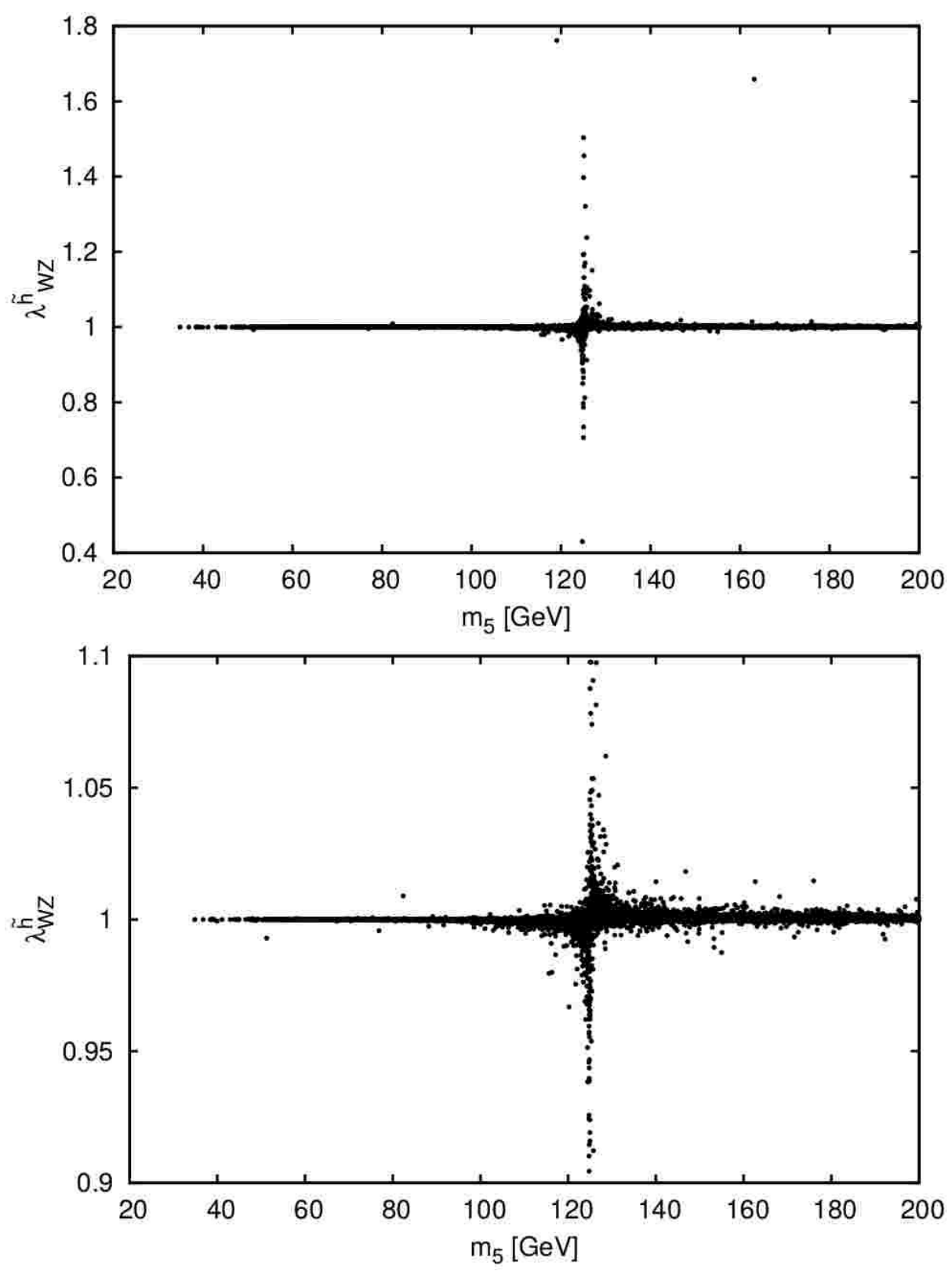

Figure 4.23: $\lambda_{W Z}^{\tilde{h}}$ in a general scan of the low- $m_{5}$ region as a function of $m_{5}$, taking the scale of the custodial-symmetric theory to be as large as possible subject to perturbative unitarity and the $\rho$ parameter constraint. The bottom panel is a zoom of the $y$-axis. In the resonant mixing region $m_{5} \simeq 125 \mathrm{GeV}$ we find values between 0.43 and 1.76 , while away from this region $\lambda_{W Z}^{\tilde{h}}$ can deviate from one by as much as $1-2 \%$. 
fermion pairs induced by custodial symmetry violation. We study this for $\tilde{H}_{5}^{+}$in Fig. 4.24. In the top panel we plot $\kappa_{f}^{\tilde{H}_{5}^{+}}$as a function of $m_{5}$. While the values are tiny for most scan points, they can reach values as large as about \pm 0.3 for $m_{5}$ close to $200 \mathrm{GeV}$. However, this does not change the overall pattern of $H_{5}^{+}$decays; as shown in the bottom panel of Fig. 4.24, the branching ratio into $W Z$ (red) continues to dominate, with the loop-induced decay into $W \gamma$ (green) becoming important for $m_{5}$ below the $W Z$ kinematic threshold. The branching ratio into fermions (black) remains small.

Decays of $H_{5}^{0}$ to fermion pairs are studied in Fig. 4.25. In the top panel we plot $\kappa_{f}^{\tilde{H}_{5}^{0}}$ as a function of $m_{5}$. The values are reasonably small except for $m_{5}$ around $125 \mathrm{GeV}$, where mixing between $H_{5}^{0}$ and $h$ becomes resonant. In the bottom panel we show the branching ratios of $\tilde{H}_{5}^{0}$. Decays to fermion pairs (black points) can become dominant only in the resonant-mixing region; away from $m_{5} \simeq 125 \mathrm{GeV}$ the branching ratio to fermion pairs generally remain below $10 \%$, including at very low $m_{5}$ values where the branching ratio into $\gamma \gamma$ (red) remains dominant. This is good news for the continued viability of the diphoton resonance search to constrain $\tilde{H}_{5}^{0}$ at low masses as proposed in Ref. [103].

Mass splittings among the members of the custodial fiveplet and triplet are shown in Fig. 4.26. As in the general scans for larger $m_{5}, \tilde{H}_{5}^{++}$tends to be the heaviest of the fiveplet states, followed by $\tilde{H}_{5}^{+}$, with $\tilde{H}_{5}^{0}$ the lightest. Likewise $\tilde{H}_{3}^{0}$ tends to be heavier than $\tilde{H}_{3}^{+}$, though this ordering can be reversed for a minority of the scan points. The approximate relation $m_{\tilde{H}_{5}^{++}}-m_{\tilde{H}_{5}^{0}} \simeq 4\left(m_{\tilde{H}_{5}^{+}}-m_{\tilde{H}_{5}^{0}}\right)$ holds true in the low- $m_{5}$ scan as well. The custodial-violating mass splittings are below $2 \mathrm{GeV}$ in most of the parameter space, and less than about $10 \mathrm{GeV}$ over the whole scan.

Finally in Fig. 4.27 we plot the fractional change in $\tilde{v}_{\chi}$ relative to the weak-scale 

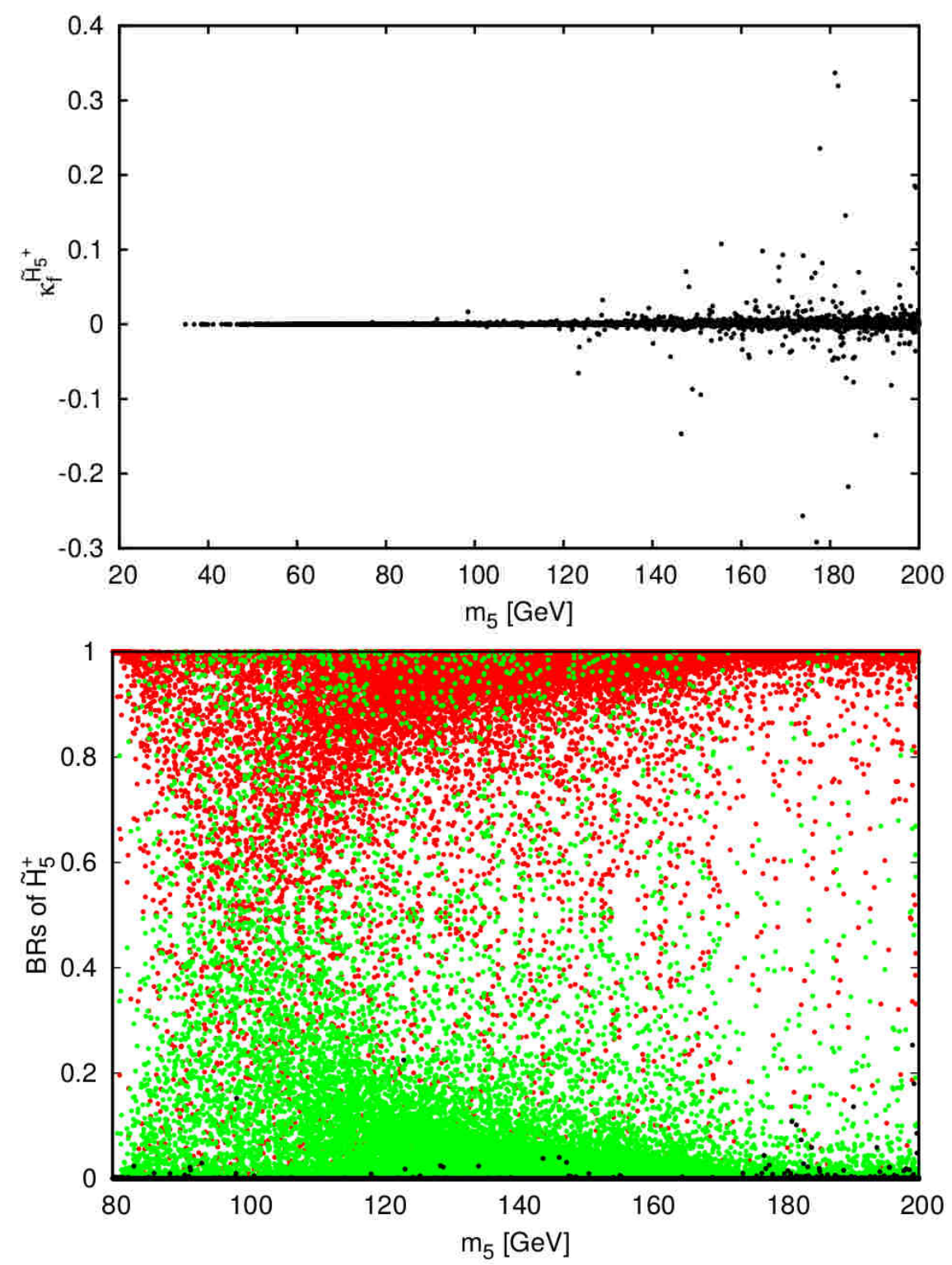

Figure 4.24: Custodial-symmetry-violation-induced couplings of $\tilde{H}_{5}^{+}$to fermions in a general scan of the low- $m_{5}$ region, taking the scale of the custodial-symmetric theory to be as large as possible subject to perturbative unitarity and the $\rho$ parameter constraint. Top: $\kappa_{f}^{\tilde{H}_{5}^{+}}$as a function of $m_{5}$. The minimum and maximum values are -0.29 and 0.34 respectively. Bottom: decay branching ratios of $\tilde{H}_{5}^{+}$to $W Z$ (red), $W \gamma$ (green), and $f \bar{f}$ (black) as a function of $m_{5}$. Decays to fermions are computed including only the dominant modes: $t b$ above the $t b$ threshold and $c s$ and $c d$ below. The calculation of $H_{5}^{+} \rightarrow W \gamma$ assumes an on-shell final-state $W$, so we plot the branching ratios only between 80 and $200 \mathrm{GeV}$. 

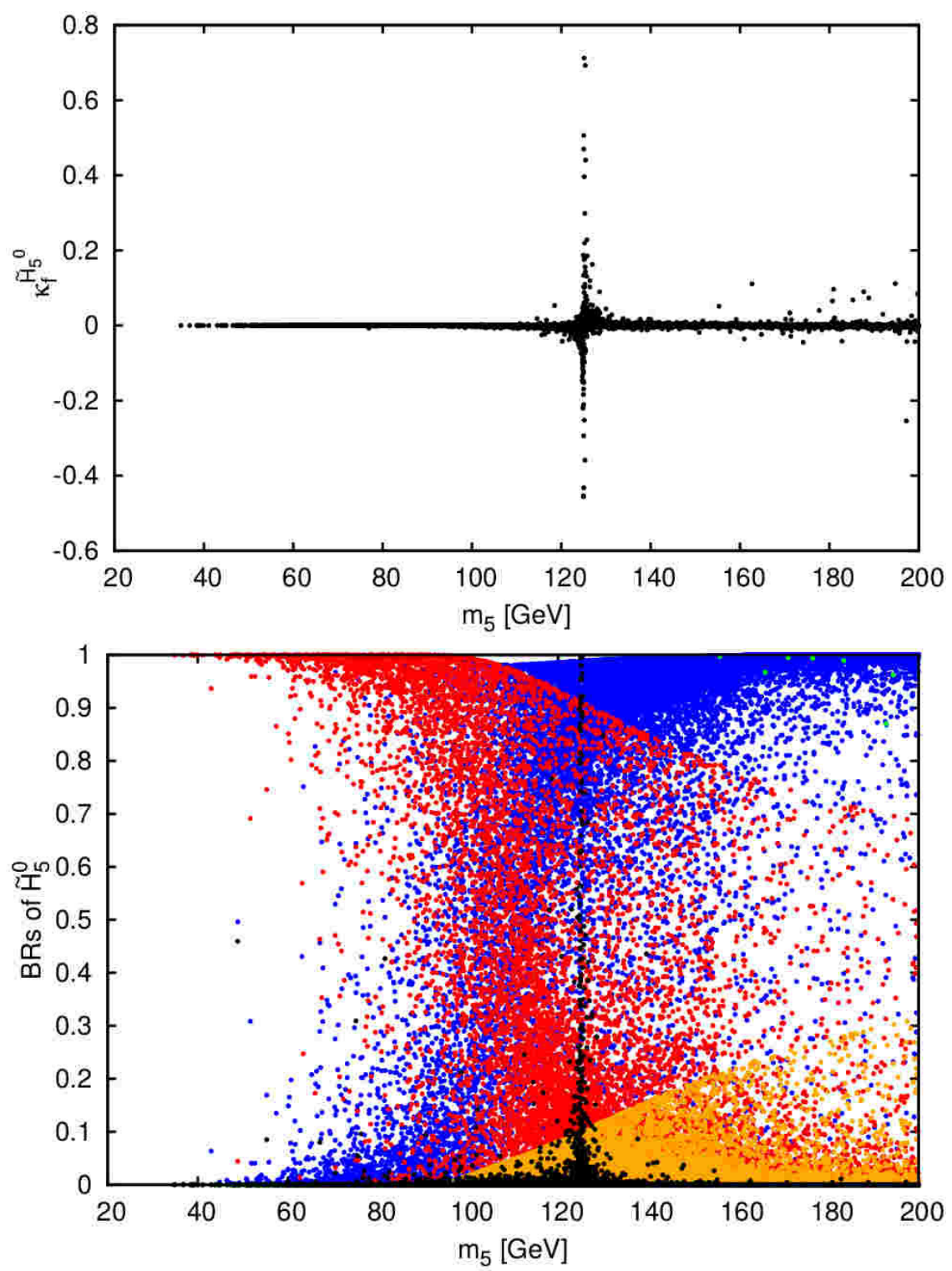

Figure 4.25: Custodial-symmetry-violation-induced couplings of $\tilde{H}_{5}^{0}$ to fermions in a general scan of the low- $m_{5}$ region, taking the scale of the custodial-symmetric theory to be as large as possible subject to perturbative unitarity and the $\rho$ parameter constraint. Top: $\kappa_{f}^{\tilde{H}_{5}^{0}}$ as a function of $m_{5}$. The minimum and maximum values are -0.46 and 0.71 respectively. Bottom: decay branching ratios of $\tilde{H}_{5}^{0}$ to $W W / Z Z$ (blue), $\gamma \gamma$ (red), $f \bar{f}$ (black), $Z \gamma$ (orange), and custodial-violating decays to pairs of other scalars (green - a few points in the upper right of the plot). 

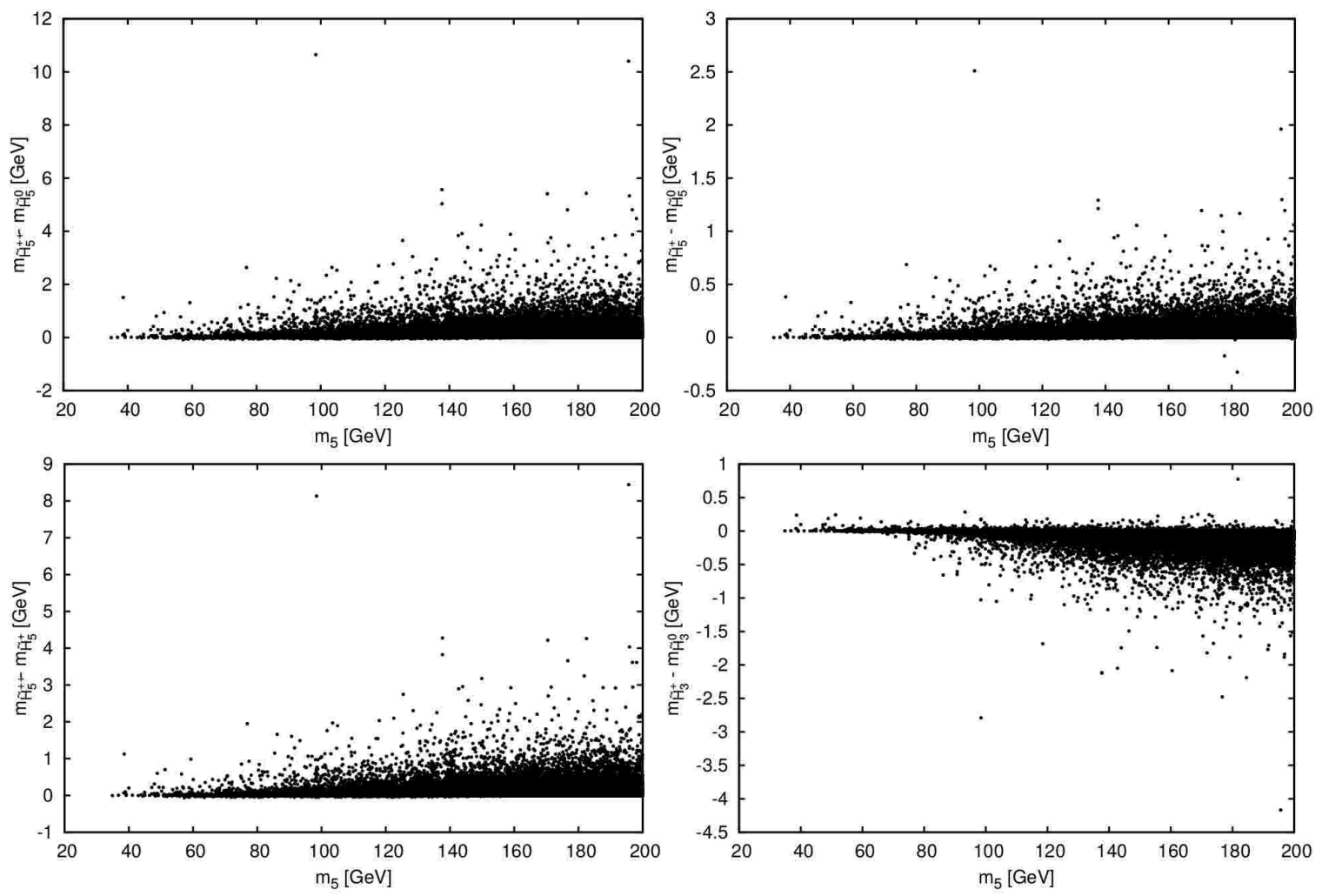

Figure 4.26: Mass splittings among the members of the custodial fiveplet and triplet in a general scan of the low- $m_{5}$ region, taking the scale of the custodial-symmetric theory to be as large as possible subject to perturbative unitarity and the $\rho$ parameter constraint. For the fiveplet we show $m_{\tilde{H}_{5}^{++}}-m_{\tilde{H}_{5}^{0}}$ (top left, ranging between $-0.08 \mathrm{GeV}$ and $10.6 \mathrm{GeV}$ ), $m_{\tilde{H}_{5}^{+}}-m_{\tilde{H}_{5}^{0}}$ (top right, ranging between $-0.33 \mathrm{GeV}$ and $2.51 \mathrm{GeV}$ ), and $m_{\tilde{H}_{5}^{++}}-m_{\tilde{H}_{5}^{+}}$(bottom left, ranging between $-0.06 \mathrm{GeV}$ and $8.44 \mathrm{GeV}$ ), and for the triplet we show $m_{\tilde{H}_{3}^{+}}-m_{\tilde{H}_{3}^{0}}$ (bottom right, ranging between $-4.17 \mathrm{GeV}$ and $0.78 \mathrm{GeV})$. 
custodial-symmetric input $v_{\chi}$, defined as $\frac{\tilde{v}_{\chi}}{v_{\chi}}-1$. This deviation can be positive (black points) or negative (red points), though negative deviations tend to occur only for $m_{5}$ above $100 \mathrm{GeV}$. The absolute value of the deviation is again small, below the percent level unless $s_{H}$ is very small.

\subsection{Summary}

In this chapter we studied the effects of custodial symmetry violation in the GeorgiMachacek model. We considered the scenario in which the exactly custodial-symmetric GM model emerges at some high scale $\Lambda$ as an effective low energy theory of an unspecified ultraviolet completion, and then ran the model down to the weak scale, through which running the hypercharge interactions give rise to custodial symmetry violation at one loop. The amount and pattern of custodial symmetry violation at the weak scale, as manifested through the couplings and masses of the physical scalars, is uniquely determined by the parameters of the high-scale custodial-symmetric theory and the value of the scale $\Lambda$ and hence can be meaningfully constrained by the measured value of the electroweak $\rho$ parameter.

To implement this program we used the most general gauge invariant scalar potential for the theory, from which we computed the minimization conditions for the vevs, and the expressions for the physical scalar mass eigenstates. These allowed us to calculate the custodial symmetry violating couplings of the physical $\tilde{H}_{5}^{0}$ and $\tilde{H}_{5}^{+}$states to fermions, as well as the parameter $\lambda_{W Z}^{\tilde{h}} \equiv \kappa_{W}^{\tilde{h}} / \kappa_{Z}^{\tilde{h}}$ for the $125 \mathrm{GeV}$ Higgs boson. My collaborator Terry Pilkington rederived the renormalization group equations for the parameters of the most general scalar potential including $\mathrm{CP}$ violation, and confirmed the results of Ref. [24] in the CP-conserving limit. In our numerical implementation of the RGE running we self-consistently adjusted the custodial-symmetric inputs to 

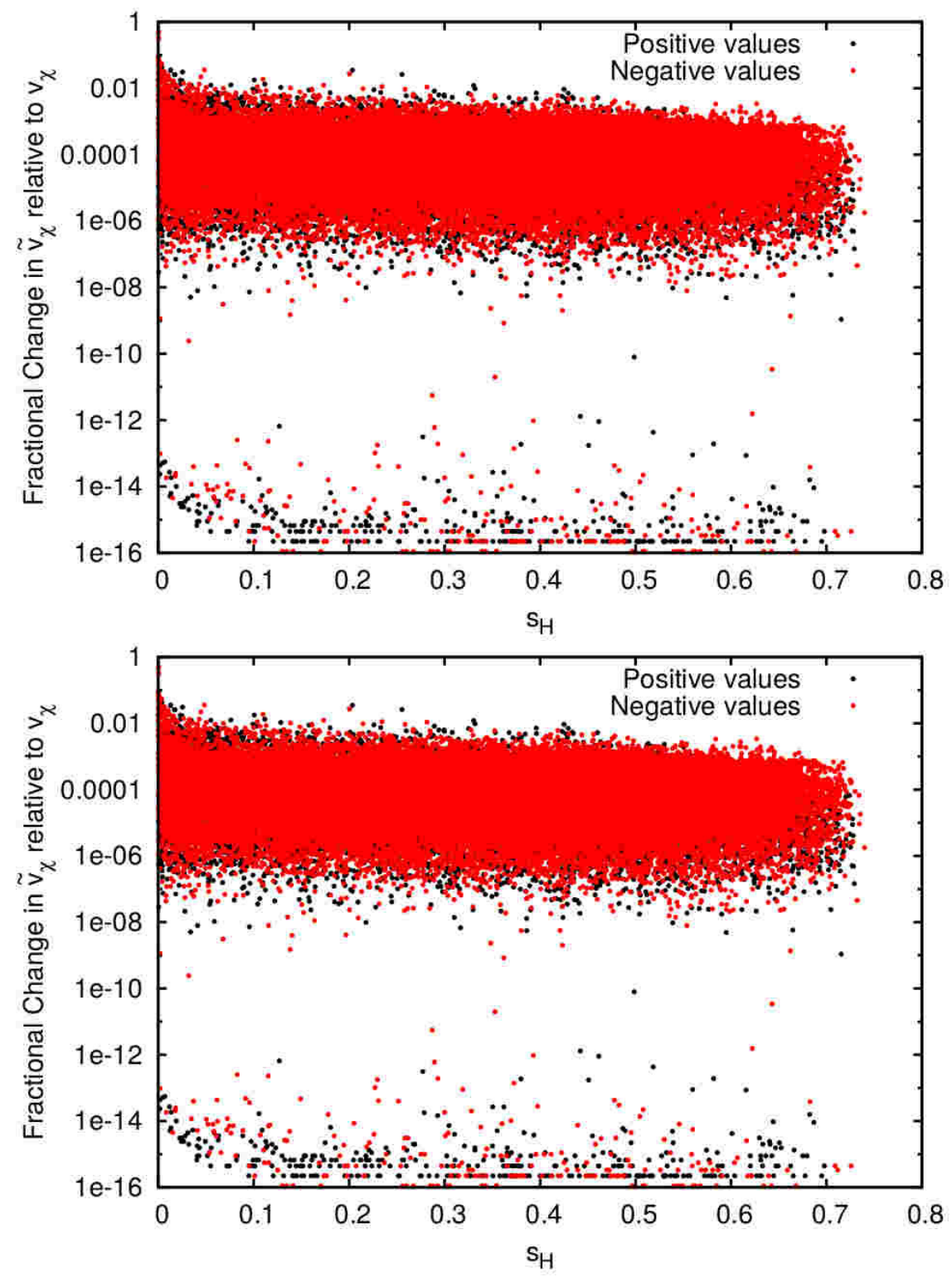

Figure 4.27: Fractional deviation of $\tilde{v}_{\chi}$ relative to the weak-scale custodialsymmetric input $v_{\chi}$, defined as $\frac{\tilde{v}_{\chi}}{v_{\chi}}-1$, as a function of $m_{5}$ (top) and $s_{H}$ (bottom) in a general scan of the low- $m_{5}$ region, taking the scale of the custodial-symmetric theory to be as large as possible subject to perturbative unitarity and the $\rho$ parameter constraint. Positive deviations are shown in black and negative in red so that both can be plotted on a log scale. The fractional deviation ranges between -1.66 and 0.55 , with these large deviations appearing mainly at very small $s_{H}$. 
obtain the correct values of the physical $125 \mathrm{GeV}$ Higgs boson mass, top quark mass, and Fermi constant $G_{F}$ in the weak-scale custodial-violating theory.

We presented numerical results in the H5plane benchmark (which helped make evident patterns like the relationship between the $\tilde{H}_{5}^{++}-\tilde{H}_{5}^{+}$and the $\tilde{H}_{5}^{+}-\tilde{H}_{5}^{0}$ mass splittings) as well as a general scan over the full parameter space. We showed that the results in the H5plane benchmark are broadly typical of the full parameter scan, though more extreme values can be obtained in small regions of parameter space in the general scan, particularly when the custodial-symmetric mass spectrum is such that the mixing among scalars in different custodial representations becomes resonant. We also performed a dedicated general scan for low $m_{5}<200 \mathrm{GeV}$ which is not captured in the H5plane benchmark.

In each case, we determined the maximum allowed scale of the custodialsymmetric theory imposing perturbative unitarity of two-to-two scalar scattering amplitudes and the experimental constraint on the $\rho$ parameter. This allowed us to quantify the maximum possible deviation of $\lambda_{W Z}^{\tilde{h}}$ from its SM value, as well as the branching ratios of the otherwise-fermiophobic $\tilde{H}_{5}^{0}$ and $\tilde{H}_{5}^{ \pm}$scalars into fermions and the mass splittings within the custodial triplet and fiveplet. We found that the scale of the custodial-symmetric theory could be as high as tens to hundreds of $\mathrm{TeV}$, with an upper bound of $290 \mathrm{TeV}$ in the H5plane benchmark. We showed that $\lambda_{W Z}^{\tilde{h}}$ can deviate from its SM value by at most two per mille when $m_{5}>200 \mathrm{GeV}$, though larger deviations at the percent level are possible in the low- $m_{5}$ region even away from the resonant mixing region $m_{5} \simeq m_{h}$. We also showed that the mass splittings within the custodial triplet and the custodial fiveplet are below $10 \mathrm{GeV}$ over almost the entire parameter space, reaching larger values only for large scalar masses. Both of these custodial-violating effects are too small to be probed at the LHC, but may be 
detectable at a future $e^{+} e^{-}$collider. Finally we showed that the fermionic branching ratios of $\tilde{H}_{5}^{0}$ and $\tilde{H}_{5}^{+}$remain below the $10 \%$ level, even for $\tilde{H}_{5}$ masses below the $W W$ and $W Z$ thresholds where they can compete directly with the loop-induced $\gamma \gamma$ and $W \gamma$ decay modes (with the exception of a narrow region of resonant mixing between $H_{5}^{0}$ and $h$ at $125 \mathrm{GeV}$ ). This preserves the usefulness of the $\gamma \gamma$ decay mode to put strong constraints on $\tilde{H}_{5}^{0}$ at low masses.

From these results, we can draw two important conclusions about the GM model. The generically small custodial-violating effects allows us to conclude that the use of the custodial-symmetric GM model as a benchmark model for LHC searches is justified. Furthermore, the large upper bound on the scale of the UV completion suggests that virtual effects from particles at the UV completion scale will be highly suppressed and their contribution to effective operators measured at the LHC will be too small to detect. This means that not only is the GM model a useful benchmark at the LHC but it is also a valid effective theory at the weak scale. 


\section{Chapter 5}

\section{Conclusion}

In this thesis we examined the effects of custodial symmetry violation in the GeorgiMachacek model. The GM model is a useful benchmark model for 'exotic' Higgs searches at the LHC. Custodial symmetry is one of the key features of the tree-level GM model but it is violated at one-loop. This violation raises several significant questions about the model. What is a natural scale for the GM model's ultraviolet completion and does the highly constraining measurement of the electro-weak $\rho$ parameter force the UV scale to be close to the weak scale? And can custodial symmetry violation allow the model to escape the current constraints from collider searches? To answer these questions, we treated the GM model as a low energy effective theory of some unspecified ultraviolet completion with an accidental custodial symmetry at some high scale $\Lambda$. At this high scale the model possessed exact custodial symmetry. Using the one-loop renormalization group equations we then ran the model down to the weak scale with the contribution to the running from hypercharge interactions introducing custodial symmetry violation. Fixing the parameters of the high-scale

custodial-symmetric theory and the value of the scale $\Lambda$ gives a unique pattern of custodial symmetry violation at the weak scale. This gives an unambiguous predic- 
tion for the value of the $\rho$ parameter as a function of $\Lambda$, which allowed us to place an upper bound on $\Lambda$. The custodial symmetry violation also manifests in the couplings and masses of the physical scalars which allowed us to quantify the deviation from the custodial symmetric tree-level theory.

To implement the above framework we used the most general gauge invariant scalar potential for the theory, which we used to compute the minimization conditions for the vevs, and the expressions for the physical scalar mass eigenstates. From these we calculated the custodial symmetry violating couplings of the physical $\tilde{H}_{5}^{0}$ and $\tilde{H}_{5}^{+}$ states to fermions, as well as the parameter $\lambda_{W Z}^{\tilde{h}} \equiv \kappa_{W}^{\tilde{h}} / \kappa_{Z}^{\tilde{h}}$ for the $125 \mathrm{GeV}$ Higgs boson. To facilitate our comparison with the custodial symmetric tree-level theory, we choose to use the parameters of the custodial symmetric weak scale tree-level theory as our inputs rather than to directly use our inputs at the high scale $\Lambda$. In our numerical implementation of the RGE running we self-consistently adjusted the custodial-symmetric inputs to obtain the correct values of the physical $125 \mathrm{GeV}$ Higgs boson mass, top quark mass, and Fermi constant $G_{F}$ in the weak-scale custodialviolating theory.

Our numerical results were presented as contours in the H5plane benchmark (where patterns in the custodial symmetry violation of various observables was more readily apparent) and as a general scan over the full parameter space. We showed that while the results in the H5plane benchmark are generally typical of the full parameter scan, more extreme values can be obtained in small regions of parameter space in the general scan. This is particularly true for parameter points which allow for resonant mixing among scalars in different custodial representations as this can greatly enhance custodial violating effects. We also performed a dedicated general scan for low $m_{5}<200 \mathrm{GeV}$ which lies outside the H5plane benchmark. 
In each case, we determined the maximal value of $\Lambda$ by imposing perturbative unitarity of two-to-two scalar scattering amplitudes of the high scale custodial symmetric theory and the experimental constraint on the $\rho$ parameter. These maximal values correspond to the maximum amount of custodial symmetry violation for each parameter point. This allowed us to calculate the maximum possible deviation of $\lambda_{W Z}^{\tilde{h}}$ from its SM value, the branching ratios of the otherwise-fermiophobic $\tilde{H}_{5}^{0}$ and $\tilde{H}_{5}^{ \pm}$ scalars into fermions, and the mass splittings within the custodial triplet and fiveplet. We found that the scale of the custodial-symmetric theory could be as high as tens to hundreds of $\mathrm{TeV}$, with an upper bound of $290 \mathrm{TeV}$ in the H5plane benchmark. We demonstrated that these custodial-violating effects are generally too small to be probed at the LHC showing that $\lambda_{W Z}^{\tilde{h}}$ can deviate from its SM value by at most two per mille when $m_{5}>200 \mathrm{GeV}$ (this is not necessarily the case in the low- $m_{5}$ region which allows for deviations at the percent level even away from the resonant mixing region $\left.m_{5} \simeq m_{h}\right)$ and that the mass splittings within the custodial triplet and the custodial fiveplet are below $10 \mathrm{GeV}$ over almost the entire parameter space, reaching larger values only for large scalar masses. These tiny deviations and splittings may be detectable at a future $e^{+} e^{-}$collider. Finally we showed that the fermionic branching ratios of $\tilde{H}_{5}^{0}$ and $\tilde{H}_{5}^{+}$are small throughout the parameter space. This is true even for $\tilde{H}_{5}$ masses below the $W W$ and $W Z$ thresholds where they can compete directly with the loop-induced $\gamma \gamma$ and $W \gamma$ decay modes as the fermionic decays generally remain below the $10 \%$ level (this is not true in the narrow region of resonant mixing between $H_{5}^{0}$ and $h$ at $125 \mathrm{GeV}$ where these decays dominate). This preserves the usefulness of the $\gamma \gamma$ decay mode to put strong constraints on $\tilde{H}_{5}^{0}$ at low masses.

From these results, we can answer the questions raised by the spectre of custodial symmetry violation and draw two important conclusions about the GM model. 
As virtual effects coming from the additional particles of the UV completion are suppressed by inverse powers of the UV scale, our upper bound suggests that their contribution to effective operators measured at the LHC will be undetectable. Thus we can conclude that the GM model is a valid effective theory at the weak scale. Additionally, the generically small custodial-violating effects justifies the use of the tree-level custodial-symmetric GM model as a benchmark model for LHC searches.

In this thesis, we also introduced a new benchmark for the GM model designed to cover the eponymous low- $m_{5}$ region neglected by the H5plane benchmark. We defined the low- $m_{5}$ benchmark, for $m_{5} \in(50,550) \mathrm{GeV}$. The benchmark, designed to facilitate searches for $H_{5}^{++}, H_{5}^{+}$, and $H_{5}^{0}$ with $m_{5}<200 \mathrm{GeV}$, highlights an unexcluded region of parameter space with light additional Higgs bosons, providing additional motivation to design searches which explore this region of parameter space. In our design, we ensure $B R\left(H_{5}^{++} \rightarrow W^{+} W^{+}\right)=1$ by choosing $m_{3}>m_{5}$ throughout the benchmark plane, while simultaneously avoiding large modifications to $h \rightarrow \gamma \gamma$ from loops of light charged scalars. Using a pre-release version of the public code GMCALC 1.5.0 for our numerical work, we examined the region's existing experimental constraints, which limit $s_{H}$ to be strictly below 0.63 in the benchmark, and studied the benchmark's phenomenology.

In our study of the low- $m_{5}$ benchmark's phenomenology, we showed that the SM-like Higgs boson's tree-level couplings to fermion and vector boson pairs are universally enhanced, with enhancements as large as $19 \%$ and $8 \%$, respectively, possible. We also showed that even with these modifications, the SM-like Higgs boson always satisfies the Higgs signal strength constraints. We also examined a benchmark-specific enhancement to the $H_{5}$ pair production cross sections by the process $p p \rightarrow H \rightarrow H_{5} H_{5}$ and showed that it is always at least an order of magnitude smaller than the compet- 
ing Drell-Yan production of $H_{5}$ pairs (we anticipate that a dedicated LHC search for the Drell-Yan production could exclude the entirety of the $m_{5}<200 \mathrm{GeV}$ region). Finally, we showed that diphoton resonance search constraints require $B R\left(H_{5}^{0} \rightarrow \gamma \gamma\right)$ to be less than about $10 \%$ and that this in turn forces $B R\left(H_{5}^{+} \rightarrow W \gamma\right)$ to be less than about $2 \%$. We also pointed out that the narrow width approximation is justified throughout the benchmark as the width-to-mass ratios of the $H_{5}$ states are below $1 \%$. 


\section{Appendix A}

\section{Renormalization group equations}

\section{for Lagrangian parameters}

In order to run the parameters down from a custodial-symmetric high scale to the weak scale, we need the RGEs for the parameters of the most general gauge-invariant potential as given in Eq. (4.13). RGEs can be calculated with the public codes PyR@TE [46], a Python code that generates two-loop RGEs for non-supersymmetric models, and SARAH [47], a Mathematica package which can generate two-loop RGEs for supersymmetric and non-supersymmetric models. PyR@TE requires the user to supply their own GM model card while SARAH provides a GM model card in an alternate parameterization of the scalar potential. We determine the RGES using the formalism presented in Ref. [45]. The resulting equations are then (with $t \equiv \log \mu$, where $\mu$ is the energy scale),

$$
\begin{array}{r}
16 \pi^{2} \frac{d\left(\tilde{\mu}_{2}^{2}\right)}{d t}=\frac{3}{2} \tilde{M}_{1}^{2}+3\left|\tilde{M}_{1}^{\prime}\right|^{2}+\tilde{\mu}_{2}^{2}\left(6 y_{b}^{2}+6 y_{t}^{2}+2 y_{\tau}^{2}-\frac{9}{10} g_{1}^{2}-\frac{9}{2} g_{2}^{2}+12 \tilde{\lambda}_{1}\right) \\
+6 \tilde{\mu}_{3}^{2} \tilde{\lambda}_{6}+6 \tilde{\mu}_{3}^{\prime 2} \tilde{\lambda}_{5}
\end{array}
$$




$$
\begin{aligned}
& 16 \pi^{2} \frac{d\left(\tilde{\mu}_{3}^{\prime 2}\right)}{d t}=\left|\tilde{M}_{1}^{\prime}\right|^{2}+144 \tilde{M}_{2}^{2}+\tilde{\mu}_{3}^{\prime 2}\left(8 \tilde{\lambda}_{2}+16 \tilde{\lambda}_{7}-\frac{18}{5} g_{1}^{2}-12 g_{2}^{2}\right)+4 \tilde{\mu}_{2}^{2} \tilde{\lambda}_{5} \\
& +2 \tilde{\mu}_{3}^{2}\left(\tilde{\lambda}_{9}+3 \tilde{\lambda}_{10}\right), \\
& 16 \pi^{2} \frac{d\left(\tilde{\mu}_{3}^{2}\right)}{d t}=\tilde{M}_{1}^{2}+144 \tilde{M}_{2}^{2}+4 \tilde{\mu}_{3}^{2}\left(10 \tilde{\lambda}_{8}-3 g_{2}^{2}\right)+8 \tilde{\mu}_{2}^{2} \tilde{\lambda}_{6}+4 \tilde{\mu}_{3}^{\prime 2}\left(\tilde{\lambda}_{9}+3 \tilde{\lambda}_{10}\right) \\
& 16 \pi^{2} \frac{d \tilde{\lambda}_{1}}{d t}=-6 y_{b}^{4}-6 y_{t}^{4}-2 y_{\tau}^{4}+\tilde{\lambda}_{1}\left(12 y_{b}^{2}+12 y_{t}^{2}+4 y_{\tau}^{2}-\frac{9}{5} g_{1}^{2}-9 g_{2}^{2}+24 \tilde{\lambda}_{1}\right) \\
& +\frac{27}{200} g_{1}^{4}+\frac{9}{8} g_{2}^{4}+\frac{9}{20} g_{1}^{2} g_{2}^{2}+\frac{1}{2} \tilde{\lambda}_{3}^{2}+2\left|\tilde{\lambda}_{4}\right|^{2}+3 \tilde{\lambda}_{5}^{2}+6 \tilde{\lambda}_{6}^{2}, \\
& 16 \pi^{2} \frac{d \tilde{\lambda}_{2}}{d t}=3 g_{2}^{4}-\frac{36}{5} g_{1}^{2} g_{2}^{2}+12 \tilde{\lambda}_{2}\left(\tilde{\lambda}_{2}+2 \tilde{\lambda}_{7}-\frac{3}{5} g_{1}^{2}-2 g_{2}^{2}\right)-\frac{1}{2} \tilde{\lambda}_{3}^{2}+\tilde{\lambda}_{9}^{2}, \\
& 16 \pi^{2} \frac{d \tilde{\lambda}_{3}}{d t}=\tilde{\lambda}_{3}\left(6 y_{b}^{2}+6 y_{t}^{2}+2 y_{\tau}^{2}+4 \tilde{\lambda}_{1}-8 \tilde{\lambda}_{2}+8 \tilde{\lambda}_{5}+4 \tilde{\lambda}_{7}-\frac{9}{2} g_{1}^{2}-\frac{33}{2} g_{2}^{2}\right) \\
& +\frac{36}{5} g_{2}^{2} g_{1}^{2}+4\left|\tilde{\lambda}_{4}\right|^{2} \\
& 16 \pi^{2} \frac{d \tilde{\lambda}_{4}}{d t}=\tilde{\lambda}_{4}\left(6 y_{b}^{2}+6 y_{t}^{2}+2 y_{\tau}^{2}-\frac{27}{10} g_{1}^{2}-\frac{33}{2} g_{2}^{2}+4 \tilde{\lambda}_{1}+2 \tilde{\lambda}_{3}+4 \tilde{\lambda}_{5}+8 \tilde{\lambda}_{6}\right. \\
& \left.-2 \tilde{\lambda}_{9}+4 \tilde{\lambda}_{10}\right) \\
& 16 \pi^{2} \frac{d \tilde{\lambda}_{5}}{d t}=\tilde{\lambda}_{5}\left(6 y_{b}^{2}+6 y_{t}^{2}+2 y_{\tau}^{2}+4 \tilde{\lambda}_{5}+12 \tilde{\lambda}_{1}+8 \tilde{\lambda}_{2}+16 \tilde{\lambda}_{7}-\frac{9}{2} g_{1}^{2}-\frac{33}{2} g_{2}^{2}\right) \\
& +\frac{27}{25} g_{1}^{4}+6 g_{2}^{4}+2 \tilde{\lambda}_{3}^{2}+4\left|\tilde{\lambda}_{4}\right|^{2}+4 \tilde{\lambda}_{6} \tilde{\lambda}_{9}+12 \tilde{\lambda}_{6} \tilde{\lambda}_{10} \\
& 16 \pi^{2} \frac{d \tilde{\lambda}_{6}}{d t}=\tilde{\lambda}_{6}\left(6 y_{b}^{2}+6 y_{t}^{2}+2 y_{\tau}^{2}+8 \tilde{\lambda}_{6}+12 \tilde{\lambda}_{1}+40 \tilde{\lambda}_{8}-\frac{9}{10} g_{1}^{2}-\frac{33}{2} g_{2}^{2}\right) \\
& +3 g_{2}^{4}+4\left|\tilde{\lambda}_{4}\right|^{2}+2 \tilde{\lambda}_{5} \tilde{\lambda}_{9}+6 \tilde{\lambda}_{5} \tilde{\lambda}_{10} \\
& 16 \pi^{2} \frac{d \tilde{\lambda}_{7}}{d t}=\frac{54}{25} g_{1}^{4}+9 g_{2}^{4}+\frac{36}{5} g_{2}^{2} g_{1}^{2}+\left(-\frac{36}{5} g_{1}^{2}-24 g_{2}^{2}+16 \tilde{\lambda}_{2}+28 \tilde{\lambda}_{7}\right) \\
& +16 \tilde{\lambda}_{2}^{2}+\frac{1}{2} \tilde{\lambda}_{3}^{2}+2 \tilde{\lambda}_{5}^{2}+\tilde{\lambda}_{9}^{2}+2 \tilde{\lambda}_{10}\left(3 \tilde{\lambda}_{10}+2 \tilde{\lambda}_{9}\right), \\
& 16 \pi^{2} \frac{d \tilde{\lambda}_{8}}{d t}=3 g_{2}^{4}+8 \tilde{\lambda}_{8}\left(-3 g_{2}^{2}+11 \tilde{\lambda}_{8}\right)+2 \tilde{\lambda}_{6}^{2}+\tilde{\lambda}_{9}\left(\tilde{\lambda}_{9}+2 \tilde{\lambda}_{10}\right)+3 \tilde{\lambda}_{10}^{2},
\end{aligned}
$$




$$
\begin{array}{r}
16 \pi^{2} \frac{d \tilde{\lambda}_{9}}{d t}=6 g_{2}^{4}+2 \tilde{\lambda}_{9}\left(-12 g_{2}^{2}-\frac{9}{5} g_{1}^{2}+5 \tilde{\lambda}_{9}+4 \tilde{\lambda}_{2}+2 \tilde{\lambda}_{7}+8 \tilde{\lambda}_{8}+8 \tilde{\lambda}_{10}\right) \\
-2\left|\tilde{\lambda}_{4}\right|^{2} \\
16 \pi^{2} \frac{d \tilde{\lambda}_{10}}{d t}=6 g_{2}^{4}+2 \tilde{\lambda}_{10}\left(-\frac{9}{5} g_{1}^{2}-12 g_{2}^{2}+4 \tilde{\lambda}_{2}+8 \tilde{\lambda}_{7}+20 \tilde{\lambda}_{8}+4 \tilde{\lambda}_{10}\right) \\
+2\left|\tilde{\lambda}_{4}\right|^{2}+2 \tilde{\lambda}_{9}^{2}+4 \tilde{\lambda}_{5} \tilde{\lambda}_{6}+4 \tilde{\lambda}_{9}\left(\tilde{\lambda}_{7}+2 \tilde{\lambda}_{8}\right) \\
16 \pi^{2} \frac{d \tilde{M}_{1}^{\prime}}{d t}=\tilde{M}_{1}^{\prime}\left(6 y_{b}^{2}+6 y_{t}^{2}+2 y_{\tau}^{2}-\frac{27}{10} g_{1}^{2}-\frac{21}{2} g_{2}^{2}+4 \tilde{\lambda}_{1}+4 \tilde{\lambda}_{3}+4 \tilde{\lambda}_{5}\right) \\
+4 \sqrt{2} \tilde{\lambda}_{4}^{*}\left(\tilde{M}_{1}+6 \tilde{M}_{2}\right) \\
16 \pi^{2} \frac{d \tilde{M}_{1}}{d t}=\tilde{M}_{1}\left(6 y_{b}^{2}+6 y_{t}^{2}+2 y_{\tau}^{2}-\frac{9}{10} g_{1}^{2}-\frac{21}{2} g_{2}^{2}+4 \tilde{\lambda}_{1}+8 \tilde{\lambda}_{6}\right)+24 \tilde{M}_{2} \tilde{\lambda}_{3} \\
+8 \sqrt{2} \operatorname{Re}\left[\tilde{M}_{1}^{\prime} \tilde{\lambda}_{4}\right] \\
16 \pi^{2} \frac{d \tilde{M}_{2}}{d t}=\tilde{M}_{2}\left(-\frac{18}{5} g_{1}^{2}-18 g_{2}^{2}-8 \tilde{\lambda}_{2}+4 \tilde{\lambda}_{7}-4 \tilde{\lambda}_{9}+8 \tilde{\lambda}_{10}\right)+\frac{1}{6} \tilde{M}_{1} \tilde{\lambda}_{3} \\
+\frac{1}{3} \sqrt{2} \operatorname{Re}\left[\tilde{M}_{1}^{\prime} \tilde{\lambda}_{4}\right]
\end{array}
$$

where $g_{1}$ and $g_{2}$ are gauge couplings (see below) and $y_{b}, y_{t}$, and $y_{\tau}$ are Yukawa couplings, normalized according to $y_{f}=\sqrt{2} m_{f} / \tilde{v}_{\phi}$. These RGEs agree with those of Ref. [24] (for real $\tilde{\lambda}_{4}$ and $\tilde{M}_{1}^{\prime}$ ) after translating the notation for the Lagrangian parameters as follows:

$$
\begin{aligned}
\sigma_{1} & =-\frac{\tilde{\lambda}_{3}}{2}+\tilde{\lambda}_{5}, \\
\sigma_{2} & =\tilde{\lambda}_{3}, \\
\sigma_{3} & =\tilde{\lambda}_{6}, \\
\sigma_{4} & =\tilde{\lambda}_{4},
\end{aligned}
$$




$$
\begin{aligned}
\lambda & =\tilde{\lambda}_{1}, \\
\rho_{1} & =2 \tilde{\lambda}_{2}+\tilde{\lambda}_{7}, \\
\rho_{2} & =-2 \tilde{\lambda}_{2}, \\
\rho_{3} & =2 \tilde{\lambda}_{8}, \\
\rho_{4} & =\tilde{\lambda}_{10}, \\
\rho_{5} & =\tilde{\lambda}_{9}, \\
\mu_{1} & =\frac{\tilde{M}_{1}}{\sqrt{2}}, \\
\mu_{2} & =\frac{\tilde{M}_{1}^{\prime}}{2}, \\
\mu_{3} & =-6 \sqrt{2} \tilde{M}_{2}, \\
m_{\phi}^{2} & =\tilde{\mu}_{2}^{2}, \\
m_{\chi}^{2} & =\tilde{\mu}_{3}^{\prime 2}, \\
m_{\xi}^{2} & =\frac{\tilde{\mu}_{3}^{2}}{2} .
\end{aligned}
$$

A few possible symmetries are apparent in these RGEs. Setting $\tilde{M}_{1}^{\prime}=\tilde{M}_{1}=$ $\tilde{M}_{2}=0$, the potential becomes invariant under $(\chi, \xi) \rightarrow(-\chi,-\xi)$ and therefore these three parameters are not regenerated by the running. Setting instead $\tilde{\lambda}_{4}=\tilde{M}_{1}^{\prime}=0$, the potential becomes invariant under $\chi \rightarrow-\chi$ and therefore these two parameters are not regenerated by the running. Setting $\tilde{\lambda}_{4}=\tilde{M}_{1}=\tilde{M}_{2}=0$, the potential becomes invariant under $\xi \rightarrow-\xi$ and therefore these three parameters are not regenerated by the running. Finally, if all the Lagrangian parameters are taken to be real at some scale, as will be the case when the most general potential is matched onto the intrinsically CP-conserving custodial-symmetric Georgi-Machacek model, they remain real at all scales. 
Throughout we use the GUT normalization $g^{\prime}=\sqrt{\frac{3}{5}} g_{1}, g=g_{2}$, and $g_{s}=g_{3}$. The renormalization group equations for the electroweak gauge couplings, including all the particle content of the GM model in the spectrum, are [109],

$$
\begin{aligned}
16 \pi^{2} \frac{d g_{1}}{d t} & =\frac{47}{10} g_{1}^{3} \quad \text { or equivalently } \quad 16 \pi^{2} \frac{d g^{\prime}}{d t}=\frac{47}{6} g^{\prime 3} \\
16 \pi^{2} \frac{d g_{2}}{d t} & =-\frac{13}{6} g_{2}^{3},
\end{aligned}
$$

and that for the strong gauge coupling is the same as in the SM (including the top quark contribution),

$$
16 \pi^{2} \frac{d g_{3}}{d t}=-7 g_{3}^{3}
$$

The RGEs for the Yukawa couplings are identical to those of the SM [110],

$$
\begin{gathered}
16 \pi^{2} \frac{d y_{t}}{d t}=\left(-\frac{17}{20} g_{1}^{2}-\frac{9}{4} g_{2}^{2}-8 g_{3}^{2}+\frac{3}{2} y_{b}^{2}+\frac{9}{2} y_{t}^{2}+y_{\tau}^{2}\right) y_{t} \\
16 \pi^{2} \frac{d y_{b}}{d t}=\left(-\frac{1}{4} g_{1}^{2}-\frac{9}{4} g_{2}^{2}-8 g_{3}^{2}+\frac{9}{2} y_{b}^{2}+\frac{3}{2} y_{t}^{2}+y_{\tau}^{2}\right) y_{b} \\
16 \pi^{2} \frac{d y_{\tau}}{d t}=\left(-\frac{9}{4} g_{1}^{2}-\frac{9}{4} g_{2}^{2}+3 y_{b}^{2}+3 y_{t}^{2}+\frac{5}{2} y_{\tau}^{2}\right) y_{\tau} .
\end{gathered}
$$

In our numerical work we will ignore $y_{b}$ and $y_{\tau}$.

As a consistency check, we can turn off the custodial-violating parts of the RGEs by setting $g_{1}=0$ and substituting the relations given in Eq. (4.15). We then find a self-consistent set of RGEs for the custodial-preserving Lagrangian parameters:

$$
\begin{aligned}
16 \pi^{2} \frac{d\left(\mu_{2}^{2}\right)}{d t} & =\frac{9}{2} M_{1}^{2}+\mu_{2}^{2}\left(6 y_{b}^{2}+6 y_{t}^{2}+2 y_{\tau}^{2}-\frac{9}{2} g_{2}^{2}+48 \lambda_{1}\right)+36 \mu_{3}^{2} \lambda_{2}, \\
16 \pi^{2} \frac{d\left(\mu_{3}^{2}\right)}{d t} & =M_{1}^{2}+144 M_{2}^{2}+16 \mu_{2}^{2} \lambda_{2}+\mu_{3}^{2}\left(-12 g_{2}^{2}+56 \lambda_{3}+88 \lambda_{4}\right),
\end{aligned}
$$




$$
\begin{gathered}
16 \pi^{2} \frac{d \lambda_{1}}{d t}=-\frac{3}{2} y_{b}^{4}-\frac{3}{2} y_{t}^{4}-\frac{1}{2} y_{\tau}^{4}+\lambda_{1}\left(12 y_{b}^{2}+12 y_{t}^{2}+4 y_{\tau}^{2}-9 g_{2}^{2}+96 \lambda_{1}\right) \\
+\frac{9}{32} g_{2}^{4}+18 \lambda_{2}^{2}+\frac{3}{2} \lambda_{5}^{2} \\
16 \pi^{2} \frac{d \lambda_{2}}{d t}=\lambda_{2}\left(6 y_{b}^{2}+6 y_{t}^{2}+2 y_{\tau}^{2}-\frac{33}{2} g_{2}^{2}+48 \lambda_{1}+16 \lambda_{2}+56 \lambda_{3}+88 \lambda_{4}\right) \\
+\frac{3}{2} g_{2}^{4}+4 \lambda_{5}^{2}, \\
16 \pi^{2} \frac{d \lambda_{3}}{d t}=\frac{3}{2} g_{2}^{4}+\lambda_{3}\left(-24 g_{2}^{2}+80 \lambda_{3}+96 \lambda_{4}\right)-\lambda_{5}^{2} \\
16 \pi^{2} \frac{d \lambda_{4}}{d t}=\frac{3}{2} g_{2}^{4}+\lambda_{4}\left(-24 g_{2}^{2}+136 \lambda_{4}+112 \lambda_{3}\right)+8 \lambda_{2}^{2}+24 \lambda_{3}^{2}+\lambda_{5}^{2}, \\
16 \pi^{2} \frac{d \lambda_{5}}{d t}=\lambda_{5}\left(6 y_{b}^{2}+6 y_{t}^{2}+2 y_{\tau}^{2}-\frac{33}{2} g_{2}^{2}+16 \lambda_{1}+32 \lambda_{2}-8 \lambda_{3}+16 \lambda_{4}\right. \\
16 \pi^{2} \frac{d M_{1}}{d t}=M_{1}\left(6 y_{b}^{2}+6 y_{t}^{2}+2 y_{\tau}^{2}-\frac{21}{2} g_{2}^{2}+16 \lambda_{1}+16 \lambda_{2}-16 \lambda_{5}\right)-48 M_{2} \lambda_{5}, \\
16 \pi^{2} \frac{d M_{2}}{d t}=-M_{1} \lambda_{5}+M_{2}\left(-18 g_{2}^{2}-24 \lambda_{3}+48 \lambda_{4}\right) \\
(\mathrm{A})
\end{gathered}
$$




\section{Appendix B}

\section{Scalar couplings of the custodial}

\section{violating states}

In this appendix we give the 3 -scalar couplings for the custodial symmetric eigenstates used in the calculation of $H_{5}$ decays after custodial symmetry violation.

The modified couplings for decays to scalars allowed by custodial symmetry:

$$
\begin{aligned}
g_{H_{5}^{0} H_{3}^{+} H_{3}^{-}} & =\tilde{\lambda}_{3}\left(\frac{s_{H} c_{H} \tilde{v}_{\phi}}{2 \sqrt{3}}+\frac{s_{H}^{2} \tilde{v}_{\chi}}{\sqrt{6}}\right)+\tilde{\lambda}_{4} \frac{1}{\sqrt{6}} s_{H} c_{H} \tilde{v}_{\phi}-\tilde{\lambda}_{5} \frac{2 \sqrt{2}}{\sqrt{3}} s_{H}^{2} \tilde{v}_{\chi} \\
& +\tilde{\lambda}_{6} \frac{4 \sqrt{2}}{\sqrt{3}} s_{H}^{2} \tilde{v}_{\xi}-\tilde{\lambda}_{7} \frac{\sqrt{2}}{\sqrt{3}} c_{H}^{2} \tilde{v}_{\chi}+\frac{4 \sqrt{2}}{\sqrt{3}} \tilde{\lambda}_{8} c_{H}^{2} \tilde{v}_{\xi} \\
& +\tilde{\lambda}_{9} \frac{\sqrt{2}}{\sqrt{3}}\left(c_{H}^{2} \tilde{v}_{\xi}-\frac{c_{H}^{2} \tilde{v}_{\chi}}{2}+\frac{c_{H}^{2} \tilde{v}_{\xi}}{2}-c_{H}^{2} \tilde{v}_{\chi}\right) \\
& +\tilde{\lambda}_{10} \frac{\sqrt{2}}{\sqrt{3}} c_{H}^{2}\left(\tilde{v}_{\xi}-\tilde{v}_{\chi}\right)+\tilde{M}_{1} \frac{\sqrt{2}}{\sqrt{3}} s_{H}^{2}-\sqrt{6} c_{H}^{2} \tilde{M}_{2},
\end{aligned}
$$




$$
\begin{aligned}
& g_{H_{5}^{+} \tilde{H}_{3}^{0} \tilde{H}_{3}^{0}}=-\tilde{\lambda}_{3} \frac{8 \tilde{v}_{\chi}^{3}}{\sqrt{6} A^{2}}+\frac{\tilde{\lambda}_{4}}{\sqrt{3} A^{2}}\left(8 \tilde{v}_{\xi} \tilde{v}_{\chi}^{2}-16 \tilde{v}_{\chi}^{3}-8 \tilde{v}_{\chi} \tilde{v}_{\phi}^{2}\right)-\tilde{\lambda}_{5} \frac{16 \tilde{v}_{\chi}^{3}}{\sqrt{6} A^{2}} \\
& +\tilde{\lambda}_{6} \frac{16 \sqrt{2} \tilde{v}_{\chi}^{2} \tilde{v}_{\xi}}{\sqrt{3} A^{2}}-\tilde{\lambda}_{7} \frac{4 \tilde{v}_{\chi} \tilde{v}_{\phi}^{2}}{\sqrt{6} A^{2}}+\tilde{\lambda}_{10} \frac{2 \sqrt{2} \tilde{v}_{\phi}^{2} \tilde{v}_{\xi}}{\sqrt{3} A^{2}}-\tilde{M}_{1}^{\prime} \frac{8 \tilde{v}_{\chi}^{2}}{\sqrt{6} A^{2}} \\
& -\tilde{M}_{1} \frac{8 \tilde{v}_{\chi}^{2}}{\sqrt{6} A^{2}}-\tilde{M}_{2} \frac{6 \sqrt{2} \tilde{v}_{\phi}^{2}}{\sqrt{3} A^{2}} \\
& g_{H_{5}^{+} H_{3}^{+} \tilde{H}_{3}^{0}}=-\frac{i}{A}\left(\tilde{\lambda}_{3}\left(s_{H} \tilde{v}_{\chi}^{2}+\frac{s_{H} \tilde{v}_{\phi}^{2}}{4}\right)+\tilde{\lambda}_{4}\left(\sqrt{2} s_{H} \tilde{v}_{\chi}^{2}+2 c_{H} \tilde{v}_{\phi} \tilde{v}_{\chi}+\frac{s_{H} \tilde{v}_{\phi}^{2}}{2 \sqrt{2}}\right)\right. \\
& \left.-\tilde{\lambda}_{9} \frac{c_{H} \tilde{v}_{\phi} \tilde{v}_{\xi}}{\sqrt{2}}+\tilde{M}_{1}^{\prime} \tilde{v}_{\chi} s_{H}-\tilde{M}_{1} s_{H} \tilde{v}_{\chi}-\tilde{M}_{2} \frac{6 c_{H} \tilde{v}_{\phi}}{\sqrt{2}}\right), \\
& g_{H_{5}^{++} H_{3}^{-} H_{3}^{-}}=-2\left(-\tilde{\lambda}_{2} c_{H}^{2} \tilde{v}_{\chi}-\tilde{\lambda}_{3} \frac{s_{H} c_{H} \tilde{v}_{\phi}}{2 \sqrt{2}}+\tilde{\lambda}_{4}\left(\frac{s_{H}^{2} \tilde{v}_{\xi}}{\sqrt{2}}-\frac{s_{H} c_{H} \tilde{v}_{\phi}}{2}\right)-\tilde{M}_{1}^{\prime} \frac{s_{H}^{2}}{2}\right. \\
& \left.-3 \tilde{M}_{2} c_{H}^{2}\right) \\
& g_{H_{5}^{++} H_{5}^{-} H_{5}^{-}}=-2\left(-\tilde{\lambda}_{2} \tilde{v}_{\chi}+3 \tilde{M}_{2}\right)
\end{aligned}
$$

where $A^{2}=\tilde{v}_{\phi}^{2}+8 \tilde{v}_{\chi}^{2}$.

The modified couplings for loop decays mediated by a $H_{5}$ loop:

$$
\begin{aligned}
g_{H_{5}^{0} H_{5}^{+} H_{5}^{-}} & =-\tilde{\lambda}_{7} \frac{\tilde{v}_{\chi}}{\sqrt{6}}+\tilde{\lambda}_{8} \frac{4 \sqrt{2} \tilde{v}_{\xi}}{\sqrt{3}}+\tilde{\lambda}_{9}\left(\frac{\sqrt{2} \tilde{v}_{\xi}}{\sqrt{3}}-\frac{\tilde{v}_{\chi}}{6}-\frac{\tilde{v}_{\xi}}{6}+\frac{\sqrt{2} \tilde{v}_{\chi}}{\sqrt{3}}\right) \\
& +\tilde{\lambda}_{10}\left(\frac{\sqrt{2} \tilde{v}_{\xi}}{\sqrt{3}}-\frac{2 \tilde{v}_{\chi}}{6}\right)-\sqrt{6} \tilde{M}_{2} \\
g_{H_{5}^{0} H_{5}^{++} H_{5}^{--}} & =-\tilde{\lambda}_{2} \frac{8 \tilde{v}_{\chi}}{\sqrt{6}}-\tilde{\lambda}_{7} \frac{4 \tilde{v}_{\chi}}{\sqrt{6}}+\tilde{\lambda}_{10} \frac{2 \sqrt{2} \tilde{v}_{\xi}}{\sqrt{3}}-\frac{6 \sqrt{2}}{\sqrt{3}} \tilde{M}_{2} .
\end{aligned}
$$


The couplings for decays to scalars that violate custodial symmetry:

$$
\begin{aligned}
& g_{H_{5}^{0} h_{\tilde{\alpha}} h_{\tilde{\alpha}}}=\frac{\tilde{\lambda}_{3}}{\sqrt{2}}\left(-\frac{\tilde{v}_{\chi} c_{\tilde{\alpha}}^{2}}{2 \sqrt{3}}+\frac{\tilde{v}_{\phi} c_{\tilde{\alpha}} s_{\tilde{\alpha}}}{3}\right)+\tilde{\lambda}_{4}\left(-\frac{\tilde{v}_{\phi} c_{\tilde{\alpha}} s_{\tilde{\alpha}}}{3}+c_{\tilde{\alpha}}^{2}\left(\frac{\tilde{v}_{\chi}}{\sqrt{3}}-\frac{\tilde{v}_{\xi}}{2 \sqrt{3}}\right)\right) \\
& +\tilde{\lambda}_{5}\left(-\frac{\tilde{v}_{\chi} c_{\tilde{\tilde{\alpha}}}^{2}}{\sqrt{6}}+\frac{\sqrt{2} c_{\tilde{\alpha}} s_{\tilde{\alpha}} \tilde{v}_{\phi}}{3}\right)+\tilde{\lambda}_{6}\left(\frac{\sqrt{2} \tilde{v}_{\xi} c_{\tilde{\alpha}}^{2}}{\sqrt{3}}-\frac{2 \sqrt{2} \tilde{v}_{\phi} c_{\tilde{\alpha}} s_{\tilde{\alpha}}}{3}\right) \\
& -\tilde{\lambda}_{7} \frac{2 \sqrt{2} \tilde{v}_{\chi} s_{\tilde{\alpha}}^{2}}{\sqrt{3}}+\tilde{\lambda}_{8} \frac{4 \sqrt{2} \tilde{v}_{\xi} s_{\tilde{\alpha}}^{2}}{\sqrt{3}}+\tilde{\lambda}_{10} \frac{\sqrt{2} \tilde{v}_{\chi} s_{\tilde{\alpha}}^{2}}{\sqrt{3}}+\tilde{M}_{1}^{\prime} \frac{c_{\tilde{\alpha}}^{2}}{2 \sqrt{6}}-\tilde{M}_{1} \frac{c_{\tilde{\alpha}}^{2}}{2 \sqrt{6}}, \\
& g_{H_{5}^{0} H_{\tilde{\alpha}} H_{\tilde{\alpha}}}=-\frac{\tilde{\lambda}_{3}}{\sqrt{2}}\left(\frac{\tilde{v}_{\chi} s_{\tilde{\alpha}}^{2}}{2 \sqrt{3}}+\frac{\tilde{v}_{\phi} c_{\tilde{\alpha}} s_{\tilde{\alpha}}}{3}\right)+\tilde{\lambda}_{4}\left(\frac{\tilde{v}_{\phi} c_{\tilde{\alpha}} s_{\tilde{\alpha}}}{3}+s_{\tilde{\alpha}}^{2}\left(\frac{\tilde{v}_{\chi}}{\sqrt{3}}-\frac{\tilde{v}_{\xi}}{2 \sqrt{3}}\right)\right) \\
& +\tilde{\lambda}_{5}\left(-\frac{\tilde{v}_{\chi} s_{\tilde{\alpha}}^{2}}{\sqrt{6}}-\frac{\sqrt{2} c_{\tilde{\alpha}} s_{\tilde{\alpha}} \tilde{v}_{\phi}}{3}\right)+\tilde{\lambda}_{6}\left(\frac{\sqrt{2} \tilde{v}_{\xi} s_{\tilde{\alpha}}^{2}}{\sqrt{3}}+\frac{2 \sqrt{2} \tilde{v}_{\phi} c_{\tilde{\alpha}} s_{\tilde{\alpha}}}{3}\right) \\
& -\tilde{\lambda}_{7} \frac{2 \sqrt{2} \tilde{v}_{\chi} c_{\tilde{\alpha}}^{2}}{\sqrt{3}}+\tilde{\lambda}_{8} \frac{4 \sqrt{2} \tilde{v}_{\xi} c_{\tilde{\alpha}}^{2}}{\sqrt{3}}+\tilde{\lambda}_{10} \frac{\sqrt{2} \tilde{v}_{\chi} c_{\tilde{\alpha}}^{2}}{\sqrt{3}}+\tilde{M}_{1}^{\prime} \frac{s_{\tilde{\tilde{\alpha}}}^{2}}{2 \sqrt{6}}-\tilde{M}_{1} \frac{s_{\tilde{\alpha}}^{2}}{2 \sqrt{6}}, \\
& g_{H_{5}^{0} h_{\tilde{\alpha}} H_{\tilde{\alpha}}}=-\frac{\tilde{\lambda}_{3}}{\sqrt{2}}\left(\frac{\tilde{v}_{\chi} s_{\tilde{\alpha}} c_{\tilde{\alpha}}}{\sqrt{3}}+\frac{\tilde{v}_{\phi} c_{2 \tilde{\alpha}}}{3}\right)+\tilde{\lambda}_{4}\left(\frac{\tilde{v}_{\phi} c_{2 \tilde{\alpha}}}{3}+2 s_{\tilde{\alpha}} c_{\tilde{\alpha}}\left(\frac{\tilde{v}_{\chi}}{\sqrt{3}}-\frac{\tilde{v}_{\xi}}{2 \sqrt{3}}\right)\right) \\
& +\tilde{\lambda}_{5}\left(-2 \frac{\tilde{v}_{\chi} s_{\tilde{\alpha}} c_{\tilde{\alpha}}}{\sqrt{6}}-\frac{\sqrt{2} c_{2 \tilde{\alpha}} \tilde{v}_{\phi}}{3}\right)+\tilde{\lambda}_{6}\left(\frac{2 \sqrt{2} \tilde{v}_{\xi} s_{\tilde{\alpha}} c_{\tilde{\alpha}}}{\sqrt{3}}+\frac{2 \sqrt{2} \tilde{v}_{\phi} c_{2 \tilde{\alpha}}}{3}\right) \\
& -\tilde{\lambda}_{7} \frac{4 \sqrt{2} \tilde{v}_{\chi} c_{\tilde{\alpha}} s_{\tilde{\alpha}}}{\sqrt{3}}-\tilde{\lambda}_{8} \frac{8 \sqrt{2} \tilde{v}_{\xi} c_{\tilde{\alpha}} s_{\tilde{\alpha}}}{\sqrt{3}}-\tilde{\lambda}_{10} \frac{2 \sqrt{2} \tilde{v}_{\chi} c_{\tilde{\alpha}} s_{\tilde{\alpha}}}{\sqrt{3}}+\tilde{M}_{1}^{\prime} \frac{s_{\tilde{\alpha}} c_{\tilde{\alpha}}}{\sqrt{6}} \\
& -\tilde{M}_{1} \frac{s_{\tilde{\alpha}} c_{\tilde{\alpha}}}{\sqrt{6}} \text {. }
\end{aligned}
$$




\section{References}

[1] A. Ismail, B. Keeshan, H. E. Logan and Y. Wu, "Benchmark for LHC searches for low-mass custodial fiveplet scalars in the Georgi-Machacek model," Phys. Rev. D 103, no.9, 095010 (2021) [arXiv:2003.05536 [hep-ph]].

[2] B. Keeshan, H. E. Logan and T. Pilkington, "Custodial symmetry violation in the Georgi-Machacek model," Phys. Rev. D 102, no.1, 015001 (2020) [arXiv:1807.11511 [hep-ph]].

[3] D. A. Ross and M. J. G. Veltman, "Neutral Currents in Neutrino Experiments," Nucl. Phys. B 95, 135 (1975); M. J. G. Veltman, "Limit on Mass Differences in the Weinberg Model," Nucl. Phys. B 123, 89 (1977).

[4] P.A. Zyla et al. (Particle Data Group), "Review of Particle Physics,", Prog. Theor. Exp. Phys. 2020, $083 \mathrm{C} 01$ (2020).

[5] J. Hisano and K. Tsumura, "Higgs boson mixes with an SU(2) septet representation," Phys. Rev. D 87, 053004 (2013) [arXiv:1301.6455 [hep-ph]].

[6] S. Kanemura, M. Kikuchi and K. Yagyu, "Probing exotic Higgs sectors from the precise measurement of Higgs boson couplings," Phys. Rev. D 88, 015020 (2013) [arXiv:1301.7303 [hep-ph]]. 
[7] H. Georgi and M. Machacek, "Doubly Charged Higgs Bosons," Nucl. Phys. B 262, 463 (1985).

[8] M. S. Chanowitz and M. Golden, "Higgs boson triplets with $M(W)=$ $M(Z) \cos \theta_{W}, "$ Phys. Lett. B 165, 105 (1985).

[9] H. E. Logan and V. Rentala, "All the generalized Georgi-Machacek models," Phys. Rev. D 92, no. 7, 075011 (2015) [arXiv:1502.01275 [hep-ph]].

[10] P. Galison, "Large Weak Isospin and the W Mass," Nucl. Phys. B 232, 26 (1984).

[11] R. W. Robinett, "Extended Strongly Interacting Higgs Theories," Phys. Rev. D 32, 1780 (1985).

[12] H. E. Logan, "Radiative corrections to the Z b anti-b vertex and constraints on extended Higgs sectors," hep-ph/9906332.

[13] S. Chang, C. A. Newby, N. Raj and C. Wanotayaroj, "Revisiting Theories with Enhanced Higgs Couplings to Weak Gauge Bosons," Phys. Rev. D 86, 095015 (2012) [arXiv:1207.0493 [hep-ph]].

[14] G. Aad et al. [ATLAS Collaboration], "Search for a Charged Higgs Boson Produced in the Vector-Boson Fusion Mode with Decay $H^{ \pm} \rightarrow W^{ \pm} Z$ using $p p$ Collisions at $\sqrt{s}=8 \mathrm{TeV}$ with the ATLAS Experiment," Phys. Rev. Lett. 114, no. 23, 231801 (2015) [arXiv:1503.04233 [hep-ex]].

[15] A. M. Sirunyan et al. [CMS Collaboration], "Search for Charged Higgs Bosons Produced via Vector Boson Fusion and Decaying into a Pair of $W$ and $Z$ Bosons Using $p p$ Collisions at $\sqrt{s}=13$ TeV," Phys. Rev. Lett. 119, no. 14, 141802 (2017) [arXiv:1705.02942 [hep-ex]]. 
[16] A. M. Sirunyan et al. [CMS Collaboration], "Observation of electroweak production of same-sign $\mathrm{W}$ boson pairs in the two jet and two same-sign lepton final state in proton-proton collisions at $\sqrt{s}=13 \mathrm{TeV}$," Phys. Rev. Lett. 120, no. 8, 081801 (2018) [arXiv:1709.05822 [hep-ex]].

[17] M. Aaboud et al. [ATLAS Collaboration], "Search for resonant $W Z$ production in the fully leptonic final state in proton-proton collisions at $\sqrt{s}=13 \mathrm{TeV}$ with the ATLAS detector," arXiv:1806.01532 [hep-ex].

[18] CMS Collaboration, "Measurement of electroweak WZ production and search for new physics in pp collisions at sqrt(s) $=13 \mathrm{TeV}$," CMS-PAS-SMP-18-001.

[19] M. Aaboud et al. [ATLAS Collaboration], "Search for heavy resonances decaying into $W W$ in the $e \nu \mu \nu$ final state in $p p$ collisions at $\sqrt{s}=13 \mathrm{TeV}$ with the ATLAS detector," Eur. Phys. J. C 78, no. 1, 24 (2018) [arXiv:1710.01123 [hep-ex]].

[20] M. Aaboud et al. [ATLAS Collaboration], "Search for doubly charged scalar bosons decaying into same-sign $W$ boson pairs with the ATLAS detector," arXiv:1808.01899 [hep-ex].

[21] D. de Florian et al. [LHC Higgs Cross Section Working Group], "Handbook of LHC Higgs Cross Sections: 4. Deciphering the Nature of the Higgs Sector," arXiv:1610.07922 [hep-ph].

[22] H. E. Logan and M. B. Reimer, "Characterizing a benchmark scenario for heavy Higgs boson searches in the Georgi-Machacek model," Phys. Rev. D 96, no. 9, 095029 (2017) [arXiv:1709.01883 [hep-ph]].

[23] J. F. Gunion, R. Vega and J. Wudka, "Naturalness problems for rho $=1$ and 
other large one loop effects for a standard model Higgs sector containing triplet fields," Phys. Rev. D 43, 2322 (1991).

[24] S. Blasi, S. De Curtis and K. Yagyu, "Effects of custodial symmetry breaking in the Georgi-Machacek model at high energies," Phys. Rev. D 96, no. 1, 015001 (2017) [arXiv:1704.08512 [hep-ph]].

[25] P. Langacker, "Structure of the standard model," Adv. Ser. Direct. High Energy Phys. 14, 15-36 (1995) [arXiv:hep-ph/0304186 [hep-ph]].

[26] J. Butterworth, "The Standard Model: How far can it go and how can we tell?," Phil. Trans. Roy. Soc. Lond. A 374, no.2075, 20150260 (2016) [arXiv:1601.02759 [hep-ex]].

[27] G. Aad et al. [ATLAS Collaboration], "Observation of a new particle in the search for the Standard Model Higgs boson with the ATLAS detector at the LHC," Phys. Lett. B 716, 1 (2012) [arXiv:1207.7214 [hep-ex]]; S. Chatrchyan et al. [CMS Collaboration], "Observation of a new boson at a mass of $125 \mathrm{GeV}$ with the CMS experiment at the LHC," Phys. Lett. B 716, 30 (2012) [arXiv:1207.7235 [hep-ex]].

[28] R.H. Parker, C. Yu, W. Zhong, B. Estey, and H. Muller "The Standard Model: How far can it go and how can we tell?," Science 360, 191-195 (2018) [arXiv:1812.04130 [physics.atom-ph]].

[29] B. Schulz, "Review on the quantization of gravity," [arXiv:1409.7977 [gr-qc]].

[30] S. Bilenky, "Neutrinos: Majorana or Dirac?," [arXiv:2008.02110 [hep-ph]].

[31] M. Perelstein, "Introduction to Collider Physics," [arXiv:1002.0274 [hep-ph]]. 
[32] G. Apollinari, O. Brüning, T. Nakamoto and L. Rossi, "High Luminosity Large Hadron Collider HL-LHC," [arXiv:1705.08830 [physics.acc-ph]].

[33] D. M. Asner, T. Barklow, C. Calancha, K. Fujii, N. Graf, H. E. Haber, A. Ishikawa, S. Kanemura, S. Kawada and M. Kurata, et al. "ILC Higgs White Paper," [arXiv:1310.0763 [hep-ph]].

[34] A. Blondel, J. Gluza, S. Jadach, P. Janot, T. Riemann, S. Abreu, J. J. AguileraVerdugo, A. B. Arbuzov, J. Baglio and S. D. Bakshi, et al. "Theory for the FCC-ee: Report on the 11th FCC-ee Workshop Theory and Experiments," [arXiv:1905.05078 [hep-ph]].

[35] M.E. Peskin and D.V. Schroeder An Introduction to Quantum Field Theory. Advanced Book Classics. Addison-Wesley Publishing Company, 1995.

[36] T. Cohen, "As Scales Become Separated: Lectures on Effective Field Theory," PoS TASI2018, 011 (2019) [arXiv:1903.03622 [hep-ph]].

[37] E. Noether, "Invariante Variationsprobleme" Nachrichten von der Gesellschaft der Wissenschaften zu Göttingen, Mathematisch-Physikalische Klasse 235-257 (1918).

[38] H. E. Logan, "TASI 2013 lectures on Higgs physics within and beyond the Standard Model," [arXiv:1406.1786 [hep-ph]].

[39] J. Goldstone, A. Salam and S. Weinberg, "Broken Symmetries," Phys. Rev. 127, 965-970 (1962).

[40] S Gasiorowicz, Quantum Physics. Wiley, 2003. 
[41] P. Sikivie, L. Susskind, M. B. Voloshin and V. I. Zakharov, "Isospin Breaking in Technicolor Models," Nucl. Phys. B 173, 189-207 (1980).

[42] A. Freitas, "TASI 2020 Lectures on Precision Tests of the Standard Model," [arXiv:2012.11642 [hep-ph]].

[43] C.G. Callan, "Broken Symmetries," Phys. Rev. D2, 1541 (1970).

[44] K. Symanzik, "Broken Symmetries," Comm. Math. Phys. 18, 227 (1970).

[45] T. P. Cheng, E. Eichten and L. F. Li, "Higgs Phenomena in Asymptotically Free Gauge Theories," Phys. Rev. D 9, 2259 (1974).

[46] F. Lyonnet, I. Schienbein, F. Staub and A. Wingerter, "PyR@TE: Renormalization Group Equations for General Gauge Theories," Comput. Phys. Commun. 185 (2014) 1130 [arXiv:1309.7030 [hep-ph]].

[47] F. Staub, T. Ohl, W. Porod and C. Speckner, "A Tool Box for Implementing Supersymmetric Models," Comput. Phys. Commun. 183 (2012) 2165 [arXiv:1109.5147 [hep-ph]].

[48] G. Buchalla, A. J. Buras and M. E. Lautenbacher, "Weak decays beyond leading logarithms," Rev. Mod. Phys. 68, 1125-1144 (1996) [arXiv:hep-ph/9512380 [hep$\mathrm{ph}]]$.

[49] A. de Gouvea, [arXiv:hep-ph/0411274 [hep-ph]].

[50] S. F. King, "Neutrino mass models," Rept. Prog. Phys. 67, 107-158 (2004) [arXiv:hep-ph/0310204 [hep-ph]].

[51] B. Abi et al. [Muon $g-2$ Collaboration], "Measurement of the Positive Muon Anomalous Magnetic Moment to 0.46 ppm” Phys. Rev. Lett. 126, 141801 (2021). 
[52] R. Aaij et al. [LHCb], "Test of lepton universality in beauty-quark decays," [arXiv:2103.11769 [hep-ex]].

[53] E. Corbelli and P. Salucci, "The Extended Rotation Curve and the Dark Matter Halo of M33," Mon. Not. Roy. Astron. Soc. 311, 441-447 (2000) [arXiv:astroph/9909252 [astro-ph]].

[54] B. Famaey and S. McGaugh, "Modified Newtonian Dynamics (MOND): Observational Phenomenology and Relativistic Extensions," Living Rev. Rel. 15, 10 (2012) [arXiv:1112.3960 [astro-ph.CO]].

[55] D. Clowe, A. Gonzalez and M. Markevitch, "Weak lensing mass reconstruction of the interacting cluster 1E0657-558: Direct evidence for the existence of dark matter," Astrophys. J. 604, 596-603 (2004) [arXiv:astro-ph/0312273 [astro-ph]].

[56] N. Aghanim et al. [Planck], "Planck 2018 results. VI. Cosmological parameters," Astron. Astrophys. 641, A6 (2020) [arXiv:1807.06209 [astro-ph.CO]].

[57] J. A. Vázquez, L. E. Padilla and T. Matos, "Inflationary Cosmology: From Theory to Observations," [arXiv:1810.09934 [astro-ph.CO]].

[58] J. M. Cline, "Baryogenesis," [arXiv:hep-ph/0609145 [hep-ph]].

[59] S. Davidson, E. Nardi and Y. Nir, "Leptogenesis," Phys. Rept. 466, 105-177 (2008) [arXiv:0802.2962 [hep-ph]].

[60] W. Skiba, "Effective Field Theory and Precision Electroweak Measurements," [arXiv:1006.2142 [hep-ph]].

[61] S. P. Martin, "A Supersymmetry primer," Adv. Ser. Direct. High Energy Phys. 18, 1-98 (1998) [arXiv:hep-ph/9709356 [hep-ph]]. 
[62] F. Goertz, "Composite Higgs theory," PoS ALPS2018, 012 (2018) [arXiv:1812.07362 [hep-ph]].

[63] M. Schmaltz and D. Tucker-Smith, "Little Higgs review," Ann. Rev. Nucl. Part. Sci. 55, 229-270 (2005) [arXiv:hep-ph/0502182 [hep-ph]].

[64] R. Barbieri, L. J. Hall and K. Harigaya, "Minimal Mirror Twin Higgs," JHEP 11, 172 (2016) [arXiv:1609.05589 [hep-ph]].

[65] N. Arkani-Hamed, T. Cohen, R. T. D’Agnolo, A. Hook, H. D. Kim and D. Pinner, "Solving the Hierarchy Problem at Reheating with a Large Number of Degrees of Freedom," Phys. Rev. Lett. 117, no.25, 251801 (2016) [arXiv:1607.06821 [hepph]].

[66] R. M. Schabinger and J. D. Wells, "A Minimal spontaneously broken hidden sector and its impact on Higgs boson physics at the large hadron collider," Phys. Rev. D 72, 093007 (2005) [arXiv:hep-ph/0509209 [hep-ph]].

[67] B. Patt and F. Wilczek, "Higgs-field portal into hidden sectors," [arXiv:hepph/0605188 [hep-ph]].

[68] M. Bowen, Y. Cui and J. D. Wells, "Narrow trans-TeV Higgs bosons and H $\longrightarrow$ hh decays: Two LHC search paths for a hidden sector Higgs boson," JHEP 03, 036 (2007) [arXiv:hep-ph/0701035 [hep-ph]].

[69] G. C. Branco, P. M. Ferreira, L. Lavoura, M. N. Rebelo, M. Sher and J. P. Silva, "Theory and phenomenology of two-Higgs-doublet models," Phys. Rept. 516, 1-102 (2012) [arXiv:1106.0034 [hep-ph]].

[70] P. Fileviez Perez, T. Han, G. y. Huang, T. Li and K. Wang, "Neutrino Masses 
and the CERN LHC: Testing Type II Seesaw," Phys. Rev. D 78, 015018 (2008) [arXiv:0805.3536 [hep-ph]].

[71] B. W. Lee, C. Quigg and H. B. Thacker, "The Strength of Weak Interactions at Very High-Energies and the Higgs Boson Mass," Phys. Rev. Lett. 38, 883-885 (1977).

[72] B. W. Lee, C. Quigg and H. B. Thacker, "Weak Interactions at Very HighEnergies: The Role of the Higgs Boson Mass," Phys. Rev. D 16, 1519 (1977).

[73] K. Hally, H. E. Logan and T. Pilkington, "Constraints on large scalar multiplets from perturbative unitarity," Phys. Rev. D 85, 095017 (2012) [arXiv:1202.5073 [hep-ph]].

[74] K. Hartling, K. Kumar and H. E. Logan, "The decoupling limit in the GeorgiMachacek model," Phys. Rev. D 90, 015007 (2014) [arXiv:1404.2640 [hep-ph]].

[75] M. Aoki and S. Kanemura, "Unitarity bounds in the Higgs model including triplet fields with custodial symmetry," Phys. Rev. D 77, no. 9, 095009 (2008) [Erratum: Phys. Rev. D 89, no. 5, 059902 (2014)] [arXiv:0712.4053 [hep-ph]].

[76] K. Hartling, K. Kumar and H. E. Logan, "GMCALC: a calculator for the GeorgiMachacek model," arXiv:1412.7387 [hep-ph].

[77] A. M. Sirunyan et al. [CMS], "Search for charged Higgs bosons produced in vector boson fusion processes and decaying into vector boson pairs in proton-proton collisions at $\sqrt{s}=13 \mathrm{TeV}, "$ Eur. Phys. J. C 81, no.8, 723 (2021) [arXiv:2104.04762 [hep-ex]]. 
[78] K. Hartling, K. Kumar and H. E. Logan, "Indirect constraints on the GeorgiMachacek model and implications for Higgs boson couplings," Phys. Rev. D 91, no.1, 015013 (2015) [arXiv:1410.5538 [hep-ph]].

[79] P. Bechtle, O. Brein, S. Heinemeyer, G. Weiglein and K. E. Williams, "HiggsBounds: Confronting Arbitrary Higgs Sectors with Exclusion Bounds from LEP and the Tevatron," Comput. Phys. Commun. 181, 138 (2010) [arXiv:0811.4169 [hep-ph]]; "HiggsBounds 2.0.0: Confronting Neutral and Charged Higgs Sector Predictions with Exclusion Bounds from LEP and the Tevatron," Comput. Phys. Commun. 182, 2605 (2011) [arXiv:1102.1898 [hep-ph]]; P. Bechtle, O. Brein, S. Heinemeyer, O. Stal, T. Stefaniak, G. Weiglein and K. Williams, "Recent Developments in HiggsBounds and a Preview of HiggsSignals," PoS CHARGED 2012, 024 (2012) [arXiv:1301.2345 [hep-ph]]; "HiggsBounds - 4: Improved Tests of Extended Higgs Sectors against Exclusion Bounds from LEP, the Tevatron and the LHC," Eur. Phys. J. C 74, no. 3, 2693 (2014) [arXiv:1311.0055 [hep-ph]]; P. Bechtle, S. Heinemeyer, O. Stal, T. Stefaniak and G. Weiglein, "Applying Exclusion Likelihoods from LHC Searches to Extended Higgs Sectors," Eur. Phys. J. C 75, no. 9, 421 (2015) [arXiv:1507.06706 [hep-ph]].

[80] A. Ismail, H. E. Logan and Y. Wu, "Updated constraints on the Georgi-Machacek model from LHC Run 2," arXiv:2003.02272 [hep-ph].

[81] C. W. Chiang, A. L. Kuo and T. Yamada, "Searches of exotic Higgs bosons in general mass spectra of the Georgi-Machacek model at the LHC," JHEP 01, 120 (2016) [arXiv:1511.00865 [hep-ph]].

[82] G. Aad et al. [ATLAS Collaboration], "Evidence for Electroweak Production of 
$W^{ \pm} W^{ \pm} j j$ in $p p$ Collisions at $\sqrt{s}=8 \mathrm{TeV}$ with the ATLAS Detector," Phys. Rev. Lett. 113, no. 14, 141803 (2014) [arXiv:1405.6241 [hep-ex]].

[83] C. W. Chiang, S. Kanemura and K. Yagyu, "Novel constraint on the parameter space of the Georgi-Machacek model with current LHC data," Phys. Rev. D 90, no. 11, 115025 (2014) [arXiv:1407.5053 [hep-ph]].

[84] G. Aad et al. [ATLAS Collaboration], "Search for anomalous production of prompt same-sign lepton pairs and pair-produced doubly charged Higgs bosons with $\sqrt{s}=8 \mathrm{TeV} p p$ collisions using the ATLAS detector," JHEP 1503, 041 (2015) [arXiv:1412.0237 [hep-ex]].

[85] S. Kanemura, M. Kikuchi, H. Yokoya and K. Yagyu, "LHC Run-I constraint on the mass of doubly charged Higgs bosons in the same-sign diboson decay scenario," PTEP 2015, 051B02 (2015) [arXiv:1412.7603 [hep-ph]].

[86] G. Aad et al. [ATLAS Collaboration], "Search for Scalar Diphoton Resonances in the Mass Range $65-600 \mathrm{GeV}$ with the ATLAS Detector in pp Collision Data at $\sqrt{s}=8 \mathrm{TeV}$," Phys. Rev. Lett. 113, no. 17, 171801 (2014) [arXiv:1407.6583 [hep-ex]].

[87] G. Aad et al. [ATLAS], "Search for doubly and singly charged Higgs bosons decaying into vector bosons in multi-lepton final states with the ATLAS detector using proton-proton collisions at $\sqrt{s}=13 \mathrm{TeV}$," JHEP 06, 146 (2021) [arXiv:2101.11961 [hep-ex]].

[88] M. Aaboud et al. [ATLAS Collaboration], "Search for doubly charged Higgs boson production in multi-lepton final states with the ATLAS detector using 
proton-proton collisions at $\sqrt{s}=13 \mathrm{TeV}, "$ Eur. Phys. J. C 78, no. 3, 199 (2018) [arXiv:1710.09748 [hep-ex]].

[89] C. W. Chiang, A. L. Kuo and K. Yagyu, "Radiative corrections to Higgs couplings with weak gauge bosons in custodial multi-Higgs models," Phys. Lett. B 774, 119-122 (2017) [arXiv:1707.04176 [hep-ph]].

[90] C. W. Chiang, A. L. Kuo and K. Yagyu, "One-loop renormalized Higgs boson vertices in the Georgi-Machacek model," Phys. Rev. D 98, no.1, 013008 (2018) [arXiv:1804.02633 [hep-ph]].

[91] J. Alwall et al., "The automated computation of tree-level and next-to-leading order differential cross sections, and their matching to parton shower simulations," JHEP 1407, 079 (2014) [arXiv:1405.0301 [hep-ph]].

[92] J. Butterworth et al., "PDF4LHC recommendations for LHC Run II," J. Phys. G 43, 023001 (2016) [arXiv:1510.03865 [hep-ph]].

[93] M. Zaro and H. Logan, "Recommendations for the interpretation of LHC searches for $H_{5}^{0}, H_{5}^{ \pm}$, and $H_{5}^{ \pm \pm}$in vector boson fusion with decays to vector boson pairs," LHCHXSWG-2015-001.

[94] C. Degrande, K. Hartling and H. E. Logan, "Scalar decays to $\gamma \gamma, Z \gamma$, and $W \gamma$ in the Georgi-Machacek model," Phys. Rev. D 96, no.7, 075013 (2017) [erratum: Phys. Rev. D 98, no.1, 019901 (2018)] [arXiv:1708.08753 [hep-ph]].

[95] M. E. Peskin and T. Takeuchi, "A New constraint on a strongly interacting Higgs sector," Phys. Rev. Lett. 65, 964 (1990); "Estimation of oblique electroweak corrections," Phys. Rev. D 46, 381 (1992). 
[96] S. Chang, "A 'Littlest Higgs' model with custodial SU(2) symmetry," JHEP 0312, 057 (2003) [hep-ph/0306034].

[97] S. Chang and J. G. Wacker, "Little Higgs and custodial SU(2)," Phys. Rev. D 69, 035002 (2004) [hep-ph/0303001].

[98] C. Patrignani et al. [Particle Data Group], "Review of Particle Physics," Chin. Phys. C 40, no. 10, 100001 (2016).

[99] G. Aad et al. [ATLAS], "Combined measurements of Higgs boson production and decay using up to $80 \mathrm{fb}^{-1}$ of proton-proton collision data at $\sqrt{s}=13 \mathrm{TeV}$ collected with the ATLAS experiment," Phys. Rev. D 101, no.1, 012002 (2020) [arXiv:1909.02845 [hep-ex]].

[100] S. Dawson et al., "Working Group Report: Higgs Boson," [arXiv:1310.8361 [hep-ex]].

[101] D. d'Enterria, "Physics case of FCC-ee," Frascati Phys. Ser. 61, 17 (2016) [arXiv:1601.06640 [hep-ex]].

[102] Y. Chen, J. Lykken, M. Spiropulu, D. Stolarski and R. Vega-Morales, "Golden Probe of Electroweak Symmetry Breaking," Phys. Rev. Lett. 117, no. 24, 241801 (2016) [arXiv:1608.02159 [hep-ph]].

[103] A. Delgado, M. Garcia-Pepin, M. Quiros, J. Santiago and R. Vega-Morales, "Diphoton and Diboson Probes of Fermiophobic Higgs Bosons at the LHC," JHEP 1606, 042 (2016) [arXiv:1603.00962 [hep-ph]].

[104] H. E. Logan and Y. Wu, "Searching for the $W \gamma$ decay of a charged Higgs boson," JHEP 1811, 121 (2018) [arXiv:1809.09127 [hep-ph]]. 
[105] C.-W. Chiang and K. Yagyu, "Testing the custodial symmetry in the Higgs sector of the Georgi-Machacek model," JHEP 1301, 026 (2013) [arXiv:1211.2658 [hep-ph]].

[106] C. W. Chiang, S. Kanemura and K. Yagyu, "Phenomenology of the GeorgiMachacek model at future electron-positron colliders," Phys. Rev. D 93, no. 5, 055002 (2016) [arXiv:1510.06297 [hep-ph]].

[107] B. P. Jaeger et al., "In situ measurements of the ATLAS jet energy resolution using 13 TeV pp data," ATL-PHYS-SLIDE-2019-053.

[108] T. Behnke et al., "The International Linear Collider Technical Design Report Volume 4: Detectors," arXiv:1306.6329 [physics.ins-det].

[109] Y. Hamada, K. Kawana and K. Tsumura, "Landau pole in the Standard Model with weakly interacting scalar fields," Phys. Lett. B 747, 238 (2015) [arXiv:1505.01721 [hep-ph]].

[110] A. Goudelis, B. Herrmann and O. Stal, "Dark matter in the Inert Doublet Model after the discovery of a Higgs-like boson at the LHC," JHEP 1309, 106 (2013) [arXiv:1303.3010 [hep-ph]]. 